\title{
Liquid Chromatography -- Tandem Mass Spectrometry Techniques for Drugs of Abuse as Applied to Bacterial and Forensic Toxicology
}

Stephanie Marie Martindale

West Virginia University

Follow this and additional works at: https://researchrepository.wvu.edu/etd

\section{Recommended Citation \\ Martindale, Stephanie Marie, "Liquid Chromatography -- Tandem Mass Spectrometry Techniques for Drugs of Abuse as Applied to Bacterial and Forensic Toxicology" (2013). Graduate Theses, Dissertations, and Problem Reports. 305.}

https://researchrepository.wvu.edu/etd/305

This Dissertation is protected by copyright and/or related rights. It has been brought to you by the The Research Repository @ WVU with permission from the rights-holder(s). You are free to use this Dissertation in any way that is permitted by the copyright and related rights legislation that applies to your use. For other uses you must obtain permission from the rights-holder(s) directly, unless additional rights are indicated by a Creative Commons license in the record and/ or on the work itself. This Dissertation has been accepted for inclusion in WVU Graduate Theses, Dissertations, and Problem Reports collection by an authorized administrator of The Research Repository @ WVU.

For more information, please contact researchrepository@mail.wvu.edu. 
Liquid Chromatography - Tandem Mass Spectrometry Techniques for Drugs of Abuse as Applied to Bacterial and Forensic Toxicology

Stephanie Marie Martindale

\author{
Dissertation submitted \\ to the Eberly College of Arts and Sciences \\ at West Virginia University \\ Doctor of Philosophy in \\ Chemistry \\ Suzanne Bell, Ph.D., Chair \\ Harry Finklea, Ph.D. \\ Glen Jackson, Ph.D. \\ Stephen Valentine, Ph.D. \\ Gary Bissonnette, Ph.D. \\ Department of Chemistry
}

in partial fulfillment of the requirements for the degree of

\author{
Morgantown, West Virginia \\ 2013
}

Keywords: LC-MS/MS; Reinforced Clostridial Medium; Anaerobic Bacteria;

Diazepam; Flunitrazepam; Cocaine; Fentanyl; Forensic Toxicology

Copyright $\odot 2013$ Stephanie Marie Martindale 


\title{
ABSTRACT
}

\section{Liquid Chromatography - Tandem Mass Spectrometry Techniques for Drugs of Abuse as Applied to Bacterial and Forensic Toxicology}

\author{
Stephanie Marie Martindale
}

The ability of bacterial species to transform complex chemical substances has been well documented. Some of these species are native to the human gastrointestinal tract and play an active role in the postmortem decomposition process. These species have potential to cause biotransformations that affect compound-to-metabolite ratios within the human body, especially after death. Postmortem changes such as these have rarely been evaluated or taken into consideration but they have potential to supply valuable information, especially concerning compound identification and confirmation.

The purpose of this research was to investigate the effects of Escherichia coli, Bacteroides fragilis, and Clostridium perfringens on drugs of abuse, and to compare these metabolites to those produced during normal human metabolism. To analyze and quantify these effects, a novel liquid chromatography triple quadrupole tandem mass spectrometry method was developed for diazepam, flunitrazepam, and metabolites in Reinforced Clostridial Medium. Reinforced Clostridial Medium is a complex matrix designed to provide the nutrients necessary to promote growth of bacterial species, particularly Clostridia, other anaerobes, and specimens in clinical samples. To date, similar methods of extraction, quantitation, and analysis of drugs of abuse in a nutrient medium matrix have not been published.

After validation, the method was applied to assess the specific effects of human gastrointestinal species on the targeted drugs of abuse. Experiments explored the effects of individual bacterial species as well as a mixed culture on the benzodiazepine compounds under anaerobic conditions. Diazepam and flunitrazepam were selected for biotransformation studies because of their potential for abuse and prevalence in analysis of both clinical and forensic specimens. Additionally, the human metabolites for these compounds had been previously identified and reference standards were commercially available.

A series of bacterial blanks were prepared in Reinforced Clostridial Medium and incubated under experimental conditions. None of the bacterial specimens produced interferences with the target analytes that satisfied peak shape, retention time, ion ratio, and limit of quantitation requirements.

Analyte blanks were also prepared for analysis. Investigation of analyte blanks and the mass balance for the reaction suggested that diazepam performed a partial, unassisted degradation when incubated under experimental conditions. However, neither nordiazepam, oxazepam, nor temazepam registered an increase between preto post-incubation concentrations. The degradation product was therefore unable to be identified under current experimental conditions. In biotransformation studies, 
diazepam showed a decrease in concentration between pre- and post-incubation for the Escherichia coli and Bacteroides fragilis series of samples, while changes were minimal for Clostridium perfringens and the mixed culture. Findings suggested that Escherichia coli and Bacteroides fragilis were acting on diazepam; however there was no increase in concentration for the human metabolites monitored. The discrepancy in mass balance was potentially indicative of a unique bacterial metabolite not produced during normal human metabolism.

Analysis of the flunitrazepam drug blanks likewise exhibited a decrease in concentration. Unlike what was observed in the diazepam blank experiments however, there was a corresponding increase in concentration for the 7-aminoflunitrazepam metabolite in post-incubation samples. This accounted for some of the flunitrazepam conversion. In biotransformation studies, samples incubated with Escherichia coli demonstrated a greater decrease in flunitrazepam concentration than what was seen in analyte blank samples, while Bacteroides fragilis, Clostridium perfringens, and the mixed culture resulted in nearly complete conversion of flunitrazepam. Increased 7aminoflunitrazepam concentrations accounted for the majority of the conversion; however data suggested production of a minor metabolite that was not monitored in the current analysis.

These experiments served as a pilot study and proof of concept. Unlike previous research, animal models were not required for experimental purposes, which eliminated the ethical considerations and guidelines required for animal research. Frozen stock cultures of bacterial samples provided a theoretically endless supply of specimens for transformation studies, reducing cost. The streamlined methodology allowed for quantities of experimental variations to be performed in unison, conserving time. Extraction procedures, a narrow bore column, and low flow rate for instrumental analysis minimized solvent consumption and hazardous waste production, making analysis both cost-effective and environmentally friendly.

The presented experimental methodology serves as a template and can be adapted and applied to a realm of possibilities including investigation of natural products, biodegradation of agricultural and environmental contaminants, isolation and repurpose of native bacterial enzymes, as well as further studies on pharmaceutical compounds and drugs of abuse. An additional method was developed and validated for quantitation of cocaine, fentanyl, and metabolites in Reinforced Clostridial Medium, which will be applied in similar fashion. The applications can easily be expanded to include alternative bacterial species as well. Ultimately, this methodology would be ideal to study compounds that are too toxic or lethal for animal and human metabolic investigations. This would be particularly useful in military explorations of exposure to incapacitation and chemical warfare agents. 


\section{Dedication}

For my parents Rick Martindale and Dennis and Patricia Azlin and my sister Alexandria Azlin who serve as constant sources of encouragement, support, and love.

For my grandparents. LaVaughn Hale for the cheers, love, and sharing this accomplishment with me. Charles Azlin, Gene Hale, and Frank and Lorraine Schram for being my guardian angels and helping me complete my journey.

And to Steve Keschinger and Lonestar Magoo who make life fun and worth living. 


\section{Acknowledgments}

I would like to express gratitude and acknowledge the following individuals, groups, and facilities.

The past and current members of the Bell Research Group in the Chemistry Department at West Virginia University for their advice, support, and friendship.

Dr. Lucy Oldfield for being my co-advisor, cheerleader, and friend.

Dr. Elizabeth Yates, Jim Arndt, Lee Greenawald, Corey Nida, and Liz Ward for proofreading, support, and friendship.

Melissa Dixon for her knowledge of microbiology, support, and friendship.

The United States Army Edgewood Chemical Biological Center (ECBC) for unwavering guidance and support.

Dr. Frederic Berg for taking me in and allowing me to use the instrumentation and facilities at ECBC. And for helping me keep a sense of humor.

Dr. Sue Bae for her knowledge and guidance.

Dr. Margaret MacDonald and Vikki Henderson for allowing me to set up my anaerobic chamber in their lab space and being so accommodating.

Dennis Bevilacqua, Dr. Mike Ellzy, Dr. Bruce King, and Ken Sumpter for being the fairy godparents of ECBC and ALWAYS lending a helping hand.

Dr. Steve Harvey for helping with bacterial methodology and lending me the anaerobic chamber.

Nicole Rippeon and Jen Stinebert for helping me navigate the red tape, allowing me to vent, and making me laugh at the ridiculousness we encountered.

Nancy Skrla for encouraging my love of chemistry.

ECBC - United States Army Medical Research Institute of Chemical Defense (USAMRICD) Proteomics Core Facility for use of the facility and instrumentation.

The Department of Defense Science, Mathematics \& Research for Transformation (SMART Program) for the fellowship and opportunity.

Dr. Robert Powers for his creativity and knowledge.

My committee members, Dr. Suzanne Bell, Dr. Harry Finklea, Dr. Glen Jackson, Dr. Stephen Valentine, and Dr. Gary Bissonnette for reviewing my document as well as serving on my graduate committee. 


\section{Table of Contents}

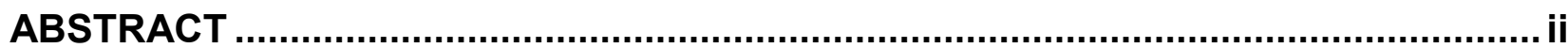

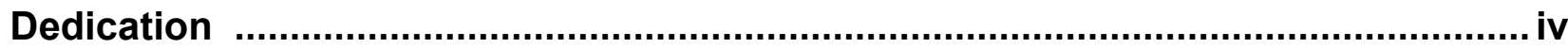

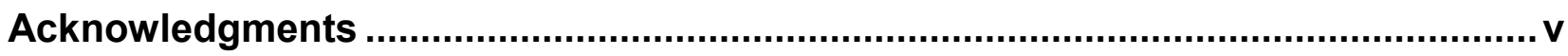

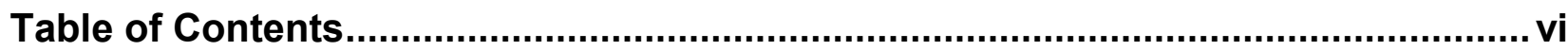

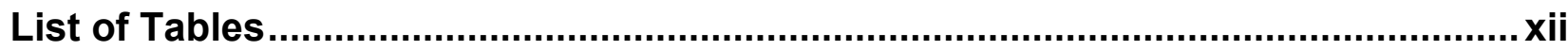

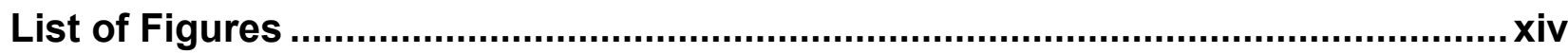

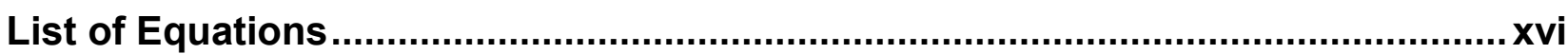

List of Abbreviations, Symbols and Nomenclature ................................................

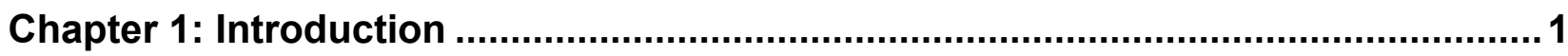

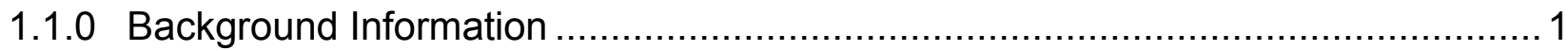

1.1.1 Agricultural Applications and Environmental Contaminants ….................... 2

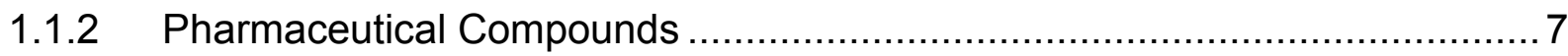

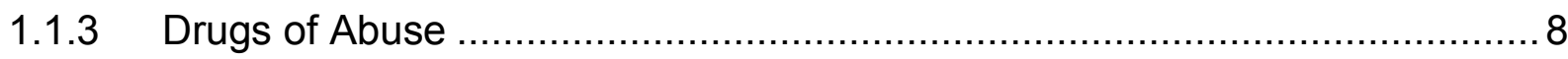

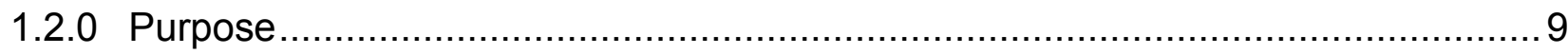

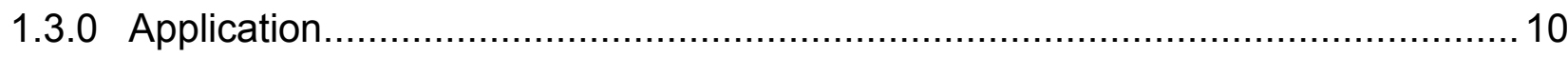

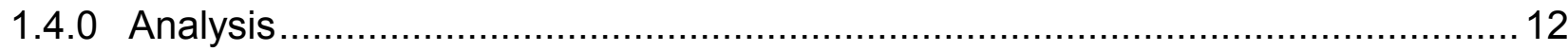

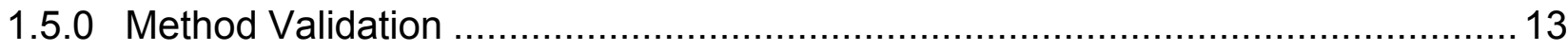

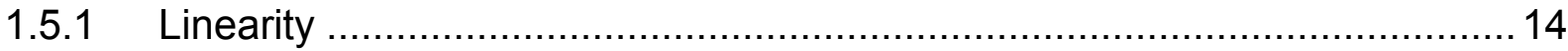

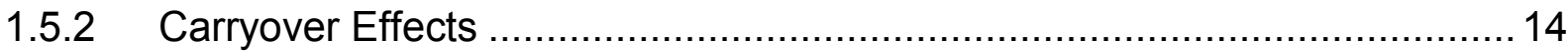

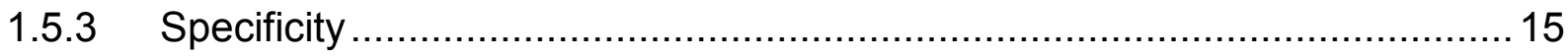

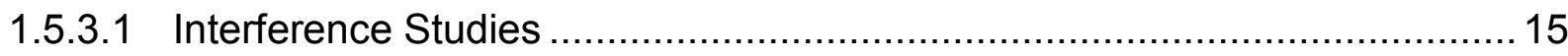

1.5.3.2 Ionization Suppression/Enhancement and Recovery ............................ 15

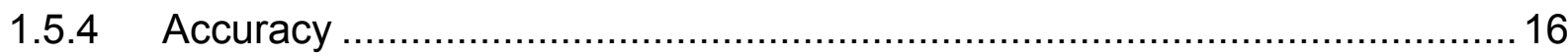




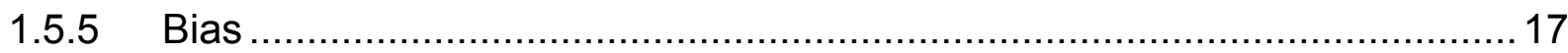

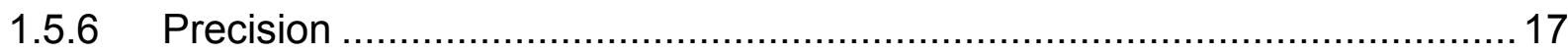

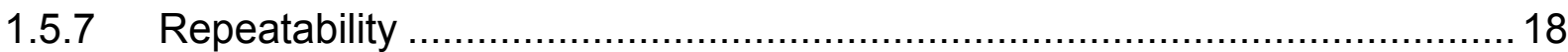

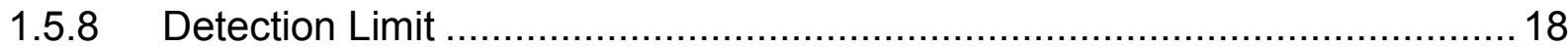

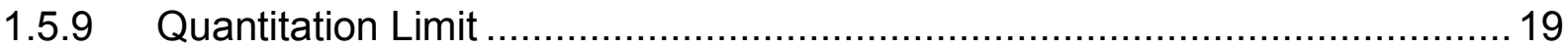

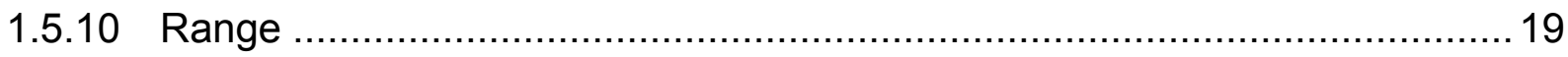

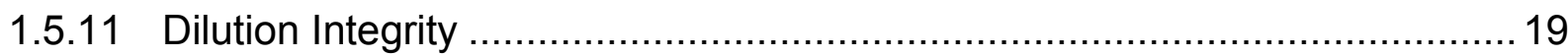

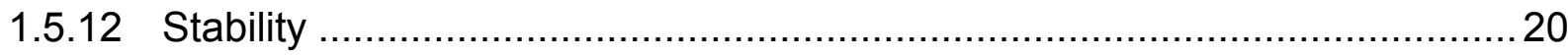

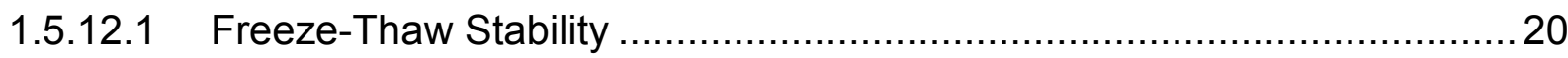

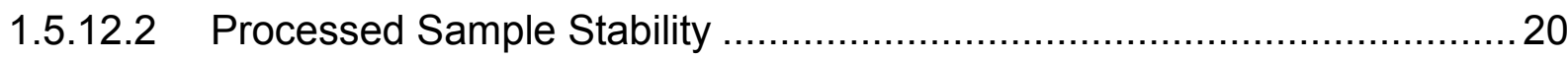

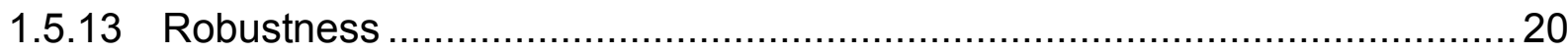

Chapter 2: Optimization of Bacterial Growth and Preparation .................................21

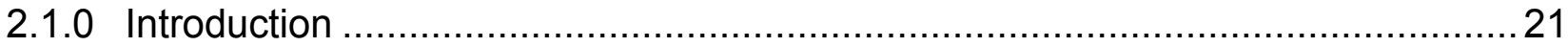

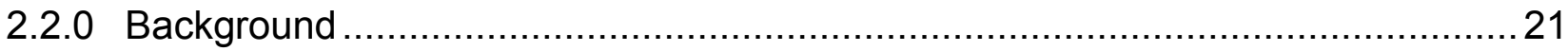

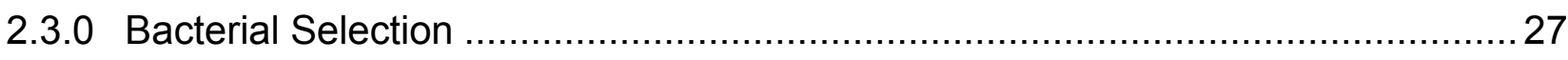

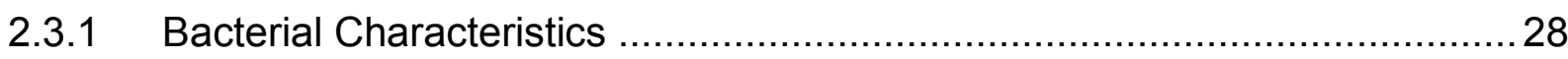

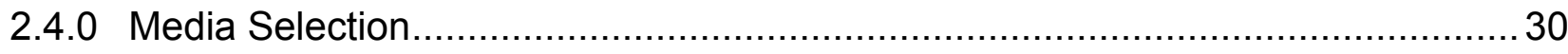

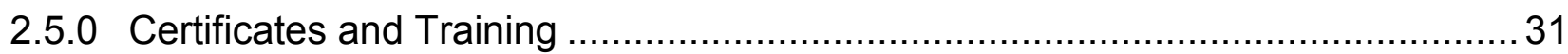

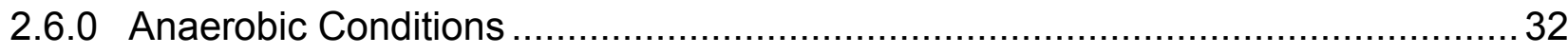

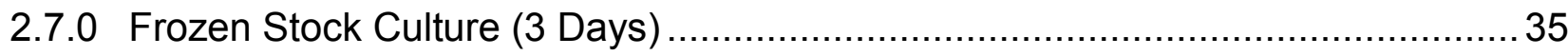

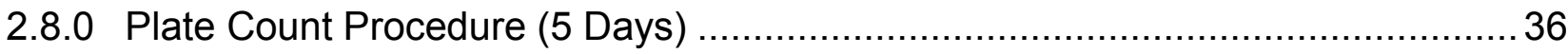

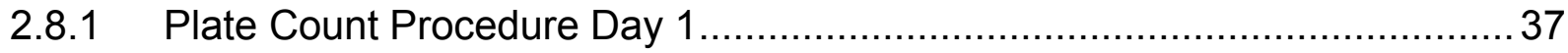

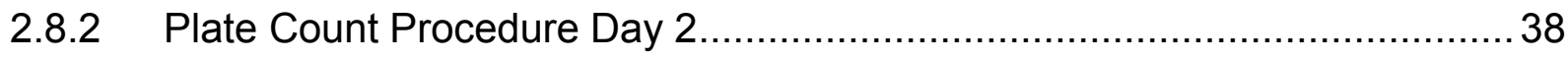

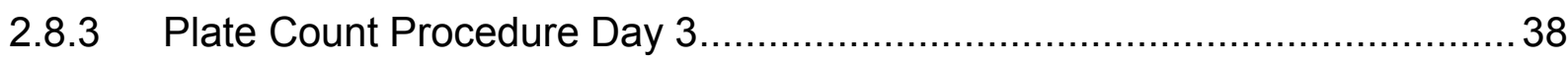

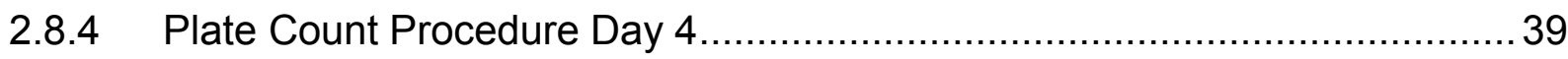


2.8.5 Plate Count Procedure Day 5 .............................................................. 42

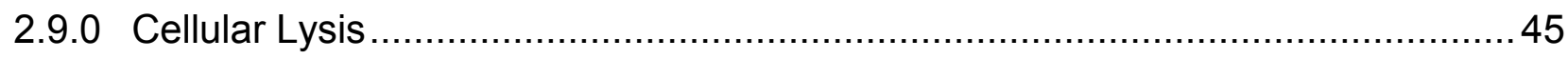

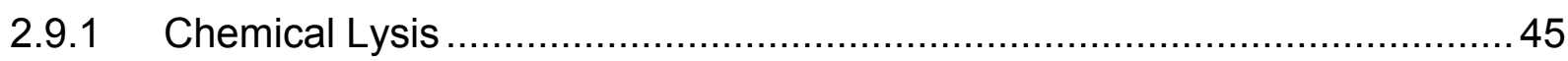

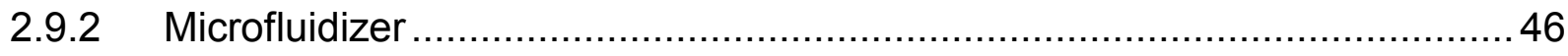

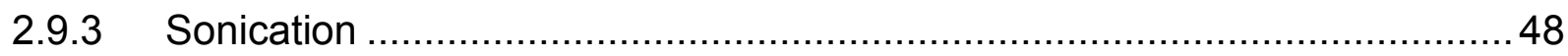

Chapter 3: Method Validation - Simultaneous Quantitation of Diazepam, Flunitrazepam, and Metabolites in Reinforced Clostridial Medium by Liquid Chromatography-Tandem Mass Spectrometry...........................49

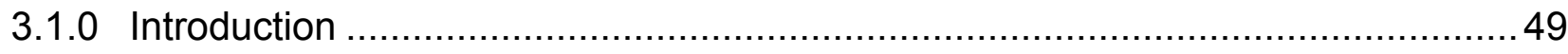

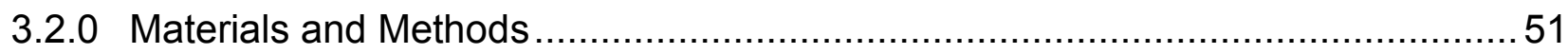

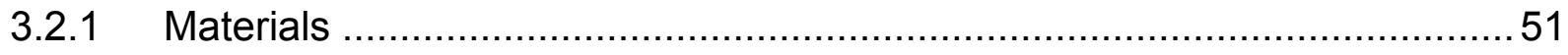

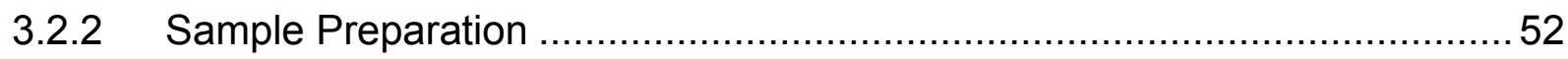

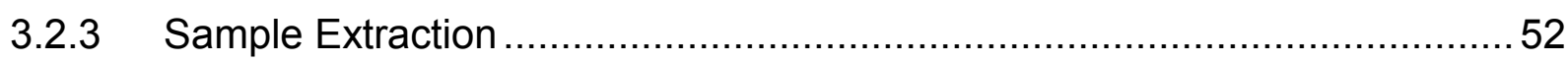

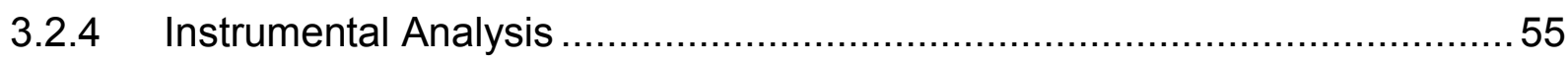

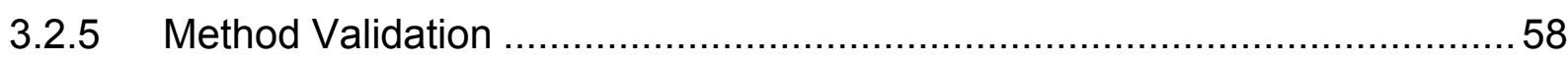

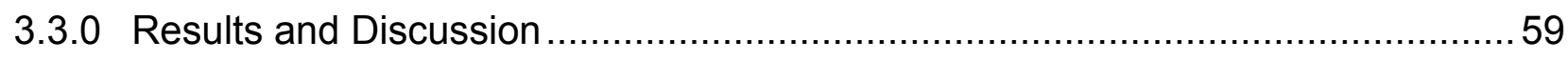

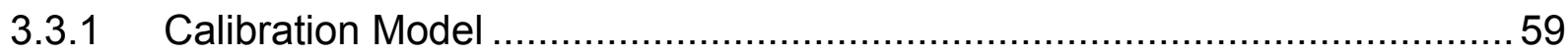

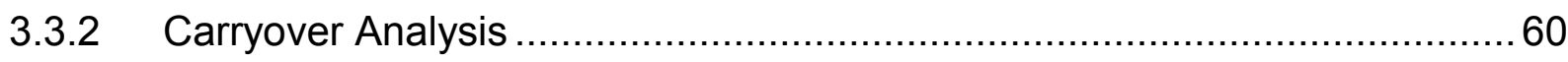

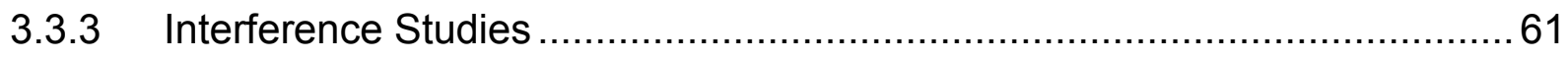

3.3.4 Ionization Suppression/Enhancement and Recovery ..............................61

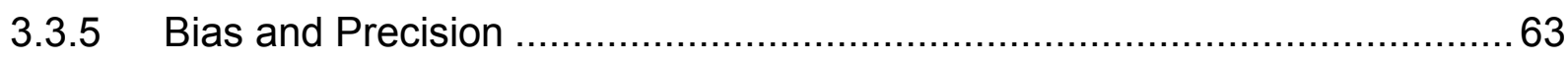

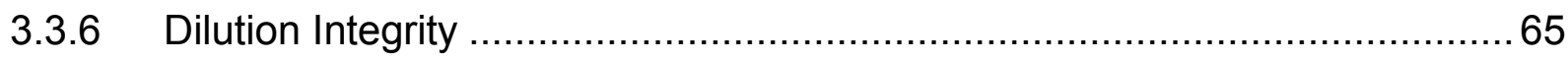

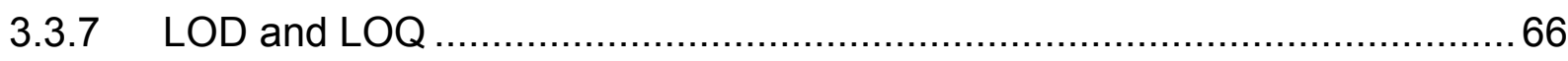

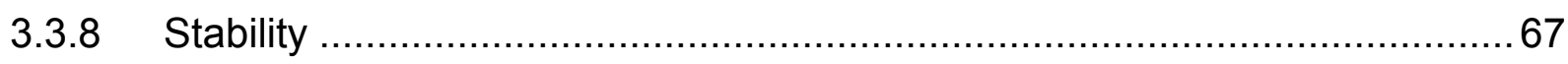

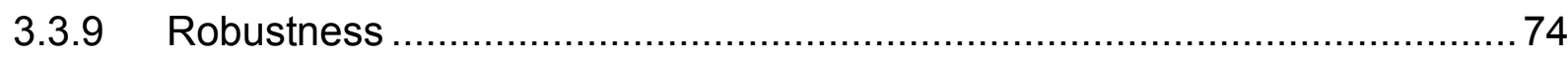

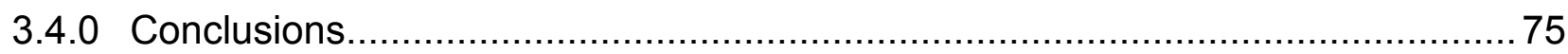




\section{Chapter 4: Detection and Quantitation of Diazepam and Flunitrazepam}

Metabolites Produced by Anaerobic Bacterial Species in Reinforced Clostridial Medium using Liquid Chromatography-Tandem Mass

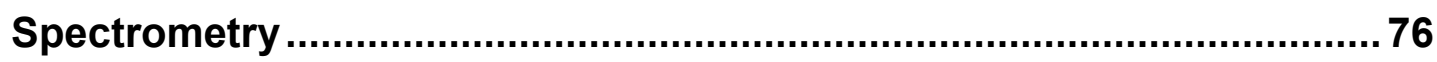

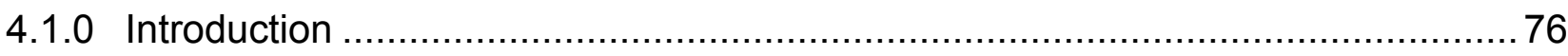

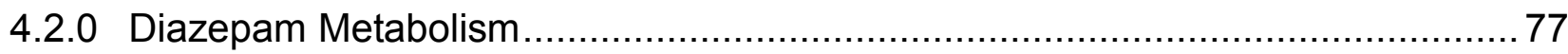

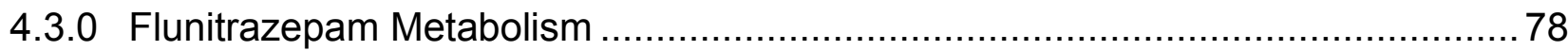

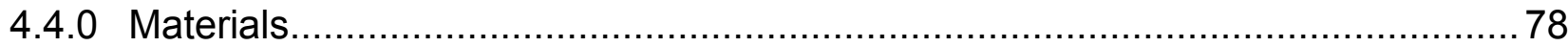

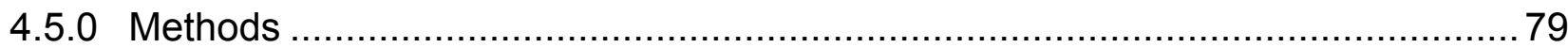

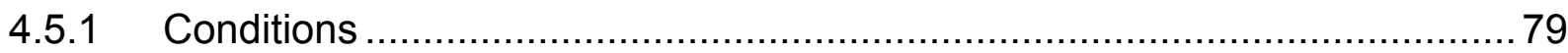

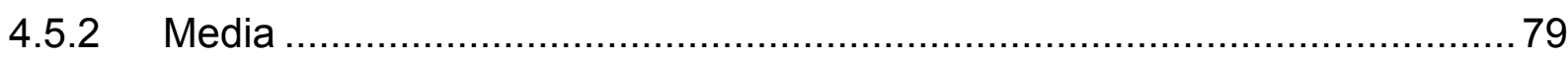

4.5.3 Bacterial Sample Preparation........................................................... 79

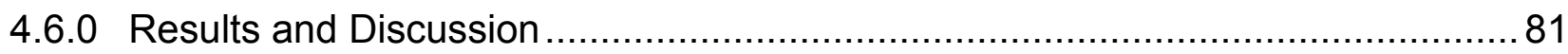

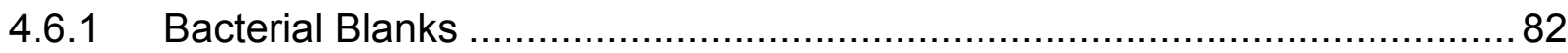

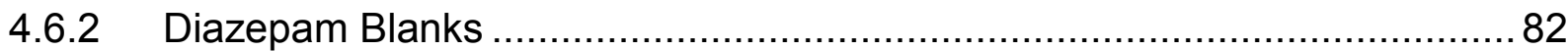

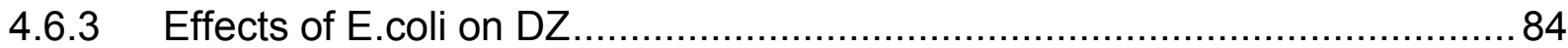

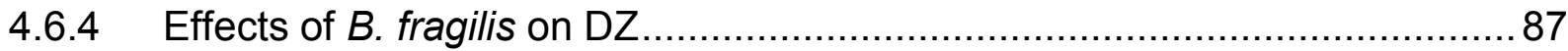

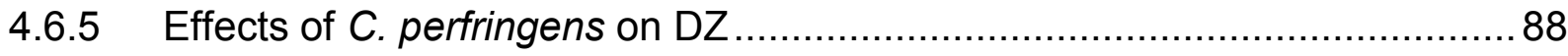

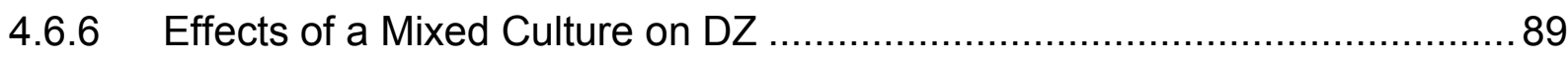

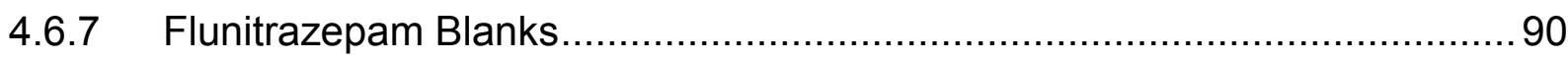

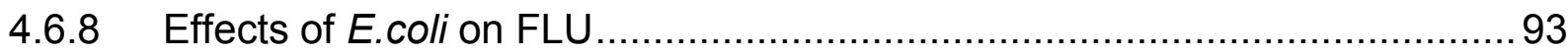

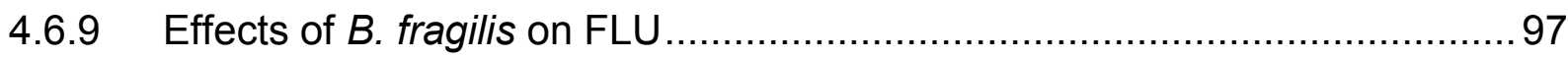

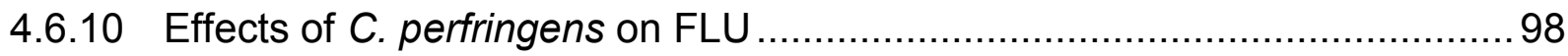

4.6.11 Effects of a Mixed Culture on FLU …................................................ 100

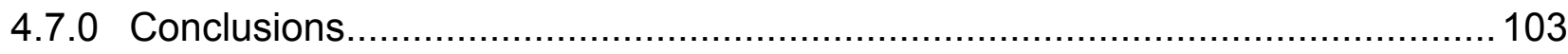


Chapter 5 - A Validated Method for the Quantitation of Cocaine, Fentanyl, and Metabolites in Reinforced Clostridial Medium by Liquid Chromatography-Tandem Mass Spectrometry ....................................106

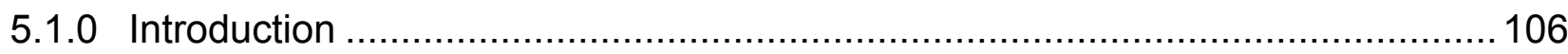

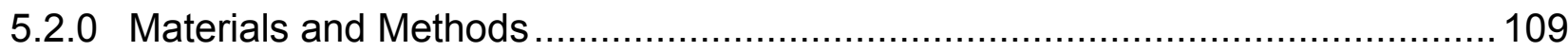

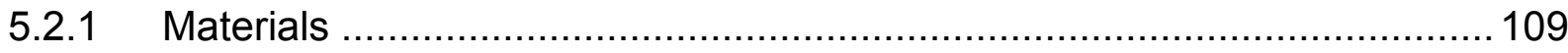

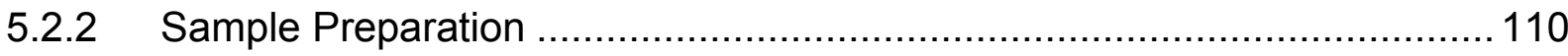

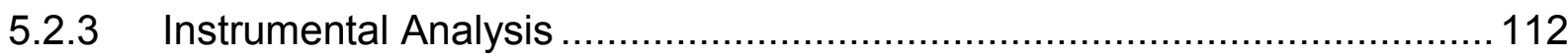

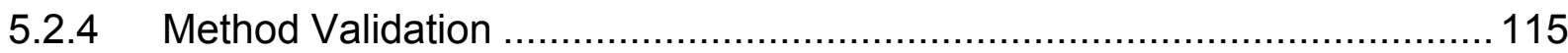

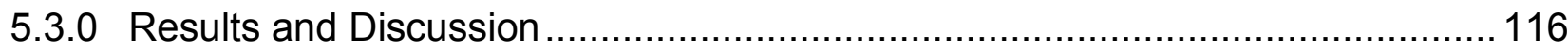

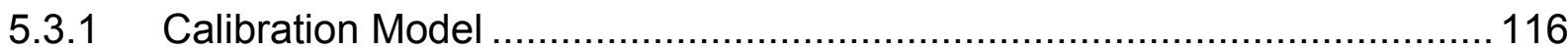

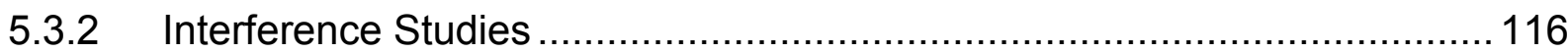

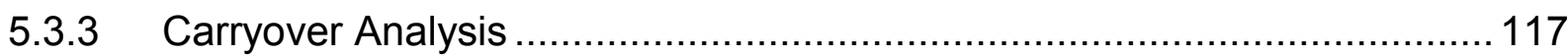

5.3.4 Ionization Suppression/Enhancement and Recovery ........................... 117

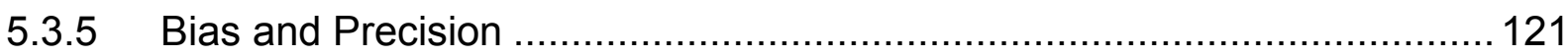

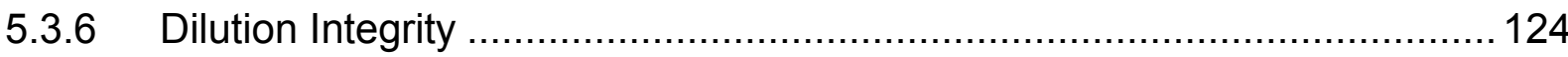

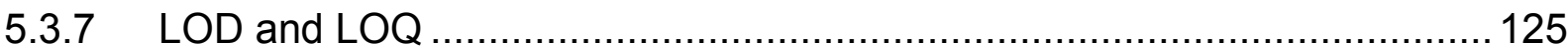

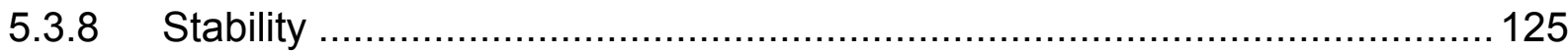

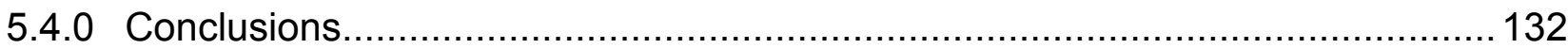

Chapter 6: Research Summary, Implications, and Future Works.......................... 133

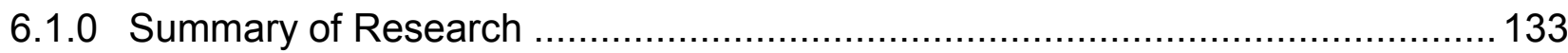

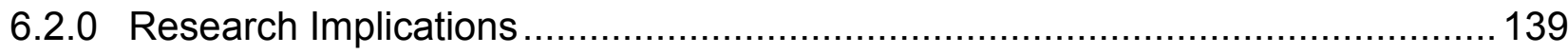

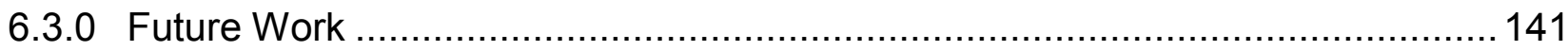

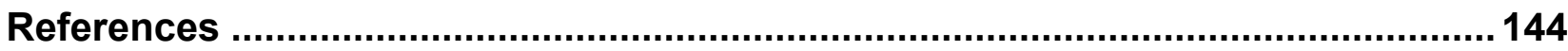

Appendix A - Calibration Curves for Diazepam, Flunitrazepam, and Metabolites in

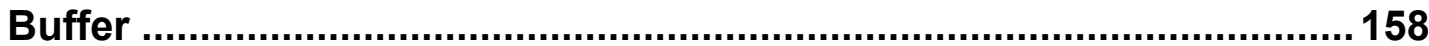


Appendix B - Calibration Curves for Diazepam, Flunitrazepam, and Metabolites in Media 162

Appendix C - Optimized Precursor \& Product lons for Diazepam, Flunitrazepam, and Metabolites 166

Appendix D - Calibration Curves for Cocaine, Fentanyl, and Metabolites in Buffer167 Appendix E - Optimized Precursor \& Product lons for Cocaine, Fentanyl, and Metabolites 172

Appendix F - Calibration Curves for Cocaine, Fentanyl, and Metabolites in Media174 Appendix G - Preliminary Method Development for Methadone. 179

Appendix H - Preliminary Method Development for Methadone. 182 


\section{List of Tables}

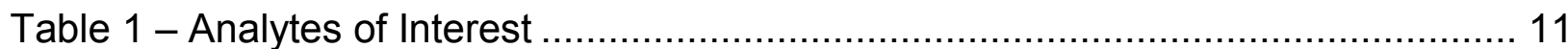

Table 2 - Reinforced Clostridial Medium Components ........................................... 12

Table $3-\mathrm{pH}$ and Viable Microbial Populations in the Human Digestive Tract ............ 22

Table 4 - Prevalent Bacteria in the Lower GI Tract ........................................... 23

Table 5 - Microbial Transmigration Analysis: Time of Appearance ......................... 27

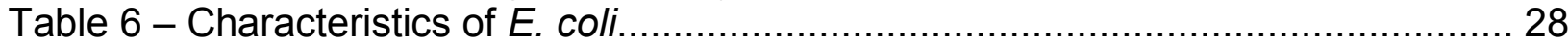

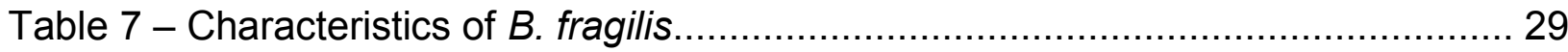

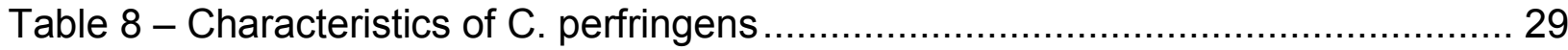

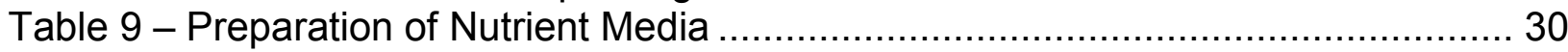

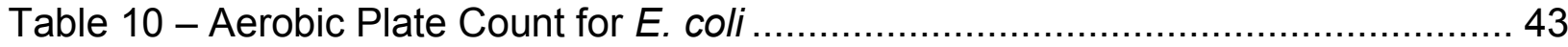

Table 11 - Anaerobic Plate Count for E. coli ................................................ 43

Table 12 - Average Aerobic CFU/mL for E. coli ........................................... 44

Table 13 - Average Anaerobic CFU/mL for E.coli ......................................... 45

Table 14 - Effects of the Microfluidizer on Sample Volume ................................ 47

Table 15 - Volume Differences after Microfluidizer Analysis .................................48

Table 16 - Source and iFunnel Optimization for Diazepam, Flunitrazepam, and

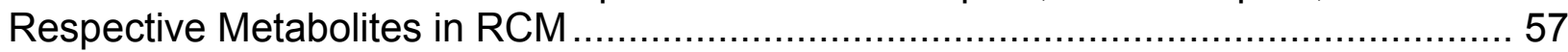

Table 17 - LC-MS/MS Parameters for Diazepam, Flunitrazepam, and Metabolites in

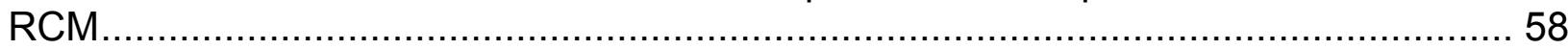

Table 18 - Calibration Range, Correlation, LOD, and LOQ for Diazepam,

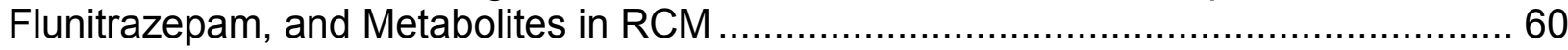

Table 19 - Matrix Effects, Recovery, and Process Efficiency for Diazepam,

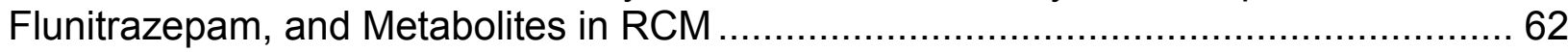

Table 20 - Bias, Within-Run and Between-Run \%CV for Diazepam, Flunitrazepam, and

Respective Metabolites in RCM ............................................................... 65

Table 21 - Dilution Integrity for $1: 2$ and 1:5 Sample Dilutions .............................6 66

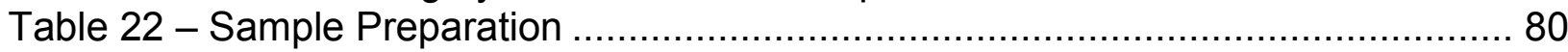

Table 23 - Pre- and Post-Incubation Analysis: Diazepam Drug Blank 1 ................. 83

Table 24 - Pre- and Post-Incubation Analysis: Diazepam Drug Blank 2 .................. 83

Table 25 - Pre- and Post-Incubation Analysis: Effects of E. coli on Diazepam 1 ........ 85

Table 26 - Pre- and Post-Incubation Analysis: Effects of E. coli on Diazepam 2 ........ 86

Table 27 - Pre- and Post-Incubation Analysis: Effects of $B$. fragilis on Diazepam 1.... 87

Table 28 - Pre- and Post-Incubation Analysis: Effects of $B$. fragilis on Diazepam 2.... 87

Table 29 - Pre- and Post-Incubation Analysis: Effects of $C$. perfringens on Diazepam 1

Table 30 - Pre- and Post-Incubation Analysis: Effects of C. perfringens on Diazepam 2

Table 31 - Pre- and Post-Incubation Analysis: Effects of Mixed Culture on Diazepam 1

Table 32 - Pre- and Post-Incubation Analysis: Effects of Mixed Culture on Diazepam 2

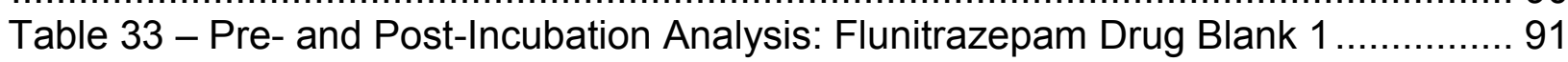

Table 34 - Pre- and Post-Incubation Analysis: Flunitrazepam Drug Blank 2 ............. 91 
Table 35 - Pre- and Post-Incubation Analysis: Effects of E. coli on Flunitrazepam 1 ... 93 Table 36 - Pre- and Post-Incubation Analysis: Effects of E. coli on Flunitrazepam 2 ... 93 Table 37 - Pre- and Post-Incubation Analysis: Effects of $B$. fragilis on Flunitrazepam 1

Table 38 - Pre- and Post-Incubation Analysis: Effects of $B$. fragilis on Flunitrazepam 2 97

Table 39 - Pre- and Post-Incubation Analysis: Effects of C. perfringens on

Flunitrazepam 1

Table 40 - Pre- and Post-Incubation Analysis: Effects of C. perfringens on

Flunitrazepam 2 . 99

Table 41 - Pre- and Post-Incubation Analysis: Effects of Mixed Culture on

Flunitrazepam 1

Table 42 - Pre- and Post-Incubation Analysis: Effects of C. perfringens on

Flunitrazepam 2 .

Table 43 - LC-MS/MS Parameters for Cocaine, Fentanyl, and Respective Metabolites in RCM

Table 44 - Calibration Curve Data, LOD, and LOQ for Cocaine, Fentanyl, and

Respective Metabolites in RCM

Table 45 - Matrix Effects, Recovery, and Process Efficiency for Cocaine and

Metabolites in RCM.

Table 46 - Matrix Effects, Recovery, and Process Efficiency for Fentanyl and Metabolites in RCM

Table 47 - Bias, Within-Run and Between-Run \%CV for Cocaine and Metabolites in

RCM

Table 48 - Bias, Within-Run and Between-Run \%CV for Fentanyl and Metabolites in $\mathrm{RCM}$

Table 49 - Dilution Integrity for Cocaine and Metabolites in RCM 124

Table 50 - Dilution Integrity for Cocaine, Fentanyl, and in RCM 125 


\section{List of Figures}

Figure 1 - Determination of Oxygen Requirements ............................................. 25

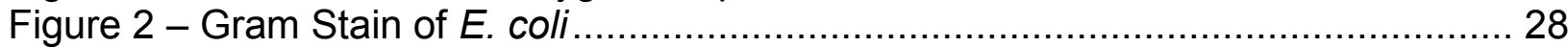

Figure 3 - Coy Laboratory Products, Inc. Type A Vinyl Anaerobic Chamber................ 33

Figure 4 - Schematic of the Type A Vinyl Anaerobic Chamber ................................ 34

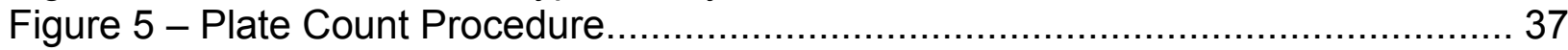

Figure 6 - Serial Dilutions and Spread Plates …….......................................... 42

Figure 7 - Microfluidics M-110P Microfluidizer ................................................. 46

Figure 8 - SPE Method 1\&2 for Diazepam, Flunitrazepam, and Metabolites ............... 53

Figure 9 - SPE Method 3\&4 for Diazepam, Flunitrazepam, and Metabolites ............... 53

Figure 10 - Solid Phase Extraction Vacuum Manifold .......................................... 54

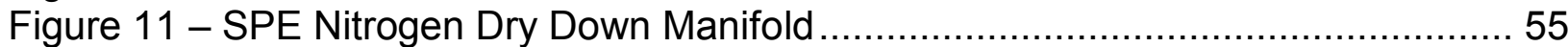

Figure 12 - Agilent G6490 Triple Quadrupole LC/MS....................................... 56

Figure 13 - MRM of Diazepam, Flunitrazepam, and Respective Metabolites in RCM.. 59

Figure 14 - Freeze-Thaw Stability for Diazepam, Flunitrazepam, and Respective

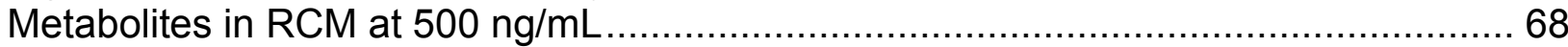

Figure 15 - Freeze-Thaw Stability for Diazepam, Flunitrazepam, and Respective

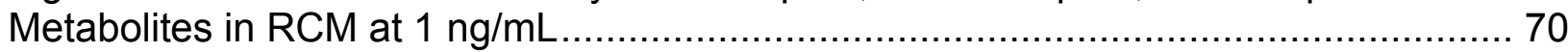

Figure 16 - Processed Sample Stability for Diazepam, Flunitrazepam, and Respective

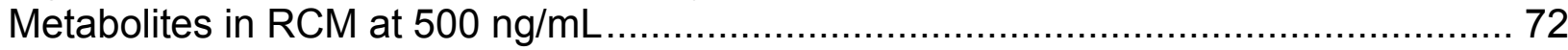

Figure 17 - Processed Sample Stability for Diazepam, Flunitrazepam, and Respective

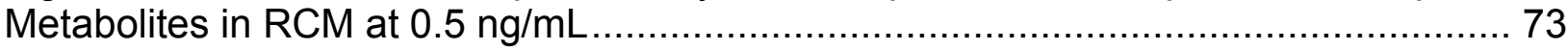

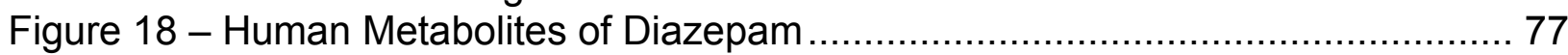

Figure 19 - Human Metabolites of Flunitrazepam .............................................. 78

Figure 20 - Alternative Metabolites of Diazepam and Nordiazepam ........................ 84

Figure 21 - Pre and Post-Incubation Concentrations of Flunitrazepam and Metabolites

Figure 22 - Effects of E. coli on Pre and Post-Incubation Concentrations of

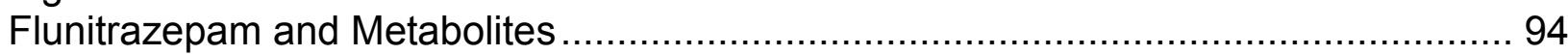

Figure 23 - Conversion of Flunitrazepam to 3-hydroxyflunitrazepam ......................... 95

Figure 24 - Conversion of 7-Aminoflunitrazepam to 7-Acetamino-Flunitrazepam and 7-

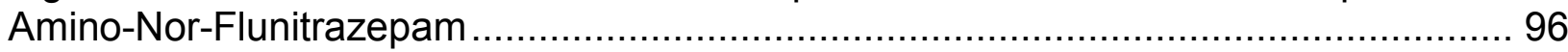

Figure 25 - Effects of $B$. fragilis on Pre and Post-Incubation Concentrations of

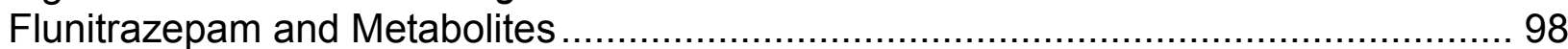

Figure 26 - Effects of $C$. perfringens on Pre and Post-Incubation Concentrations of

Flunitrazepam and Metabolites ......................................................................... 100

Figure 27 - Effects of Mixed Culture on Pre and Post-Incubation Concentrations of

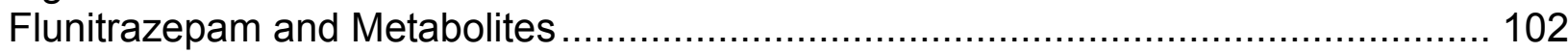

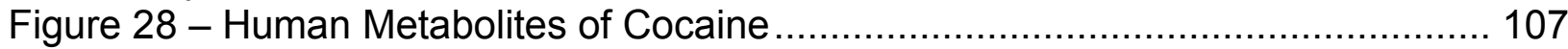

Figure 29 - Human Metabolites of Fentanyl ........................................................ 108

Figure 30 - SPE 1 and SPE 2 for Cocaine, Fentanyl and Metabolites ..................... 110

Figure 31 - SPE 3 for Cocaine, Fentanyl and Metabolites .................................... 111

Figure 32 - MRM of Cocaine, Fentanyl, and Respective Metabolites in RCM............ 115 
Figure 33 - Processed Sample Stability for Cocaine and Metabolites in RCM at High Concentration $(200 \mathrm{ng} / \mathrm{mL})$

Figure 34 - Processed Sample Stability for Fentanyl and Metabolites in RCM at High Concentration $(200 \mathrm{ng} / \mathrm{mL})$..... 128

Figure 35 - Processed Sample Stability for Cocaine and Metabolites in RCM at Low Concentration $(5 \mathrm{ng} / \mathrm{mL})$

Figure 36 - Processed Sample Stability for Fentanyl and Metabolites in RCM at Low Concentration $(5 \mathrm{ng} / \mathrm{mL})$ 


\section{List of Equations}

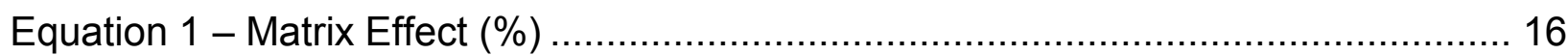

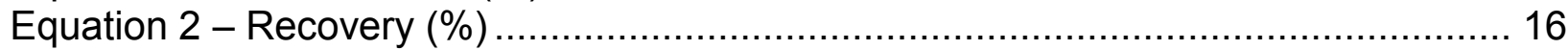

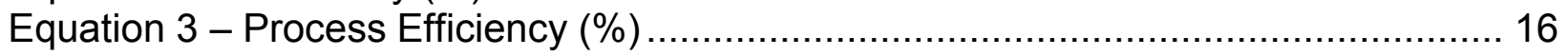

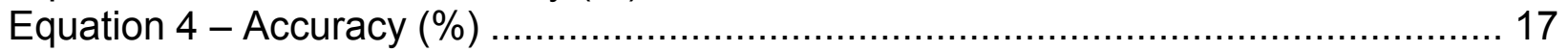

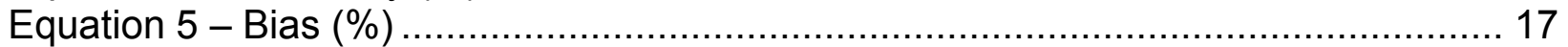

Equation 6 - Within-Run Precision (\%CV) .................................................... 18

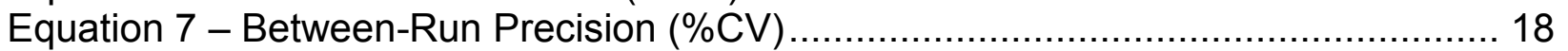

Equation 8 - Determination of Colony Forming Units ................................... 44 


\section{List of Abbreviations, Symbols and Nomenclature}

2,4-DNT 2,4-dinitrotoluene

2,6-DNT 2,6-dinitrotoluene

5AS 5-aminosalicylate

7AM 7-aminoflunitrazepam

ACN Acetonitrile

AEME Anhydroecgonine methyl ester

AEME Anhydroecgonine methyl ester

ATCC American Type Culture Collection

$B$. fragilis Bacteroides fragilis

BBS Biosafety and Biosecurity

BSL-2 Biosafety level 2

BZE Benzoylecgonine

C. perfringens Clostridium perfringens

CDER Center for Drug Evaluation Research

CE Cocaethylene

CF1 Carworth Farms No. 1

CFU Colony forming units

$\mathrm{CH}_{4}$ Methane

CITI Collaborative Institutional Training Initiative

$\mathrm{CO}_{2}$ Carbon dioxide

COC Cocaine

CV Coefficient of variance

CVM Center for Veterinary Medicine

DEA Drug Enforcement Agency

DESP Despropionylfentanyl

DHHS Department of Health and Human Services

DON Deoxynivalenol

DZ Diazepam

E. coli Escherichia coli

EA Ethyl acetate

ECBC Edgewood Chemical Biological Center

EME Ecgonine methyl ester

ESI Electrospray ionization

FA Formic acid

FDA Food and Drug Administration

FENT Fentanyl

FLU Flunitrazepam

FT Freeze-Thaw

g Gram

GC-FID Gas chromatography with flame ionization detector 
GC-MS Gas chromatography - mass spectrometry

GI Gastrointestinal

H1 Rhodococcus sp.

$\mathrm{H}_{2}$ Hydrogen gas

$\mathrm{H}_{2} \mathrm{O}$ Water

$\mathrm{H}_{2} \mathrm{~S}$ Hydrogen sulfide

HPLC High performance liquid chromatography

HPLC-UV High performance liquid chromatography-ultraviolet spectroscopy

hr Hour

$\mathrm{ICH}$ International Conference on Harmonisation

IPA Isopropyl alcohol

LCMS Liquid chromatography mass spectrometry

LC-MS/MS Liquid chromatography - tandem mass spectrometry

LC-UV-MS Liquid chromatography - ultraviolet spectroscopy - mass spectrometry

LOD Limit of detection

LOQ Limit of quantitation

$\mathrm{m} / \mathrm{z}$ Mass-to-charge ratio

M9 M9 Medium

M10 Pseudomonas putida

MB11L Pseudomonas maltophilia

ME Matrix effect

$\mathrm{MeOH}$ Methanol

$\mathrm{mHC}$ m-hydroxycocaine

$\mathrm{mL}$ Milliliter

$\mathrm{mM}$ Millimolar

Mol Molarity

MRM Multiple reaction monitoring

MS Mass spectrometry

MS/MS Tandem mass spectrometry

$\mathrm{N}_{2}$ Nitrogen gas

NC Norcocaine

NDES N-desmethylflunitrazepam

NDZ Nordiazepam

NFENT Norfentanyl

ng Nanogram

$\mathrm{NH}_{3}$ Ammonia

$\mathrm{NH}_{4} \mathrm{OH}$ Ammonium hydroxide

NIH National Institutes of Health

Nitro-PAHs Nitrated polycyclic aromatic hydrocarbons

$\mathrm{nm}$ Nanometers

OD Optical Density

OX Oxazepam

PE Process efficiency 
psi Pounds per square inch

QC Quality control

QQQ Triple quadrupole

$R^{2}$ Correlation coefficient

RCM Reinforced Clostridial Medium

RDECOM Research, Development, and Engineering Command

RE Recovery

SAS Salicylazosulfapyridine

SEM Scanning electron microscopy

SP Sulfapyridine

SPE Solid phase extraction

SPE-LC-ESI- Solid phase extraction-liquid chromatography-electrospray ionizationMS mass spectrometry

SWGTOX Scientific Working Group for Forensic Toxicology

TFTC Too few to count

TIC Total ion chromatogram

TNT 2,4,6-Trinitrotoluene

TNTC Too numerous to count

TS Tryptic Soy Medium

TZ Temazepam

V Volt 


\section{Chapter 1: Introduction}

\subsubsection{Background Information}

Compound detection, identification, confirmation, and quantitation are cornerstone principles of analytical and forensic toxicology. These principles support experimental investigations and applications to innovative areas of research and development.

An exploration of previously published literature and research revealed that bacteria were capable of metabolizing complex chemical substances such as food additives and dyes, environmental contaminants, pharmaceutical compounds, and drugs of abuse. Many of these studies resulted in novel applications toward agricultural and environmental contaminant reduction or elimination, greater insight into the effectiveness of pharmaceutical compounds, or innovative applications of isolated bacterial enzymes.

Some of these same bacterial species are native to the human body and play an active role in the postmortem decomposition process. After death, these species have potential to cause biotransformations that affect the ratio of compound-to-metabolite concentrations within the human body. To date, such postmortem changes have rarely been evaluated or taken into consideration, but they have potential to supply valuable information, especially concerning compound identification and confirmation.

The presented experimental methodology can be adapted and applied to a realm of possibilities. The investigation of new natural products, further study of bacterial 
degradation capabilities for environmental contaminants such as pesticides, explosives, and chemical warfare agents, and isolation and repurpose of native bacterial enzymes for medical use or analyte detection purposes are just the beginning. Ultimately, this methodology would be ideal to study compounds that are too toxic or lethal for animal and human metabolic investigations. This would be particularly useful in defense and military explorations involving exposure to incapacitation and chemical warfare agents, especially in circumstances that result in casualties.

\subsubsection{Agricultural Applications and Environmental Contaminants}

Several studies have investigated the effects of bacterial species on compounds found in food as well as environmental contaminants. Peppercorn and Goldman determined that bacterial species isolated from human feces were capable of converting caffeic acid, which is present in many foods and coffee, to dihydrocaffeic acid and ethylcatechol. ${ }^{1}$ Metabolites were quantified using gas chromatography-mass spectrometry (GC-MS). The authors discovered that individual bacterial species were only able to achieve minimal caffeic acid conversion. However, they noted increased metabolite production in feces for which they concluded conversion of caffeic acid likely resulted from a joint effort amongst the multitude of bacterial species present.

The authors performed a similar set of experiments in which the metabolism of caffeic acid was compared between germfree rats, gnobiotic rats, and conventional rats. ${ }^{2}$ Germfree rats were born and maintained in the sterile environment at the National Institutes of Health $(\mathrm{NIH})$ Germfree Facility. Their diet was supplemented with a $1 \%(\mathrm{w} / \mathrm{w})$ addition of caffeic acid. Urinalysis revealed excretion of intact caffeic acid. 
Gnobiotic rats are those which are born in germfree conditions, but are intentionally inoculated with a specific bacterial specimen or specimens. In this instance, the gnobiotic rats were inoculated with a single strain of Bacteroides sp., Lactobacillus sp.1, Lactobacillus sp.2, or Streptococcus group N strain. The gnobiotic rats inoculated with Streptococcus group $\mathrm{N}$ strain excreted caffeic acid as well as dihydrocaffeic acid. Importantly, these rats were the only ones capable of transforming caffeic acid, and the authors concluded that the Streptococcus group N strain had a direct impact on this metabolic ability. Another set of gnobiotic rats was inoculated with all four strains of bacteria. These rats excreted caffeic acid and dihydrocaffeic acid, as well as two additional metabolites.

Conventional rats exhibited the greatest conversion of caffeic acid to several metabolites. By comparing the metabolites produced by germfree, gnobiotic, and conventional rats, the authors concluded that conversion of caffeic acid is affected by the bacterial species present in the gastrointestinal (GI) tract of the host organism.

Islam et al. isolated a mixture of six bacterial genera including Citrobacter, Clostridium, Enterococcus, Serratia, Stenotrophomonas, and Streptomyces from soil samples collected in crop fields. ${ }^{3}$ This mixed culture converted deoxynivalenol (DON), a contaminant of grains and cereals also known as "vomitoxin", to de-epoxy DON. Conversion occurred rapidly under aerobic conditions, and was monitored using liquid chromatography-ultraviolet-mass spectrometry (LC-UV-MS). The authors proposed that this method be applied to decontamination of tainted grain and corn crops. Methods that use bacterial specimens and their degradation capabilities for decontamination and 
remediation purposes are advantageous because they do not rely on chemical compounds and processes that could result in further environmental problems.

Bharagava and Chandra found that aerobic species Bacillus licheniformis (DQ779010), Bacillus sp. (DQ779011), and Alcaligenes sp. (DQ779012) were capable of biotransforming the compounds that cause distillery wastewater to appear dark brown in color. ${ }^{4}$ Melanoidins are biopolymeric compounds produced by the Maillard reaction, a reaction in which protein and sugar react when heated to produce brown coloration. Caramel-colorants produced in the processing of sugarcane juice as well as distillation of molasses also contributed to wastewater browning. Wastewater samples were inoculated with a mixture of selected aerobic species, glucose, and peptone. Changes in absorbance which correlated with changes in wastewater coloration were monitored with a spectrophotometer.

The bacterial group was capable of converting approximately $70 \%$ of the color compounds. An aliquot of the degraded wastewater sample was purified and analyzed via liquid chromatography-tandem mass spectrometry (LC-MS/MS) to identify and quantify metabolites produced. The authors suggested that their method could be an effective means of environmental decontamination when applied to detoxification of distillery wastewater.

Mohamed discovered a novel bacterial strain denoted Stenotrophomonas maltophilia M1, which was isolated from water sources in Egypt contaminated with pesticide residues. ${ }^{5}$ This strain was capable of degrading methomyl, a toxic pesticide used to control insects and other pests, when glucose was present. Degradation capabilities were analyzed by solid phase extraction-liquid chromatography-electrospray 
ionization-mass spectrometry (SPE-LC-ESI-MS). Mohamed suggested application of M1 in bioremediation of the environment and water sources in areas contaminated with methomyl.

Zhang et al. isolated a bacterial strain capable of efficient nicosulfuron degradation. ${ }^{6}$ Nicosulfuron is a sulfonylurea herbicide used to control weed growth in crop fields and is also a source of groundwater pollution. Authors determined that Serratia marcescens N80 was capable of utilizing nicosulfuron as a sole nitrogen source, the effects for which were measured by liquid chromatography-mass spectrometry (LC-MS). They proposed utilization of N80 in future bioremediation attempts. Further analysis revealed that N80 was also capable of degrading several other sulfonylurea herbicides as well.

Rafii et al. determined that Clostridium clostridiiforme, Clostridium leptum, and Clostridium paraputrificum, amongst others species possessed a nitroreductase capable of converting nitrated polycyclic aromatic hydrocarbons (nitro-PAHs) to various aromatic amines. ${ }^{7}$ Nitro-PAHs are environmental contaminants found in diesel emissions, spent motor oil, and urban air and pose human health risks. Bioconversion decreased the mutagenic effects of the nitro-PAH compounds. The bacterial nitroreductase was isolated and purified, and had potential to be an effective environmental bioremediation agent for nitro-PAH compounds. Rafii and Cerniglia also investigated a Clostridium perfringens azoreductase capable of cleaving azo dyes such as Direct Blue. ${ }^{8,9}$

6-nitrochrysene is another nitro-PAH and known carcinogenic compound. Manning et al. found that intestinal microflora were capable of converting 6- 
nitrochrysene to several metabolites. ${ }^{10}$ However, these products were also thought to have negative toxicological capabilities, which hindered the effectiveness of environmental remediation efforts.

Gallagher et al. discovered a Lysobacter-like bacterium capable of using 2,4,6trinitrotoluene (TNT) as both a carbon and nitrogen source under anaerobic conditions. ${ }^{11}$ TNT is an explosive compound and persistent environmental contaminant. The authors proposed usage of the Lysobacter-like bacterium in future TNT remediation efforts. Similarly, experimental efforts by Nishino, Paoli, and Spain identified a single strain of bacteria capable of degrading 2,4-dintrotoluene (2,4-DNT) as well as three strains capable of degrading 2,6-dinitrotoluene (2,6-DNT) ${ }^{12}$ Both of these compounds are components of TNT and are often seen as soil and water contaminants near former TNT production sites. Combinations of the Lysobacter-like bacterium as well as the species identified by Nishino, Paoli, and Spain may result in more efficient and complete remediation efforts. ${ }^{11,12}$

Kuntze et al. identified Thauera chlorobenzoica as a specimen capable of utilizing organohalides as sole carbon sources under aerobic as well as anaerobic conditions. ${ }^{13}$ These halogenated aromatic compounds are ingredients in insecticides, flame retardants, and lubricants and act as sources of negative human health effects as well as environmental contaminants. T. chlorobenzoica degraded these compounds via denitrification and have potential use in biodegradation efforts of organohalides. 


\subsubsection{Pharmaceutical Compounds}

In addition to the effects on environmental contaminants, several studies demonstrated that some bacterial species are able to transform pharmaceutical compounds. Peppercorn and Goldman performed studies comparing the effects of germfree and conventional rats on salicylazosulfapyridine (SAS). ${ }^{14}$ SAS is a pharmaceutical compound consisting of salicylate and sulfa drug components which act as anti-bacterial and anti-inflammatory agents, respectively. It is often used in the treatment of ulcerative colitis. Conventional rats dosed with SAS excreted urine and feces samples containing the SAS metabolites 5-aminosalicylate (5AS) and sulfapyridine (SP).

The germfree rat did not originally produce these metabolites. However, once removed from the sterile environment, the rat gained the ability to reduce the azo bonds of SAS and produced 5AS and SP. This study demonstrated that GI bacteria are capable of reducing pharmaceutical compounds, which may provide an explanation for differences in human responses to pharmaceutical drugs as well as treatment effectiveness.

Metonidazole is a pharmaceutical compound used in the treatment of trichomonal vaginitis and anaerobic bacterial infections. Koch and Goldman compared results of germfree and conventional rats, and determined that GI bacteria were responsible for the transformation of metonidazole to $\mathrm{N}$-(2-hydroxyethyl)-oxamic acid. ${ }^{15}$ This metabolite was seen in conventional rats but not in their germ-free counterparts. 


\subsubsection{Drugs of Abuse}

A few studies have described the ability of some bacterial species to transform drugs of abuse. Robertson and Drummer studied the effects of eight GI species including Bacteroides fragilis, Bacillus spp., Clostridium perfringens, Escherichia coli, Proteus miribalis, Staphylococcus aureus, Staphylococcus epidermidis, and Streptococcus faecalis on clonazepam, flunitrazepam, and nitrazepam in blood. ${ }^{16}$ Samples were analyzed via high performance liquid chromatography with ultraviolet detector (HPLC-UV). ${ }^{17}$ All three drugs were converted to their respective 7 -amino metabolites though the rate of metabolism varied between species. ${ }^{16}$ Effects of $\mathrm{pH}$ and temperature were examined as well. Changes in $\mathrm{pH}$ affected the rate of metabolism for most species, but there was no discernible trend. However, as temperature increased from $4^{\circ} \mathrm{C}$ to $22^{\circ} \mathrm{C}$ to $37^{\circ} \mathrm{C}$, all eight $\mathrm{GI}$ species demonstrated greater efficiency in metabolism.

Bressler et al. isolated a Rhodococcus sp. strain designated as MB1 from soil near Erythroxylum coca, the plant from which cocaine is produced. ${ }^{18}$ Authors determined that MB1 was capable of utilizing cocaine as a sole source of both carbon and nitrogen. Cocaine was hydrolyzed to form ecgonine methyl ester, a metabolite commonly seen in human metabolic studies, as well as benzoic acid. The authors extracted the Rhodococcus sp. cocaine esterase and proposed to utilize it in the design of a sensor for cocaine detection.

A strain of Pseudomonas maltophilia (MB11L) was also capable of utilizing cocaine as a sole carbon source. ${ }^{19}$ Britt, Bruce, and Lowe isolated this species from industrial waste and performed subsequent analysis. Like the Rhodococcus sp., 
MB11L converted cocaine to ecgonine methyl ester and benzoic acid. Analyses were performed using HPLC-UV and gas chromatography with a flame ionization detector (GC-FID).

Brim et al. developed a mutated Rhodococcus cocaine esterase known as DMCocE. ${ }^{20,21}$ This enzyme hydrolyzed cocaine as well as metabolites norcocaine and cocaethylene, but had no effect on benzoylecgonine. Metabolites were quantified using LC-MS/MS. ${ }^{21}$ The authors aimed to apply the enzyme to the treatment of cocaine addiction as well as in treatment of cocaine toxicity as a result of overdose..$^{20,21}$

Labroo et al. compared the extent and rate of fentanyl metabolism by human liver microsomes and duodenal microorganisms. ${ }^{22}$ Samples were quantified via GC-MS. Both systems converted fentanyl to dealkylated metabolite norfentanyl; however the liver performed the conversion twice as quickly. Cytochrome P450 3A4 was identified as the predominant enzyme responsible for the conversion of fentanyl to norfentanyl in both the liver and duodenum.

Cameron et al. isolated a Rhodococcus sp. $(\mathrm{H} 1)$ from garden soil. ${ }^{23} \mathrm{H} 1$ was capable of utilizing heroin as a sole carbon source, hydrolyzing it into morphine. Authors planned to purify the heroine esterase and combine it with a previously discovered morphine dehydrogenase isolated from Pseudomonas putida (M10) in an effort to create a biosensor for heroin detection.

\subsubsection{Purpose}

The purpose of this research was to investigate the effects of several bacterial species on drugs of abuse, and to compare these metabolites to those produced during 
normal human metabolism. To analyze and quantify these effects, a novel liquid chromatography-tandem mass spectrometry (LC-MS/MS) method was developed for analytes of interest as well as the corresponding human metabolites in Reinforced Clostridial Medium (RCM). The method was used to investigate and analyze the specific effects of human GI species on the targeted drugs of abuse compounds. At the onset of this research, similar methods of extraction and quantitation of drugs of abuse in a nutrient medium matrix and application toward analysis of biotransformation studies had not been published.

\subsubsection{Application}

Comparing the metabolites produced by bacteria to those of human metabolism was considered a particularly interesting area of exploration. This research focused on the effects of GI bacteria, specifically Escherichia coli, Bacteroides fragilis, and Clostridium perfringens on common drugs of abuse. These bacteria were chosen because they are prevalent in the GI tract and have demonstrated biotransformation capabilities which are summarized in sections 1.1.1 - 1.1.3. Preparation and growth of these species are discussed in Chapter 2.

Diazepam (DZ), flunitrazepam (FLU), cocaine (COC), and fentanyl (FENT) were chosen as the initial analytes of interest. They are all classified as drugs of abuse by the United States Drug Enforcement Agency (DEA), Schedule II-Schedule IV, and are therefore of forensic importance. ${ }^{24}$ Drug use and overdose are common occurrences, and drug selection was based upon compounds for which human metabolism studies have been well documented and commercial standards were readily available. Table 1 
depicts the analytes of interest as well as the corresponding structure, formula, and molecular weight.

Table 1 - Analytes of Interest

Analyte




\subsubsection{Analysis}

Analytes were extracted from RCM using solid phase extraction (SPE). RCM is a complex matrix designed to provide the nutrients necessary to promote growth of bacterial species, particularly Clostridia, other anaerobes, and specimens encountered in clinical samples. RCM components are detailed in Table 2. The SPE methods focused on reduction of matrix interferences, particularly the salts and polar components of the media, which could inhibit effectiveness instrumental analysis.

Table 2 - Reinforced Clostridial Medium Components

\begin{tabular}{|c|c|}
\hline Components & Conditions \\
\hline $\begin{array}{ll}\text { - } & 10.0 \mathrm{~g} \mathrm{Beef} \text { Extract } \\
\text { - } & 10.0 \mathrm{~g} \mathrm{Peptone} \\
\text { - } & 3.0 \mathrm{~g} \mathrm{Yeast} \mathrm{Extract} \\
\text { - } & 5.0 \mathrm{~g} \mathrm{Dextrose} \\
\text { - } & 5.0 \mathrm{~g} \mathrm{NaCl} \\
\text { - } & 3.0 \mathrm{~g} \mathrm{NaC} \mathrm{H}_{3} \mathrm{O}_{2} \\
\text { - } & 1.0 \mathrm{~g} \mathrm{Soluble} \mathrm{Starch} \\
\text { - } & 0.5 \mathrm{~g} \mathrm{Cysteine} \mathrm{HCl} \\
\text { - } & 15.0 \mathrm{~g} \text { agar for } \mathrm{RCM} \text { agar }\end{array}$ & $\begin{array}{c}\text { Adjust to } 1.0 \mathrm{~L} \\
\text { Final } \mathrm{pH}=6.8 \pm 0.2 \\
\text { Autoclave } @ 121^{\circ} \mathrm{C} \\
\text { for } 15 \text { minutes. }\end{array}$ \\
\hline
\end{tabular}

LC-MS/MS was chosen as the analytical technique for several reasons. The compounds of interest exhibited structural similarities and displayed similar fragmentation patterns under common analytical processes. High performance liquid chromatography (HPLC) allowed for selection of both solid (column) and mobile phases (buffers) as well as buffer composition, type of elution, flow rate, and column temperature to enhance separation and selectivity of the closely related and structurally similar analytes. Triple quadrupole (QQQ) tandem mass spectrometry (MS/MS) was 
chosen for sensitivity and additional selectivity with mass analysis performed in multiple reaction mode (MRM). Analyte-specific ion transitions were quantified in narrow windows corresponding to retention time.

\subsubsection{Method Validation}

Compound detection, identification, confirmation, and quantitation are cornerstone principles of analytical and forensic toxicology. These principles support development of experimental investigations and applications for innovative areas of research and development. To perform analysis with reliability and certainty, a method must be validated. There are many published guidelines available that discuss validation requirements; however, there is a degree of subjectivity to be evaluated by the analyst. Methods development and validation were discussed in Chapter 3 and Chapter 5 of this research.

For method validation, the intended use of the method should dictate the design of the validation plan as well as parameter selection. Validation parameters should be examined under the same analytical conditions and using the same SPE and instrumental techniques as the final method. Finally, the parameter definitions and validation should be reported so that experiments and analysis could be reasonably reproduced by an alternative analyst or laboratory.

In this research, validation definitions and practices were based upon the International Conference on Harmonisation (ICH) guidelines for linearity, specificity, accuracy, precision, repeatability, detection limit, quantitation limit, range, and

robustness. ${ }^{25}$ The Scientific Working Group for Forensic Toxicology (SWGTOX) 
Standard Practices for Method Validation in Forensic Toxicology ${ }^{26}$ and the Guidance for Industry: Bioanalytical Method Validation ${ }^{27}$ produced by the U.S. Department of Health and Human Services (DHHS), Food and Drug Administration (FDA), Center for Drug Evaluation and Research (CDER), and Center for Veterinary Medicine (CVM) were also used as references.

\subsubsection{Linearity}

Linearity was defined as the method's ability to achieve responses that are directly proportional to analyte concentration within a given range. ${ }^{27}$ A calibration model was used to address the linearity for each analyte of interest. Most guidelines required four to six points in creation of the calibration curve. ${ }^{26,27}$ For purposes of this research, calibration models consisted of a 10-point calibration set prepared and extracted from RCM. The 10-point calibration was chosen because it allowed for adjustment to the model for each analyte based upon the limit of detection and range, while maintaining the requirement of six calibration points. The curve was constructed as a linear model ignoring the origin, and with $1 / x$ weighting. The correlation coefficient $\left(R^{2}\right)$ was used to assess the linearity of each analyte in the method. The calibration model was one of the most important validation parameters assessed. All subsequent analysis and quantitation values are all based upon the relationship established in the calibration model.

\subsubsection{Carryover Effects}

Carryover analysis was conducted for a minimum of three concentration levels

including the highest calibration point. ${ }^{26}$ Two points at concentrations higher than those 
included in the calibration model were also investigated. An extracted blank was injected after each sample and carryover effects were determined by analysis of the blank injections.

\subsubsection{Specificity}

The specificity of a method was defined as the ability to evaluate the analyte of interest in the presence of matrix components or impurities. ${ }^{27}$ Specificity was determined by conducting interference studies as well as assessing ionization suppression/enhancement, matrix effects, recovery, and process efficiency. ${ }^{26}$

\subsubsection{Interference Studies}

Ten samples of RCM matrix were prepared, pooled, and autoclaved at $121^{\circ} \mathrm{C}$ for 15 minutes. Blank samples were extracted and then analyzed to assess possible interferences between matrix components and the analytes of interest. Possible interferences were identified based upon retention time, peak shape, and ion ratios of the target analytes. Ion ratios were calculated as a ratio of response between qualitative ion: quantitative ion. Blank matrix was also spiked with deuterated standards to address possible interferences arising from similar fragmentation patterns of target analyte and corresponding deuterated standard. ${ }^{26}$

\subsubsection{Ionization Suppression/Enhancement and Recovery}

Ionization suppression/enhancement studies were combined with matrix effect $(\mathrm{ME})$, recovery (RE), and process efficiency (PE) studies. Analysis was based on methods published by SWGTOX ${ }^{26}$ and Matuszewski. ${ }^{28}$ Samples were prepared in 3 sets. Set A consisted of neat samples prepared in buffer. Set B consisted of post- 
extraction samples for which $1 \mathrm{~mL}$ of blank matrix was extracted and dried and then spiked with the appropriate amounts of working standard, internal standard, and reconstitution buffer to achieve the desired concentration. In Set C, matrix samples were spiked with analytes and internal standards and then extracted, dried, and reconstituted in $1 \mathrm{~mL}$ of buffer. Samples were analyzed in triplicate. The following equations were used to calculate the percentage ME as determined by ionization suppression or enhancement (Equation 1), RE (Equation 2), PE (Equation 3).

Equation 1 - Matrix Effect (\%)

$$
\% M E=\left(\frac{\text { Set B }}{\text { Set A }}\right) \times 100
$$

Equation 2 - Recovery (\%)

$$
\% \mathbf{R E}=\left(\frac{\text { Set C }}{\text { Set B }}\right) x 100
$$

Equation 3 - Process Efficiency (\%)

$$
\% \mathbf{P E}=\left(\frac{\text { Set C }}{\text { Set } \mathrm{A}}\right) x 100
$$

\subsubsection{Accuracy}

Accuracy was defined as the degree of agreement between the experimental and theoretical values. ${ }^{27}$ Accuracy was calculated using Equation $4 .{ }^{26}$ The acceptable accuracy range was established at $\pm 20 \%(80-120 \%)$ of the theoretical concentration value. 
Equation 4 - Accuracy (\%)

$$
\% \text { Accuracy }=100+\left(\frac{[\text { Experimental }]-[\text { Theoretical }]}{[\text { Theoretical }]}\right) x 100
$$

\subsubsection{Bias}

The bias of the method was also determined by the agreement between the experimental and theoretical values. ${ }^{26}$ However, bias measures the agreement between all the samples at a given concentration level. The $\%$ bias was calculated using Equation 5 where the grand mean of [EXP] (experimental concentration) represents the average of the entire concentration level and [THEO] is equal to the theoretical or known value for that concentration level. The acceptable range for bias was established at $\pm 20 \%$ of the theoretical concentration value.

Equation 5 - Bias (\%)

$$
\% \text { Bias }=\left(\frac{\text { Grand Mean }[\text { EXP }]-[\text { THEO }]}{[\text { THEO }]}\right) \times 100
$$

\subsubsection{Precision}

Precision was defined as the agreement between replicate analyses of the same sample. ${ }^{27}$ The SWGTOX guidelines referred to this as within-run precision. ${ }^{26}$ Data used in bias calculations were also used in precision calculations. The within-run precision was determined by analyzing the triplicate runs for each sample as seen in Equation 6 where [Run] represents the mean of the triplicate runs and $\sigma R$ represents the standard deviation of the mean between the triplicate runs. The within-run precision for each concentration level was considered to be the highest calculated within-run $\%$ 
$\mathrm{CV}$ for each concentration set. The acceptable range for within-run precision was established at $\pm 20 \%$ of the theoretical concentration value.

Equation 6 - Within-Run Precision (\%CV)

$$
\text { Within }- \text { Run } \% \mathbf{C V}=\left(\frac{\sigma \mathbf{R}}{[\text { Run }]}\right) \times 100
$$

\subsubsection{Repeatability}

Repeatability was defined as a measure of agreement between samples in a short period of time under the same operating conditions. ${ }^{27}$ The SWGTOX guidelines refer to this as between-run precision. ${ }^{26}$ Between-run precision was determined by analyzing the 5 runs at the same concentration level. Between-run precision was calculated using Equation 7 where [Level] represents the grand mean of the concentration level and $\sigma \mathrm{L}$ represents the standard deviation of the grand mean of all the runs at each concentration level. The between-run precision for the method was considered to be highest calculated between-run $\mathrm{CV}$ for each concentration level. The acceptable range for between-run precision was established at $\pm 20 \%$ of the theoretical concentration value.

Equation 7 - Between-Run Precision (\%CV)

$$
\text { Between }- \text { Run } \% \mathrm{CV}=\left(\frac{\sigma \mathrm{L}}{[\text { Level }]}\right) \times 100
$$

\subsubsection{Detection Limit}

Detection limit was defined as the concentration at which an analyte can be detected, but is not necessarily able to be quantified. ${ }^{27}$ This was also referred to as the limit of detection (LOD). SWGTOX requirements for LOD were more specific. ${ }^{26}$ The 
LOD was determined to be the lowest concentration for which the analyte demonstrates acceptable peak shape, retention time, and ion ratios as well as a signal response equal to at least three times greater than the background noise response $(S / N \geq 3)$. This was determined for each analyte.

\subsubsection{Quantitation Limit}

The quantitation limit was defined as the lowest concentration for which an analyte can be quantified with acceptable accuracy and precision. ${ }^{27}$ SWGTOX requirements for limit of quantitation (LOQ) included LOD requirements of peak shape, retention time, and ion ratios as well as acceptable accuracy and precision. ${ }^{26}$ This was also calculated for each analyte, the value for which served as the concentration on the calibration model.

\subsubsection{Range}

The range of an analyte was defined as the values between the LOQ and the highest calibration value over which a linear response was maintained. ${ }^{27}$ All method calibration curves we prepared according to these guidelines.

\subsubsection{Dilution Integrity}

Dilution integrity was an important feature of method validation, especially when applied sample concentrations exceeded the calibration range of the analyte of interest. ${ }^{26}$ To determine dilution integrity, bias and precision studies were recalculated for the diluted samples. Dilution integrity was analyzed at 1:2, 1:5, and 1:10 ratios. Five samples per concentration level were prepared, extracted, and analyzed in triplicate. 


\subsubsection{Stability}

\subsubsection{Freeze-Thaw Stability}

Freeze-thaw (FT) stability and processed sample stability were both assessed as part of the method validation. ${ }^{26}$ For freeze-thaw analysis, three samples per concentration were prepared in media and frozen for 24 hours. All of the samples were thawed at room temperature. One vial from each concentration level was extracted and analyzed (FT1) while the other two were frozen for an additional 24 hours. The cycle was repeated for FT2 and FT3. This type of analyte stability was important for samples that could not be analyzed immediately and were frozen for preservation.

\subsubsection{Processed Sample Stability}

The stability of processed samples was studied to address possible effects of large sample batches or instrument malfunctions that could prevent samples from being analyzed immediately. ${ }^{26}$ Stability studies were conducted at two concentrations, high and low. Two samples were prepared in RCM at each concentration, extracted, reconstituted, and analyzed in triplicate over 48-60 hours.

\subsubsection{Robustness}

Robustness of a method is defined as the ability to remain unaffected as slight variations are made to method parameters. ${ }^{25,26}$ Robustness of the method was determined by slight changes in the buffer composition and column temperature. In addition to buffer composition and column temperature, an alternate analyst prepared samples for analysis. 


\section{Chapter 2: Optimization of Bacterial Growth and Preparation}

\subsubsection{Introduction}

Anaerobic bacteria endogenous to the human body are active in the postmortem decomposition process. To study the effects of these species on the selected drugs of abuse, bacterial methods were developed and optimized. A nutrient medium, environmental and growth conditions, and methods of cellular lysis were selected and optimized. A frozen stock culture was prepared for future experiments and a serial dilution and plate count method was used to estimate the number of viable bacteria.

\subsubsection{Background}

Cellular metabolism is estimated to continue for approximately 4-10 minutes postmortem. ${ }^{29}$ During this interval, carbon dioxide $\left(\mathrm{CO}_{2}\right)$ begins to accumulate which lowers $\mathrm{pH}$. This continues until the acidity reaches concentrations capable of rupturing cellular membranes. Lysing of these membranes causes the release of digestive enzymes, and marks the beginning of the decomposition process. ${ }^{29-31}$

The decomposition process is typically broken down into four stages: autolysis, putrefaction, decay, and diagenesis. ${ }^{30,31}$ In the autolysis or self-digestion phase, cellular enzymes begin to dissolve the remaining cellular structures from within. These chemical changes within the cell along with decreasing oxygen and increasing carbon dioxide cause waste to accumulate and poison the cell. Resulting fluids from these processes lead to putrefaction. Putrefaction is marked by catabolism of the soft tissue 
by endogenous bacteria and microbes, especially those native to the GI tract. The catabolic processes result in production of various gases such as ammonia $\left(\mathrm{NH}_{3}\right)$, methane $\left(\mathrm{CH}_{4}\right)$, carbon dioxide $\left(\mathrm{CO}_{2}\right)$, and hydrogen sulfide $\left(\mathrm{H}_{2} \mathrm{~S}\right)$ as well as a variety of liquid byproducts. After the soft tissues have been broken down, the body enters decay in which remaining protein and fats are decomposed. The final stage of decomposition is diagenesis and is marked by the degradation of bones. Anaerobic bacteria and microbes endogenous to the human body are active in the postmortem decomposition process, especially during the putrefaction and decaying phases. ${ }^{30,31}$

Indigenous microflora colonize much of the human body both on the surface and internally. Though the intestinal tract is sterile at birth, a "mature" microfloral environment is established within 3-4 weeks of life. The numbers and types of bacteria differ between regions of the body based upon $\mathrm{pH}$ conditions and the oxygen requirements of the microbes themselves. ${ }^{32,33}$

Table 3 lists approximations of the number of viable bacteria in each section of the GI tract per gram of sample taken. ${ }^{32-34}$ While a total bacterial count includes both living and dead organisms, the viable bacteria count estimates the number of bacteria present that are living and able to both grow and divide; thus, capable of metabolic activity. ${ }^{32,33}$

Table $3-\mathrm{pH}$ and Viable Microbial Populations in the Human Digestive Tract

\begin{tabular}{|ccccc|}
\hline & Stomach & Jejunum & Ileum & Colon \\
\hline Viable Bacteria/Gram & $0-10^{3}$ & $0-10^{4}$ & $10^{5}-10^{8}$ & $10^{10}-10^{12}$ \\
\hline $\mathrm{pH}$ & 3.0 & $6.0-7.0$ & 7.5 & $6.8-7.3$ \\
\hline
\end{tabular}


This research focuses on the lower GI tract, particularly the ileum and colon. In these sections, the oxygen concentration is limited, which promotes the largest populations of facultative anaerobic and anaerobic species. Table 4 contains information pertaining to the most abundant facultative anaerobic and obligate anaerobic bacteria endogenous to the human intestinal tract. It also includes basic information pertaining to Gram-stain, morphology, and oxygen requirements for each species. ${ }^{32,35}$

Table 4 - Prevalent Bacteria in the Lower GI Tract

\begin{tabular}{|cccc|}
\hline Bacteria & Gram & Morphology & O $_{2}$ Requirements \\
\hline Bacteroides sp. & - & Bacillus & Obligate Anaerobe \\
\hline Clostridium sp. & + & Bacillus & Obligate Anaerobe \\
\hline Enterococcus faecalis & - & Coccus & Facultative Anaerobe \\
\hline Escherichia coli & - & Bacillus & Facultative Anaerobe \\
\hline Proteus sp. & - & Bacillus & Facultative Anaerobe \\
\hline Staphylococcus aureus & + & Coccus & Facultative Anaerobe \\
\hline Staphylococcus epidermidis & + & Coccus & Facultative Anaerobe \\
\hline Streptococcus mitis & + & Coccus & Facultative Anaerobe \\
\hline Streptococcus pyogenes & + & Coccus & Facultative Anaerobe \\
\hline Enterobacteriacea & - & Bacillus & Microaerophile/Anaerobe \\
\hline Lactobacillus sp. & + & Bacillus & Microaerophile/Anaerobe \\
\hline
\end{tabular}

The Gram-stain is commonly used as the starting point in classification and identification of bacterial samples. ${ }^{34-36}$ It is a differential staining technique, which distinguishes between Gram-positive and Gram-negative specimens based upon structural differences in the cell walls. Gram-positive bacteria have more disaccharides and amino acids which form a thicker peptidoglycan layer and stain purple, while Gramnegative bacteria have a lipopolysaccharide layer which consists of lipids and polysaccharides that stains pink. The Gram staining procedure is also useful for 
determining the morphology or shape of a specimen as well. The morphology of a bacterium is used in further classification and identification. There are three categories: coccus specimens who are spherical, bacillus specimens who are rod-shaped, and spiral specimens.

Bacteria are classified into five categories based upon requirement for and tolerance of atmospheric oxygen. ${ }^{34-36}$ Aerobic species are those which require abundant oxygen for metabolic processes and growth, while microaerophilic species require only a finite amount of oxygen and can be metabolically hindered or even killed by excessive amounts. Facultative anaerobes prefer oxygenated environments for aerobic respiration, but are also capable of cellular respiration and fermentation under limited oxygen or anaerobic conditions. Aerotolerant anaerobic species are fermentative and prefer oxygen-poor environments, but can tolerate the presence of oxygen, while obligate anaerobic species require the absence of oxygen. ${ }^{34,36} \mathrm{~A}$ depiction of oxygen requirements can be seen in Figure $1 .^{34}$ Though bacteria from all five categories can be found in the human body, the oxygen content of the GI region is strictly limited or absent. Therefore, the bacteria of interest for the purposes of this research are facultative or obligate anaerobes. 
Figure 1 - Determination of Oxygen Requirements

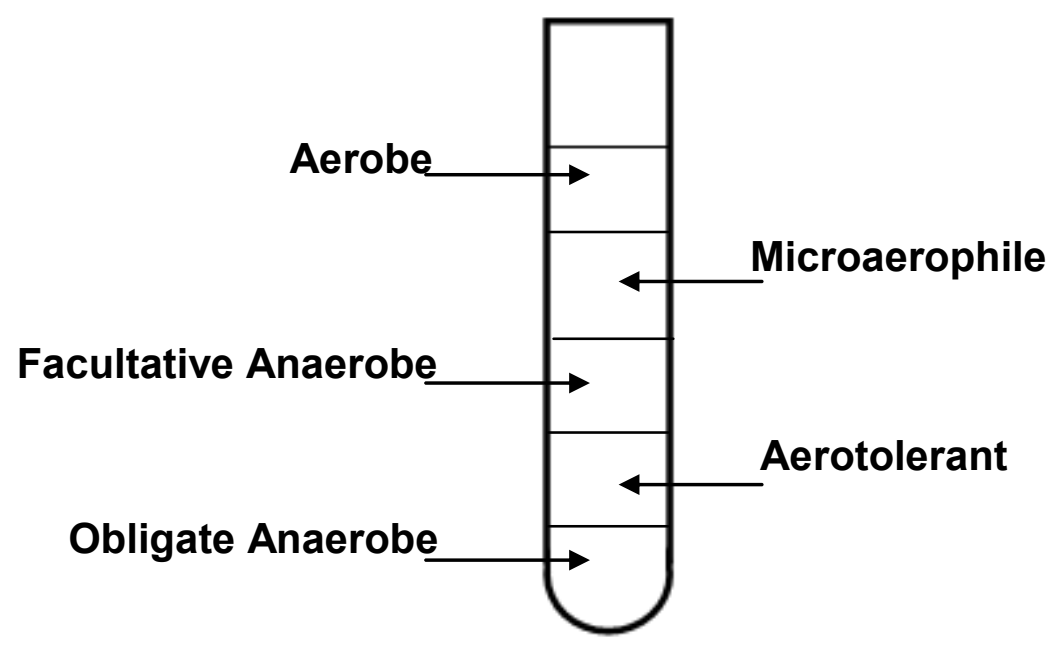

Three of the bacterial species discussed in Table 4 were chosen for analysis and include Escherichia coli (E. coli), Bacteroides fragilis (B. fragilis), and Clostridium perfringens (C. perfringens). E. coli was chosen because it is commonly used in microbial research. It is also a facultative anaerobic species highly concentrated in the human gastrointestinal tract. Since the oxygen concentration is severely limited in the ileum and colon, the two obligate anaerobic species $B$. fragilis and $C$. perfringens were also chosen for observation in this research. Effects of these bacterial specimens on the targeted drugs of abuse were investigated. Results are discussed in Chapter 4 .

Postmortem, the populations of GI microflora are capable of otherwise inhibited transmigration. ${ }^{37}$ The body's protective mechanisms both physical (membranes) and chemical ( $\mathrm{pH}$ conditions) are compromised. Bacteria are therefore no longer limited to their native habitats within the body, and are allowed access to areas that were previously protected, including the bloodstream and contents of the stomach to name a few. 
Melvin et al. conducted a series of experiments using a combination of culturing techniques and scanning electron microscopy (SEM) to evaluate bacterial transmigration. ${ }^{37}$ In these experiments, a Carworth Farms No. 1 (CF-1) mouse was decapitated and the small intestine removed. The intestine was divided into three sections. The first section, the "control", was immediately placed in a cold solution of $2.5 \%$ buffered glutaraldehyde, and was used to assess tissue and environmental conditions of the sample immediately postmortem. The middle section of the small intestine was cauterized and stored in solutions of phosphate-buffered saline. The third section was used to evaluate the types of endogenous microbial organisms present via culturing techniques.

The preparations were incubated at $4^{\circ} \mathrm{C}, 25^{\circ} \mathrm{C}$, and $37^{\circ} \mathrm{C}$ to ascertain the effect of temperature on the rate of bacterial transmigration. Samples of the gut were taken at intervals over a 72 hour period for each temperature study. Fixed tissue samples of the gut were dehydrated in ethanol, dried, coated with gold, and examined with the SEM. Aliquots of the saline solution containing the middle section of the small intestine were taken at the same intervals for culturing.

SEM analysis revealed that microbes migrated to the surface of the gut tissue within 2-3 hours at $37^{\circ} \mathrm{C}$. Appearances were delayed to $5-6$ hours when incubated at $25^{\circ} \mathrm{C}$ and 72 hours at $4^{\circ} \mathrm{C}$. Culture analysis demonstrated that rate of migration also depended upon the type microbial organism. For example, Staphylococcus species were some of the first organisms cultured, regardless of temperature. The authors attributed this to the high concentrations of collagenase and protease enzymes inherent 
to these species. Fungi, coliforms, and facultative anaerobic species were cultured in later samples.

Table 5 was adapted from the results of Melvin et al. ${ }^{37}$ This study indicated that many of the bacteria endogenous to the human intestine would be capable of transmigration within 72 hours postmortem, regardless of temperature.

Table 5 - Microbial Transmigration Analysis: Time of Appearance

\begin{tabular}{|cccc|}
\hline Temperature & Staphylococcus Species & Coliforms/Fungi & Coliforms/Anaerobes \\
\hline $\mathbf{4}^{\circ} \mathbf{C}$ & $66-88$ Hours & $68-72$ Hours & $>72$ Hours \\
\hline $\mathbf{2 5 ^ { \circ } \mathbf { C }}$ & $5-6$ Hours & $8-10$ Hours & $12-16$ Hours \\
\hline $\mathbf{3 7}^{\circ} \mathbf{C}$ & $2-3$ Hours & $4-5$ Hours & $6-8$ Hours \\
\hline
\end{tabular}

\subsubsection{Bacterial Selection}

E. coli, B. fragilis, and C. perfringens were all classified as Biosafety Level 2 (BSL-2) organisms. ${ }^{38}$ BSL-2 specimens are those which act as a potentially moderate threat to both the environment as well as personnel. ${ }^{39}$ Each of the organisms was specifically chosen due to human origin, and were ordered from the American Type Culture Collection (ATCC). ${ }^{38}$ The specific characteristics for E. coli 33985 are listed in Table 6. 


\subsubsection{Bacterial Characteristics}

Table 6 - Characteristics of E. coli

\begin{tabular}{|cc|}
\hline ATCC Number & $3^{3985^{\mathrm{TM}}}$ \\
\hline Organism & Escherichia coli (Migula) Castellani and Chalmers \\
\hline Designation & B7 \\
\hline Isolation & Feces (Human) \\
\hline Biosafety Level & BSL-2 \\
\hline Antigenic Properties & Serotype O86a:K61 \\
\hline
\end{tabular}

E. coli is a Gram-negative, rod shaped bacterium. ${ }^{38}$ It can be seen in various arrangements including singles, pairs, and short chains. Size ranges between $0.5 \mu \mathrm{m}$ in width and 1.0-3.0 $\mu \mathrm{m}$ in length. A Gram stain of E.coli can be seen in Figure 2. Colonies are opaque gray/white while broth specimens are generally turbid with sediment. E. coli can act as an anaerobic microbe as well as a facultative anaerobe. Possible growth temperatures range from $10-45^{\circ} \mathrm{C}$, optimally between $30-37^{\circ} \mathrm{C}$.

Figure 2 - Gram Stain of E. coli Reproduced with permission by Gary E. Kaiser

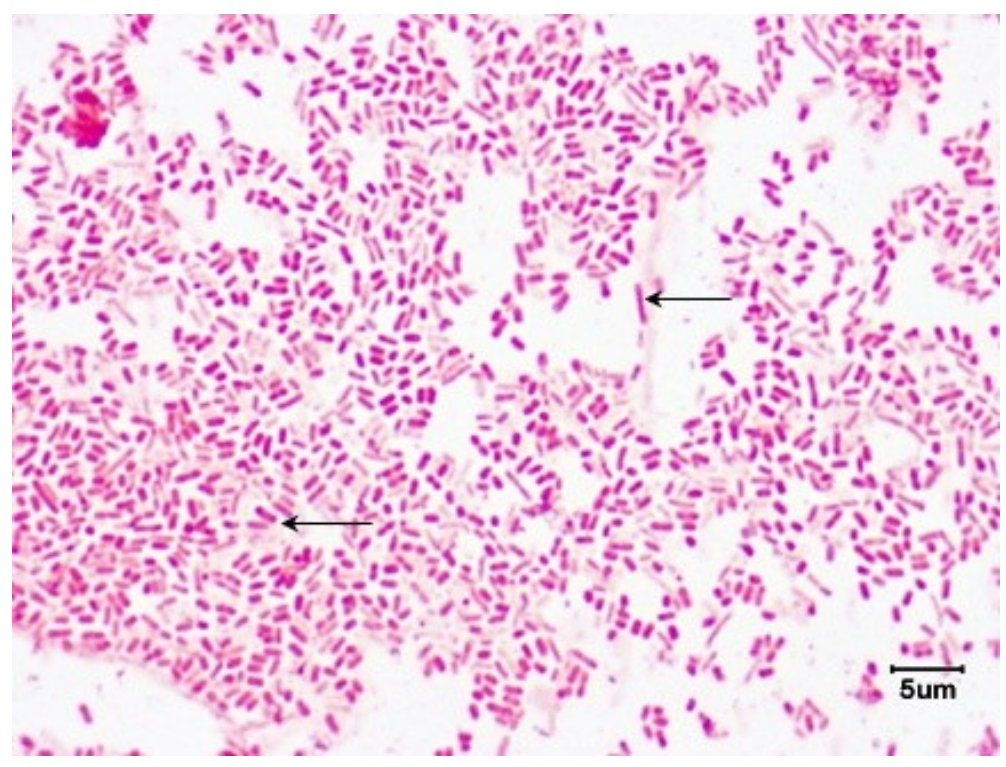


$B$. fragilis is a Gram-negative, rod shaped bacterium commonly seen in singles or pairs. ${ }^{38}$ Size ranges between $0.5 \mu \mathrm{m}$ in width and $1.0-3.0 \mu \mathrm{m}$ in length. Colonies tend to be small, gray, and irregular, and broth specimens are turbid. $B$. fragilis is an anaerobic species with optimal growth at $37^{\circ} \mathrm{C}$. Table 7 shows characteristic of the $B$. fragilis specimen selected from the ATCC. ${ }^{38}$

Table 7 - Characteristics of $B$. fragilis

\begin{tabular}{|cc|}
\hline & 29771 $^{\text {TM }}$ \\
\hline ATCC Number & Bacteroides fragilis (Veillon and Zuber) Castellani and Chalmers \\
\hline Organism & 2044; [CDC 1261; M-488] \\
\hline Designation & Blood \\
\hline Isolation & BSL-2 \\
\hline Biosafety Level & \\
\hline
\end{tabular}

C. perfringens is a Gram-positive, rod shaped bacterium commonly seen in singles or pairs. ${ }^{38}$ Size ranges between $1.0-1.5 \mu \mathrm{m}$ in width and $4.0-8.0 \mu \mathrm{m}$ in length. Colonies are circular and slightly raised with an opaque center. Broth specimens are usually turbid with sediment. C. perfringens is an encapsulated and spore forming anaerobic species. Growth is possible up to $50^{\circ} \mathrm{C}$ with optimal growth between 35$37^{\circ} \mathrm{C}$. Table 8 shows the $\mathrm{C}$. perfringens specimen chosen from the ATCC catalog. ${ }^{38}$

Table 8 - Characteristics of C. perfringens

\begin{tabular}{|cc|}
\hline ATCC Number & $1^{19574}{ }^{\text {TM }}$ \\
\hline Organism & Clostridium perfringens (Veillon and Zuber) Hauduroy et al. \\
\hline Designation & $43 \mathrm{~F} 4$ \\
\hline Isolation & Bile (Human) \\
\hline Biosafety Level & BSL-2 \\
\hline
\end{tabular}




\subsubsection{Media Selection}

Several types of nutrient media were evaluated. Components and conditions for M9 Medium (M9M) and tryptic soy medium (TS) were described in Table 9. ${ }^{38,40}$ Components and conditions of RCM were previously discussed in Table 2. Information about M9 salts, modified Wolin salts, and M9M were obtained from Melissa Dixon at Edgewood Chemical Biological Center. ${ }^{41}$

Table 9 - Preparation of Nutrient Media

\begin{tabular}{|c|c|c|}
\hline Medium & Components & Conditions \\
\hline M9 Salts & $\begin{array}{ll} & 64.0 \mathrm{~g} \mathrm{Na}_{2} \mathrm{HPO}_{4} \cdot 7 \mathrm{H}_{2} \mathrm{O} \\
\text { - } & 15.0 \mathrm{~g} \mathrm{KH}_{2} \mathrm{PO}_{4} \\
\text { - } & 2.5 \mathrm{~g} \mathrm{NaCl} \\
\text { - } & 5.0 \mathrm{~g} \mathrm{NH}_{4} \mathrm{Cl} \mathrm{DI} \mathrm{H} \\
2\end{array}$ & Adjust to $1.0 \mathrm{~L}$ \\
\hline Modified Wolin Salts & 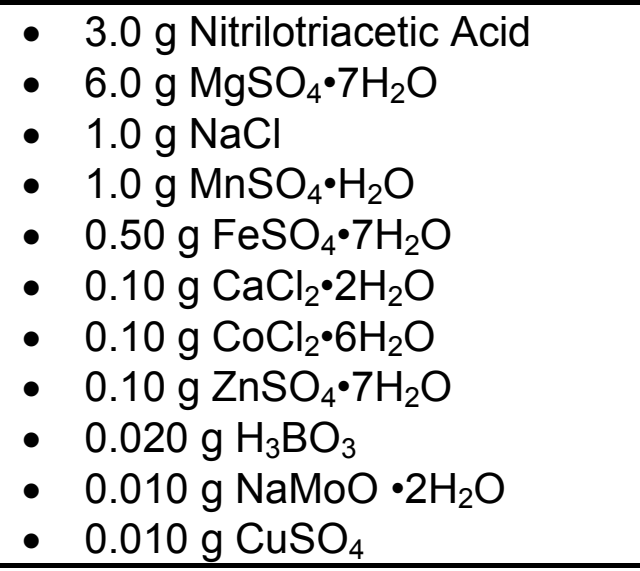 & Adjust to $1.0 \mathrm{~L}$ \\
\hline M9M & $\begin{array}{ll}\text { - } & 200.0 \mathrm{~mL} \text { M9 Salts } \\
\text { - } & 2.0 \mathrm{~mL} 1 \mathrm{M} \mathrm{MgSO}_{4} \\
\text { - } & 0.10 \mathrm{~mL} 1 \mathrm{M} \mathrm{CaCl}_{2} \\
\text { - } & 10.0 \mathrm{~mL} \text { Modified Wolin Salts } \\
\end{array}$ & Adjust to $1.0 \mathrm{~L}$ \\
\hline TS & 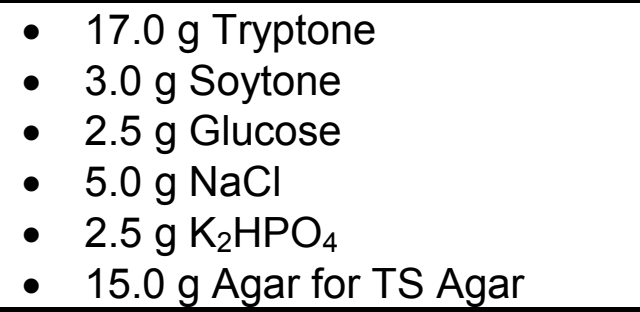 & $\begin{array}{l}\text { Adjust to } 1.0 \mathrm{~L} \\
\text { Autoclave @121으 } 12 \\
\text { for } 15 \text { minutes }\end{array}$ \\
\hline
\end{tabular}


M9M was prepared and then filtered to sterilize. The broth of RCM and TS were prepared and autoclaved for sterilization. A tube containing $10 \mathrm{~mL}$ of broth was inoculated with either E. coli, B. fragilis, or C. perfringens. This was performed for all broth and bacteria combinations. The tubes were incubated for 24 hours at $37^{\circ} \mathrm{C}$ under anaerobic conditions. None of the bacterial species demonstrated growth in M9M. This was attributed to the fact that M9M is a limited nutrient medium. Bacteria require sources of carbon and nitrogen for growth, which are absent in M9M. However, this medium would be ideal for sole source studies.

E. coli demonstrated growth in TSB as evidenced by the cloudy nature of the broth post-incubation. However, the tubes inoculated with B. fragilis and C. perfringens remained the clear yellowish-brown of freshly sterilized TSB. This indicated bacterial growth for these species was not supported by TSB, making it a poor choice for experimental medium. RCM supported and promoted growth of all three species and was therefore selected as the matrix for all of the experimental procedures.

\subsubsection{Certificates and Training}

Specific certifications and training were required for those handling or working with BSL-2 agents as well as for the laboratory itself. ${ }^{39}$ WVU required approval and clearance from the Director of Biological Safety for research involving biohazards. WVU also required biosafety training through the Collaborative Institutional Training Initiative (CITI) program, which offered a Biosafety and Biosecurity (BSS) course. ${ }^{42}$ In addition, The Centers for Disease Control and Prevention (CDC) also recommended a Laboratory Biosafety Training online course. ${ }^{43}$ 
Edgewood Chemical Biological Center (ECBC), a facility under the United States Army Research, Development and Engineering Command (RDECOM) required completion of the Effective Engineering Controls for Biological and Chemical Operations training set as well as the ECBC Visitor Risk Assessment for Chemical and Biological Non-Surety Laboratories. The training requirements were fulfilled prior to commencement of the dissertation research.

The experimental research was performed in the research labs of Dr. Suzanne Bell at West Virginia University as well as the facilities of Edgewood Chemical and Biological Center on the Aberdeen Proving Ground under Dr. Frederic Berg, Dr. Steve Harvey, and Melissa Dixon. All of these facilities obtained the necessary permissions for the BSL-2 human specimen and bacterial work.

\subsubsection{Anaerobic Conditions}

Growth conditions and requirements for the anaerobes are specific. Therefore, methods were developed to ensure optimal growth for each species. Coy Laboratory

Products, Inc. specializes in various types of anaerobic chambers. ${ }^{44}$ The Vinyl Anaerobic Chamber is specifically designed to meet the needs for anaerobic microbiology research and can be seen in Figure 3. 
Figure 3 - Coy Laboratory Products, Inc. Type A Vinyl Anaerobic Chamber Reproduced with permission from Brian Coy of Coy Laboratory Products, Inc.

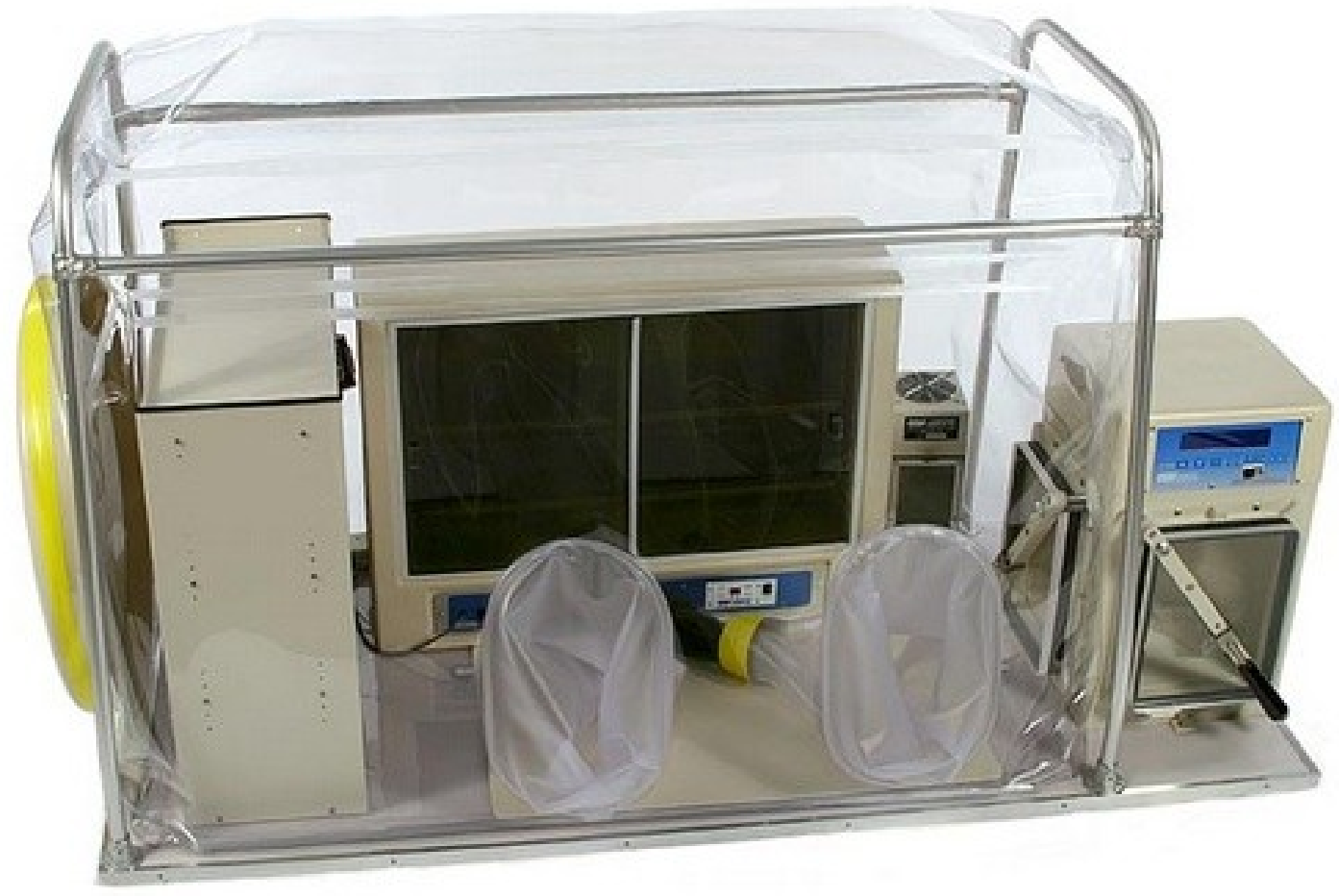

All models are equipped with an airlock system, gas regulator, catalyst fan boxes, 2 stak-paks with catalyst, a gas leak detector, and a plug strip for electronics (Coy Laboratory Products). The Type A Chamber with automatic airlock and an incubator was chosen for this set of research experiments. A schematic of the chamber setup is depicted in Figure 4. 
Figure 4 - Schematic of the Type A Vinyl Anaerobic Chamber

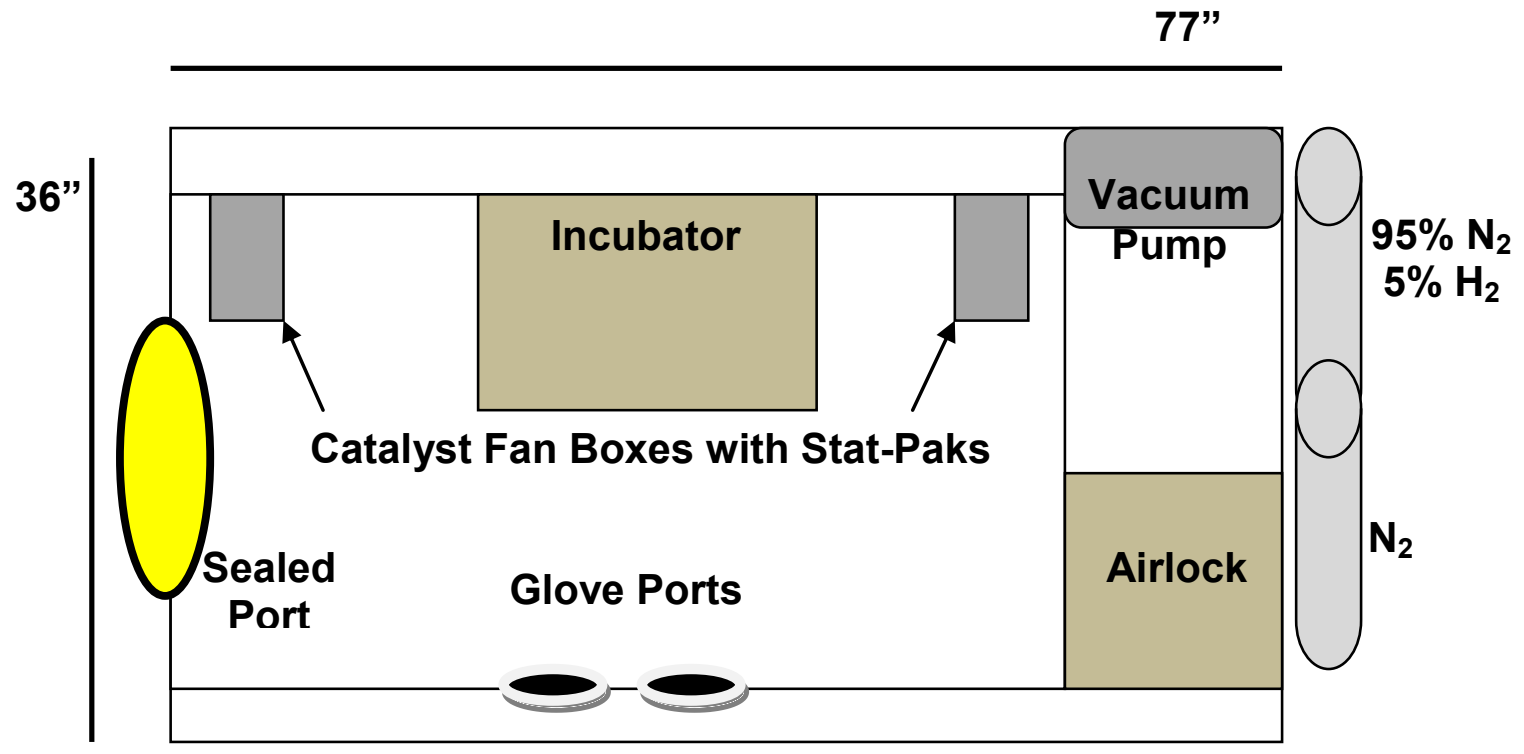

Coy's airlock and chamber systems require a combination of two gases. A background gas, $\mathrm{N}_{2}$, was used to purge most of the oxygen out of the system through the vacuum pump and to inflate the chamber. The second gas was a mixture of $95 \% \mathrm{~N}_{2}$ and $5 \%$ of $\mathrm{H}_{2}$. The $\mathrm{H}_{2}$ combined with $\mathrm{O}_{2}$ molecules within the chamber to form $\mathrm{H}_{2} \mathrm{O}$ molecules. The stat-pak in each catalyst fan box contained a palladium catalyst which facilitated this reaction, and were switched out every 24 hours and dried in a laboratory oven to remove water and ensure optimal performance.

The vinyl chamber offered several advantages over the aluminum and polymer versions. The vinyl chamber required less combination gas consumption and anaerobic conditions were easier to maintain because of the ability of the walls to expand and contract. In addition, vinyl chambers have the lowest cost-per-life cycle of all the models. 


\subsubsection{Frozen Stock Culture (3 Days)}

Prior to use, the ATCC cultures were stored at manufacturer recommended temperature, $-80^{\circ} \mathrm{C}$. To ensure an adequate supply of bacteria for experimental procedures, a set of 10 stock cultures was prepared for each of the three species. On day one, the plastic vial containing the lyophilized bacterial sample was removed from the protective packaging and placed in the anaerobic chamber. A $1 \mathrm{~mL}$ aliquot of sterile water was added to the vial. Contents were vortexed and then transferred to a sterile BD Falcon ${ }^{\mathrm{TM}}$ tube $(15 \mathrm{~mL})$ containing $10 \mathrm{~mL}$ of $\mathrm{RCM}$ and incubated at $37^{\circ} \mathrm{C}$. This was repeated for all three bacterial specimens. After 48 hours of incubation, tubes were removed from the incubator and examined for growth. All three samples produced turbid cultures with a layer of bubbles as well as what appeared to be a gelatinous mixture that was present in all of the tubes. This was identified as a small amount of agar, a component of RCM, which provided support to the anaerobic cultures. It solidified to a slight extent during incubation. After the samples were vortexed, the gelatinous portion mixed back in with the RCM broth.

A $500 \mathrm{uL}$ aliquot of $15 \%$ sterile glycerol in water was transferred into 10 small Eppendorf tubes per bacterial species. The RCM culture tubes were removed from the incubator and vortexed for 30 seconds. A $500 \mathrm{uL}$ aliquot of this solution was transferred into each of the glycerol-water tubes. The tubes were then sealed, gently vortexed to mix, and nine of them stored at $-80^{\circ} \mathrm{C}$ for future use. 


\subsubsection{Plate Count Procedure (5 Days)}

There are many different methods used in the enumeration of bacteria or other microorganisms in a sample. Direct microscope counts, electronic cell counters, and spectrophotometric analysis are all commonly used. ${ }^{34,36}$ However, while these methods are capable of approximating the number of organisms within a sample, they offer one major disadvantage. They are programmed to count the number of living, viable cells as well as any dead cells within the sample. ${ }^{36}$ For this set of experiments, it was important to only approximate the number of viable cells within the sample since these are the ones capable of metabolism. Therefore, the serial dilution-agar plate method was used to estimate the number of viable bacteria within a sample following a given set of experimental conditions. This method was applied to each of the target bacterial species using RCM agar plates and RCM broth.

The plate count procedure required five consecutive days to complete. A summary of the tasks performed on each day was depicted in Figure 5. 
Figure 5 - Plate Count Procedure

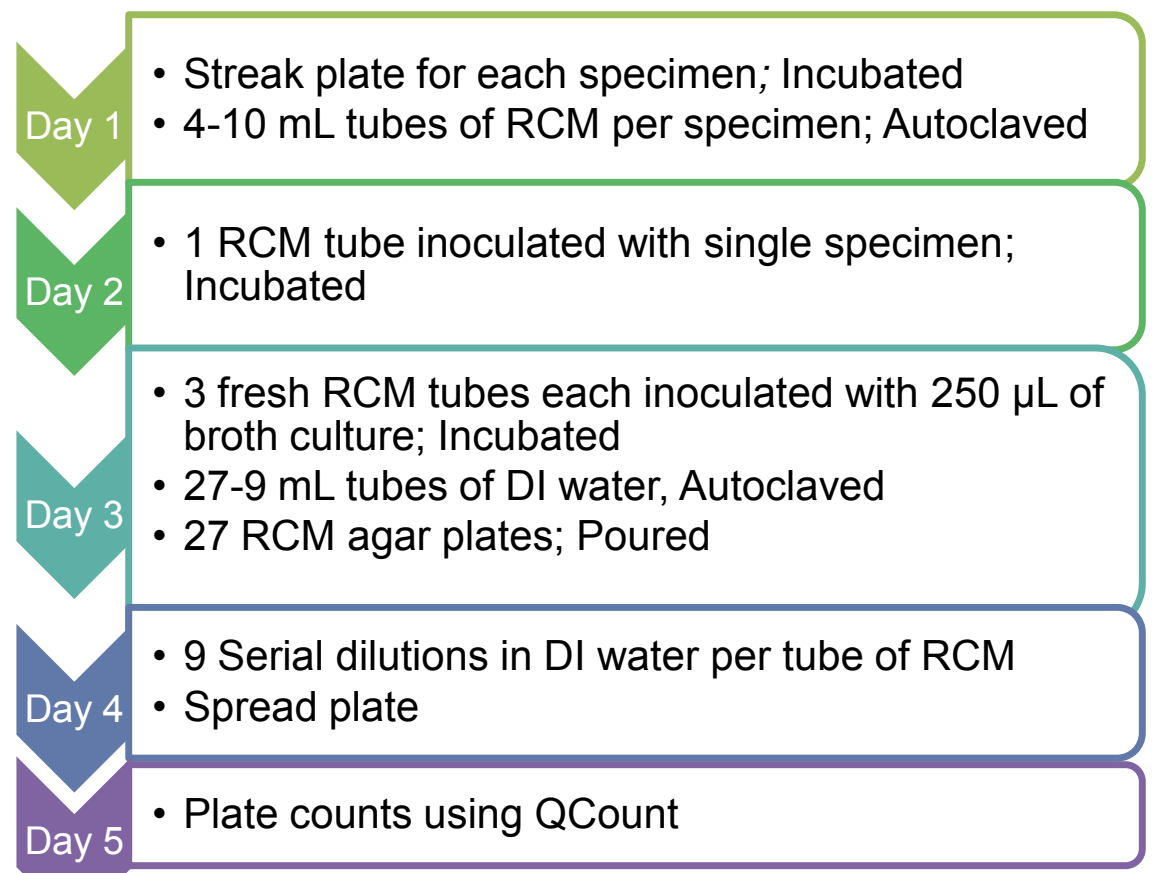

\subsubsection{Plate Count Procedure Day 1}

On Day 1, streak plates for E. coli, B. fragilis, and C. perfringens were prepared from the frozen stock cultures. The RCM agar plates were removed from the refrigerator and placed in the incubator set at $37^{\circ} \mathrm{C}$ for 15 minutes to remove the chill. One tube of frozen stock culture per species was moved from the $-80^{\circ} \mathrm{C}$ freezer and placed in the anaerobic chamber. An inoculation loop was dipped into the frozen stock culture and the agar plate was streaked using the standard streak plate procedure for isolated colonies. Plates were incubated upside down at $37^{\circ} \mathrm{C}$ for 24 hours under anaerobic conditions. Incubating plates upside down ensured that colony formation and growth were not interrupted by condensation that often forms on the lids of the plates during incubation. In addition, four test tubes per specimen, each containing $10 \mathrm{~mL}$ of 
RCM broth were autoclaved at $121^{\circ} \mathrm{C}$ for 15 minutes. After sterilization, the tubes were stored on the bench top at room temperature until they were needed in Day 2 and Day 3.

\subsubsection{Plate Count Procedure Day 2}

On Day 2, plates were examined for colony growth. The E. coli plate was full of small, isolated, circular colonies. However, the B. fragilis and C. perfringens plates were blank and looked as though they had not been previously streaked. An ATCC representative suggested that the anaerobic specimens can often require 24-48 hours for colony formation, so those plates were returned to the incubator for another 24 hours at $37^{\circ} \mathrm{C}$. An isolated colony of $E$. coli was transferred to a test tube of RCM. This tube was incubated at $37^{\circ} \mathrm{C}$ for 24 hours under anaerobic conditions.

\subsubsection{Plate Count Procedure Day 3}

On Day 3 , the agar plates of $B$. fragilis and $C$. perfringens were again examined for colony formation. Plates were still free and clear. Most likely the RCM plates contained dissolved oxygen content that inhibited growth for the anaerobic species. Pre-reduced agar can be purchased from a microbiology supplies company, however these plates were quite expensive and there was not adequate funding at the time to support the purchase. Therefore the plate count procedure was continued solely for the E. coli specimen.

Since the drug samples used in later experiments (Chapter 4) were dosed from an inoculated broth media, it was important to determine the approximate number of viable bacteria administered under experimental conditions. The $E$. coli test tube from Day 2 was removed from the chamber and then vortexed for 30 seconds. The optical 
density (OD) was recorded using a spectrophotometer set at $600 \mathrm{~nm}$. This served as a numerical value for sample turbidity and allowed the amount of bacterial growth between samples to be compared. Three of the remaining tubes of RCM broth were inoculated with $250 \mu \mathrm{L}$ of $E$. coli broth for replicate bacterial enumeration analysis. Samples were incubated at $37^{\circ} \mathrm{C}$ for 24 hours under anaerobic conditions. After the tubes were incubated, the supplies for the dilutions and plating for Day 4 were prepared. $9 \mathrm{~mL}$ of DI water was measured into 54 test tubes.

\subsubsection{Plate Count Procedure Day 4}

On Day 4, 3 sets of serial dilutions were prepared. Each inoculated test tube from Day 3 served as Tube \#1 for each Dilution Set. A test tube rack was set up with the culture tube in position \#1, followed by 9-9 mL tubes of sterile water labeled \#2 \#10. Tube \#1 was vortexed for 10 seconds and then a $1 \mathrm{~mL}$ aliquot from Tube \#1 was transferred by sterile pipet into Tube \#2. After the transfer, the tip was discarded and replaced with a new, sterile tip. This was done to ensure that there was no carryover between dilutions. In similar fashion, Tube \#2 was thoroughly mixed and then $1 \mathrm{~mL}$ of this solution was transferred into Tube \#3. This process was repeated until all of the dilutions were completed. This serial dilution method was applied to all 3 of the anaerobic E.coli cultures from Day 3.

A pour plate method was originally attempted in the viable cell plate counts. After making the dilution sets, $1 \mathrm{~mL}$ from each serial dilution tube was transferred to a sterile petri dish. Fresh RCM agar was autoclaved and then cooled to approximately $55^{\circ} \mathrm{C}$. The melted agar was poured over the top of the $1 \mathrm{~mL}$ aliquot of dilution sample. The dish was carefully swirled to encourage mixing and even dispersal of the aliquot 
within the agar. The plates were incubated at $37^{\circ} \mathrm{C}$ for 24 hours under anaerobic conditions.

There were several problems associated with this particular plate count method. As the agar cooled to the requisite $55^{\circ} \mathrm{C}$, the solution became clouded with solidifying agar aggregates. This created a problem as the plates were poured because large aggregations of agar would occasionally fall into the petri dishes. Not only did these solids prevent desirable mixing of the aliquot sample with the agar, they also caused some of the aliquot to be displaced out of the petri dish. This could affect the accuracy of the plate count method.

In addition, bubbles and foam formed within the agar solution as it cooled. These were transferred to pour plates along with the agar and made it difficult to distinguish viable cell growth from the set bubbles and foam. This too would have had an impact on the accuracy of the plate count. Finally, many bacteria display inhibited growth at higher temperature. The optimal growth of Escherichia coli occurs at $37^{\circ} \mathrm{C}$. Growth is significantly hindered at temperatures greater than $45^{\circ} \mathrm{C}$ because the elevated heat reduces the number of viable cells. Therefore, the raised agar temperature had the potential to kill some of the viable cells, which likewise would have affected the accuracy of the plate count. Thus, the spread plate method was chosen to be a better choice with regards to the accuracy of the plate count results.

To optimize the spread plate method, the amount of sample transferred onto the surface of the plate had to be determined. Aliquots of $1 \mathrm{~mL}$ and $100 \mu \mathrm{L}$ produced large landscapes of bacteria that coated the entire surface of the agar plate. No single colonies were observed, even after serial dilution. Smaller aliquots of $50 \mu \mathrm{L}, 25 \mu \mathrm{L}, 10$ 
$\mu \mathrm{L}, 5 \mu \mathrm{L}$, and $2.5 \mu \mathrm{L}$ were plated. While $50 \mu \mathrm{L}$ and $25 \mu \mathrm{L}$ still produced surface growth (lawn) over the entirety of the plate, a smattering of single colonies was observed with the $10 \mu \mathrm{L}, 5 \mu \mathrm{L}$, and $2.5 \mu \mathrm{L}$ aliquots. A new set of serial dilutions was performed and a $10 \mu \mathrm{L}$ aliquot of each was plated and incubated. Plates were counted using the Advanced QCount Automated Colony Counter. The majority of counts were within the 30-300 count required for accurate viable bacterial estimation. Therefore, the $10 \mu \mathrm{L}$ aliquot was chosen for future spread plating of $E$. coli.

The entire process was repeated from Day 1 to Day 4 for the E.coli sample under anaerobic as well as aerobic conditions for comparison. On Day 4, after the dilutions were prepared, the contents of Tube \#3 (102 dilution) were mixed thoroughly. A $10 \mu \mathrm{L}$ aliquot was transferred from Tube \#3 onto the surface of an RCM plate labeled $10^{2}$. The plate was placed on a petri dish turn table and the $10 \mu \mathrm{L}$ aliquot distributed using a plate spreader. This was done to ensure the agar surface was coated as evenly as possible. This method was applied to each of the remaining Tube \#4 - Tube\#10 and agar plates labeled $10^{3}$ through $10^{9}$ to complete Dilution Set \#1. This method was completed for the remaining dilution sets, and plates were incubated at $37^{\circ} \mathrm{C}$ for 24 hours under aerobic or anaerobic conditions. Figure 6 depicts the serial dilution procedure. 
Figure 6 - Serial Dilutions and Spread Plates

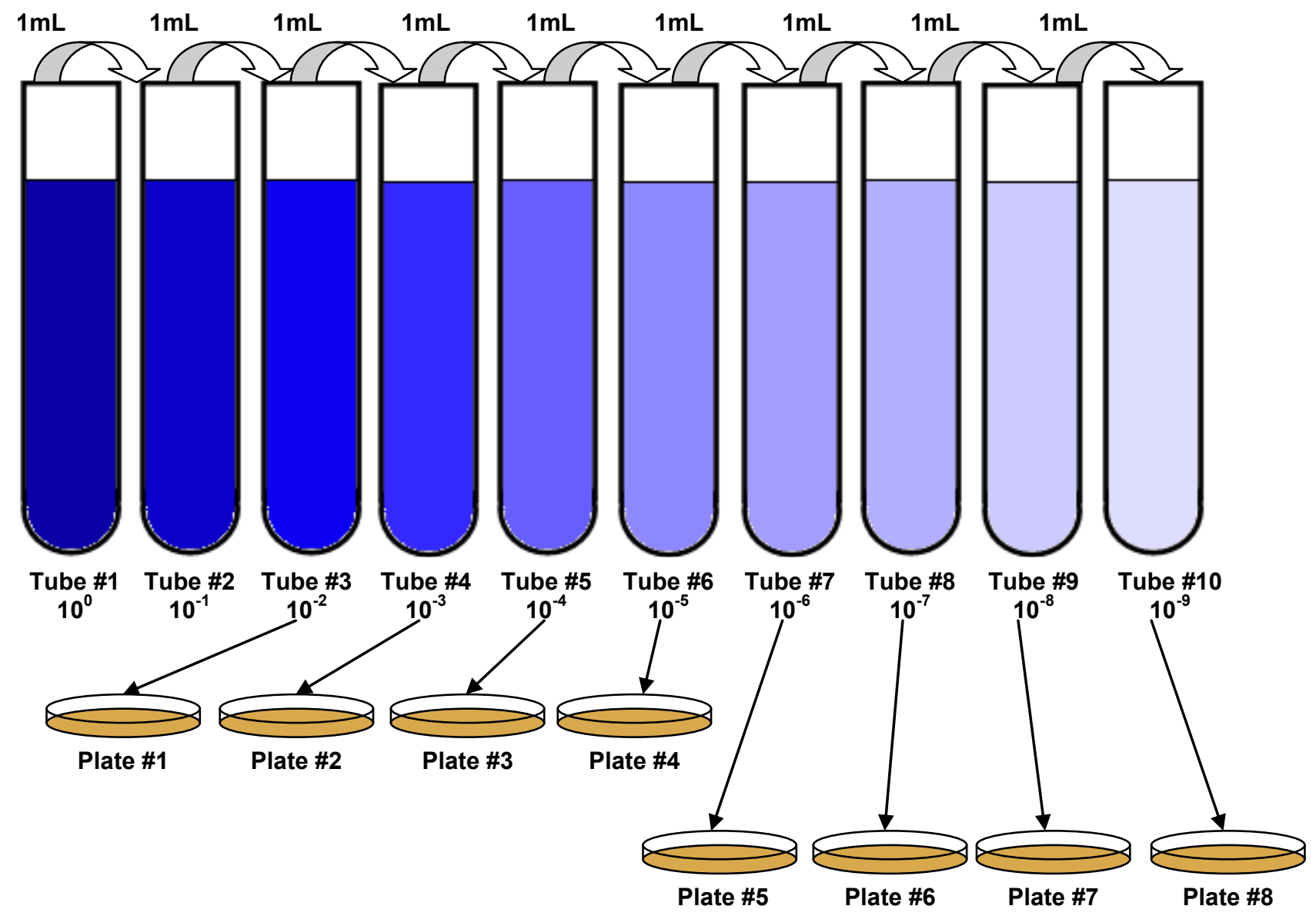

\subsubsection{Plate Count Procedure Day 5}

On Day 5, the colonies on the plates were counted using an Advanced QCount $\circledast$

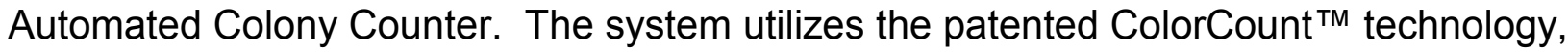
which is based on principles of color recognition. A lighted surface underneath the plate and adjustable background plates allow the user to achieve optimal resolution and achieve the most accurate plate count.

The plates were retrieved from their respective incubation chambers, and the QCount $\AA$ was used to count the colonies that formed on the surface of the plates. The 
plate was placed on the viewing surface agar-side down. The area multiplier was adjusted to $10 \%$ so as to include colonies that formed near the outer edges of the agar. Shutter speed was set to $1 / 250$, while the size of the colonies adjusted to $0.2-20 \mathrm{~mm}$.

Plates containing fewer than 30 colonies were considered too few to count (TFTC) while plates containing more than 300 colonies were considered too numerous to count (TNTC). The number of colonies was recorded for each plate using these specifications. Table 10 summarized the plate count results for $E$. coli under aerobic conditions in RCM while Table 11 summarized results under anaerobic conditions.

Table 10 - Aerobic Plate Count for E. coli

\begin{tabular}{|cccc|}
\hline Dilution Factor & Set \#1 & Set \#2 & Set\#3 \\
\hline $\mathbf{1 0}^{\mathbf{2}}$ & TNTC & TNTC & TNTC \\
\hline $\mathbf{1 0}^{\mathbf{3}}$ & TNTC & TNTC & TNTC \\
\hline $\mathbf{1 0}^{4}$ & TNTC & TNTC & TNTC \\
\hline $\mathbf{1 0}^{\mathbf{5}}$ & 63 & 143 & 96 \\
\hline $\mathbf{1 0}^{\mathbf{6}}$ & 34 & 29 & 69 \\
\hline $\mathbf{1 0}^{\mathbf{7}}$ & TFTC & TFTC & TFTC \\
\hline $\mathbf{1 0}^{\mathbf{8}}$ & TFTC & TFTC & TFTC \\
\hline $\mathbf{1 0}^{\mathbf{9}}$ & TFTC & TFTC & TFTC \\
\hline
\end{tabular}

Table 11 - Anaerobic Plate Count for E. coli

\begin{tabular}{|cccc|}
\hline Dilution Factor & Set \#1 & Set \#2 & Set\#3 \\
\hline $\mathbf{1 0}^{\mathbf{2}}$ & TNTC & TNTC & TNTC \\
\hline $\mathbf{1 0}^{\mathbf{3}}$ & TNTC & TNTC & TNTC \\
\hline $\mathbf{1 0}^{\mathbf{4}}$ & 58 & 41 & 31 \\
\hline $\mathbf{1 0}^{\mathbf{5}}$ & 39 & 35 & 33 \\
\hline $\mathbf{1 0}^{\mathbf{6}}$ & TFTC & TFTC & TFTC \\
\hline $\mathbf{1 0}^{\mathbf{7}}$ & TFTC & TFTC & TFTC \\
\hline $\mathbf{1 0}^{\mathbf{8}}$ & TFTC & TFTC & TFTC \\
\hline $\mathbf{1 0}^{\mathbf{9}}$ & TFTC & TFTC & TFTC \\
\hline
\end{tabular}


To calculate the approximate number of colony forming units (CFUs) in each sample, the appropriate dilution factor was applied to the colony number count. In Anaerobic Plate Count for E. coli the $10^{4}$ plate contained 58 colonies. The calculation was performed using Equation 8.

\# Colonies $=58$

Dilution Factor $=10^{4}$

Volume of Dilution $=0.010 \mathrm{~mL}$

Equation 8 - Determination of Colony Forming Units

$$
\text { CFUs }=\frac{\# \text { Colonies } \times \text { Dilution Factor }}{\text { Volume of Dilution }}=\frac{\text { CFU }}{\mathrm{mL}}
$$

Therefore, in this particular example,

$$
\text { CFUs }=\frac{58 \times 10^{4}}{0.010 \mathrm{~mL}}=\frac{58 \times 10^{6} \mathrm{CFU}}{\mathrm{mL}}=5.8 \times 10^{7} \mathrm{CFU} / \mathrm{mL}
$$

Only plates that contained between $30-300$ colonies were included in the analysis. The aerobic CFU/mL data was reported in Table 12, and anaerobic CFU/mL data in Table 13.

Table 12 - Average Aerobic CFU/mL for E. coli

\begin{tabular}{|cccc|}
\hline & & & \\
Dilution Factor & \multicolumn{3}{c|}{ Individual Plate Counts (CFU/mL) } \\
\hline $\mathbf{1 0}^{5}$ & $6.3 \times 10^{8}$ & $1.4 \times 10^{9}$ & $9.6 \times 10^{8}$ \\
\hline $\mathbf{1 0}^{6}$ & $3.4 \times 10^{9}$ & $2.9 \times 10^{9}$ & $6.9 \times 10^{9}$ \\
\hline Set Average & $2.0 \times 10^{9}$ & $2.2 \times 10^{9}$ & $3.9 \times 10^{9}$ \\
\hline Overall Average & $2.7 \times 10^{9}$ & & \\
\hline
\end{tabular}


Table 13 - Average Anaerobic CFU/mL for E.coli

\begin{tabular}{|clll|}
\hline Dilution & \multicolumn{3}{c|}{ Individual Plate Counts (CFU/mL) } \\
\hline $10^{4}$ & $5.8 \times 10^{7}$ & $4.1 \times 10^{7}$ & $3.1 \times 10^{7}$ \\
\hline $10^{5}$ & $3.9 \times 10^{8}$ & $3.5 \times 10^{8}$ & $3.3 \times 10^{8}$ \\
\hline Set Average & $2.2 \times 10^{8}$ & $2.0 \times 10^{8}$ & $1.8 \times 10^{8}$ \\
\hline Overall Average & $2.0 \times 10^{8}$ & & \\
\hline
\end{tabular}

As expected, the $\mathrm{CFU} / \mathrm{mL}$ was greater for the aerobic preparations than the anaerobic preparations. This was due to the fact that $E$. coli, a facultative anaerobic species, grew best under aerobic conditions (average $\mathrm{OD}=0.770$ ), but was also capable of growth under limited oxygen or anaerobic conditions to a lesser extent (average $\mathrm{OD}=0.664$ ). These $\mathrm{CFU} / \mathrm{mL}$ counts were near the approximate ranges of viable populations found in the human digestive tract as reported by the $\mathrm{NIH}$, specifically the ileum $\left(10^{5}-10^{8}\right)$ and colon $\left(10^{10}-10^{12}\right){ }^{45}$

\subsubsection{Cellular Lysis}

\subsubsection{Chemical Lysis}

Several methods of cellular lysis were considered during method development. Bug Buster ${ }^{\circledR}$ Protein Extraction Reagent by Novagen ${ }^{\circledR}$ is a type of chemical lysis solution that chemically disrupts the cellular structure of $E$. coli to release proteins. The solution consists of a mixture of Tris-buffer and detergents that perforate the cell wall. This type of lysis was eliminated because RCM is a complex matrix, and addition of the lysis solution would further complicate the extraction and analysis of the target compounds and their metabolites. 


\subsubsection{Microfluidizer}

The microfluidizer is a type of homogenization instrument used to perform mechanical lysis. The sample is driven into the interaction chamber where it encounters a series of microchannels. High velocity flow of the sample creates high shear which acts in combination with impact forces to disrupt the cellular structure.

A simple study was conducted using a Microfluidics M-110P to determine potential effects of microfluidization on sample analysis. This instrument is depicted in Figure 7.

Figure 7 - Microfluidics M-110P Microfluidizer

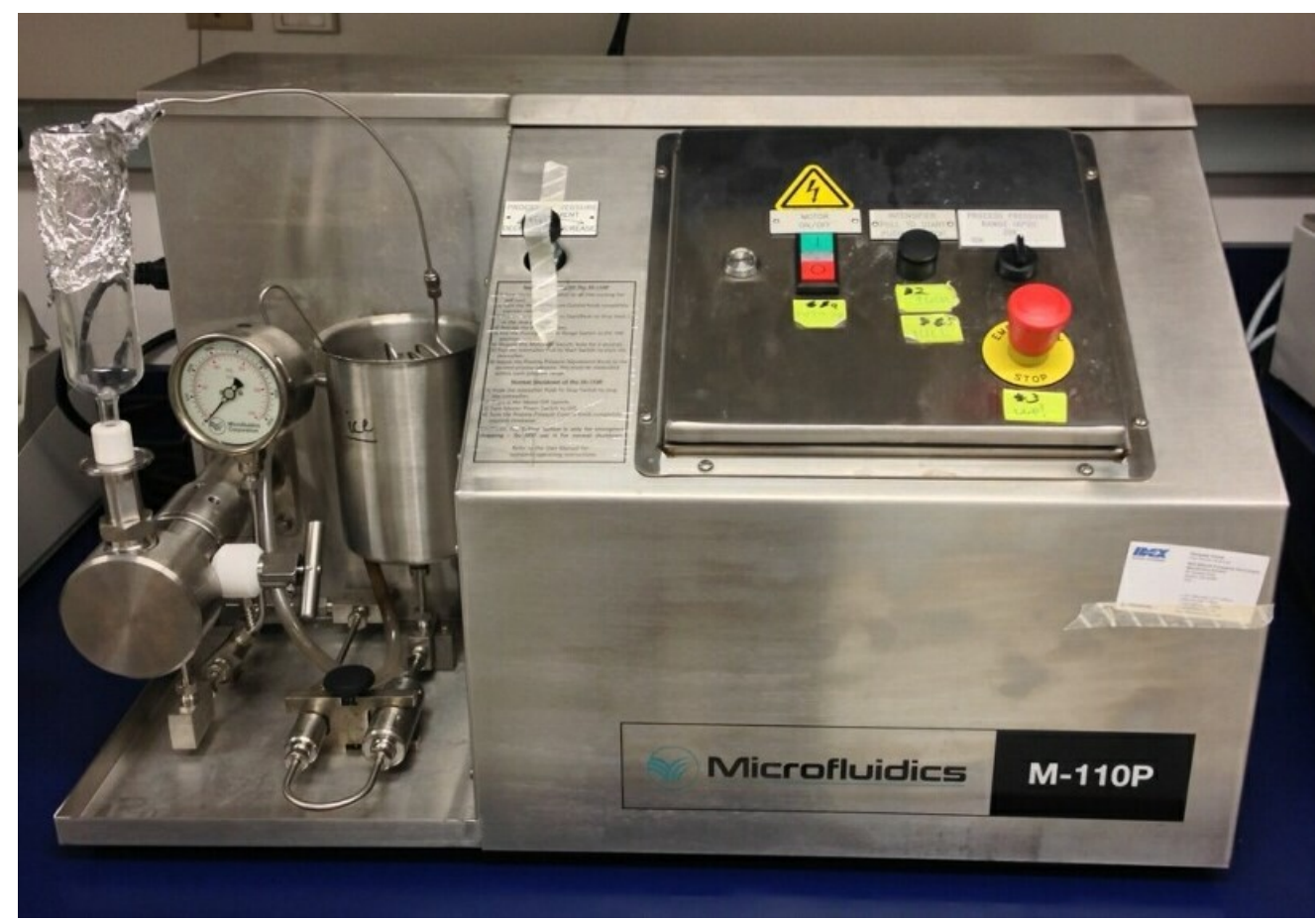

A vial with lid was weighed empty, and then weighed again after addition of $5 \mathrm{~mL}$ of water. The water sample in each vial was passed through the microfluidizer three times, and mass recorded after each pass. The purpose of this experiment was to determine whether the instrumental design of the microfluidizer would have an effect on 
sample retrieval from the system. The change in mass between runs was designed to identify changes in sample volume. The microfluidizer data is summarized in Table 14.

Table 14 - Effects of the Microfluidizer on Sample Volume

\begin{tabular}{|cccccc|}
\hline Tube \# & Empty Vial + Lid $(\mathbf{g})$ & $\mathbf{+} \mathbf{5} \mathbf{m L ~} \mathbf{D I ~ H}_{\mathbf{2}} \mathbf{O}$ & Micro1 $\mathbf{( g )}$ & Micro2 $(\mathbf{g})$ & Micro3 $(\mathbf{g})$ \\
\hline $\mathbf{1}$ & 6.40 & 11.33 & 11.38 & 11.32 & 11.26 \\
\hline $\mathbf{2}$ & 6.38 & 11.27 & 11.19 & 11.23 & 11.20 \\
\hline $\mathbf{3}$ & 6.40 & 11.35 & 11.48 & 11.43 & 11.49 \\
\hline $\mathbf{4}$ & 6.51 & 11.50 & 11.42 & 11.40 & 11.40 \\
\hline $\mathbf{5}$ & 6.41 & 11.30 & 11.20 & 10.88 & 11.04 \\
\hline $\mathbf{6}$ & 6.41 & 11.25 & 11.20 & 11.14 & 11.08 \\
\hline $\mathbf{7}$ & 6.41 & 11.36 & 11.39 & 11.44 & 11.29 \\
\hline $\mathbf{8}$ & 6.39 & 11.32 & 11.40 & 11.39 & 11.44 \\
\hline $\mathbf{9}$ & 6.41 & 11.37 & 11.34 & 11.23 & 11.15 \\
\hline $\mathbf{1 0}$ & 6.43 & 11.38 & 11.51 & 11.56 & 11.45 \\
\hline
\end{tabular}

Averages of the microfluidizer analyses were taken and then subtracted from the mass of vial, lid, and $5 \mathrm{~mL}$ of water. The average volume difference was determined to be approximately $0.03 \mathrm{~g} \pm 0.13 \mathrm{~g}$. These results are detailed in Table 15. At room temperature, the density of water is approximately $1 \mathrm{~g} / \mathrm{mL}$. Sample lysis by microfluidizer therefore resulted in an average of $30 \mu \mathrm{L}( \pm 130 \mu \mathrm{L})$ increase in sample volume. This would have affected the concentration of samples prior to LC-MS/MS analysis, which would have provided for inaccurate quantitation. In addition, there was no way to guarantee proper flushing of the system between samples which could lead to analyte carryover and sample contamination issues as well. 
Table 15 - Volume Differences after Microfluidizer Analysis

\begin{tabular}{|cccc|}
\hline Tube \# & $\mathbf{5 m L ~ D I ~}_{\mathbf{2}} \mathbf{O} \mathbf{( g )}$ & Micro Average $\mathbf{( g )}$ & Difference $\mathbf{( g )}$ \\
\hline $\mathbf{1}$ & 4.93 & 4.92 & +0.01 \\
\hline $\mathbf{2}$ & 4.89 & 4.83 & +0.06 \\
\hline $\mathbf{3}$ & 4.95 & 5.07 & -0.12 \\
\hline $\mathbf{4}$ & 4.99 & 4.90 & +0.09 \\
\hline $\mathbf{5}$ & 4.89 & 4.63 & +0.26 \\
\hline $\mathbf{6}$ & 4.84 & 4.73 & +0.11 \\
\hline $\mathbf{7}$ & 4.95 & 4.96 & -0.01 \\
\hline $\mathbf{8}$ & 4.93 & 5.02 & -0.09 \\
\hline $\mathbf{9}$ & 4.96 & 4.83 & +0.13 \\
\hline $\mathbf{1 0}$ & 4.95 & 5.08 & -0.13 \\
\hline
\end{tabular}

\subsubsection{Sonication}

Sonication is another technique used in cellular lysis. Three samples containing the benzodiazepine compounds and metabolites were prepared at $100 \mathrm{ng} / \mathrm{mL}$ and quantified via LC-MS/MS. After analysis, the samples were sonicated for 10 minutes and then reanalyzed. Post-sonication samples exhibited a concentration decrease for all analytes and metabolites, ranging from -3.6 to $-6.2 \mathrm{ng} / \mathrm{mL}$. Because of the extent of concentration decrease, sonication was likewise ruled out as a method of cellular lysis for this research. Analysis was performed directly on bacterial samples without cellular disruption or lysis. 


\section{Chapter 3: Method Validation - Simultaneous Quantitation of Diazepam, Flunitrazepam, and Metabolites in Reinforced Clostridial Medium by Liquid Chromatography-Tandem Mass Spectrometry}

\subsubsection{Introduction}

Analytical and forensic toxicology revolve around the detection, identification, and quantitation of compounds, particularly alcohol, pharmaceuticals, drugs of abuse, and other toxic or lethal substances. There are many benzodiazepine methods available for common matrices such as blood ${ }^{46-48}$, plasma ${ }^{49}$, hair ${ }^{50,51}$, and urine ${ }^{51-53}$, but these matrices do not provide all of the nutrients required to support the development and proliferation of bacterial species. A medium like RCM, which was discussed in Table 2, however, provides the nutrients required to promote bacterial growth and therefore metabolism. Diazepam (DZ) and flunitrazepam (FLU) were chosen as the analytes of interest because the human metabolites have been previously determined and chemical standards were commercially available.

As detailed in Chapter 1, several studies have demonstrated that bacteria are capable of metabolizing complex chemical substances including drugs of abuse. But, before the metabolic effects of GI bacteria on DZ and FLU could be investigated and compared to those of human metabolism, a method for the analysis and quantitation of these benzodiazepines and their metabolites in RCM needed to be developed. To perform these tasks with reliability and certainty, the method likewise needed to be validated. 
The presented method includes development and validation of a novel LCMS/MS method for the detection, identification, and quantitation of DZ, FLU, and their respective metabolites in RCM. Application of this method was discussed in Chapter 4.

$\mathrm{DZ}$ is classified as a Schedule IV benzodiazepine by the DEA, and acts as a central nervous system (CNS) depressant. ${ }^{54}$ In the medical industry it is most commonly known as Valium ${ }^{\circledR}$ and is usually administered in pill form, as a gel, or as an injection. Street names include yellow V's, blue V's, foofoo's, and Howards.

It is most frequently prescribed as a sedative or tranquilizer, but can also be used in treatment of anxiety, convulsive disorders, and muscle spasms. Health effects include drowsiness, vertigo, vomiting, depressed respiration, elevated heart rate, coma, and death, particularly when the drug is abused. ${ }^{55}$ Known human metabolites of DZ include nordiazepam (NDZ), oxazepam (OX), and temazepam (TZ). ${ }^{46,48,50-52,56}$

FLU is also classified as a benzodiazepine and central nervous system depressant. The DEA classifies it as a Schedule IV drug of abuse, however, possession and distribution are considered Schedule I offenses. The manufacture, importation, sale, and usage are illegal in the United States. ${ }^{24,54,56}$

By trade, FLU is labeled as Rohypnol ${ }^{\circledR}$, and is commonly abused as a "date rape" drug. On the street it may be referred to by a number of nicknames including circles, forget-me-pill, La Rocha, lunch money, Mexican Valium, Reynolds, roach, roofies, and wolfies to name just a few. ${ }^{24}$

FLU can be taken in pill form, or crushed to be either snorted or ingested. The powder is colorless, odorless, and tasteless when mixed into beverages and food, making it difficult to detect. Upon administration, it acts to incapacitate the victim and 
prevent resistance, often in cases of sexual assault. Health effects include drowsiness, decreased anxiety, and impaired mental function. Excessive doses can result in unconsciousness, anterograde amnesia, respiration suppression, and death ${ }^{24}$ Human metabolites of FLU metabolites include 7-aminoflunitrazepam (7AM) and $\mathrm{N}$ desmethylflunitrazepam (NDES). 47,48,51,53,57-59 47, 57,48, 51,53,58, 59

\subsubsection{Materials and Methods}

\subsubsection{Materials}

Reference standards for DZ, NDZ, OX, TZ, FLU, 7AM, and NDES as $1.0 \mathrm{mg} / \mathrm{mL}$ and diazepam-d5 (DZ-d5), nordiazepam-d5 (NDZ-d5), oxazepam-d5 (OX-d5), temazepam-d5 (TZ-d5), flunitrazepam-d7 (FLU-d7), 7-aminoflunitrazepam-d7 (7AM-d7), and N-desmethylflunitrazepam-d4 (NDES-d4) as $100 \mu \mathrm{g} / \mathrm{mL}$ were purchased from Cerilliant ${ }^{\circledR}$ (Round Rock, TX). Reagents and solvents consisted of 2-propanol (IPA) (Optima ${ }^{\text {TM }}$ LC/MS) purchased from Fischer Scientific (Pittsburgh, PA), HPLC grade ethyl acetate (EA) from JT Baker® (Center Valley, PA), and formic acid (FA) eluent additive for LC-MS and LC-MS CHROMASOLV® acetonitrile (ACN) and methanol $(\mathrm{MeOH})$ from Sigma-Aldrich® (St. Louis, MO). The Strata-X-Drug N 100 $\mu$ Polymer RP $60 \mathrm{mg} / 3 \mathrm{~mL}$ cartridges used in sample preparation were obtained from Phenomenex® (Torrance, CA). Compounds were separated using a narrow bore ZORBAX Eclipse XDB-C8 $(2.1 \mathrm{~mm} \times 150 \mathrm{~mm} \times 5 \mu \mathrm{m})$ column affixed with a ZORBAX Eclipse XDB-C8 (2.1 $\mathrm{mm} \times 12.5 \mathrm{~mm} \times 5 \mu \mathrm{m})$ guard cartridge from Agilent Technologies (Santa Clara, CA). RCM was obtained from BD Difco ${ }^{\mathrm{TM}}$ (Sparks, MD). 


\subsubsection{Sample Preparation}

Several working solutions were used to prepare the dilution samples required for method validation analyses. All working solutions were prepared in water/methanol $(60 / 40 \mathrm{v} / \mathrm{v})$. An internal standard working solution consisting of the seven deuterated analytes was prepared at $5000 \mathrm{ng} / \mathrm{mL}$, and an analyte working standard consisting of the seven analytes of interest at $10,000 \mathrm{ng} / \mathrm{mL}$ (WS1). Two additional working standards were prepared by dilution of WS1 at $1000 \mathrm{ng} / \mathrm{mL}$ (WS2), and $10 \mathrm{ng} / \mathrm{mL}$ (WS3).

The matrix consisted of ten $100 \mathrm{~mL}$ aliquots of RCM $(3.8 \mathrm{~g} \mathrm{RCM}$ in $100 \mathrm{~mL}$ of DI water) that were pooled and then autoclaved at $121^{\circ} \mathrm{C}$ for 15 minutes. Calibrators and sample dilutions were prepared in RCM from the appropriate working standard. The final internal standard concentration in all $1 \mathrm{~mL}$ samples was $50 \mathrm{ng} / \mathrm{mL}$.

\subsubsection{Sample Extraction}

An initial calibration curve was prepared from working solutions in buffer and plotted at $0.01,0.05,0.1,0.5,1,5,10,50,100,250$, and $500 \mathrm{ng} / \mathrm{mL}$. Calibration curves for each analyte in buffer can be seen in Appendix A. Four different SPE methods were evaluated for recovery of DZ, FLU, and metabolites. Reagents and solvents consisted of acetonitrile (ACN), ethyl acetate (EA), isopropyl alcohol (IPA), formic acid (FA), methanol $(\mathrm{MeOH})$, and ammonium hydroxide $\left(\mathrm{NH}_{4} \mathrm{OH}\right)$. Details of the methods are shown in Figures 8 and 9.

Samples were extracted from RCM at three concentrations $0.05,0.5$, and 100 $\mathrm{ng} / \mathrm{mL}$ and then analyzed via LC-MS/MS. Results were evaluated based upon the 
retention time, accuracy, and ion ratios of analytes after extraction. SPE method 1 was determined to be the best choice for extraction of all of the analytes of interest.

Figure 8 - SPE Method 1\&2 for Diazepam, Flunitrazepam, and Metabolites

\begin{tabular}{|c|c|c|}
\hline Cartr & $\begin{array}{c}\text { SPE } 1 \\
\text { dge: Strata-X-Drug N }\end{array}$ & $\begin{array}{c}\text { SPE } 2 \\
\text { Cartridge: Strata-X-Drug B }\end{array}$ \\
\hline Wash & - $\mathrm{ACN} / \mathrm{H}_{2} \mathrm{O}(20 / 80 \mathrm{v} / \mathrm{v})$ & $\begin{array}{ll} & \cdot 0.1 \% \mathrm{FA} \\
\text { Wash } & \cdot \mathrm{MeOH} / \mathrm{H}_{2} \mathrm{O}(30 / 70 \mathrm{v} / \mathrm{v})\end{array}$ \\
\hline & - 10 minutes & - 10 minutes \\
\hline Elute & -EA/IPA (85/15 v/v) & $\begin{array}{l}\cdot 2 \% \mathrm{NH}_{4} \mathrm{OH} \text { in } \\
\mathrm{ACN} / \mathrm{MeOH}(1 / 1 \mathrm{v} / \mathrm{v})\end{array}$ \\
\hline
\end{tabular}

Figure 9 - SPE Method 3\&4 for Diazepam, Flunitrazepam, and Metabolites

\begin{tabular}{|c|c|c|}
\hline Cartr & $\begin{array}{c}\text { SPE } 3 \\
\text { dge: Strata-X-Drug N }\end{array}$ & $\begin{array}{c}\text { SPE } 4 \\
\text { Cartridge: Strata-X-Drug b }\end{array}$ \\
\hline Wash & $\begin{array}{l}\cdot \mathrm{H}_{2} \mathrm{O} \\
\cdot 20 \% \mathrm{MeOH}\end{array}$ & $\begin{array}{ll}\cdot \mathrm{H}_{2} \mathrm{O} \\
\text { Wash } & \cdot 15 \% \mathrm{MeOH}\end{array}$ \\
\hline Dry & -10 minutes & -10 minutes \\
\hline Elute & $\cdot 100 \% \mathrm{MeOH}$ & $\cdot 100 \% \mathrm{MeOH}$ \\
\hline
\end{tabular}


Samples were extracted from RCM prior to analysis to minimize salts and other compounds that could interfere with or inhibit LC-MS/MS analysis. The Strata-X-Drug N cartridge is a polymeric reversed phase cartridge designed for neutral drugs of abuse such as benzodiazepine compounds. Solid phase extraction was performed on a vacuum manifold which can be seen in Figure 10.

Figure 10 - Solid Phase Extraction Vacuum Manifold

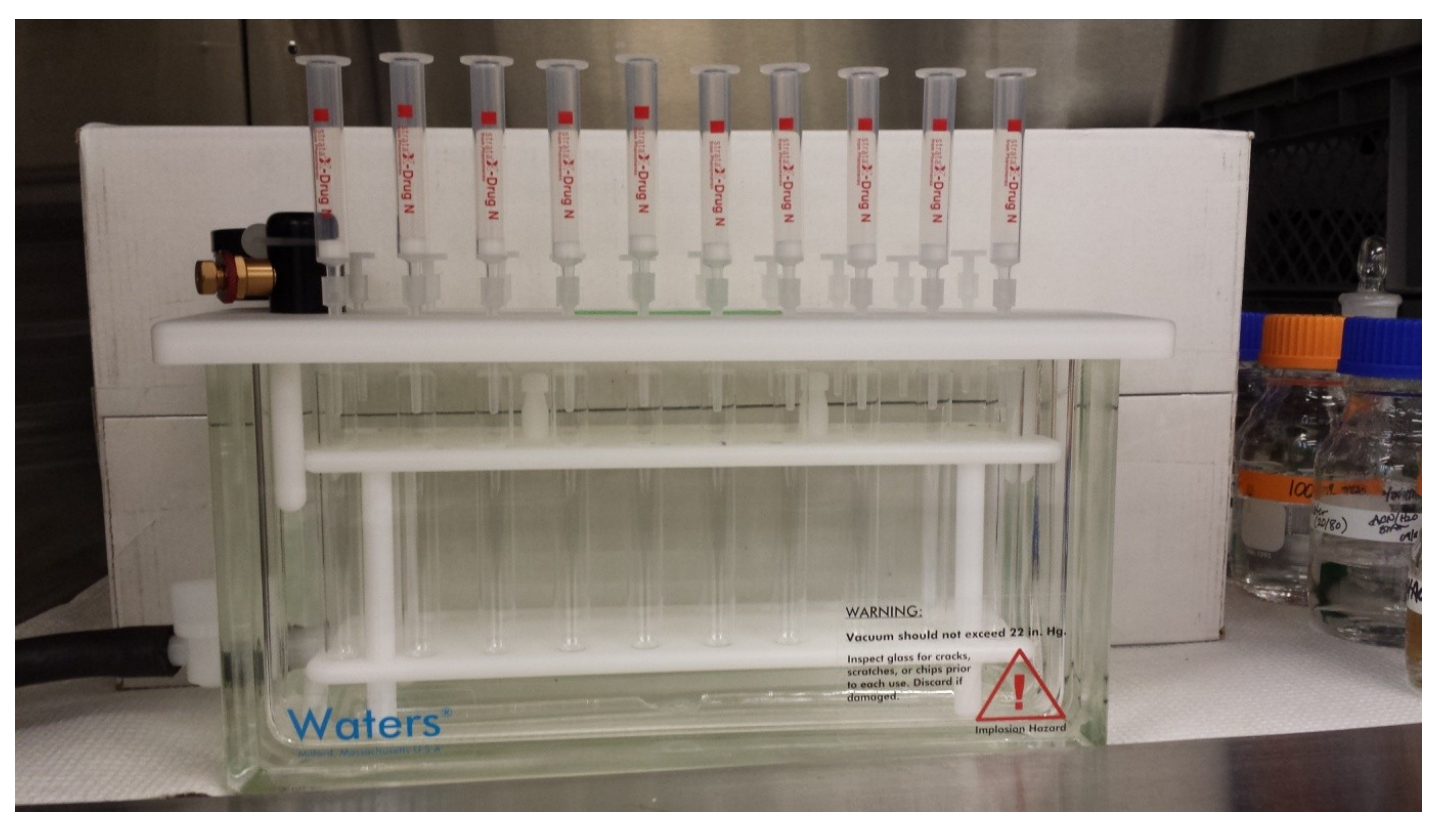

The manifold allowed for controlled extraction of up to 20 samples at a time. Following extraction, samples were dried on another manifold under ultrapure nitrogen supplied by a generator. The drying manifold apparatus is depicted in Figure 11 . 
Figure 11 - SPE Nitrogen Dry Down Manifold

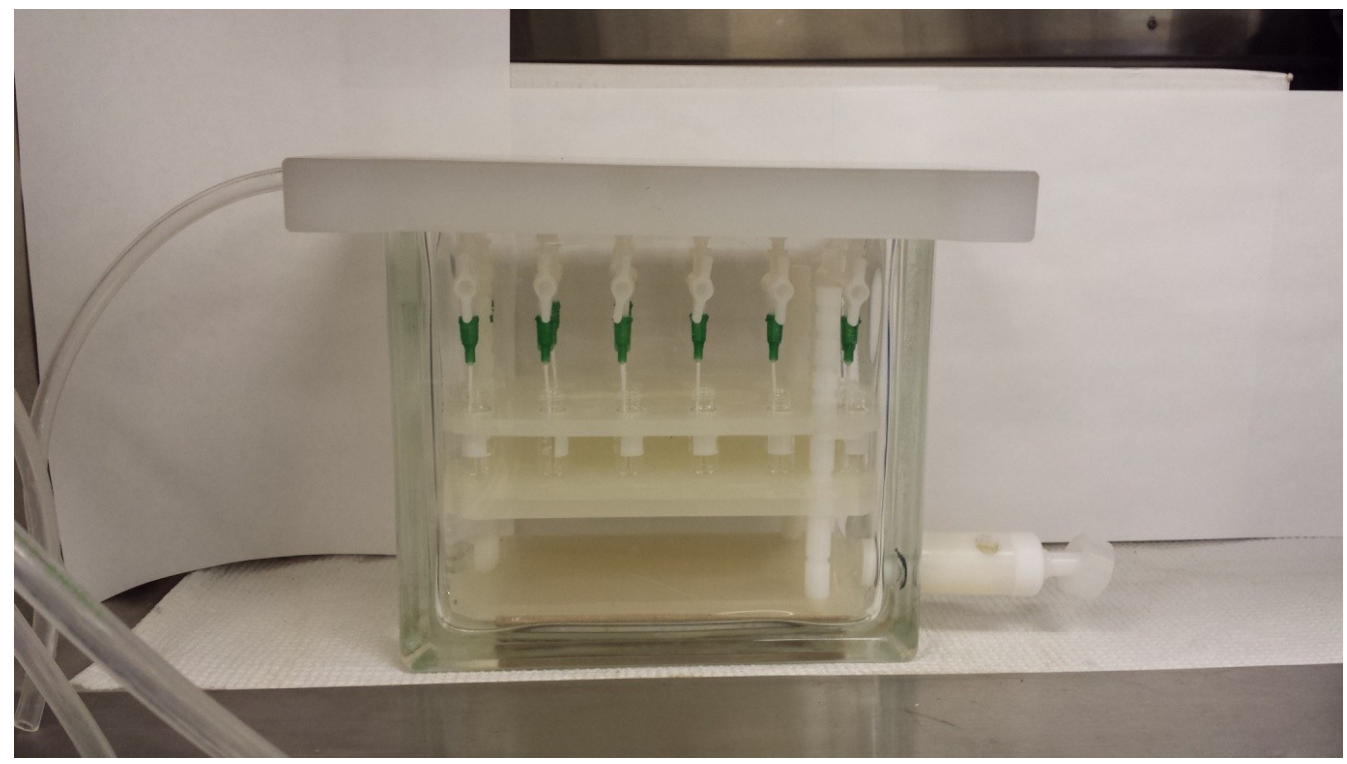

\subsubsection{Instrumental Analysis}

Analysis was performed using an Agilent G6490 Triple Quadrupole LC/MS system equipped with iFunnel, G4226A HiP autosampler, G4220A binary pump, and G1316C column compartment. Instrumentation can be seen in Figure 12. 
Figure 12 - Agilent G6490 Triple Quadrupole LC/MS

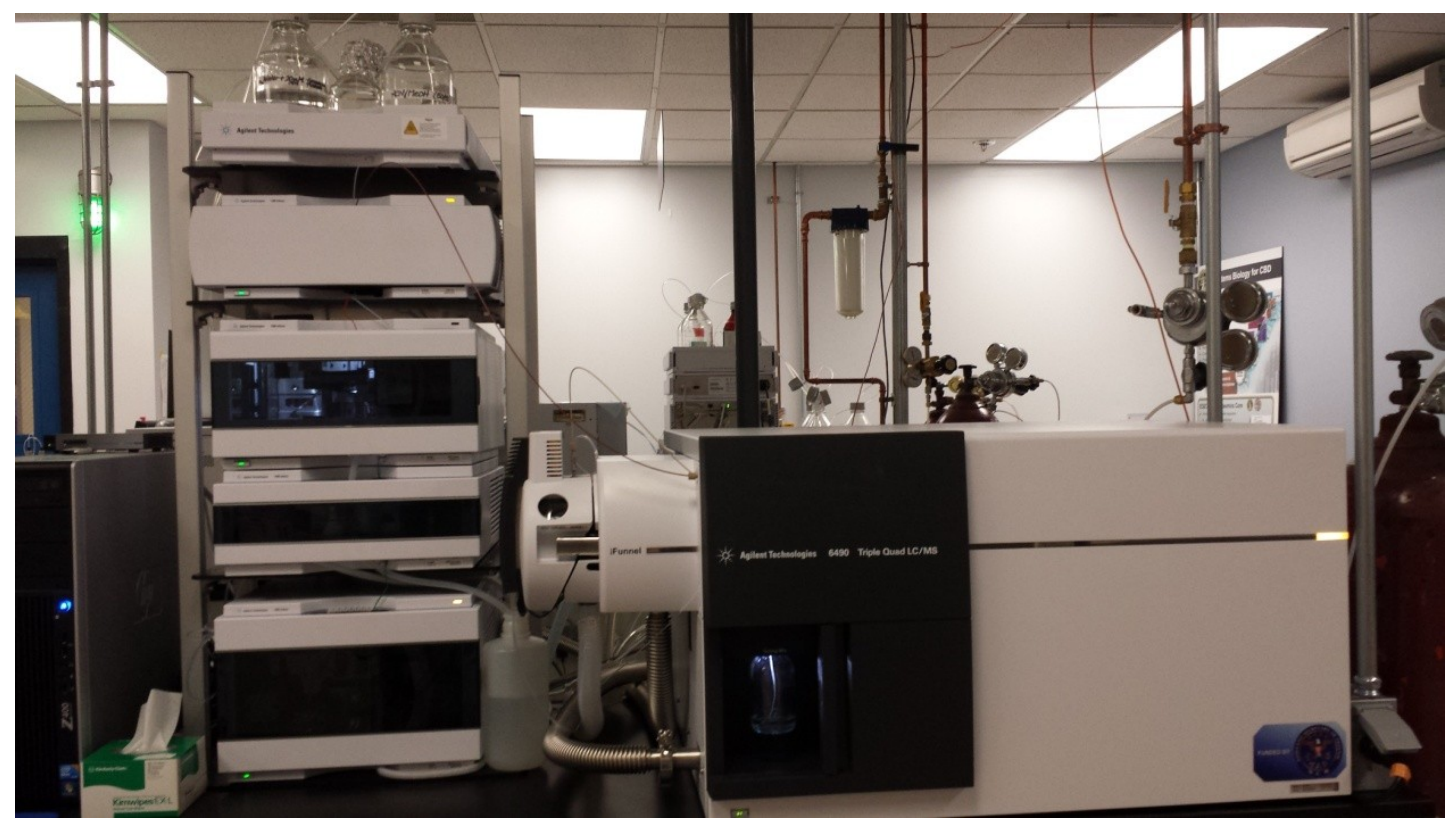

$2 \mu \mathrm{L}$ of sample was injected into the system at a flow rate of $0.250 \mathrm{~mL} /$ minute.

Mobile phases of water with $0.1 \%$ formic acid $(\mathrm{A})$ and acetonitrile with $0.1 \%$ formic acid (B) were used in a gradient elution program of $40 \%$ B to $70 \%$ B over 6 minutes, holding at $70 \%$ B for 0.5 minutes, returning to original $40 \%$ B conditions over 0.1 minutes, and then a column equilibration of 1.4 minutes for a total run time of 8 minutes. A post-run time of 2 minutes at $40 \%$ B was included between runs to ensure column equilibration before the next injection.

Samples were ionized via Agilent Jet Stream electrospray ionization source (AJS ESI) in the positive mode. Parameters were optimized with the Agilent MassHunter ${ }^{\mathrm{TM}}$ Source and iFunnel Optimizer software using a $100 \mathrm{ng} / \mathrm{mL}$ extracted sample of DZ, FLU, and their respective metabolites. The range of values explored for each parameter, the increment by which conditions were changed, and the final optimized value are summarized in Table 16. Other source parameters included the $\triangle \mathrm{EMV}(400)$, 
fragmentor $(380 \mathrm{~V})$, and cell acceleration $(2 \mathrm{~V})$. Column temperature was maintained at $37^{\circ} \mathrm{C}$ and autosampler at $20^{\circ} \mathrm{C}$.

Table 16 - Source and iFunnel Optimization for Diazepam, Flunitrazepam, and Respective Metabolites in RCM

\begin{tabular}{|cccc|}
\hline Parameter & Range & Increment & Optimized \\
\hline Capillary Voltage (V) & $500-6000$ & 200 & 3250 \\
\hline Gas Temperature $\left({ }^{\circ} \mathbf{C}\right)$ & $120-290$ & $20^{\circ} \mathrm{C}$ & 265 \\
\hline Gas Flow (L/min) & $11-20$ & 2 & 19 \\
\hline Nebulizer (psi) & $15-60$ & 5 & 50 \\
\hline Sheath Gas Temperature $\left({ }^{\circ} \mathbf{C}\right)$ & $150-400$ & $20^{\circ} \mathrm{C}$ & 350 \\
\hline Sheath Gas Flow (L/min) & $10-12$ & 1 & 11 \\
\hline
\end{tabular}

MassHunter ${ }^{\mathrm{TM}}$ Optimizer software was used to determine the best multiple reaction monitoring (MRM) transitions for each analyte of interest as well as their corresponding deuterated standards. The software provided up to four ion transitions for each target analytes and metabolite compound. These tables can be seen in Appendix B. All of the suggested ions were included in initial calibration. However, the final quantifier and qualifier ions chosen for the method were those that demonstrated the greatest signal response while also maintaining stable ion ratio across the calibration range.

MRM data was only acquired in a narrow window based upon the retention time of the analyte of interest. Because some of the ions had similar ion transitions, the narrow range of acquisition increased the selectivity for each individual analytes. Samples were quantified by comparing the peak area response ratio of the target analyte to that of the corresponding deuterated standard. The retention time (RT), 
quantifier and (qualifier) MRM transitions, collision energy (CE), and ion ratio for each set of analytes and corresponding deuterated standards is summarized in Table 17.

Table 17 - LC-MS/MS Parameters for Diazepam, Flunitrazepam, and Metabolites in $\mathrm{RCM}$

\begin{tabular}{|cccccc|}
\hline Analyte & $\begin{array}{c}\text { RT } \\
(\mathbf{m i n s})\end{array}$ & $\begin{array}{c}\text { Q1 } \\
(\mathbf{m} / \mathbf{z})\end{array}$ & $\begin{array}{c}\text { Q3 } \\
(\mathbf{m} / \mathbf{z})\end{array}$ & $\begin{array}{c}\text { CE } \\
(\mathbf{V})\end{array}$ & $\begin{array}{c}\text { Ion } \\
\text { Ratio }\end{array}$ \\
\hline DZ & 5.13 & 285.1 & 153.9 & 25 & 66.3 \\
\hline DZ-d5 & & & $(222.1)$ & 25 & \\
\hline NDZ & 3.92 & 271.1 & 140.1 & 21 & 69.2 \\
\hline & & & $(208.1)$ & 25 & \\
\hline NDZ-d5 & & 276.1 & 213.1 & 25 & \\
\hline OX & 3.58 & 287.1 & 241.1 & 17 & 16.3 \\
\hline & & & $(162.9)$ & 37 & \\
\hline OX-d5 & & 292.1 & 246.1 & 17 & \\
\hline TZ & 4.52 & 301.1 & 255.1 & 17 & 12.5 \\
\hline & & & $(176.9)$ & 37 & \\
\hline TZ-d5 & & 306.1 & 260.1 & 17 & \\
\hline FLU & 4.51 & 314.1 & 268.1 & 21 & 46.4 \\
\hline & & & $(239.1)$ & 33 & \\
\hline FLU-d7 & & 322.2 & 276.1 & 25 & \\
\hline 7AM & 2.08 & 284.1 & 135.1 & 25 & 36.1 \\
\hline & & & $(226.2)$ & 29 & \\
\hline 7AM-d7 & & 291.2 & 138.0 & 25 & \\
\hline NDES & 3.69 & 300.1 & 253.9 & 21 & 43.7 \\
\hline & & & $(197.9)$ & 37 & \\
\hline NDES-d4 & & 304.1 & 258.0 & 21 & \\
\hline
\end{tabular}

\subsubsection{Method Validation}

Definitions and guidelines used in method validation were detailed in section 1.5.0. A full validation including a calibration model as well as interference studies, carryover effects, ionization suppression/ enhancement, recovery, bias, precision, limit of detection (LOD), limit of quantitation (LOQ), dilution integrity, stability, and robustness 
of the method were evaluated. The MRM output for the method was depicted in Figure 13.

Figure 13 - MRM of Diazepam, Flunitrazepam, and Respective Metabolites in RCM

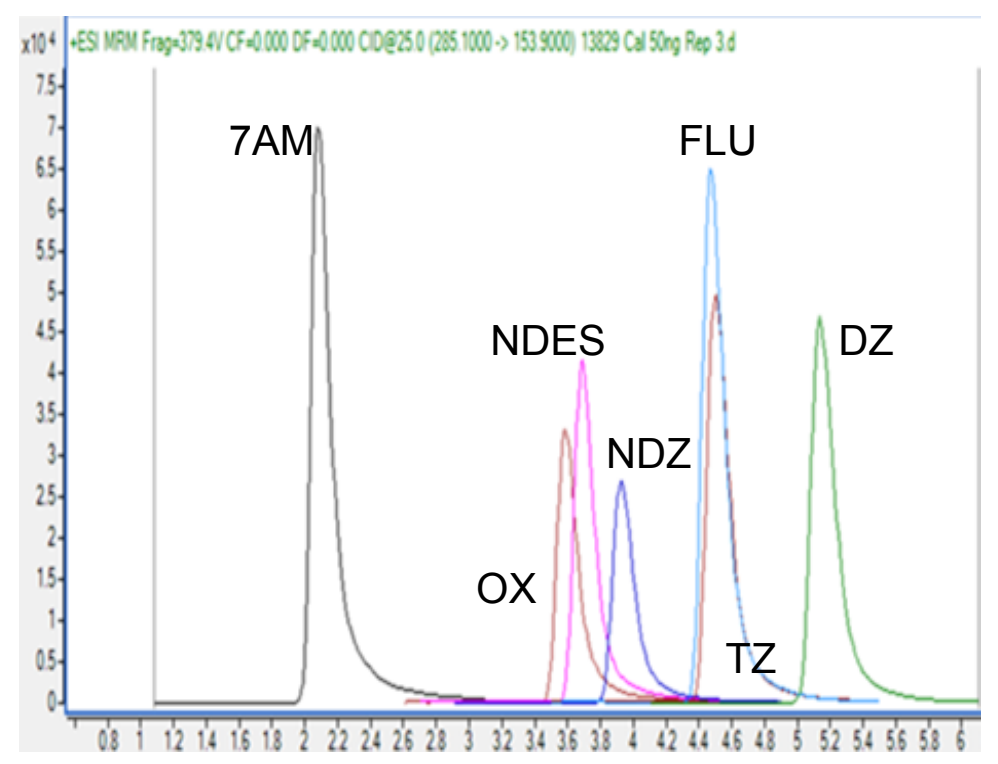

\subsubsection{Results and Discussion}

\subsubsection{Calibration Model}

Calibrators and quality control samples (QC) were prepared from the appropriate working solutions. The final calibration model consisted of a 10-point calibration at 0.05 , $0.1,0.5,1,5,10,50,100,250$, and $500 \mathrm{ng} / \mathrm{mL}$ for the analytes of interest and $50 \mathrm{ng} / \mathrm{mL}$ for the deuterated standards extracted from RCM. QCs were established at 1 (low) and $100 \mathrm{ng} / \mathrm{mL}$ (high). The calibration range, correlation coefficient, LOD and LOQ for each analyte can be seen in Table 18. The calibration model was one of the most important features of method validation because subsequent analyses and quantitation were based upon this model. 
Table 18 - Calibration Range, Correlation, LOD, and LOQ for Diazepam, Flunitrazepam, and Metabolites in RCM

\begin{tabular}{|ccccc|}
\hline Analyte & $\begin{array}{c}\text { Calibration Range } \\
(\mathbf{n g} / \mathbf{m L})\end{array}$ & $\mathbf{R}^{\mathbf{2}}$ & $\begin{array}{c}\text { LOD } \\
(\mathbf{n g} / \mathbf{m L})\end{array}$ & $\begin{array}{c}\text { LOQ } \\
(\mathbf{n g} / \mathbf{m L})\end{array}$ \\
\hline DZ & $0.5-500$ & 0.996 & 0.1 & 0.5 \\
\hline NDZ & $0.5-500$ & 0.996 & 0.5 & 0.5 \\
\hline OX & $1.0-500$ & 0.995 & 1.0 & 1.0 \\
\hline TZ & $0.5-500$ & 0.996 & 0.5 & 0.5 \\
\hline FLU & $0.5-500$ & 0.995 & 0.5 & 0.5 \\
\hline 7AM & $0.1-500$ & 0.996 & 0.1 & 0.1 \\
\hline NDES & $0.5-500$ & 0.995 & 0.5 & 0.5 \\
\hline
\end{tabular}

\subsubsection{Carryover Analysis}

Carryover analysis is a component of method validation advocated by SWGTOX.

Because some $L O Q$ values were as low as $0.1 \mathrm{ng} / \mathrm{mL}$, carryover effects from high concentration samples posed a real threat to the accuracy of subsequent sample quantitation. Carryover analysis was conducted at three concentrations including the highest calibration point of $500 \mathrm{ng} / \mathrm{mL}$ and two points at higher concentration: 700 $\mathrm{ng} / \mathrm{mL}$ and $900 \mathrm{ng} / \mathrm{mL}$. An extracted blank was injected after each sample to analyze carryover effects.

Analysis of previously established identification criteria as well as agreement between experimental and theoretical concentrations indicated that the presented method was valid up to at least $900 \mathrm{ng} / \mathrm{mL}$ using the linear calibration model. However, analysis of the blank injections proved that carryover was present for all target analytes at higher concentrations. To counteract the effects of carryover, an extracted blank sample was run after every sample injection. 


\subsubsection{Interference Studies}

Interference studies were a component of method specificity as defined in section 1.5.3. Ten samples of RCM matrix were pooled for validation interference studies. Blank matrix samples were extracted and analyzed to identify matrix effects that could potentially affect analysis of the analytes of interest. While there was some evidence of matrix effect based upon qualitative analysis, the established identification criteria of RT, peak shape, and ion ratios were not satisfied in blank specimens.

Blank matrix was also spiked at $50 \mathrm{ng} / \mathrm{mL}$ for each deuterated standard to address possible interferences arising from similar fragmentation patterns of target analyte and corresponding deuterated standard as well as potential internal standard impurities. The blank matrix samples containing deuterated standards likewise exhibited negligible interference based on the established identification criteria.

\subsubsection{Ionization Suppression/Enhancement and Recovery}

Ionization suppression and enhancement studies were combined with ME, RE, and PE studies. These were defined in Section 1.5.3.2 as components of method specificity. Samples were prepared in duplicate and analyzed in triplicate for Sets A, B, and $C$ at each concentration level. Equations 1-3 as defined in Chapter 1 were used to calculate $\% \mathrm{ME}, \% \mathrm{RE}$, and $\% \mathrm{PE}$ for each analyte. Results are summarized in Table 19. 
Table 19 - Matrix Effects, Recovery, and Process Efficiency for Diazepam, Flunitrazepam, and Metabolites in RCM

\begin{tabular}{|cccccccc|}
\hline Analyte & DZ & ND & OX & TZ & FLU & 7AM & NDES \\
\hline \multicolumn{7}{c|}{ High (100 $\mathbf{~ g / m L ) ~}$} \\
\hline ME (\%) & 99.1 & 97.1 & 97.2 & 97.6 & 96.9 & 98.8 & 97.9 \\
\hline RE (\%) & 95.3 & 97.0 & 96.4 & 94.7 & 95.4 & 97.8 & 95.5 \\
\hline PE (\%) & 94.5 & 94.2 & 93.7 & 92.4 & 92.5 & 96.6 & 93.5 \\
\hline \multicolumn{7}{c}{ Low (1 ng/mL) } \\
\hline ME (\%) & 92.2 & 118.9 & 87.6 & 93.8 & 91.1 & 94.8 & 99.3 \\
\hline RE (\%) & 119.5 & 117.8 & 117.9 & 118.2 & 114.6 & 116.5 & 117.6 \\
\hline PE (\%) & 110.2 & 140.1 & 103.4 & 110.9 & 104.4 & 110.4 & 116.8 \\
\hline
\end{tabular}

Matrix effects ranged from $97-99 \%$ at high concentrations but demonstrated greater variation at lower concentrations from $91-119 \%$. At low concentration, all of the target analytes exhibited ionization suppression from interfering compounds in the matrix except for NDZ, which exhibited ionization enhancement. The suppression and enhancement effects were likely due to compounds within the RCM matrix that coeluted with the target analytes thereby affecting the ionization efficiency.

Recovery ranged from $95-98 \%$ at high concentration. At low concentration, all of the analytes exhibited recoveries over $100 \%$, ranging from $115-119 \%$. This indicated that samples spiked with analytes and deuterated standards prior to extraction (Set C) exhibited higher experimental concentrations than samples spiked after the extraction process (Set B). This was likely due to matrix components that were not removed during SPE. This explanation was supported by the ionization enhancement effects seen in \% ME at low concentrations.

Analytical recovery was representative of the effectiveness of the sample preparation technique. Strata-X-Drug $\mathrm{N}$ cartridges were chosen for their selectivity 
towards neutral drugs of abuse such as benzodiazepines. The SPE method included an aqueous wash step with acetonitrile/water $(20 / 80 \mathrm{v} / \mathrm{v})$ which helped to remove much of the salt content of the media. Samples were eluted with EA/IPA $(85 / 15 \mathrm{v} / \mathrm{v})$, which enhanced the separation of remaining polar compounds in the matrix from the analytes

of interest. Since the SPE method focused on removal of polar matrix components and salts, the recovery enhancement was likely due to nonpolar media components coeluting with the analytes of interest.

PE compared the spiked samples that went through the full extraction method (C) to the samples prepared in neat buffer (A). These values and ranged from $92-97 \%$ at high concentrations and $103-140 \%$ at lower concentrations. PE can also be considered as the combined effects of ME $\times$ RE, which accounted for the greater range of variation, especially for NDZ which was more sensitive to matrix effects.

\subsubsection{Bias and Precision}

Bias and precision studies were studied at four representative concentrations 0.500 (low), 1 (medium/low), 50 (medium), and 500 (high) ng/mL for DZ, NDZ, TZ, FLU, 7AM, and NDES, and three concentrations 1 (low), 50 (intermediate), and $500 \mathrm{ng} / \mathrm{mL}$ (high) for OX. Analysis was adjusted for OX because the LOD and LOQ values were higher than the other analytes at $1 \mathrm{ng} / \mathrm{mL}$. Five samples were prepared and extracted for each concentration and analyzed in triplicate.

The bias of the method was defined in Section 1.5.5 and calculated using Equation 5. Generally, the higher concentration sample sets had the narrowest range of values, which became broader as the concentration decreased toward analyte LOQ. Even though the acceptable bias range of $\pm 20 \%$ is the same for all concentration levels, 
small changes in sample extraction and preparation as well as instrumental response had a greater effect on lower concentrations than higher concentrations, causing the range of values to broaden.

Precision and repeatability of the method were determined using the same data set as bias analysis. Precision was defined in Section 1.5.6 and calculated as withinrun precision via Equation 6. Repeatability of the method was defined in Section 1.5.7 and calculated as between-run precision using Equation 7. The acceptable range for both bias and precision was $\pm 20 \%$ and values were recorded in Table 20 . Both sets of precision values followed the same trend as that seen with bias with narrowest range of values seen at higher concentrations. 
Table 20 - Bias, Within-Run and Between-Run \%CV for Diazepam, Flunitrazepam, and Respective Metabolites in RCM

\begin{tabular}{|c|c|c|c|c|c|c|c|}
\hline \multicolumn{8}{|c|}{ Bias and Precision } \\
\hline Analyte & DZ & ND & ox & TZ & FLU & $7 \mathrm{AM}$ & NDES \\
\hline \multicolumn{8}{|c|}{ High (500 ng/mL) } \\
\hline Bias & -2.1 & -3.6 & -3.0 & -2.0 & -3.4 & -2.5 & -1.7 \\
\hline Within-Run & 1.8 & 2.3 & 1.8 & 2.0 & 2.6 & 1.5 & 2.3 \\
\hline Between-Run & 2.8 & 2.8 & 2.7 & 2.7 & 2.9 & 2.9 & 3.8 \\
\hline \multicolumn{8}{|c|}{ Medium (50 ng/mL) } \\
\hline Bias & -3.6 & -5.5 & -9.9 & -8.5 & -10.5 & -5.5 & -8.1 \\
\hline Within-Run & 1.7 & 2.9 & 1.8 & 2.4 & 1.9 & 1.9 & 2.0 \\
\hline Between-Run & 2.8 & 3.6 & 2.1 & 3.4 & 2.7 & 2.5 & 3.4 \\
\hline \multicolumn{8}{|c|}{ Medium/Low (1 ng/mL) } \\
\hline Bias & -8.9 & 1.3 & -12.1 & -17.6 & -14.4 & -8.0 & -10.7 \\
\hline Within-Run & 4.4 & 4.4 & 11.3 & 5.0 & 5.2 & 2.1 & 5.5 \\
\hline Between-Run & 5.4 & 5.9 & 11.7 & 5.9 & 5.9 & 4.0 & 5.0 \\
\hline \multicolumn{8}{|c|}{ Low $(0.500 \mathrm{ng} / \mathrm{mL})$} \\
\hline Bias & -5.7 & -6.8 & NA & -9.7 & -13.1 & -14.9 & -4.5 \\
\hline Within-Run & 3.6 & 4.7 & N/A & 10.5 & 10.2 & 2.1 & 12.2 \\
\hline Between-Run & 4.6 & 6.0 & N/A & 8.1 & 6.6 & 4.0 & 9.5 \\
\hline
\end{tabular}

\subsubsection{Dilution Integrity}

To determine dilution integrity (Section 1.5.11), bias and precision studies were repeated for the diluted samples. This was an important feature of method validation because biotransformation samples were spiked at $1000 \mathrm{ng} / \mathrm{mL}$ (Chapter 4). Common dilution ratios of $1: 2$ for an $800 \mathrm{ng} / \mathrm{mL}$ sample and $1: 5$ for a $1000 \mathrm{ng} / \mathrm{mL}$ sample were examined. Five samples per concentration level were prepared, extracted, and analyzed in triplicate. Both sets of dilutions maintained the require identification criteria. Bias ranged from -8.1 to $7.9 \%$ across both dilution sets while within-run precision 
ranged from $1.8-5.4 \%$ and between run precision from $2.1-3.9 \%$. Bias and precision data for the dilution samples was summarized in Table 21. Future dilutions could be prepared at either $1: 2$ or 1:5 and maintain sample integrity.

Table 21 - Dilution Integrity for 1:2 and 1:5 Sample Dilutions

\begin{tabular}{|crrrrrrr|}
\hline \multicolumn{7}{c|}{ Dilution Integrity } \\
\multicolumn{7}{c|}{$\mathbf{1 : 2}(\mathbf{8 0 0}: \mathbf{4 0 0} \mathbf{~ n g} / \mathbf{m L})$} \\
\hline Bias & 0.55 & 6.7 & 2.7 & -2.6 & -1.9 & -5.6 & -6.9 \\
\hline Within-Run & 1.8 & 3.6 & 2.7 & 3.9 & 4.3 & 3.2 & 4.0 \\
\hline Between-Run & 2.1 & 2.2 & 2.7 & 2.4 & 3.1 & 2.2 & 2.6 \\
\hline \multicolumn{7}{c}{$\mathbf{1 : 5 ( 1 0 0 0 : 2 0 0}$} & $\mathbf{n g} / \mathbf{m L})$ \\
\hline Bias & 1.4 & 7.9 & 1.5 & -3.3 & -1.1 & -3.4 & -8.1 \\
\hline Within-Run & 4.4 & 3.2 & 4.8 & 5.4 & 3.8 & 4.0 & 3.3 \\
\hline Between-Run & 3.1 & 3.6 & 3.9 & 3.4 & 2.6 & 3.0 & 3.3 \\
\hline
\end{tabular}

\subsubsection{LOD and LOQ}

The LOD and LOQ criteria are summarized in sections 1.5.8 and 1.5.9, respectively. Values ranged from 0.100 to $1 \mathrm{ng} / \mathrm{mL}$ for DZ, FLU, and metabolites. Results were included in Table 18. DZ and 7AM achieved the lowest LOD and LOQ values at $0.1 \mathrm{ng} / \mathrm{mL}$. However, while DZ achieved acceptable RT, peak shape, and ion ratios at $0.1 \mathrm{ng} / \mathrm{mL}$ which satisfied LOD requirements, the bias exceeded $\pm 20 \%$. LOQ requirements were fulfilled at $0.5 \mathrm{ng} / \mathrm{mL}$ for $\mathrm{DZ}$. The remaining analytes of interest achieved $L O Q$ values $\leq 0.5 \mathrm{ng} / \mathrm{mL}$ with the exception of $O X$.

OX samples were within the appropriate RT window and maintained good peak shape. However, the ion ratio analysis prevented detection and quantitation limits from being confirmed with certainty at lower concentrations. This was supported by data obtained for interference analysis in Section 3.3.4. The \% ME decreased from $97.2 \%$ at 
high concentration to $87.6 \%$ at low concentration, indicating that ionization suppression of OX was greater at low concentrations.

Additionally, the \% RE increased from $96.4 \%$ (high) to $117.9 \%$ (low). This supported the inconsistent ion ratios seen in analysis of LOD and LOQ determination. The accuracy and bias of OX samples at low concentrations were within the acceptable $\pm 20 \%$. However, the ion ratios at low concentrations increased. Ion ratios were calculated as a ratio of response between qualitative ion: quantitative ion. Increase in ion ratio indicated an increase in response of the $162.9 \mathrm{~m} / \mathrm{z}$ transition in comparison to that of the $241.1 \mathrm{~m} / \mathrm{z}$ transition. This could have been indicative of a matrix component with a similar set of ion transitions, which affected response of OX.

\subsubsection{Stability}

The FT and processed sample stability were both assessed as part of the method validation. FT stability was conducted at two concentrations $500 \mathrm{ng} / \mathrm{m}$ (high) and $1 \mathrm{ng} / \mathrm{mL}$ (low). The FT cycle analysis for $500 \mathrm{ng} / \mathrm{mL}$ was expressed in Figure 14 . 
Figure 14 - Freeze-Thaw Stability for Diazepam, Flunitrazepam, and Respective Metabolites in RCM at $500 \mathrm{ng} / \mathrm{mL}$

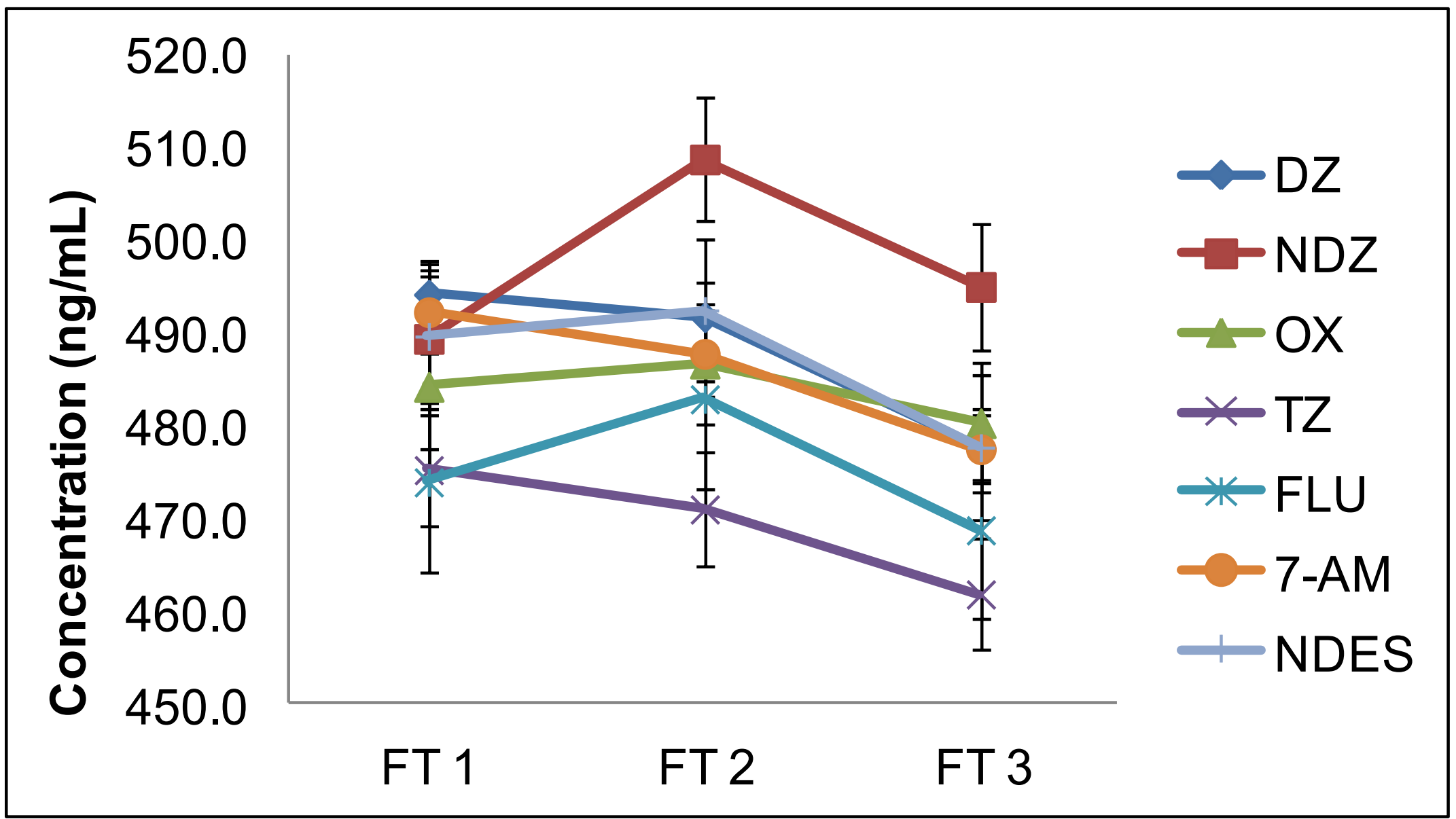


At high concentration the acceptable range of $\pm 20 \%$ was between $400-600$ $\mathrm{ng} / \mathrm{mL}$. NDZ, OX, FLU, NDES exhibited a slight increase in response between FT1 and FT2 while the other compounds showed a decrease. Since an extracted matrix blank was injected between each sample, these effects were not due to carryover. The results were likely due to fluctuations in instrumental response. All analyte concentrations decreased from FT2 to FT3, which was expected. However none of the compounds fell below $400 \mathrm{ng} / \mathrm{mL}$, which indicated that samples would retain stability through 3 FT cycles at high concentration.

The freeze-thaw cycle for $1 \mathrm{ng} / \mathrm{mL}$ samples was depicted in Figure 15. At low concentration the acceptable range was between 0.8 and $1.2 \mathrm{ng} / \mathrm{mL}$. NDZ and NDES again displayed an increase in response between FT1 and FT2 while the other analytes decreased in concentration. All analyte concentrations decreased between FT2 and FT3. Unlike what was seen at high concentration, responses for OX, TZ, and FLU fell below $0.8 \mathrm{ng} / \mathrm{mL}$. For this method, it was therefore concluded that samples may undergo up to two FT cycles and still maintain stability criteria, but cannot be analyzed after three FT cycles with certainty. 
Figure 15 - Freeze-Thaw Stability for Diazepam, Flunitrazepam, and Respective Metabolites in RCM at $1 \mathrm{ng} / \mathrm{mL}$

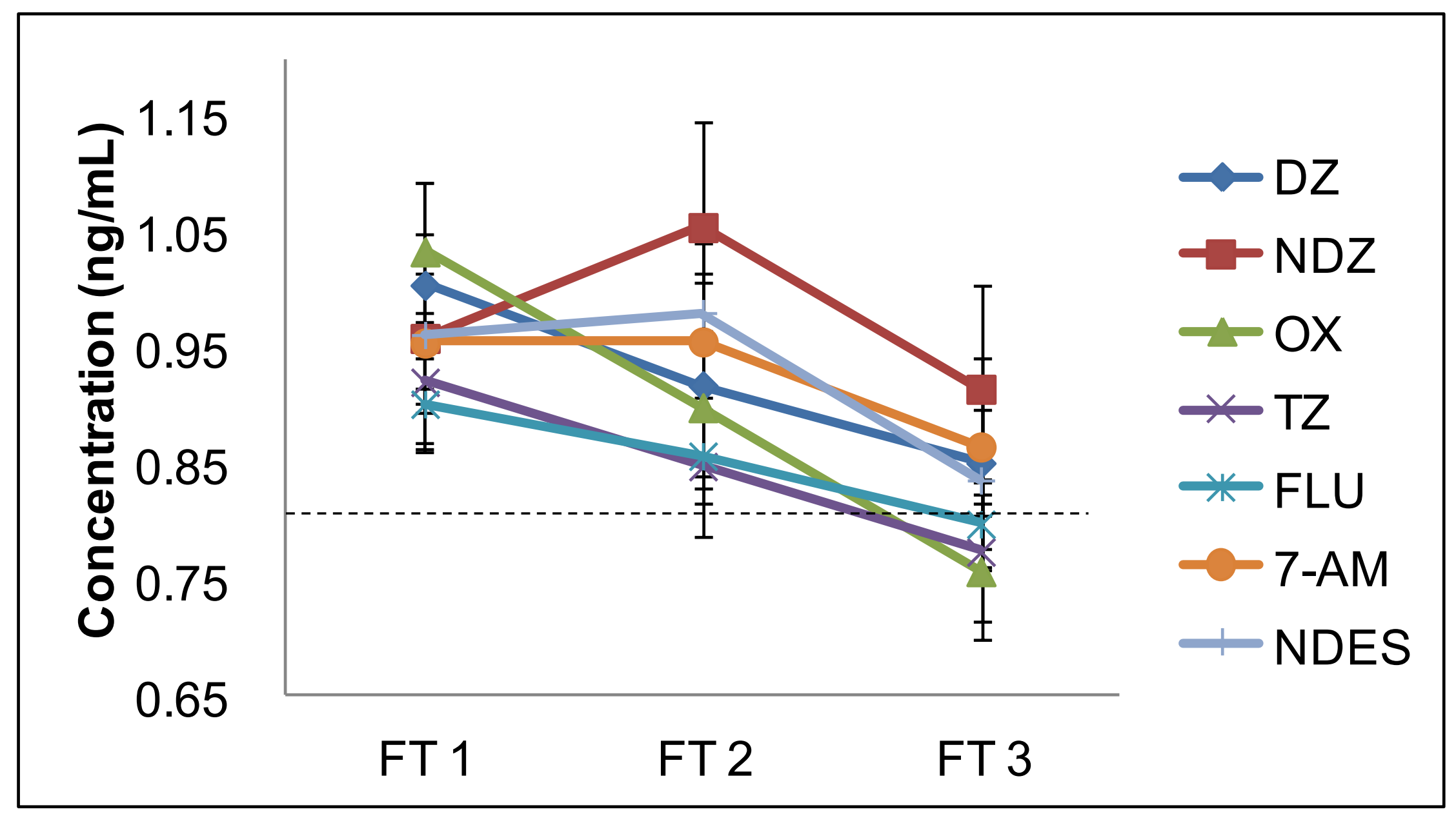


The stability of processed samples was studied to address possible effects of large sample batches or instrumental malfunctions that would prevent samples from being analyzed immediately. Stability studies were conducted at two concentrations $500 \mathrm{ng} / \mathrm{mL}$ (high) and $0.500 \mathrm{ng} / \mathrm{mL}$ (low). Two samples were prepared in RCM at each concentration, extracted, reconstituted, and analyzed in triplicate over 48 hours. Eleven samples were run at time $0,3,6,9,12,15,18,24,30,36$, and 48 hours. For high concentration, analyte response ranged from FLU at $477 \mathrm{ng} / \mathrm{mL}$ to $\mathrm{OX}$ at $563 \mathrm{ng} / \mathrm{mL}$. The processed samples remained stable for at least 48 hours for all analytes of interest. Results can be seen in Figure 16.

For low concentration samples at time 48 hours, responses ranged from 7AM at $0.462 \mathrm{ng} / \mathrm{mL}$ and DZ at $0.534 \mathrm{ng} / \mathrm{mL}$. Results can be seen in Figure 17. Based on the processed sample stability studies, all analytes of interest maintained stability for at least 48 hours. 
Figure 16 - Processed Sample Stability for Diazepam, Flunitrazepam, and Respective Metabolites in RCM at $500 \mathrm{ng} / \mathrm{mL}$

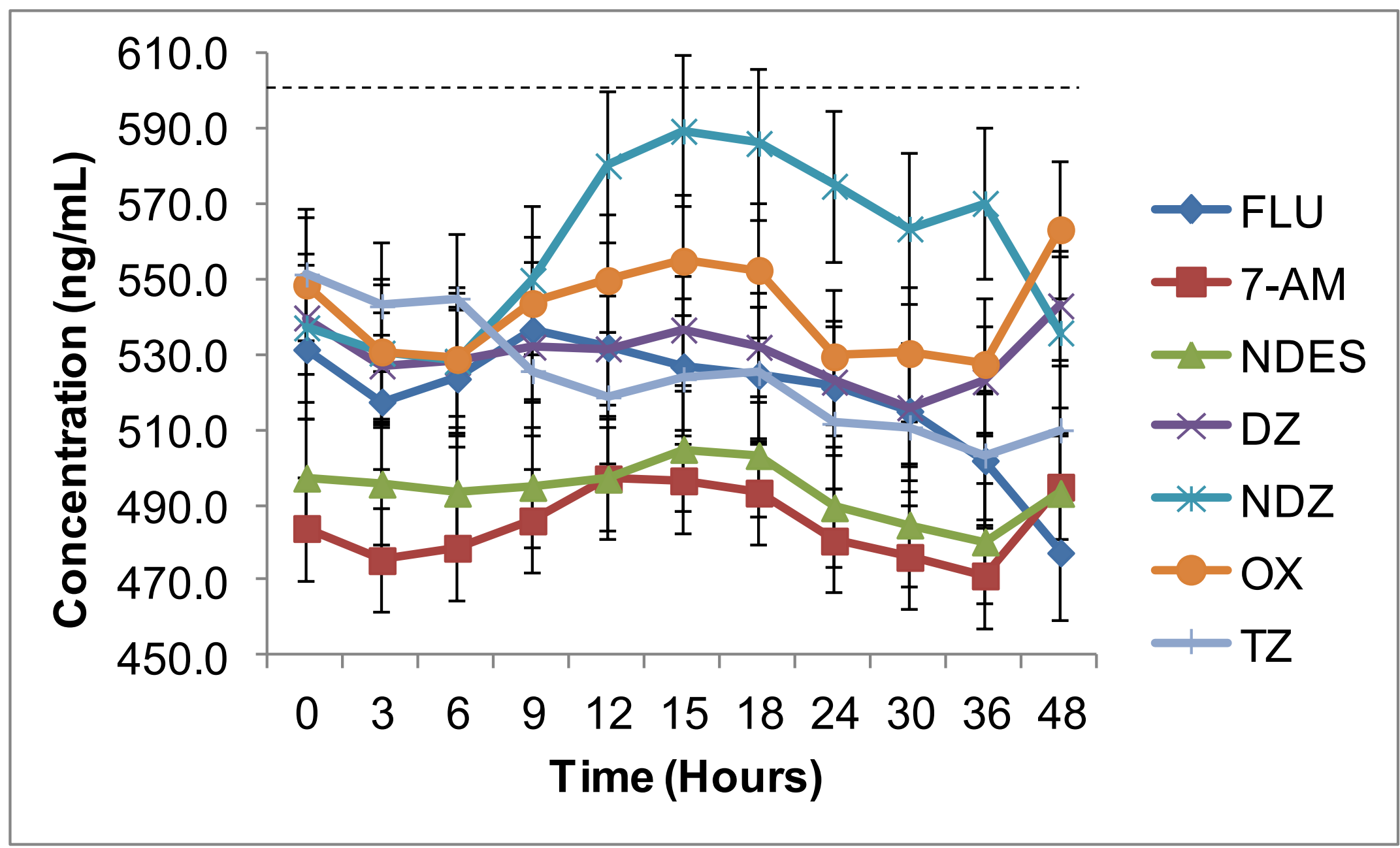


Figure 17 - Processed Sample Stability for Diazepam, Flunitrazepam, and Respective Metabolites in RCM at $0.5 \mathrm{ng} / \mathrm{mL}$

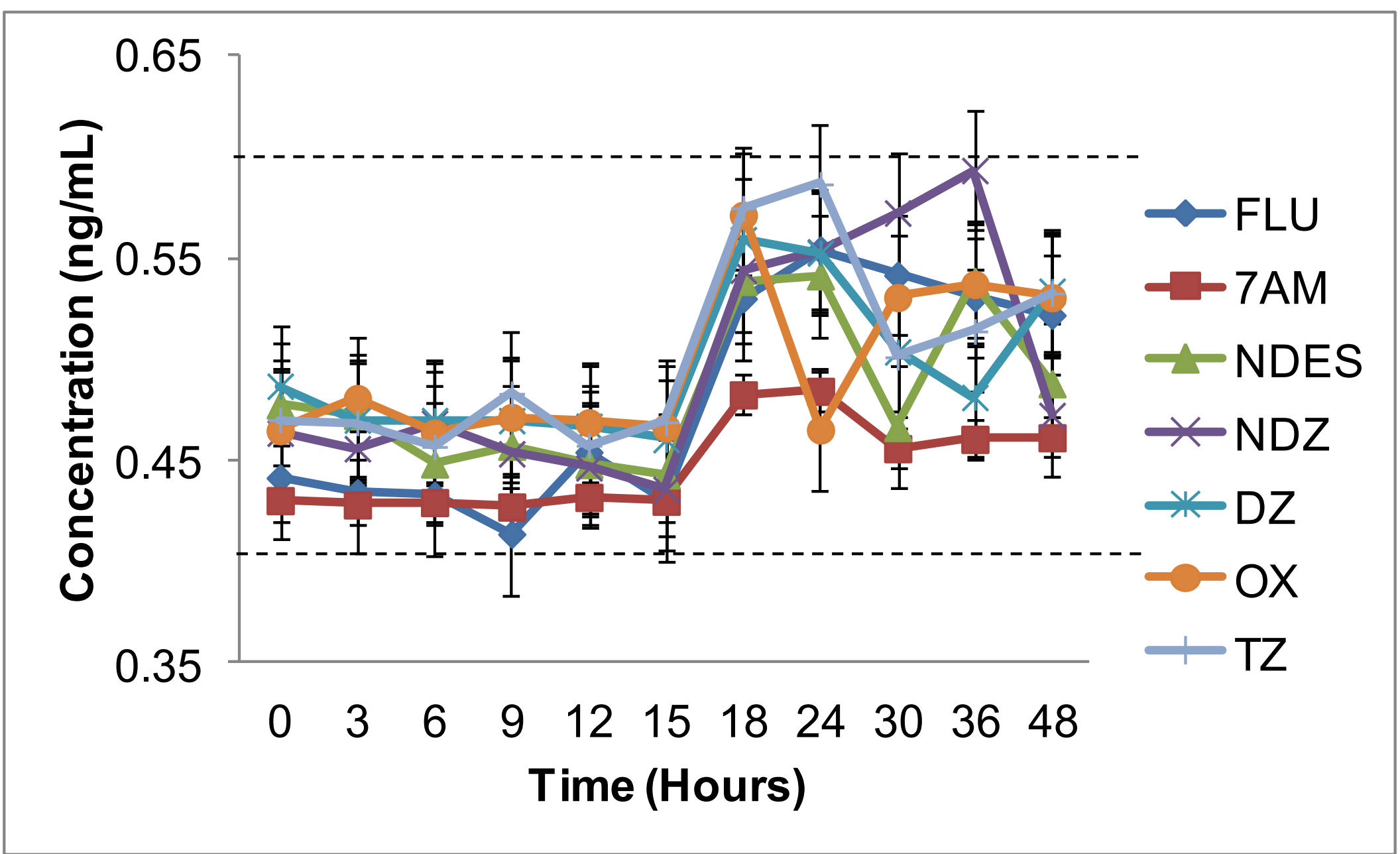




\subsubsection{Robustness}

Robustness of the method was defined in Section 1.5.13 and determined by slight changes in the buffer composition and column temperature. Samples were run at $0.5,5,50,250$, and $500 \mathrm{ng} / \mathrm{mL}$ for each variable. Buffer $\mathrm{A}$ for this benzodiazepine method consists of water with $0.1 \%$ formic acid. The effect of changes in buffer composition was assessed by running a series of samples with buffers containing $0.05 \%, 0.1 \%$, and $0.15 \%$ formic acid. Samples with the $0.05 \%$ and $0.1 \%$ formic acid buffer maintained identification criteria and experimental concentrations were within $\pm 20 \%$ for all concentration levels.

The $0.15 \%$ formic acid samples maintained identification and accuracy criteria for $5,50,250$, and $500 \mathrm{ng} / \mathrm{mL}$ but ranged from $108-141 \%$ for the $0.5 \mathrm{ng} / \mathrm{mL}$ sample. The same samples were used for the buffer composition studies, so this effect was not due to differences in sample preparation or extraction. RT, peak shapes, and ion ratios all experienced more fluctuation with this buffer composition for all analytes. Peak shapes at the lowest concentration of $0.5 \mathrm{ng} / \mathrm{mL}$ were no longer Gaussian which affected the accuracy of quantitation.

Column temperature for the validated method was held at $37^{\circ} \mathrm{C}$. The effects of column temperature were analyzed at $30^{\circ} \mathrm{C}, 37^{\circ} \mathrm{C}$, and $40^{\circ} \mathrm{C}$. Identification and accuracy criteria were met for DZ, OX, TZ, FLU, 7AM, and NDES for all concentration levels and temperatures. The $0.5 \mathrm{ng} / \mathrm{mL}$ sample for $\mathrm{OX}$ at $40^{\circ} \mathrm{C}$ robustness study exceeded the $\pm 20 \%$ theoretical concentration allotment at $134 \%$. However, the LOD and LOQ for OX were $1 \mathrm{ng} / \mathrm{mL}$, so this analyte could not have been detected or 
quantified at $0.5 \mathrm{ng} / \mathrm{mL}$ with certainty using this method regardless of column temperature.

In addition to buffer composition and column temperature, an alternate analyst prepared samples at the five concentration levels for analysis. Identification criteria were met at all concentration levels.

\subsubsection{Conclusions}

Validation of this method was essential for further studies on the biotransformation of drugs of abuse by bacteria grown in RCM. This LC-MS/MS method was used to analyze and quantify the bacterial biotransformation phenomena presented in Chapter 4. It will also be used to compare the bacterial products to those of normal human metabolism. 


\section{Chapter 4: Detection and Quantitation of Diazepam and Flunitrazepam Metabolites Produced by Anaerobic Bacterial Species in Reinforced Clostridial Medium using Liquid Chromatography-Tandem Mass Spectrometry}

\subsubsection{Introduction}

The ability of bacterial species to transform a variety of complex compounds has been well documented. Some of these species are native to the human GI tract and may have the potential to cause biotransformations that affect the ratio of compound-tometabolite concentrations within the human body. Investigation of these possibilities required optimization of bacterial growth conditions, a method of analyte extraction from the nutrient medium RCM, and an LC-MS/MS method for analysis and quantitation of experimental results. Bacterial growth conditions and methods were optimized in Chapter 2 of this document, and the SPE and LC-MS/MS methods were developed and validated in Chapter 3.

Chapter 4 is essentially the culmination and application of the previous work.

Experiments focused on the effects of E. coli, B. fragilis, and C. perfringens on DZ, FLU, and their metabolites in RCM under anaerobic conditions. DZ and FLU are benzodiazepine compounds of forensic importance and interest. They were selected for biotransformation studies because the human metabolites have been previously identified and reference standards were commercially available. 


\subsubsection{Diazepam Metabolism}

Human metabolites of DZ include nordiazepam (NDZ), oxazepam (OX), and temazepam (TZ) and can be seen in Figure $17 .{ }^{46,48,50-52,56,60-62}$

Figure 18 - Human Metabolites of Diazepam

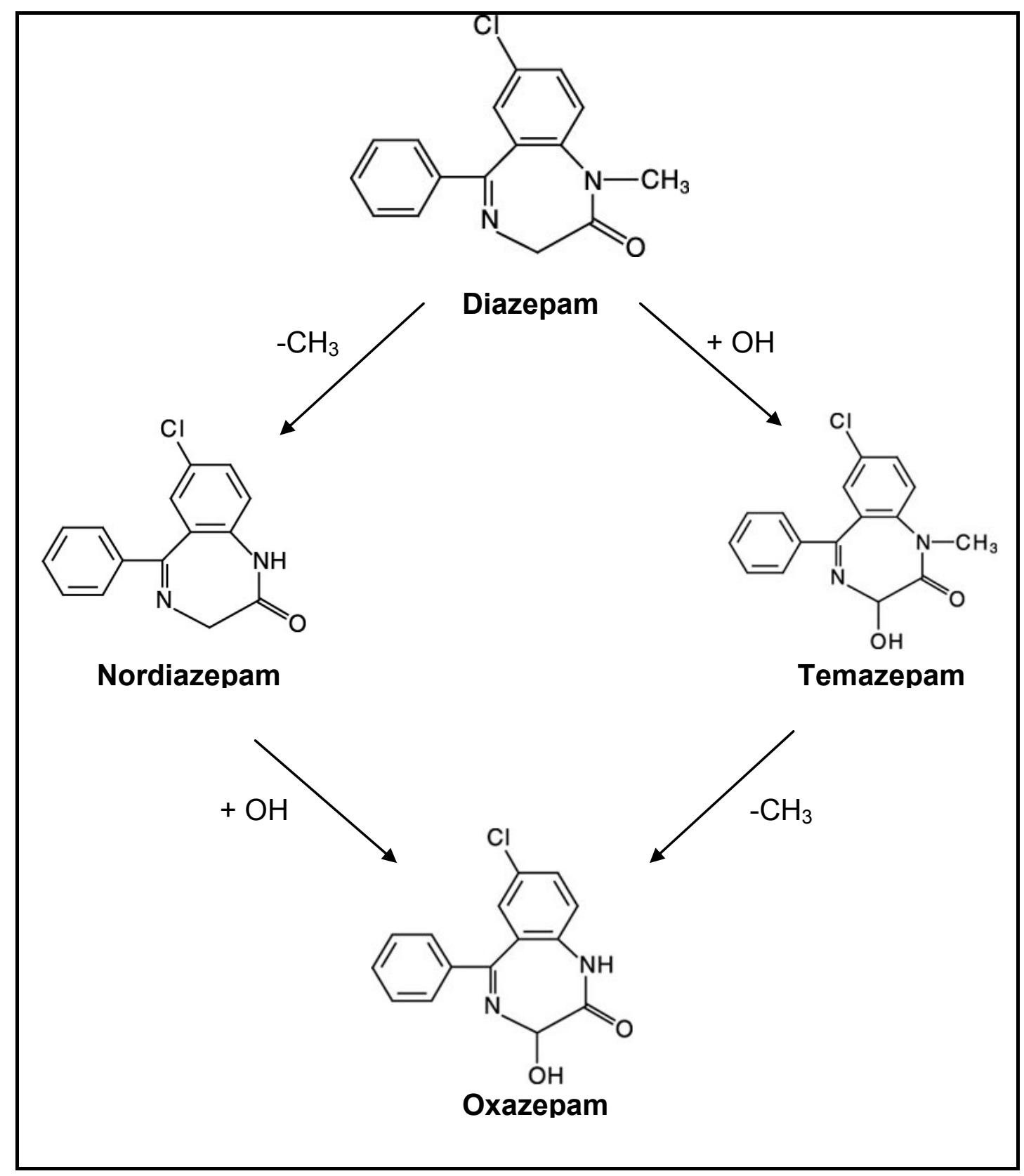




\subsubsection{Flunitrazepam Metabolism}

Human metabolites of FLU include 7-aminoflunitrazepam (7AM) and $\mathrm{N}$ desmethylflunitrazepam (NDES) and can be seen in Figure $18 .{ }^{47,48,51,53,57-59}$

The 3-hydroxyflunitrazepam metabolite was not included in analysis because it was not available at the time of method development and validation.

Figure 19 - Human Metabolites of Flunitrazepam

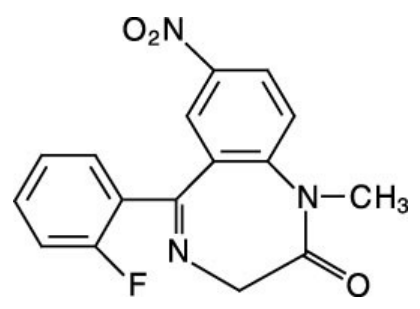

Flunitrazepam

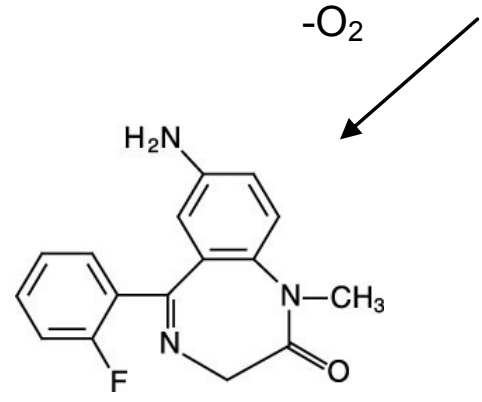

7-Aminoflunitrazepam

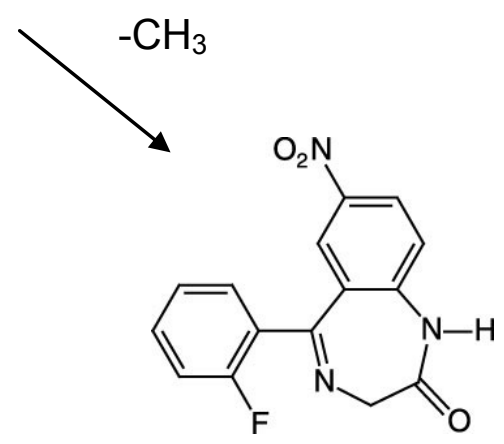

$N$-Desmethylflunitrazepam

\subsubsection{Materials}

Escherichia coli (33985), Bacteroides fragilis (29771), and Clostridium perfringens (19574) were purchased from the American Type Culture Collection® (Manassas, VA). BD Difco ${ }^{\mathrm{TM}}$ Reinforced Clostridial Medium and sterile $15 \mathrm{~mL}$ 
polypropylene BD Falcon ${ }^{\mathrm{TM}}$ tubes were obtained from BD Diagnostic Systems (Sparks, MD). The Vinyl Anaerobic Chamber (Type A) with automatic airlock and incubator produced by Coy Laboratory Products Inc was used for anaerobic incubation (Grass Lake, MI). Reference standards were purchased from Cerilliant ${ }^{\circledR}$ (Round Rock, TX) as $1.0 \mathrm{mg} / \mathrm{mL}$ for DZ and FLU. All other materials used in extraction and LC-MS/MS analysis were detailed in Section 3.2.0.

\subsubsection{Methods}

\subsubsection{Conditions}

E.coli, B. fragilis, and C. perfringens required specific environmental growth conditions. These were optimized and discussed in Section 2.6.0.

\subsubsection{Media}

The RCM was prepared according to manufacturer instructions by dissolving 38 grams of dehydrated RCM in purified water. The medium was autoclaved at $121^{\circ} \mathrm{C}$ for 15 minutes. RCM was transferred to the anaerobic chamber and stored with the cap loosened for 48 hours to promote evacuation of dissolved oxygen. This was particularly important for growth of C. perfringens. After evacuation, RCM was capped and stored at room temperature until further use.

\subsubsection{Bacterial Sample Preparation}

One of the frozen stock cultures described in Section 2.7.0 was removed from storage and placed in the anaerobic chamber. The contents were transferred to a 
sterile $15 \mathrm{~mL}$ tube containing $10 \mathrm{~mL}$ of $\mathrm{RCM}$ and incubated with the lid loosened at $37^{\circ} \mathrm{C}$ for 48 hours. This process was repeated for all three bacterial species.

Blanks and biotransformation samples were all prepared in $10 \mathrm{~mL}$ of RCM. They were dosed with analyte and/or bacterial specimen as outlined in Table 22.

Immediately after the samples were prepared, they were vortexed for 30 seconds, inverted 10 times, and vortexed for an additional 30 seconds to ensure thorough mixing. A $200 \mu \mathrm{L}$ pre-incubation aliquot of each sample was transferred to an Eppendorf tube and transported out of the anaerobic chamber for analysis. Samples were incubated with the lid loosened at $37^{\circ} \mathrm{C}$ for 48 hours.

Table 22 - Sample Preparation

\begin{tabular}{|c|ccccc|}
\hline Sample & $\begin{array}{c}\mathbf{D Z} \\
(\mathrm{ng} / \mathrm{mL})\end{array}$ & $\begin{array}{c}\text { FLU } \\
(\mathrm{ng} / \mathrm{mL})\end{array}$ & $\begin{array}{c}\text { E. coli } \\
(\mu \mathrm{L})\end{array}$ & $\begin{array}{c}\text { B. fragilis } \\
(\mu \mathrm{L})\end{array}$ & $\begin{array}{c}\text { C. perfringens } \\
(\mu \mathrm{L})\end{array}$ \\
\hline DZ Blank & 1000 & - & - & - & - \\
\hline FLU Blank & - & 1000 & - & - & - \\
\hline E. coli Blank & - & - & 250 & - & - \\
\hline B. fragilis Blank & - & - & - & 250 & - \\
\hline C. perfringens Blank & - & - & - & - & 250 \\
\hline Mixed Culture Blank & - & - & 100 & 100 & 100 \\
\hline DZ + E.coli & 1000 & - & 250 & - & - \\
\hline DZ + B. fragilis & 1000 & - & - & 250 & - \\
\hline DZ + C. perfringens & 1000 & - & - & - & 250 \\
\hline DZ + Mixed Culture & 1000 & - & 100 & 100 & 100 \\
\hline FLU + E.coli & - & 1000 & 250 & - & - \\
\hline FLU + B. fragilis & - & 1000 & - & 250 & - \\
\hline FLU + C. perfringens & - & 1000 & - & - & 250 \\
\hline FLU + Mixed Culture & - & 1000 & 100 & 100 & 100 \\
\hline
\end{tabular}

The $200 \mu \mathrm{L}$ pre-incubation aliquot (concentration $1000 \mathrm{ng} / \mathrm{mL}$ ) was combined with $10 \mu \mathrm{L}$ of internal standard working solution $(5000 \mathrm{ng} / \mathrm{mL})$ and $790 \mu \mathrm{L}$ water/acetonitrile (60/40 v/v) buffer for a $1: 5$ dilution (Section 3.3.6). Pre-incubation samples were immediately extracted and then analyzed via LC-MS/MS. 
Post-incubation, samples were removed from the chamber after 48 hours, vortexed for 30 seconds, inverted 10 times, and vortexed for an additional 30 seconds to ensure uniform composition throughout. The OD was measured for comparison to the values obtained during the plate count method. Another $200 \mu \mathrm{L}$ aliquot of each sample was extracted and analyzed as the post-incubation counterpart. Instrumental parameters, MRM transitions, SPE, and the LC-MS/MS method of analysis are all described in Chapter 3.

\subsubsection{Results and Discussion}

Two sets of DZ and FLU biotransformation experiments were conducted while the LC-MS/MS method was being developed and validated. Samples were prepared following the same guidelines as those described in Table 22 and stored in the freezer until they could be extracted and analyzed. These sets of experiments were analyzed but both of them were rendered useless.

In the first set, nearly all of the media samples inoculated for biotransformation studies failed to support bacterial growth. E. coli was the only specimen to produce any sort of turbidity, as evidenced by the resultant OD values. However, even these samples were barely distinguishable from the RCM blanks. Purging RCM in the anaerobic chamber for 48 hours after sterilization eliminated this problem, and all of the bacterial species demonstrated prominent growth.

The second set of samples was analyzed via LC-MS/MS. However, there was

no way to determine the original concentrations of the samples prior to incubation. Any evidence of biotransformation could not be definitively attributed to the bacterial 
species. Decrease in theoretical concentration could have been a result of incomplete dosing. It could also have been due to unassisted degradation of the analyte under experimental conditions. This dilemma was solved by implementing analysis of preincubation as well as post-incubation aliquots of the samples.

\subsubsection{Bacterial Blanks}

A series of bacterial blanks were prepared with aliquots of $E$. coli, B. fragilis, $C$. perfringens, or a mixture of the three without analytes of interest. None of the bacterial specimens produced interferences with the target analytes that satisfied identification and confirmation requirements of peak shape, retention time, ion ratio, and that were above the analyte LOQ.

\subsubsection{Diazepam Blanks}

A series of analyte blanks was prepared in RCM and incubated alongside samples inoculated with bacteria. Two blanks per analyte were spiked with either DZ or FLU and incubated at $37^{\circ} \mathrm{C}$ for 48 hours. Samples were evaluated via OD measurement to ensure that the media remained clear and turbidity-free over the course of the experiments. Pre- and post-incubation aliquots were extracted and analyzed via LC-MS/MS. Analytes and metabolites were evaluated for acceptable peak shape, retention time, ion ratios, and $\mathrm{S} / \mathrm{N} \geq 3$ for detection, and with bias and precision within $\pm 20 \%$ for quantitation.

Analysis of DZ Drug Blank 1 (Table 23) demonstrated an average change in concentration of $-11.1 \pm 3.2 \mathrm{ng} / \mathrm{mL}$ and DZ Drug Blank 2 (Table 24) an average difference of $-21.4 \pm 3.6 \mathrm{ng} / \mathrm{mL}$. However, NDZ, OX, nor TZ registered an increase from 
pre- to post-incubation concentrations to account for the DZ conversion. Standard deviations were determined at the $95 \%$ confidence interval.

The RCM for both DZ blank samples maintained its clarity and did not exhibit any characteristics that would indicate bacterial presence. The discrepancy in mass balance between pre- and post-incubation samples suggested that DZ must undergo a partial, unassisted degradation to a compound other than those found in normal human metabolism. This was likely due to molecule instability when incubated at $37^{\circ} \mathrm{C}$ for 48 hours.

Table 23 - Pre- and Post-Incubation Analysis: Diazepam Drug Blank 1

\begin{tabular}{|ccccc|}
\hline & \multicolumn{4}{c|}{ Concentration $(\mathbf{n g} / \mathbf{m L})$} \\
\hline Sample & DZ & NDZ & OX & TZ \\
\hline Pre DZ Blank 1 & $165.3 \pm 0.5$ & $0.1 \pm 0.0$ & $0.0 \pm 0.0$ & $0.0 \pm 0.0$ \\
\hline Post DZ Blank 1 & $154.2 \pm 3.1$ & $0.0 \pm 0.0$ & $0.0 \pm 0.0$ & $0.0 \pm 0.0$ \\
\hline$\Delta$ Concentration Average & $-11.1 \pm 3.2$ & $0.0 \pm 0.0$ & $0.0 \pm 0.0$ & $0.0 \pm 0.0$ \\
\hline
\end{tabular}

Table 24 - Pre- and Post-Incubation Analysis: Diazepam Drug Blank 2

\begin{tabular}{|ccccc|}
\hline & \multicolumn{4}{c|}{ Concentration $(\mathbf{n g} / \mathbf{m L})$} \\
\hline Sample & $\mathbf{D Z}$ & NDZ & OX & TZ \\
\hline Pre DZ Blank 2 & $187.9 \pm 2.6$ & $0.1 \pm 0.0$ & $0.0 \pm 0.0$ & $0.0 \pm 0.0$ \\
\hline Post DZ Blank 2 & $166.4 \pm 1.2$ & $0.0 \pm 0.0$ & $0.0 \pm 0.0$ & $0.0 \pm 0.0$ \\
\hline$\Delta$ Concentration Average & $-21.4 \pm 3.6$ & $0.0 \pm 0.0$ & $0.0 \pm 0.0$ & $0.0 \pm 0.0$ \\
\hline
\end{tabular}

A literature search of previously published research identified several possibilities for alternative metabolites including 4'-OH DZ (metabolite of DZ) and 4'-OH norezepam, a metabolite of NDZ. ${ }^{63}$ The metabolic scheme was depicted in Figure 20. 
Figure 20 - Alternative Metabolites of Diazepam and Nordiazepam

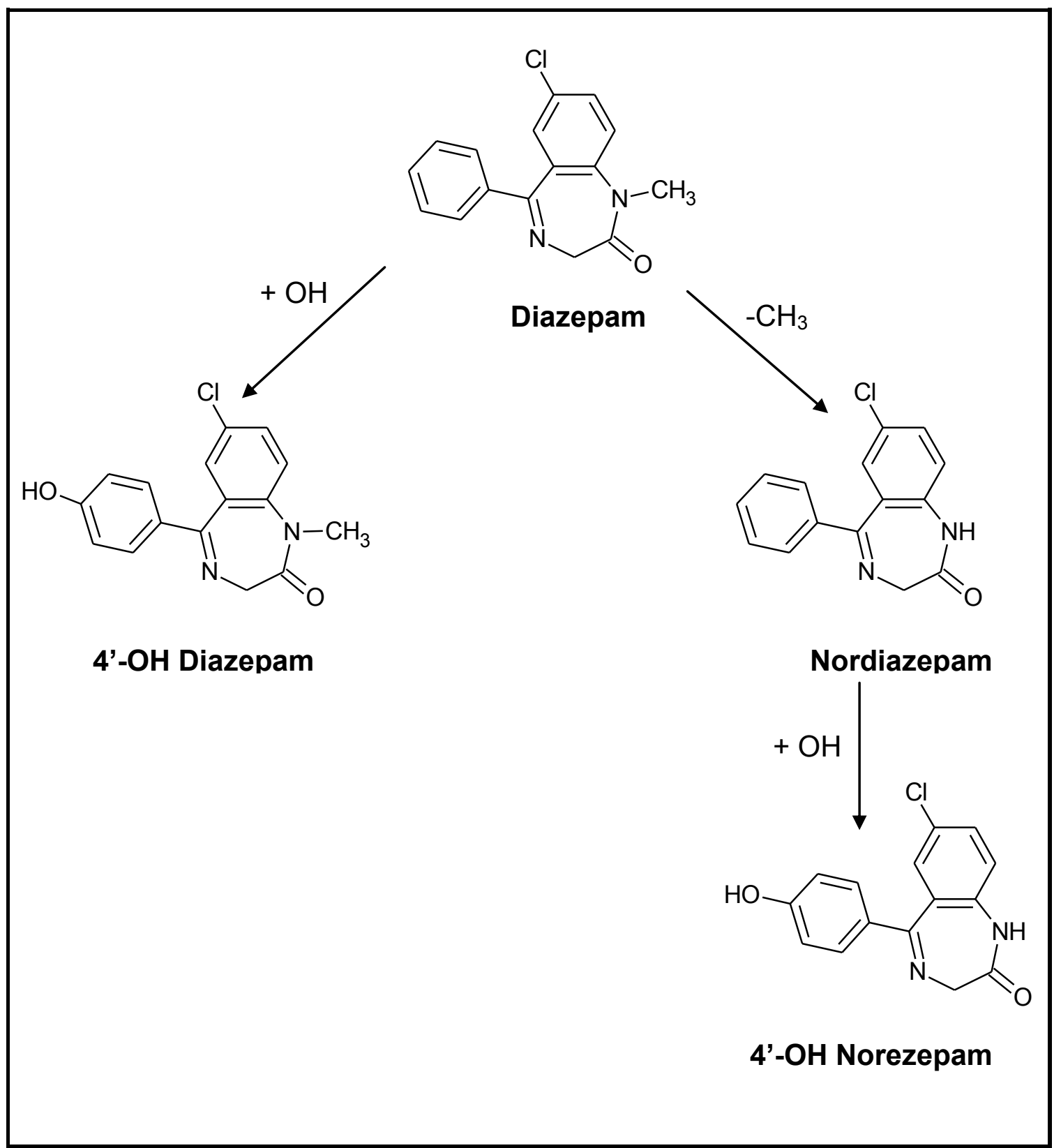

\subsubsection{Effects of E.coli on DZ}

RCM samples prepared for biotransformation studies were spiked with an analyte of interest at $1000 \mathrm{ng} / \mathrm{mL}$ as well as either E. coli, B. fragilis, C. perfringens, or a mixture of the three. Each sample was prepared in duplicate and analyzed in triplicate. 
Pre- and post-incubation aliquots were taken for analysis and the change in concentration results were calculated.

The RCM in DZ biotransformation studies inoculated with E. coli demonstrated the marked turbidity indicative of prominent bacterial growth. As discussed in Section 2.8.0, a $250 \mu \mathrm{L}$ aliquot of $E$. coli resulted in approximately $2.0 \times 10^{8} \mathrm{CFU} / \mathrm{mL}$ after anaerobic incubation, which was comparable to estimates of bacterial enumeration in the human ileum and colon. The OD and turbidity of RCM supported that viable E. coli, those capable of growth, division, and metabolic activity, were present in the biotransformation study samples.

Analysis of the effects of $E$. coli on DZ concentration demonstrated an average change in concentration of $-28.1 \pm 2.7 \mathrm{ng} / \mathrm{mL}$ in sample 1 and $-40.0 \pm 3.0 \mathrm{ng} / \mathrm{mL}$ in sample 2. These results were summarized in Table 25 and Table 26, respectively.

Table 25 - Pre- and Post-Incubation Analysis: Effects of E. coli on Diazepam 1

\begin{tabular}{|ccccc|}
\hline & \multicolumn{4}{c|}{ Concentration $(\mathbf{n g} / \mathbf{m L})$} \\
\hline Sample & DZ & NDZ & OX & TZ \\
\hline Pre E.coli 1 & $191.8 \pm 2.1$ & $0.1 \pm 0.0$ & $0.0 \pm 0.0$ & $0.0 \pm 0.0$ \\
\hline Post E.coli 1 & $163.7 \pm 1.5$ & $0.0 \pm 0.0$ & $0.0 \pm 0.0$ & $0.0 \pm 0.0$ \\
\hline$\Delta$ Concentration & $-28.1 \pm 2.7$ & $0.0 \pm 0.0$ & $0.0 \pm 0.0$ & $0.0 \pm 0.0$ \\
\hline
\end{tabular}


Table 26 - Pre- and Post-Incubation Analysis: Effects of E. coli on Diazepam 2

\begin{tabular}{|ccccc|}
\hline & \multicolumn{4}{c|}{ Concentration $(\mathbf{n g} / \mathbf{m L})$} \\
\hline Sample & DZ & NDZ & OX & TZ \\
\hline Pre E.coli 2 & $197.9 \pm 1.6$ & $0.1 \pm 0.0$ & $0.0 \pm 0.0$ & $0.0 \pm 0.0$ \\
\hline Post E.coli 2 & $157.9 \pm 1.7$ & $0.0 \pm 0.0$ & $0.0 \pm 0.7$ & $0.0 \pm 0.0$ \\
\hline$\Delta$ Concentration & $-40.0 \pm 3.0$ & $0.1 \pm 0.0$ & $0.4 \pm 0.7$ & $0.0 \pm 0.0$ \\
\hline
\end{tabular}

As seen in the drug blank samples, neither NDZ, OX, nor TZ registered a detectible or quantifiable increase from pre- to post-incubation concentrations. In sample 2, instrumental response did indicate a decrease in ND concentration of $0.1 \pm$ $0.0 \mathrm{ng} / \mathrm{mL}$, and an increase in OX concentration of $0.4 \pm 0.7 \mathrm{ng} / \mathrm{mL}$ between pre- and post-incubation samples. However, the ion ratios for NDZ and OX were not within the acceptable range of $\pm 20 \%$ as defined in Table 17 . The experimental values were also below the LOD and LOQ values established in the method validation (Section 3.3.7, Table 18) for both analytes. Without fulfillment of these components along with RT and peak shape, an analyte cannot be detected or confirmed with certainty. Therefore, the changes in concentration for NDZ and OX were insignificant.

The average change in concentration between pre- and post-incubation DZ blanks ranged from -11.1 to $-21.4 \mathrm{ng} / \mathrm{mL}$ while samples incubated with $E$. coli ranged from -28.1 to $-40.0 \mathrm{ng} / \mathrm{mL}$. The discrepancies between blank and biotransformation study samples, though slight, indicated that there may be additional factors contributing to the transformation of DZ when incubated with E.coli. While the majority of DZ disappearance could be attributed to the proposed unassisted degradation, the mass balance of the reaction suggested that DZ was being converted to a compound other than the human metabolites NDZ, OX, and TZ monitored. In this case, E. coli 
possessed a specific enzyme or combination of enzymes that were capable of cleaving

DZ to form some type of alternative minor metabolite.

\subsubsection{Effects of $B$. fragilis on DZ}

Turbidity in $B$. fragilis samples was comparable to that seen in the bacterial blank and E. coli biotransformation incubations. Analysis of the effects of $B$. fragilis on $D Z$ demonstrated an average change in DZ concentration between pre- and postincubation of $-46.2 \pm 2.2 \mathrm{ng} / \mathrm{mL}$ in sample 1 and $-23.9 \pm 3.5 \mathrm{ng} / \mathrm{mL}$ in sample 2 . These results were summarized in Table 27 and Table 28, respectively.

Table 27 - Pre- and Post-Incubation Analysis: Effects of B. fragilis on Diazepam 1

\begin{tabular}{|ccccc|}
\hline & \multicolumn{4}{c|}{ Concentration $(\mathrm{ng} / \mathrm{mL})$} \\
\hline Sample & DZ & NDZ & OX & TZ \\
\hline Pre B. fragilis 1 & $179.7 \pm 1.5$ & $0.1 \pm 0.0$ & $0.0 \pm 0.0$ & $0.0 \pm 0.0$ \\
\hline Post B. fragilis 1 & $133.5 \pm 0.8$ & $0.0 \pm 0.0$ & $0.0 \pm 0.0$ & $0.0 \pm 0.0$ \\
\hline$\Delta$ Concentration & $-46.2 \pm 2.2$ & $-0.1 \pm 0.0$ & $0.0 \pm 0.0$ & $0.0 \pm 0.0$ \\
\hline
\end{tabular}

Table 28 - Pre- and Post-Incubation Analysis: Effects of $B$. fragilis on Diazepam 2

\begin{tabular}{|ccccc|}
\hline & \multicolumn{4}{c|}{ Concentration $(\mathbf{n g} / \mathbf{m L})$} \\
\hline Sample & DZ & NDZ & OX & TZ \\
\hline Pre B. fragilis 2 & $172.5 \pm 3.3$ & $0.1 \pm 0.0$ & $0.0 \pm 0.1$ & $0.0 \pm 0.0$ \\
\hline Post B. fragilis 2 & $148.6 \pm 0.9$ & $0.1 \pm 0.0$ & $0.0 \pm 0.0$ & $0.0 \pm 0.0$ \\
\hline$\Delta$ Concentration & $-23.9 \pm 3.5$ & $0.0 \pm 0.0$ & $0.0 \pm 0.0$ & $0.0 \pm 0.0$ \\
\hline
\end{tabular}

In agreement with the analyte blank and biotransformation studies with E. coli, the mass balance of DZ was affected; however, NDZ, OX, nor TZ registered a response greater than their respective LOD or LOQ in incubations with $B$. fragilis. As suggested with the E. coli studies, the majority of DZ disappearance could be attributed to the proposed unassisted degradation. But the change in DZ concentration is greater than 
that observed in the analyte blanks. This may have resulted from an enzymatic reaction utilized by $B$. fragilis to cleave $D Z$ and produce an alternative metabolite. It is unclear whether E.coli and B. fragilis employed the same enzyme to account for the discrepancy between the drug blank and bacterially inoculated samples or if the DZ cleavage was attributed to species-specific enzymes.

The proposed alternative metabolites could be added to the LC-MS/MS method and monitored for changes between pre- and post-incubation analysis. A kinetic study on the rate of disappearance of $\mathrm{DZ}$ might also provide valuable information that could aid in compound identification. Additionally, analysis of the post-incubation sample via LC-MS/MS in full scan mode might offer some information about the $\mathrm{m} / \mathrm{z}$ of the unidentified compound.

\subsubsection{Effects of $C$. perfringens on DZ}

The effects of $C$. perfringens on DZ produced marked turbidity and a concentration difference of $-0.7 \pm 2.7 \mathrm{ng} / \mathrm{mL}$ in sample 1 and $-1.5 \pm 1.0 \mathrm{ng} / \mathrm{mL}$ in sample 2. Results were summarized in Table 29 and Table 30.

Table 29 - Pre- and Post-Incubation Analysis: Effects of C. perfringens on Diazepam 1

\begin{tabular}{|ccccc|}
\hline & \multicolumn{4}{c|}{ Concentration $(\mathbf{n g} / \mathbf{m L})$} \\
\hline Sample & $\mathbf{D Z}$ & NDZ & OX & TZ \\
\hline Pre C. perfringens 1 & $172.5 \pm 1.5$ & $0.1 \pm 0.0$ & $0.0 \pm 0.0$ & $0.0 \pm 0.0$ \\
\hline Post C. perfringens 1 & $171.9 \pm 1.3$ & $0.1 \pm 0.0$ & $0.0 \pm 0.0$ & $0.0 \pm 0.0$ \\
\hline$\Delta$ Concentration & $-0.7 \pm 2.7$ & $0.0 \pm 0.0$ & $0.0 \pm 0.0$ & $0.0 \pm 0.0$ \\
\hline
\end{tabular}


Table 30 - Pre- and Post-Incubation Analysis: Effects of C. perfringens on Diazepam 2

\begin{tabular}{|ccccc|}
\hline & \multicolumn{4}{c|}{ Concentration $\mathbf{( n g / m L})$} \\
\hline Sample & $\mathbf{D Z}$ & $\mathbf{N D Z}$ & $\mathbf{O X}$ & $\mathbf{T Z}$ \\
\hline Pre C. perfringens 2 & $184.9 \pm 0.5$ & $0.1 \pm 0.0$ & $0.0 \pm 0.0$ & $0.0 \pm 0.0$ \\
\hline Post C. perfringens 2 & $183.5 \pm 0.5$ & $0.1 \pm 0.0$ & $0.0 \pm 0.0$ & $0.0 \pm 0.0$ \\
\hline$\Delta$ Concentration & $-1.5 \pm 1.0$ & $0.0 \pm 0.0$ & $0.0 \pm 0.0$ & $0.0 \pm 0.0$ \\
\hline
\end{tabular}

Similar to the studies performed on $E$. coli and $B$. fragilis, there were negligible changes in concentration detected for NDZ, OX, and TZ. However, there was minimal change in DZ concentration as well. These findings supported the theory that effects of E. coli and B. fragilis resulted in the discrepancies between DZ analyte blanks and their respective biotransformation studies.

The small change in DZ concentration, however, is unique. Disappearance of DZ concentration similar to that seen in the analyte blanks was expected. However, this was not observed in the DZ samples inoculated with C. perfringens. The concentration difference between pre- and post-incubation was small enough that it could potentially be explained by differences in instrumental response. Because DZ experienced this uniquely minimal concentration loss, it is possible that introduction of $C$. perfringens or perhaps a bacterial secretion somehow stabilized the analyte and prevented conversion or degradation.

\subsubsection{Effects of a Mixed Culture on DZ}

The effects of joint incubation with E. coli, B. fragilis, and C. perfringens on DZ produced turbid samples with a concentration difference of $-1.1 \pm 1.8 \mathrm{ng} / \mathrm{mL}$ in sample 1 and $-4.8 \pm 0.7 \mathrm{ng} / \mathrm{mL}$ in sample 2. Results were summarized in Table 31 and Table 32 . 
Table 31 - Pre- and Post-Incubation Analysis: Effects of Mixed Culture on Diazepam 1

\begin{tabular}{|ccccc|}
\hline & \multicolumn{4}{c|}{ Concentration $(\mathbf{n g} / \mathbf{m L})$} \\
\hline Sample & DZ & NDZ & OX & TZ \\
\hline Pre Mixed Culture 1 & $140.0 \pm 0.7$ & $0.1 \pm 0.0$ & $0.0 \pm 0.0$ & $0.0 \pm 0.0$ \\
\hline Post Mixed Culture 1 & $138.8 \pm 1.4$ & $0.1 \pm 0.0$ & $0.0 \pm 0.0$ & $0.0 \pm 0.0$ \\
\hline$\Delta$ Concentration & $-1.1 \pm 1.8$ & $0.0 \pm 0.0$ & $0.0 \pm 0.0$ & $0.0 \pm 0.0$ \\
\hline
\end{tabular}

Table 32 - Pre- and Post-Incubation Analysis: Effects of Mixed Culture on Diazepam 2

\begin{tabular}{|ccccc|}
\hline & \multicolumn{4}{c|}{ Concentration $(\mathbf{n g} / \mathbf{m L})$} \\
\hline Sample & $\mathbf{D Z}$ & NDZ & OX & TZ \\
\hline Pre Mixed Culture 2 & $159.3 \pm 1.1$ & $0.1 \pm 0.0$ & $0.0 \pm 0.0$ & $0.0 \pm 0.0$ \\
\hline Post Mixed Culture 2 & $154.5 \pm 1.5$ & $0.1 \pm 0.0$ & $0.0 \pm 0.0$ & $0.0 \pm 0.0$ \\
\hline$\Delta$ Concentration & $-4.8 \pm 0.7$ & $0.0 \pm 0.0$ & $0.0 \pm 0.0$ & $0.0 \pm 0.0$ \\
\hline
\end{tabular}

Samples were incubated with $100 \mu \mathrm{L}$ each of $E$. coli and B. fragilis, and $C$. perfringens. As seen with the C. perfringens samples, there was minimal change in DZ concentration. This supported the supposition that the presence of $C$. perfringens stabilized the analyte and prevented unassisted degradation as well as the proposed conversions by $E$. coli and $B$. fragilis. As expected, there were negligible changes in concentration detected for NDZ, OX, and TZ.

\subsubsection{Flunitrazepam Blanks}

Analysis of the FLU drug blanks exhibited a decrease in concentration of the target analyte similar to that seen with DZ. FLU Drug Blank 1 (Table 33) produced an average change in concentration of $-25.9 \pm 6.3 \mathrm{ng} / \mathrm{mL}$ and FLU Drug Blank 2 (Table 34) an average difference of $-40.2 \pm 0.8 \mathrm{ng} / \mathrm{mL}$. Unlike what was observed in the DZ blank experiments, however, there was a corresponding increase in concentration for FLU metabolites in the post-incubation samples. The 7AM metabolite exhibited increases in 
concentration of $15.7 \pm 0.1 \mathrm{ng} / \mathrm{mL}$ (FLU Drug Blank 1) and $15.1 \pm 0.2 \mathrm{ng} / \mathrm{mL}$ (FLU Drug Blank 2).

Table 33 - Pre- and Post-Incubation Analysis: Flunitrazepam Drug Blank 1

\begin{tabular}{|cccc|}
\hline & \multicolumn{3}{c|}{ Concentration (ng/mL) } \\
\hline Sample & FLU & 7AM & NDES \\
\hline Pre FLU Blank 1 & $182.7 \pm 5.3$ & $0.1 \pm 0.0$ & $0.0 \pm 0.0$ \\
\hline Post FLU Blank 1 & $156.8 \pm 2.1$ & $15.9 \pm 0.1$ & $0.0 \pm 0.0$ \\
\hline$\Delta$ Concentration Average & $-25.9 \pm 6.3$ & $+15.7 \pm 0.1$ & $0.0 \pm 0.0$ \\
\hline
\end{tabular}

Table 34 - Pre- and Post-Incubation Analysis: Flunitrazepam Drug Blank 2

\begin{tabular}{|cccc|}
\hline & \multicolumn{3}{c|}{ Concentration $(\mathbf{n g} / \mathrm{mL})$} \\
\hline Sample & FLU & 7AM & NDES \\
\hline Pre FLU Blank 2 & $186.6 \pm 3.7$ & $0.2 \pm 0.0$ & $0.0 \pm 0.0$ \\
\hline Post FLU Blank 2 & $146.4 \pm 2.9$ & $15.3 \pm 0.2$ & $0.0 \pm 0.0$ \\
\hline$\Delta$ Concentration Average & $-40.2 \pm 0.8$ & $+15.1 \pm 0.2$ & $0.0 \pm 0.0$ \\
\hline
\end{tabular}

FLU Drug Blank samples maintained sterility throughout the duration of the experiments. Mass balance of the reaction indicated that approximately half of the decrease in FLU concentration between pre- and post-incubation samples was contributed to conversion to metabolite 7AM. This was most likely in response to the incubation conditions and duration which led to an unassisted degradation. These responses were supported by the processed sample stability study performed in the $D Z$ and FLU method validation (Section 3.3.8). In the stability study conducted for the 500 $\mathrm{ng} / \mathrm{mL}$ sample, FLU demonstrated an average $-54.1 \mathrm{ng} / \mathrm{mL}$ in concentration while $7 \mathrm{AM}$ produced an average of $+11.5 \mathrm{ng} / \mathrm{mL}$ from $\mathrm{T}=0$ to $\mathrm{T}=48$ hours. A visual representation of pre- and post-incubation analyte concentrations was summarized in Figure 21. 
Figure 21 - Pre and Post-Incubation Concentrations of Flunitrazepam and Metabolites

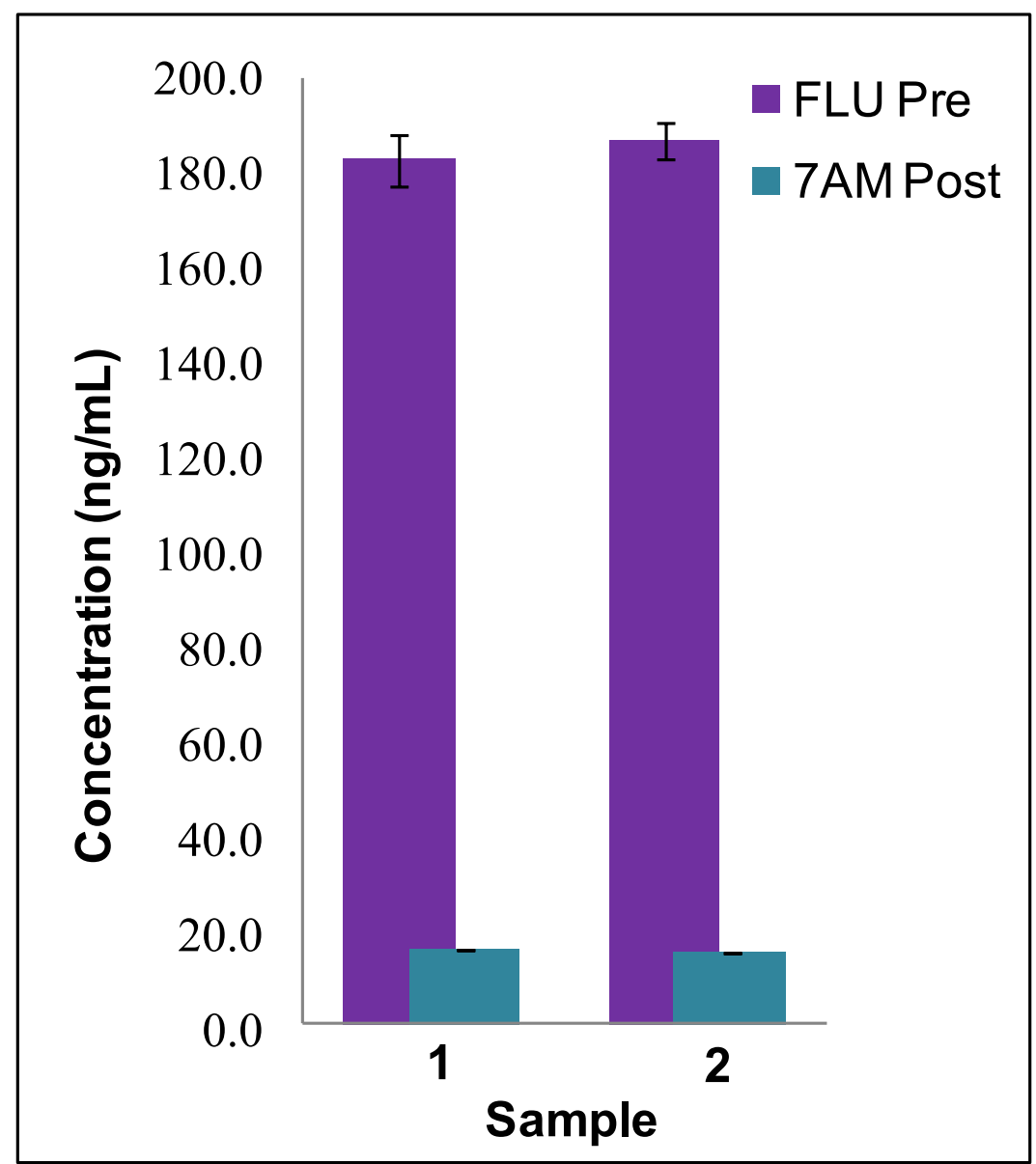

The remaining mass balance discrepancy, $-8.3 \mathrm{ng} / \mathrm{mL}$ in sample 1 and -23.3 $\mathrm{ng} / \mathrm{mL}$ in sample 2, between pre- and post-incubation FLU in the analyte blank concentrations was otherwise unaccounted for. Mass balance for the reaction was calculated by converting the original FLU concentration to moles and subtracting the number of moles of 7AM produced. The remainder was reconverted back to grams of FLU. This was the most accurate way to account for differences in mass between loss of FLU and production of 7AM. NDES was absent in both pre- and post incubation blanks. This suggested that FLU likely performed a partial, unassisted degradation to a product other than the monitored metabolites. 


\subsubsection{Effects of E.coli on FLU}

Analysis of the effects of $E$. coli on FLU concentration resulted in an average concentration difference of $-112.4 \pm 2.6 \mathrm{ng} / \mathrm{mL}$ in sample 1 and $-142.0 \pm 3.3 \mathrm{ng} / \mathrm{mL}$ in sample 2. These results were summarized in Tables 35 and Table 36, respectively.

Table 35 - Pre- and Post-Incubation Analysis: Effects of E. coli on Flunitrazepam 1

\begin{tabular}{|cccc|}
\hline & \multicolumn{3}{c|}{ Concentration $(\mathrm{ng} / \mathrm{mL})$} \\
\hline Sample & FLU & 7AM & NDES \\
\hline Pre E.coli 1 & $142.0 \pm 1.8$ & $0.1 \pm 0.0$ & $0.0 \pm 0.0$ \\
\hline Post E.coli 1 & $29.6 \pm 0.9$ & $93.5 \pm 1.3$ & $0.0 \pm 0.0$ \\
\hline$\Delta$ Concentration Average & $-112.4 \pm 2.6$ & $+93.4 \pm 1.3$ & $0.0 \pm 0.0$ \\
\hline
\end{tabular}

Table 36 - Pre- and Post-Incubation Analysis: Effects of E. coli on Flunitrazepam 2

\begin{tabular}{|cccc|}
\hline & \multicolumn{3}{c|}{ Concentration $(\mathrm{ng} / \mathrm{mL})$} \\
\hline Sample & FLU & 7AM & NDES \\
\hline Pre E.coli Blank 2 & $175.5 \pm 3.6$ & $0.2 \pm 0.0$ & $0.0 \pm 0.0$ \\
\hline Post E.coli Blank 2 & $33.4 \pm 0.3$ & $15.3 \pm 0.6$ & $0.0 \pm 0.0$ \\
\hline$\Delta$ Concentration Average & $-142.0 \pm 3.3$ & $+93.7 \pm 0.6$ & $0.0 \pm 0.0$ \\
\hline
\end{tabular}

As seen in the FLU drug blank samples, 7AM registered an increase in postincubation samples. However, the conversion of FLU to 7AM occurred to a greater extent in samples inoculated with E. coli. Of the $112.4 \mathrm{ng} / \mathrm{mL}$ of FLU converted, an average of $93.4 \mathrm{ng} / \mathrm{mL}$ was recovered in the form of 7AM in sample 1. Mass balance of the reaction indicated that approximately $9.0 \mathrm{ng} / \mathrm{mL}$ of the original FLU concentration was unaccounted for in sample 1 and $38.4 \mathrm{ng} / \mathrm{mL}$ in sample 2 . Of the $142.0 \mathrm{ng} / \mathrm{mL}$ of FLU converted in sample 2 , only $93.7 \mathrm{ng} / \mathrm{mL}$ was converted to $7 \mathrm{AM}$. The results can be seen in Figure 22. 
Figure 22 - Effects of E. coli on Pre and Post-Incubation Concentrations of Flunitrazepam and Metabolites

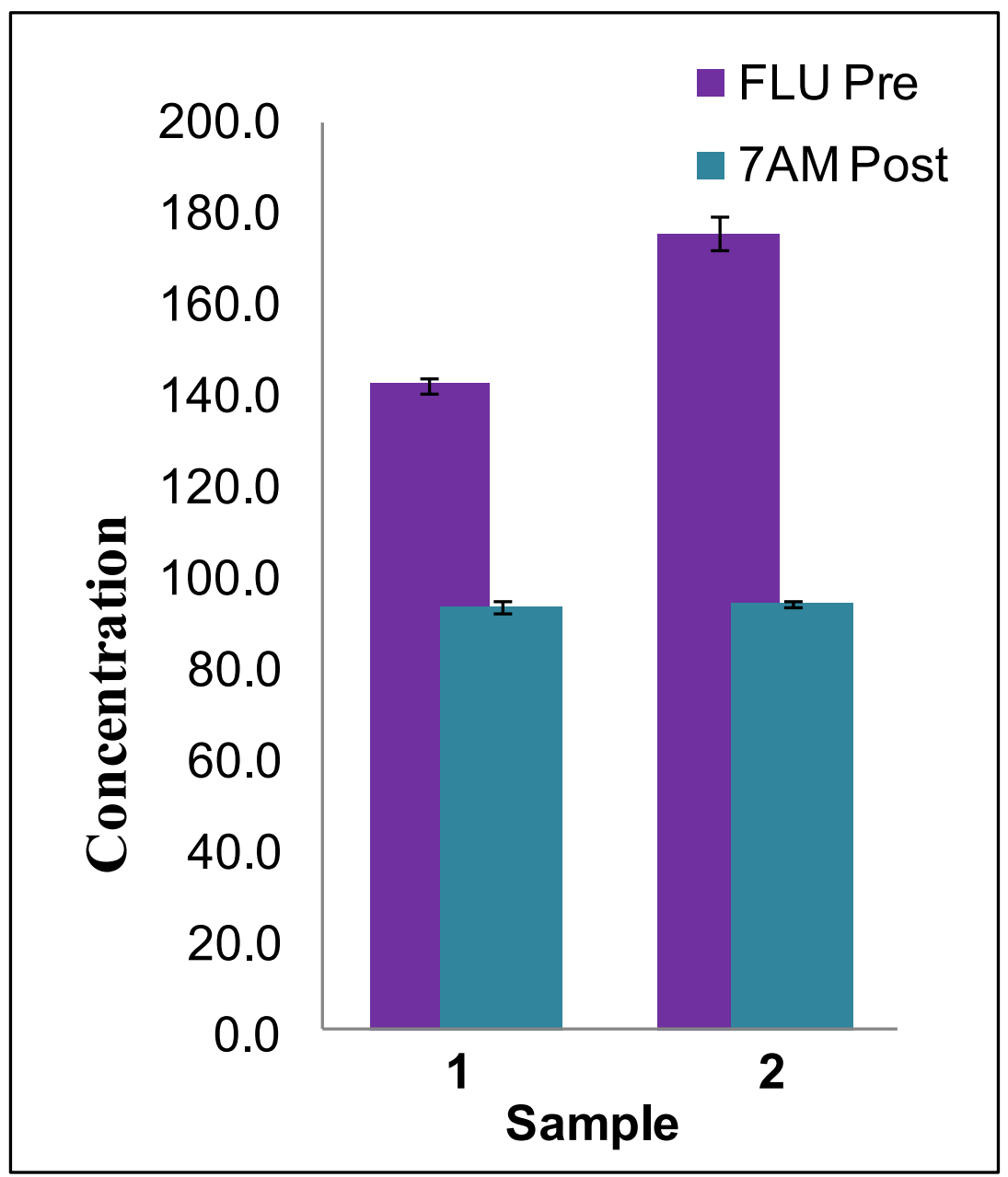

In the FLU drug blanks, $15.1-15.7 \mathrm{ng} / \mathrm{mL}$ of $7 \mathrm{AM}$ was recovered from the postincubation samples. This was similar to the conversion seen in sample 2. Both samples demonstrated similar OD values and turbidity. This supported the theory that FLU was being converted to an additional metabolite not monitored in this analysis. There were several possible explanations for the discrepancies between FLU disappearance and the appearance of 7AM. Based on the extent of FLU conversion, it was evident that 7AM was the major metabolite produced by the biotransformation conducted by E. coli in both samples. However, discrepancies in the mass balance of 
the reaction indicated that these bacterial species produced an alternative metabolite or metabolites as well.

There were several possible explanations for the discrepancies between FLU disappearance and the appearance of 7AM. 3-hydroxyflunitrazepam was a potential candidate that should be included in future analysis and is depicted in Figure 23.

Figure 23 - Conversion of Flunitrazepam to 3-hydroxyflunitrazepam<smiles>CN1C(=O)CN=C(c2ccccc2F)c2cc([N+](=O)[O-])ccc21</smiles>

FLU

3-hydroxyflunitrazepam

Additionally, Malanciuc et al. published a metabolism of FLU scheme that included several additional compounds. ${ }^{58}$ These compounds were not included in this research because 7-acetamino-flunitrazepam and 7-amino-nor-flunitrazepam are not directly FLU metabolites. They are produced by the conversion of 7AM and can be seen in Figure 24. Either of these were potential minor metabolites that would have explained the slight concentration differences between the disappearance of FLU and the production of 7AM. 
Figure 24 - Conversion of 7-Aminoflunitrazepam to 7-Acetamino-Flunitrazepam and 7Amino-Nor-Flunitrazepam

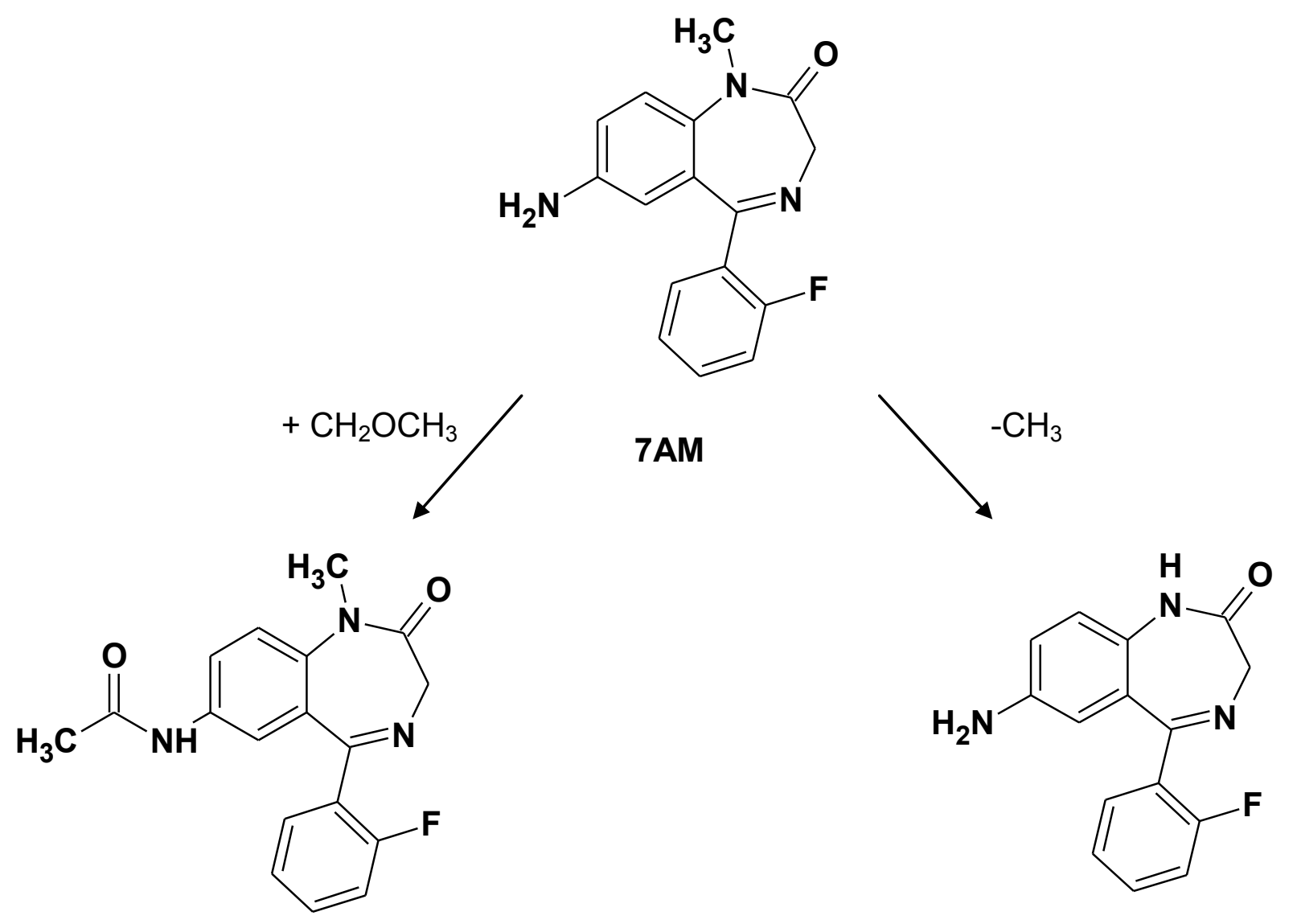

7-acetamino-flunitrazepam

7-amino-nor-flunitrazepam

As suggested with DZ, additional FLU and 7AM metabolites may account for the discrepancies in mass balance of the reaction. Kinetic studies on the rate of disappearance of FLU and rate of appearance of 7AM might also provide valuable information that could aid in unidentified minor metabolite identification. Additionally, analysis of the post-incubation sample via LC-MS/MS in full scan mode might offer some information concerning the $\mathrm{m} / \mathrm{z}$ of the unidentified compound. 


\subsubsection{Effects of $B$. fragilis on FLU}

Analysis of the effects of $B$. fragilis on FLU concentration produced turbid samples and demonstrated an average concentration difference of $-151.5 \pm 2.7 \mathrm{ng} / \mathrm{mL}$ in sample 1 and $-117.9 \pm 3.2 \mathrm{ng} / \mathrm{mL}$ in sample 2 . In both instances, this represented complete degradation of FLU. These results were summarized in Table 37 and Table 38 , respectively.

Table 37 - Pre- and Post-Incubation Analysis: Effects of $B$. fragilis on Flunitrazepam 1

\begin{tabular}{|cccc|}
\hline & \multicolumn{3}{c|}{ Concentration $(\mathrm{ng} / \mathrm{mL})$} \\
\hline Sample & FLU & 7AM & NDES \\
\hline Pre B. fragilis 1 & $151.5 \pm 2.7$ & $9.7 \pm 0.1$ & $0.0 \pm 0.0$ \\
\hline Post B. fragilis 1 & $0.0 \pm 0.0$ & $129.3 \pm 0.6$ & $0.0 \pm 0.0$ \\
\hline$\Delta$ Concentration Average & $-151.5 \pm 2.7$ & $+119.6 \pm 0.7$ & $0.0 \pm 0.0$ \\
\hline
\end{tabular}

Table 38 - Pre- and Post-Incubation Analysis: Effects of $B$. fragilis on Flunitrazepam 2

\begin{tabular}{|cccc|}
\hline & \multicolumn{3}{c|}{ Concentration $(\mathrm{ng} / \mathrm{mL})$} \\
\hline Sample & FLU & 7AM & NDES \\
\hline Pre B. fragilis 2 & $117.9 \pm 3.2$ & $8.6 \pm 0.1$ & $0.0 \pm 0.0$ \\
\hline Post B. fragilis 2 & $0.0 \pm 0.0$ & $103.3 \pm 0.9$ & $0.0 \pm 0.0$ \\
\hline$\Delta$ Concentration Average & $-117.9 \pm 3.2$ & $+94.8 \pm 0.9$ & $0.0 \pm 0.0$ \\
\hline
\end{tabular}

In sample 1, the 7AM concentration increased by $+119.6 \mathrm{ng} / \mathrm{mL}$. Mass balance of the reaction suggested that approximately $8.6 \mathrm{ng} / \mathrm{mL}$ of the original FLU was converted to a compound other than those that were monitored in this method. Approximately 3.7 $\mathrm{ng} / \mathrm{mL}$ of initial FLU was unaccounted for in sample 2. These effects can be seen in Figure 25. 
Figure 25 - Effects of $B$. fragilis on Pre and Post-Incubation Concentrations of Flunitrazepam and Metabolites

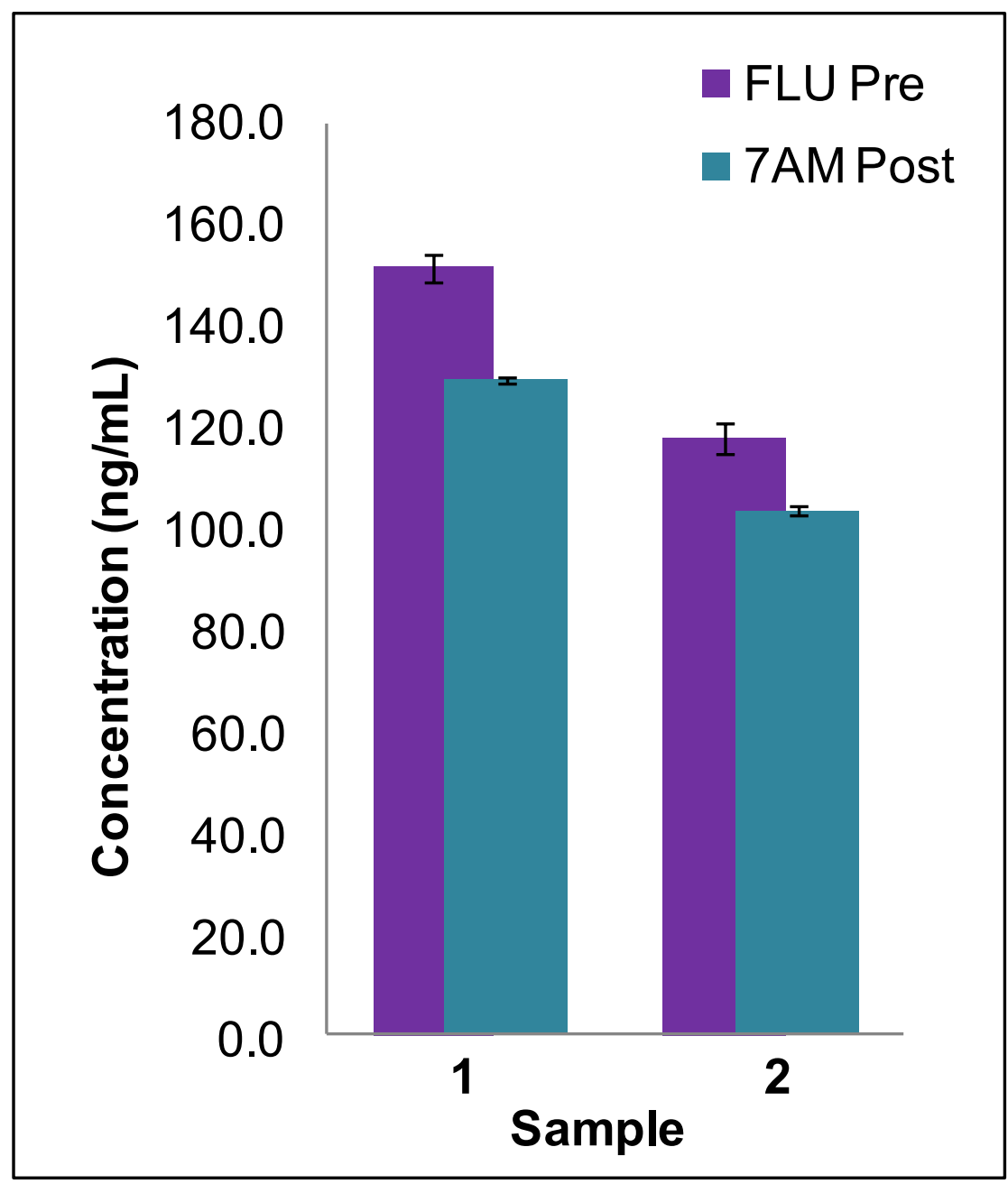

\subsubsection{Effects of $C$. perfringens on FLU}

The effects of $C$. perfringens on FLU were similar to those produced by $B$. fragilis. Incubation with C. perfringens resulted in complete reduction of FLU between pre- and post-incubation samples for a total of $-164.5 \pm 4.7 \mathrm{ng} / \mathrm{mL}$ in sample 1 and $137.2 \pm 2.1 \mathrm{ng} / \mathrm{mL}$ in sample 2. Results were summarized in Table 39 and Table 40 . 
Table 39 - Pre- and Post-Incubation Analysis: Effects of $C$. perfringens on Flunitrazepam 1

\begin{tabular}{|cccc|}
\hline & \multicolumn{3}{c|}{ Concentration $(\mathrm{ng} / \mathrm{mL})$} \\
\hline Sample & FLU & 7AM & NDES \\
\hline Pre C. perfringens 1 & $164.5 \pm 4.7$ & $0.1 \pm 0.0$ & $0.0 \pm 0.0$ \\
\hline Post C. perfringens 1 & $0.0 \pm 0.0$ & $134.0 \pm 0.9$ & $0.0 \pm 0.0$ \\
\hline$\Delta$ Concentration Average & $-164.5 \pm 4.7$ & $+133.8 \pm 0.9$ & $0.0 \pm 0.0$ \\
\hline
\end{tabular}

Table 40 - Pre- and Post-Incubation Analysis: Effects of $C$. perfringens on Flunitrazepam 2

\begin{tabular}{|c|c|c|c|}
\hline & \multicolumn{3}{|c|}{ Concentration (ng/mL) } \\
\hline Sample & FLU & 7AM & NDES \\
\hline Pre C. perfringens 2 & $137.2 \pm 2.1$ & $0.1 \pm 0.0$ & $0.0 \pm 0.0$ \\
\hline Post C. perfringens 2 & $0.0 \pm 0.0$ & $124.0 \pm 0.7$ & $0.0 \pm 0.0$ \\
\hline$\Delta$ Concentration Average & $-137.2 \pm 2.1$ & $+123.9 \pm 0.7$ & $\begin{array}{cc}0.0 & 0.0\end{array}$ \\
\hline
\end{tabular}

In sample 1 , the 7 AM concentration increased by $+133.8 \mathrm{ng} / \mathrm{mL}$. Mass balance of the reaction suggested that approximately $16.4 \mathrm{ng} / \mathrm{mL}$ of FLU was converted to an alternative compound or metabolite. In sample 2 , only $0.1 \mathrm{ng} / \mathrm{mL}$ of the original FLU concentration was unrecovered. These effects were depicted in Figure 26. The potential minor metabolites proposed for $E$. coli and B. fragilis biotransformations were also candidates with C. perfringens inoculates, however, further investigations would be required before a definitive identification could be provided. 
Figure 26 - Effects of C. perfringens on Pre and Post-Incubation Concentrations of Flunitrazepam and Metabolites

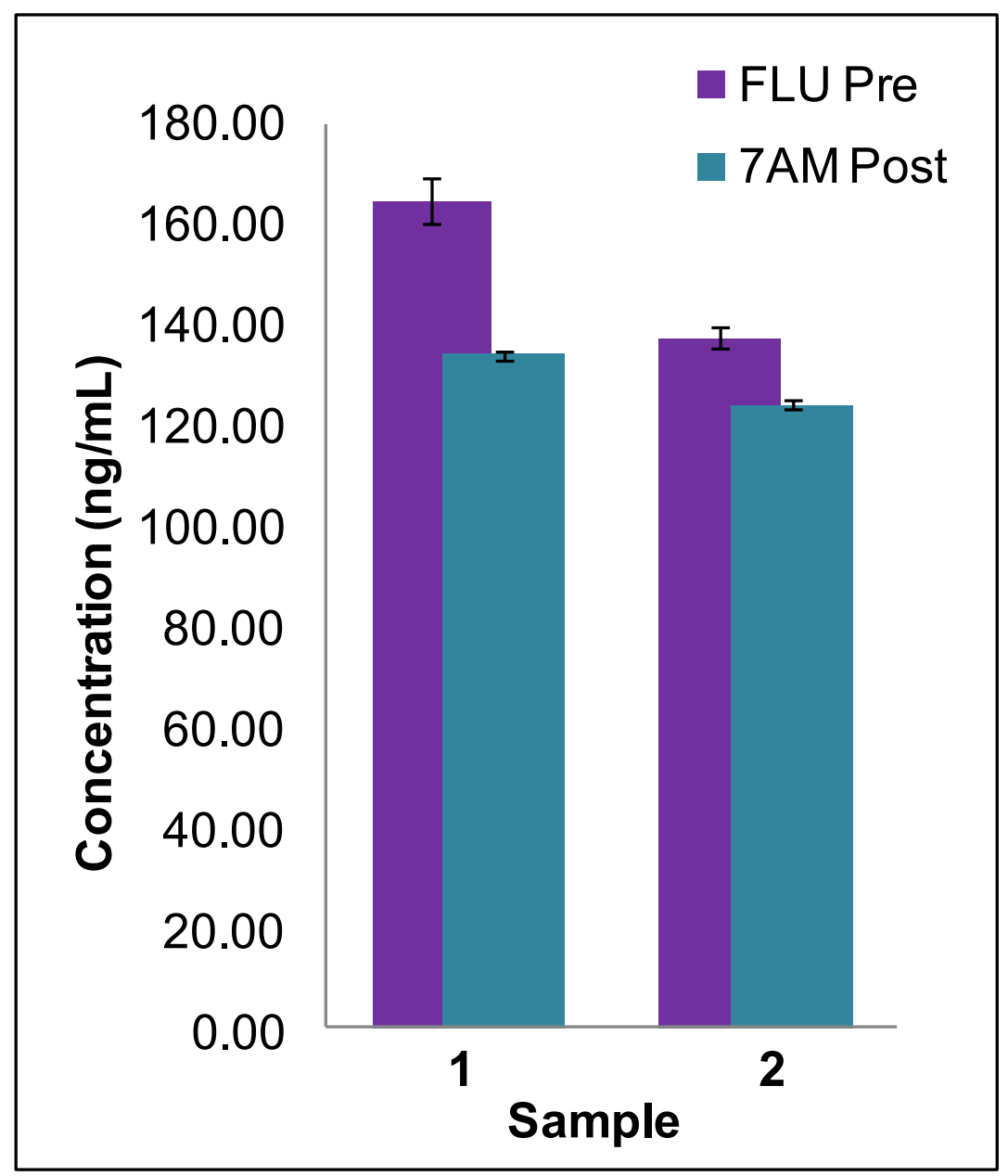

\subsubsection{Effects of a Mixed Culture on FLU}

The effects of the mixed culture reduced the initial FLU concentrations by -103.3 $\pm 2.6 \mathrm{ng} / \mathrm{mL}$ in sample 1 and $-149.6 \pm 2.0 \mathrm{ng} / \mathrm{mL}$ in sample 2 , both of which represented complete conversions. Results were summarized in Tables 41 and 42. 
Table 41 - Pre- and Post-Incubation Analysis: Effects of Mixed Culture on Flunitrazepam 1

\begin{tabular}{|cccc|}
\hline & \multicolumn{3}{c|}{ Concentration $(\mathrm{ng} / \mathrm{mL})$} \\
\hline Sample & FLU & 7AM & NDES \\
\hline Pre Mixed Culture 1 & $103.3 \pm 2.6$ & $3.5 \pm 0.1$ & $0.0 \pm 0.0$ \\
\hline Post Mixed Culture 1 & $0.0 \pm 0.0$ & $86.9 \pm 1.2$ & $0.0 \pm 0.0$ \\
\hline$\Delta$ Concentration Average & $-103.3 \pm 2.6$ & $+83.4 \pm 1.2$ & $0.0 \pm 0.0$ \\
\hline
\end{tabular}

Table 42 - Pre- and Post-Incubation Analysis: Effects of C. perfringens on Flunitrazepam 2

\begin{tabular}{|cccc|}
\hline & \multicolumn{3}{c|}{ Concentration $(\mathbf{n g} / \mathrm{mL})$} \\
\hline Sample & FLU & 7AM & NDES \\
\hline Pre Mixed Culture 2 & $150.9 \pm 2.9$ & $3.9 \pm 0.0$ & $0.0 \pm 0.0$ \\
\hline Post Mixed Culture 2 & $0.0 \pm 0.0$ & $123.7 \pm 0.0$ & $0.0 \pm 0.0$ \\
\hline$\Delta$ Concentration Average & $-149.6 \pm 2.0$ & $+119.9 \pm 0.0$ & $0.0 \pm 0.0$ \\
\hline
\end{tabular}

FLU demonstrated concentration loss for each bacterial species individually as well as the mixture of bacteria. Mixed culture samples produced the complete transformation of FLU as seen with $B$. fragilis and C. perfringens. Mass balance of the reaction demonstrated that approximately $7.2 \mathrm{ng} / \mathrm{mL}$ of FLU in sample 1 and 14.1 $\mathrm{ng} / \mathrm{mL}$ in sample 2 was not recovered in the form of 7AM. Data are depicted in Figure 27. As with the other cultures, data suggested that an alternative metabolite or multiple metabolites were likely being produced. However, the identity of these structures will require further experiments and analysis. 
Figure 27 - Effects of Mixed Culture on Pre and Post-Incubation Concentrations of Flunitrazepam and Metabolites

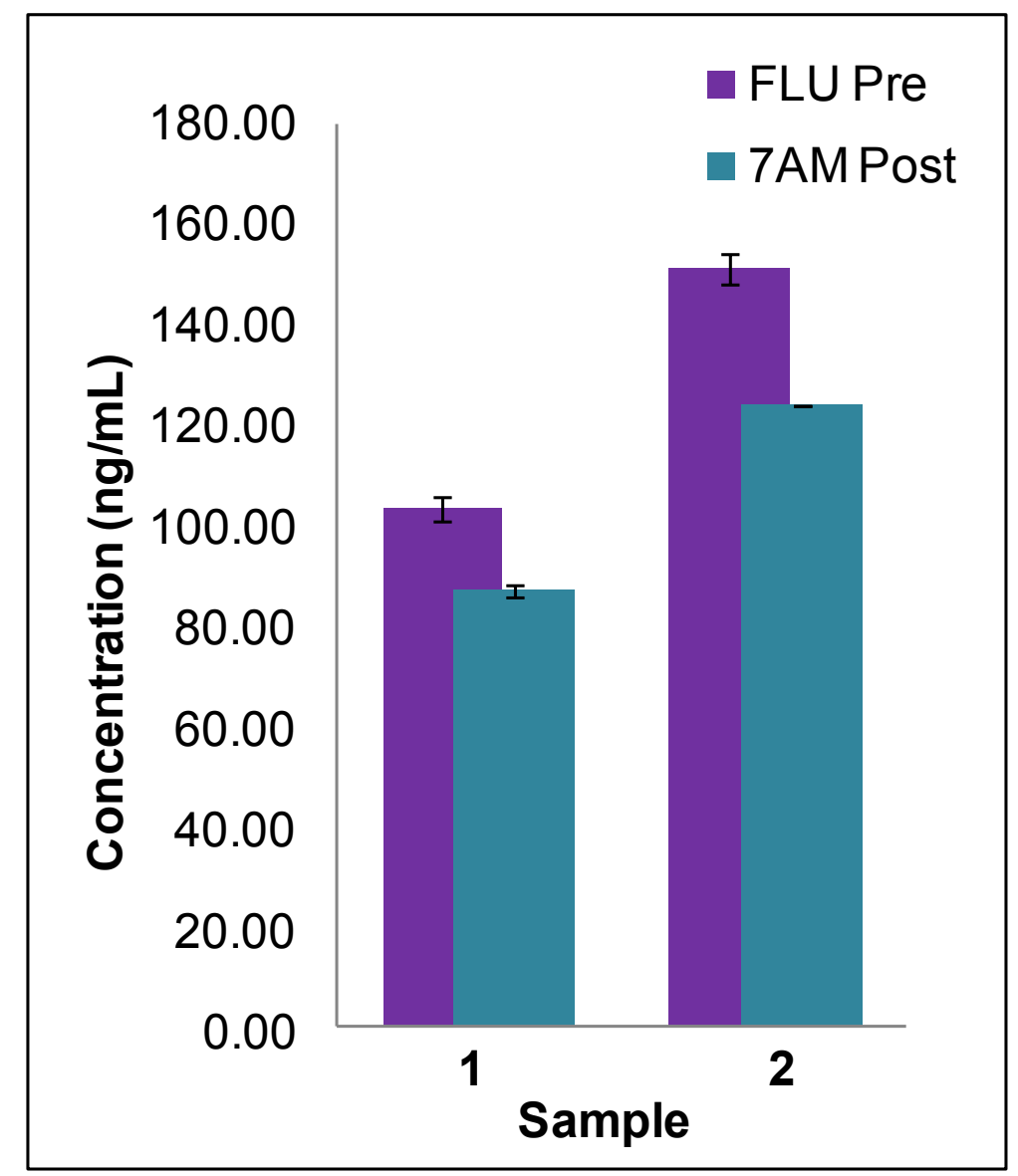

The data exhibited by the effects of E. coli, B. fragilis, C. perfringens, and mixed culture was consistent with that published by Robert and Drummer, detailed in section 1.1.3. ${ }^{16}$ In this study, Robertson and Drummer studied effects of $8 \mathrm{GI}$ bacteria on clonazepam, flunitrazepam, and nitrazepam in blood. They found that all three compounds were converted to their respective 7 -amino metabolites, though the rate of conversion varied amongst the different species. However, analysis was limited by their methodology. The experimental run time was 25 minutes whereas the previously validated LC-MS/MS method was 8 minutes. LOQ values for their analytes were all 10 $\mathrm{ng} / \mathrm{mL}$. LOQ values for the presented methods ranged from $0.1-1.0 \mathrm{ng} / \mathrm{mL}$ with $\mathrm{DZ}$ and 
FLU at 0.5 and $7 A M$ at $0.1 \mathrm{ng} / \mathrm{mL}$. In addition, they did not prepare analyte blanks for analysis. As seen in this research, DZ and FLU both underwent a partial, unassisted degradation when incubation under experimental conditions and duration. These authors also performed temperature studies to study the effect of temperature on conversion rate of FLU to 7AM. This would be an interesting inclusion in future works.

\subsubsection{Conclusions}

An LC-MS/MS method was previously validated for the simultaneous detection, identification, and quantitation of the benzodiazepines DZ, FLU, and their respective metabolites which were recovered from RCM using SPE (Chapter 3). The method was applied to experimental explorations involving bacterial biotransformation of the drugs of abuse DZ and FLU.

Pre- and post-incubation aliquots were extracted and analyzed via LC-MS/MS. Analytes and metabolites were evaluated for acceptable peak shape, retention time, ion ratios, and $\mathrm{S} / \mathrm{N} \geq 3$ for detection as well as bias and precision within $\pm 20 \%$ for

quantitation. A series of blanks was prepared in RCM and incubated alongside samples inoculated with bacteria.

Analysis and mass balance of the DZ blank suggested the analyte must undergo a partial, unassisted degradation when incubated at $37^{\circ} \mathrm{C}$ for 48 hours. However, NDZ, OX, nor TZ registered an increase from pre- to post-incubation concentrations. The DZ degradation product was therefore unidentified. Analysis of the FLU drug blanks likewise exhibited a decrease in concentration; however, there was a corresponding 
increase in concentration in 7AM metabolites in the post-incubation samples that accounted for some of the FLU loss.

Analysis of the effects of $E$. coli and $B$. fragilis on $\mathrm{DZ}$ concentration demonstrated an average concentration difference greater than that seen in the blanks. In accordance with drug blank samples, neither NDZ, OX, nor TZ registered a detectible or quantifiable increase from pre- to post-incubation concentrations. The discrepancies between blank and biotransformation study samples indicated that there may be additional factors contributing to the transformation of DZ when incubated with E.coli or B. fragilis. A possible explanation was that DZ was being converted to a compound other than the human metabolites NDZ, OX, and TZ monitored. In this case, E. coli and B. fragilis possessed a specific enzyme or combination of enzymes that were capable of cleaving DZ to form some type of alternative metabolite. Additional experimental and literature review may offer some guidance.

There were negligible changes in concentration detected for NDZ, OX, and TZ in samples inoculated with C. perfringens. However, there was minimal change in DZ concentration as well. These findings supported the theory of $E$. coli and $B$. fragilis were responsible for the concentration discrepancies between the DZ drug blanks and their respective biotransformation studies. The small change in DZ concentration, however, was unique. The difference was small enough that it could potentially be explained by differences in instrumental response. However, the C. perfringens samples were incubated alongside the other blanks and inoculates, and loss of DZ concentration similar to that seen in the drug blank samples was expected. Since DZ experienced minimal concentration loss, it is possible that introduction of $C$. perfringens 
stabilized the analyte and prevented degradation. The effects of mixed culture resulted in similar responses to those seen in C. perfringens. This supported the supposition that the presence of $C$. perfringens stabilized the analyte and prevented the proposed degradation by $E$. coli and $B$. fragilis.

E. coli, B. fragilis, C. perfringens, and a mixture of the three species were all capable of transforming FLU to 7AM to varying extents in RCM. E. coli converted the majority of FLU but was not as efficient as the anaerobic species. 7AM exhibited an increase in concentration between pre- and post-incubation samples which accounted for some of the FLU loss. However, not all of the original FLU concentration was recovered as 7AM as evidenced by mass balance of the reaction. This could have been attributed to transformation of FLU into an additional metabolite that was not monitored such as 3-hydroxyflunitrazepam. Another possibility was conversion of 7AM to 7-acetamino-flunitrazepam and/or 7-amino-nor-flunitrazepam. However, further analysis would be required to determine the presence and identity of these or other potentially unique bacterial metabolites. 


\section{Chapter 5 - A Validated Method for the Quantitation of Cocaine, Fentanyl, and Metabolites in Reinforced Clostridial Medium by Liquid Chromatography-Tandem Mass Spectrometry}

\subsubsection{Introduction}

In Chapter 3, a method was developed and validated for the quantitation of DZ and FLU in RCM. This chapter focused on expanding the previous methodology to investigate other analytes of interest.

A novel LC-MS/MS method was developed and validated for simultaneous analysis and quantitation of cocaine $(\mathrm{COC})$ and metabolites anhydroecgonine methyl ester (AEME), benzoylecgonine (BZE), cocaethylene (CE), ecgonine methyl ester (EME), m-hydroxycocaine ( $\mathrm{mHC}$ ), and norcocaine (NC) as well as fentanyl (FENT), and metabolites norfentanyl (NF), and despropionyl fentanyl (DESP) in RCM. RCM is a bacterial nutrient medium, the components of which were discussed in Table 2.

COC and FENT are classified by the DEA as Schedule II drugs of abuse. ${ }^{24}$ They were selected as the target analytes due to the importance in analytical and forensic toxicology. Human metabolic studies have been well documented and the analyte and metabolite standards were commercially available.

According to the Substance Abuse and Mental Health Services Administration (SAMHSA) - National Survey on Drug Use and Health there were approximately 1.1 million COC users in the United States in $2012 .{ }^{64}$ COC is most commonly snorted, smoked, or injected, and acts as a CNS stimulant. It is considered to be highly addictive compound and is linked to adverse health effects including increase in blood pressure, body temperature, and heart rate, as well as feelings of panic and paranoia. 
$\mathrm{COC}$ has been known to cause cardiovascular complications, seizures, and stroke in users as well. Human metabolism of COC has been extensively studied, and many metabolites have been identified. Only the first generation metabolites of AEME, BZE, $\mathrm{CE}, \mathrm{EME}, \mathrm{mHC}$, and $\mathrm{NC}$ were investigated for the purposes of this research. The metabolism scheme for COC is displayed in Figure $28 .^{50,51,65-82}$

Figure 28 - Human Metabolites of Cocaine

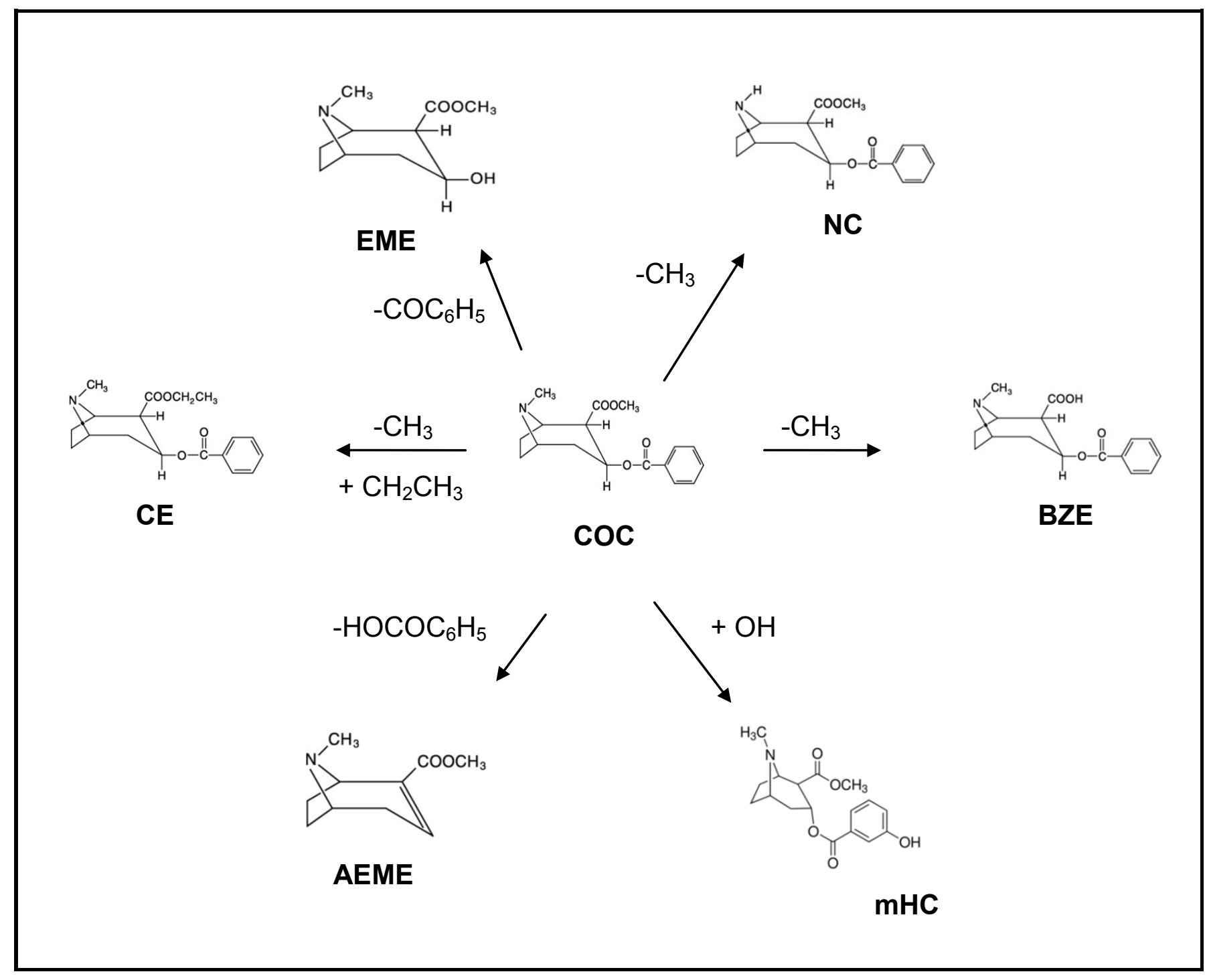


FENT is a synthetic opiod analgesic. It is classified as both a drug for medicinal purposes and a drug of abuse. For medical purposes, it is known as Actiq ${ }^{\circledR}$, Duragesic $®$, and Sublimaze ${ }^{2}{ }^{24,64,82}$ It is most often used in various forms of pain management. This drug is commonly administered as an injection, but can also be seen in transdermal patches as well as lozenges. It binds to opiate receptors in the body and causes nausea, sedation, respiratory depression, respiratory arrest, and coma. $^{24,64,82}$ Human metabolites include NFENT and DESP. The metabolism scheme for FENT is displayed in Figure 29. ${ }^{51,84-88}$

Figure 29 - Human Metabolites of Fentanyl

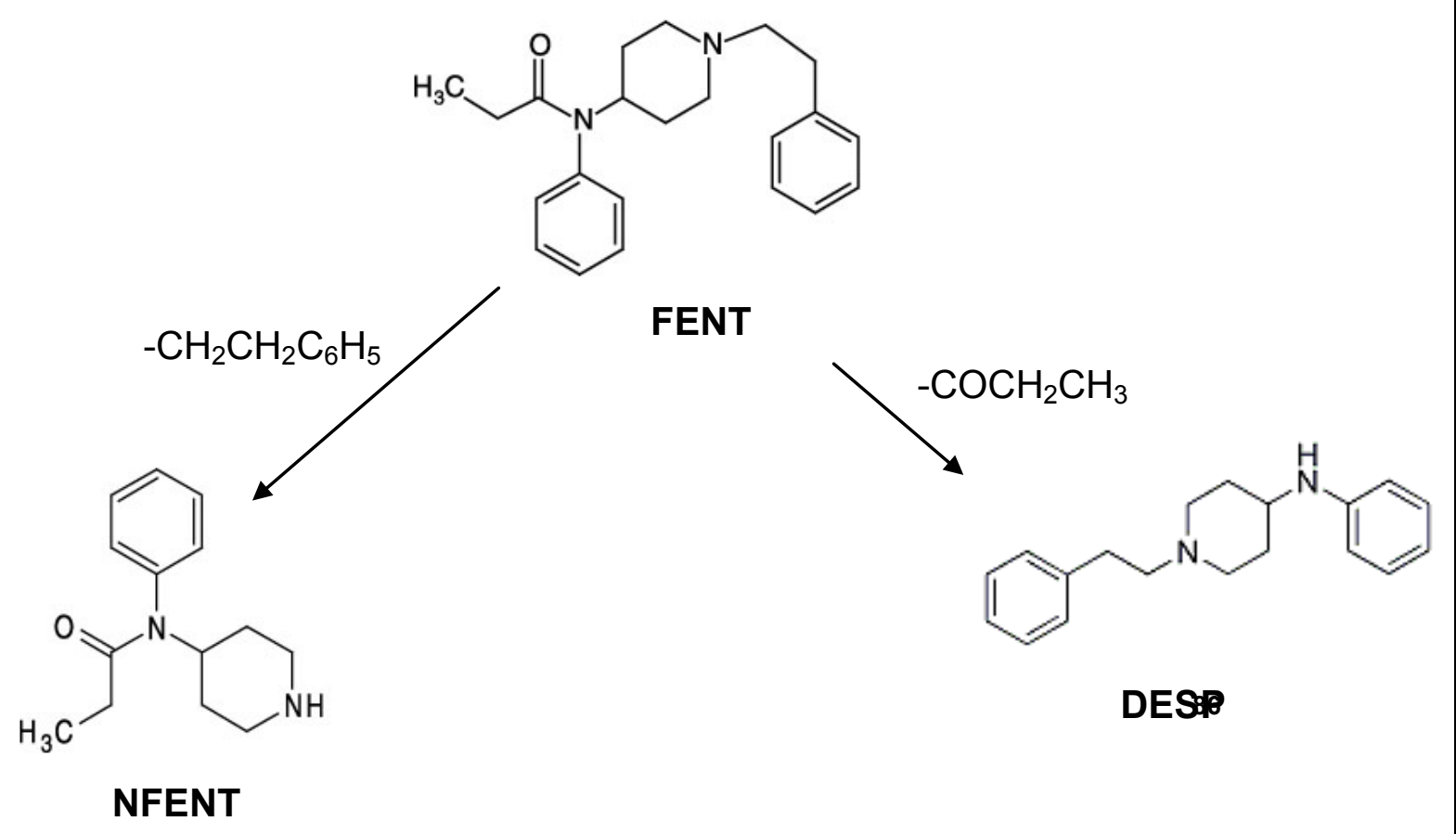




\subsubsection{Materials and Methods}

\subsubsection{Materials}

Analyte reference standards for COC, AEME, BZE, CE, EME, NC, FENT, and NFENT as $1.0 \mathrm{mg} / \mathrm{mL}$, and cocaine-d3 (COC-d3), benzoylecgonine-d3 (BZE-d3), cocaethylene-d3 (CE-d3), ecgonine methyl ester-d3 (EME-d3), norcocaine-d3 (NC-d3), fentanyl-d5 (FENT-d5), and norfentanyl-d5 (NFENT-d5) as $100 \mu \mathrm{g} / \mathrm{mL}$ were purchased from Cerilliant $₫$ (Round Rock, TX). The m-hydroxycocaine $(\mathrm{mHC})$ was purchased as a $1.0 \mathrm{mg}$ solid from Sigma-Aldrich ${ }^{\circledR}$ (St. Louis, MO) and despropionyl-3-methylfentanyl (despropionyl fentanyl) (DESP) was purchased as a $1.0 \mathrm{mg}$ solid from Enzo ${ }^{\circledR}$ Life Sciences (Farmingdale, NY). Both of these standards were dissolved in $1 \mathrm{~mL}$ of $\mathrm{MeOH}$ for working standard and dilution preparations.

Ammonium acetate, ammonium hydroxide $\left(\mathrm{NH}_{4} \mathrm{OH}\right)$, FA eluent additive for $\mathrm{LC}$ MS, hydrochloric acid $(\mathrm{HCl})$, and LC-MS CHROMASOLV® $A C N$ and $\mathrm{MeOH}$ were purchased from Sigma-Aldrich ${ }^{\circledR}$. The IPA (Optima ${ }^{\mathrm{TM}} \mathrm{LC} / \mathrm{MS}$ ) was purchased from Fischer Scientific and HPLC grade EA from JT Baker ${ }^{\circledR}$ (Center Valley, PA). Strata ${ }^{\mathrm{TM}}$-XDrug B Strong Cation Mixed Mode $60 \mathrm{mg} / 3 \mathrm{~mL}$ cartridges were obtained from Phenomenex® (Torrance, CA). The narrow bore ZORBAX Eclipse XDB-C8 (2.1 mm x $150 \mathrm{~mm} \times 5 \mu \mathrm{m})$ column affixed with a ZORBAX Eclipse XDB-C8 $(2.1 \mathrm{~mm} \times 12.5 \mathrm{~mm} \times$ $5 \mu \mathrm{m}$ ) guard cartridge were obtained from Agilent Technologies (Santa Clara, CA). RCM was obtained from BD Difco ${ }^{\mathrm{TM}}$ (Sparks, MD). 


\subsubsection{Sample Preparation}

An initial calibration curve was prepared from working solutions in buffer and plotted at $0.05,0.1,0.25,0.5,1,5,10,25,50,100$, and $200 \mathrm{ng} / \mathrm{mL}$. Calibration curves for each analyte in buffer can be seen in Appendix D. Three different SPE methods were evaluated for recovery of COC, FENT, and metabolites. Reagents and solvents consisted of acetonitrile (ACN), ethyl acetate (EA), isopropyl alcohol (IPA), formic acid (FA), methanol (MeOH), and ammonium hydroxide $\left(\mathrm{NH}_{4} \mathrm{OH}\right)$. Details of the methods are shown in Figure 30 and Figure 31.

Figure 30 - SPE 1 and SPE 2 for Cocaine, Fentanyl and Metabolites

\begin{tabular}{|c|c|c|}
\hline Cartr & $\begin{array}{l}\text { SPE } 1 \\
\text { dge: Strata-X-Drug B }\end{array}$ & $\begin{array}{c}\text { SPE } 2 \\
\text { Cartridge: Strata-X-Drug B }\end{array}$ \\
\hline Wash & $\begin{array}{l}\cdot 0.1 \mathrm{~N} \mathrm{HCl} \\
\cdot \mathrm{MeOH}\end{array}$ & $\begin{array}{ll} & \cdot 0.1 \% \mathrm{FA} \\
\text { Wash } & \cdot \mathrm{MeOH} / \mathrm{H}_{2} \mathrm{O}(30 / 70 \mathrm{v} / \mathrm{v})\end{array}$ \\
\hline Dry & -10 minutes & - 10 minutes \\
\hline Elute & $\begin{array}{l}\cdot \text { EA/IPA/NH }{ }_{4} \mathrm{OH} \\
(70 / 20 / 10 \mathrm{v} / \mathrm{v})\end{array}$ & $\begin{array}{l}\cdot 2 \% \mathrm{NH}_{4} \mathrm{OH} \text { in } \\
\text { ACN/MeOH }(1 / 1 \mathrm{v} / \mathrm{v})\end{array}$ \\
\hline
\end{tabular}


Figure 31 - SPE 3 for Cocaine, Fentanyl and Metabolites

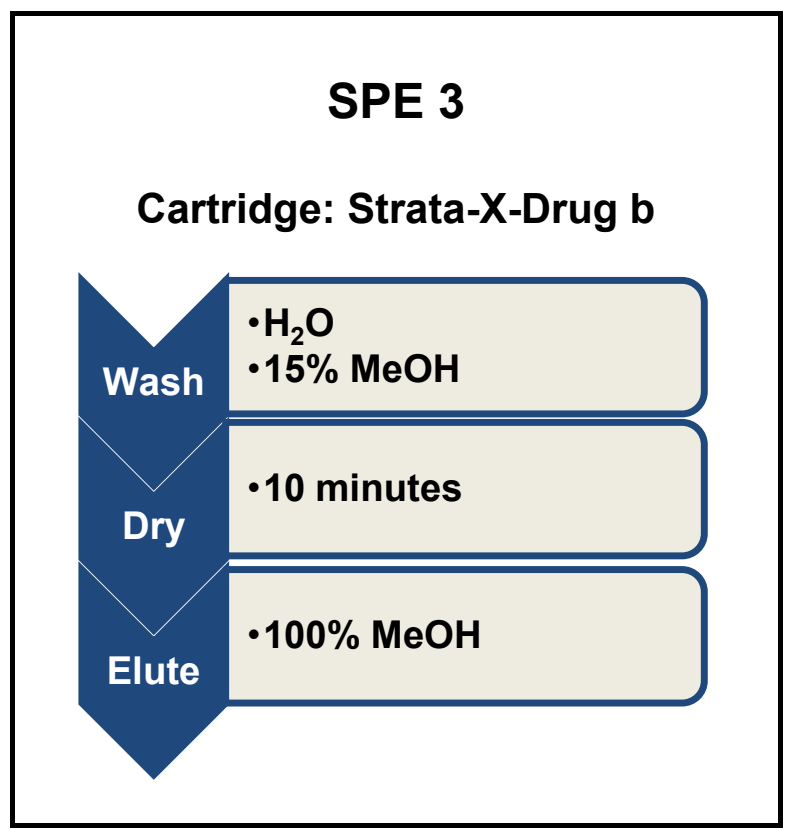

Three working solutions containing all ten analytes at 10,000 ng/mL (WS1), 1000 $\mathrm{ng} / \mathrm{mL}$ (WS2), and $10 \mathrm{ng} / \mathrm{mL}$ (WS3) in water/acetonitrile:methanol (80/20 v/v) were used to prepare the dilution sets required in the method validation studies. An internal standard working solution consisting of the seven deuterated standard compounds was prepared at $2500 \mathrm{ng} / \mathrm{mL}$. The RCM matrix was composed of ten $100 \mathrm{~mL}$ aliquots of $\mathrm{RCM}$ (3.8 $\mathrm{g} \mathrm{RCM}$ in $100 \mathrm{~mL}$ of DI water) which were prepared, pooled, and then autoclaved at $121^{\circ} \mathrm{C}$ for 15 minutes.

All samples were spiked with $200 \mu \mathrm{L}$ of $0.1 \mathrm{~N} \mathrm{HCl}$ to ensure $\mathrm{pH}$ between 4.0 and 6.0 which was necessary for extraction. SPE was used to isolate target analytes from media components. Extractions were performed at 3 concentrations spanning the concentration range. After analysis, SPE 1 was chosen for this method. 
The Strata ${ }^{\mathrm{TM}}-\mathrm{X}$-Drug $\mathrm{B}$ cartridges are based on a polymeric strong-cation mixed mode sorbent specifically designed for basic drugs of abuse. These cartridges and SPE 1 method were chosen because they demonstrated the greatest responses for the analytes of interest and metabolites across the three concentrations. The cartridges did not require conditioning steps which was advantageous because SPE required less time and solvent per sample. This also reduced the amount of hazardous waste produced as well.

Samples $\left(1 \mathrm{~mL}\right.$ ) were directly loaded onto the Strata ${ }^{\mathrm{TM}}$-X-Drug B cartridges (no conditioning) and washed with $1 \mathrm{~mL}$ of $0.1 \mathrm{~N} \mathrm{HCl}$. Wash 1 was designed to minimize salt content and other polar compounds that could interfere with instrumental analysis. Cartridges were also washed with $1 \mathrm{~mL}$ of $\mathrm{MeOH}$ (Wash 2) to minimize the nonpolar matrix constituents, and then dried under full vacuum for 10 minutes. Samples were eluted with $1 \mathrm{~mL}$ of a freshly prepared mixture of EA:IPA: $\mathrm{NH}_{4} \mathrm{OH}(70: 20: 10 \mathrm{v} / \mathrm{v} / \mathrm{v})$, dried under nitrogen, and reconstituted in water/acetonitrile:methanol (80/20 v/v).

\subsubsection{Instrumental Analysis}

Method development and analysis were performed using LC-MS/MS. Instrumentation was the same used to develop and validate the DZ and FLU method in Chapter 3. Samples $(2 \mu \mathrm{L})$ were injected at a flow rate of $0.250 \mathrm{~mL} / \mathrm{min}$. The buffer system consisted of $20 \mathrm{mM}$ ammonium acetate adjusted to $\mathrm{pH} 2.7$ with $\mathrm{FA}(\mathrm{A})$ and acetonitrile/methanol (50/50 v/v) (B). Analytes were separated using a gradient elution program of $20 \%$ B for 2 minutes, to $45 \%$ B over 10.5 minutes, back to original conditions over 0.1 minute, and then column equilibration for 1.4 minutes. Total run 
time was 14 minutes followed by a 2 minute post-run at $20 \%$ B to ensure stable column conditions before the next injection.

The AJS ESI source was operated in the positive mode and parameters were optimized using Source and iFunnel Optimizer with a $100 \mathrm{ng} / \mathrm{mL}$ sample containing all of the target analytes, metabolites, and standards. The gas temperature was optimized at $\left(265^{\circ} \mathrm{C}\right)$, gas flow $(19 \mathrm{~L} / \mathrm{min})$, nebulizer $(50 \mathrm{psi})$, sheath gas temperature $\left(350^{\circ} \mathrm{C}\right)$, sheath gas flow (11 L/min), capillary (3250 V), $\triangle \mathrm{EMV} \mathrm{(200),} \mathrm{fragmentor} \mathrm{(380} \mathrm{V),} \mathrm{and}$ cell acceleration $(2 \mathrm{~V})$. The column was maintained $a 7^{\circ} \mathrm{C}$ and the autosampler at $4^{\circ} \mathrm{C}$.

MassHunter ${ }^{\mathrm{TM}}$ Optimizer software was used to determine the best MRM transitions for each analyte of interest as well as their corresponding deuterated standards. The software provided up to four ion transitions for each analyte, the results for which can be seen in Appendix E. All of the suggested ions were included in the initial calibration. The two ion transitions that demonstrated the greatest signal response while also maintaining stable ion ratios across the calibration range were selected as the quantifier and qualifier ions for each analyte.

MRM data were only acquired in a narrow window based upon the RT of the analyte of interest. Because some of the ions had similar ion transitions, the narrow range of acquisition increased the selectivity for each individual analytes. Samples were quantified by comparing the peak area response ratio of the target analyte to that of the corresponding deuterated standard. RT, quantifier and (qualifier) ion transitions, $\mathrm{CE}$, ion ratios, and deuterated standard selection for each analyte is summarized in Table 43. 
Table 43 - LC-MS/MS Parameters for Cocaine, Fentanyl, and Respective Metabolites in RCM

\begin{tabular}{|c|c|c|c|c|c|c|}
\hline Analyte & $\begin{array}{c}\mathbf{R T} \\
\text { (mins) }\end{array}$ & $\begin{array}{c}Q 1 \\
(m / z)\end{array}$ & $\begin{array}{c}\text { Q3 } \\
(\mathrm{m} / \mathrm{z})\end{array}$ & $\begin{array}{l}\text { CE } \\
\text { (V) }\end{array}$ & $\begin{array}{c}\text { Ion } \\
\text { Ratio }\end{array}$ & ISTD \\
\hline COC & 9.0 & 304.2 & $\begin{array}{c}182.0 \\
(150.0)\end{array}$ & $\begin{array}{l}13 \\
20\end{array}$ & 8.7 & COC-d3 \\
\hline COC-d3 & 9.0 & 307.2 & 185.0 & 15 & & \\
\hline AEME & 2.05 & 182.1 & $\begin{array}{c}118.0 \\
(122.1)\end{array}$ & $\begin{array}{l}25 \\
17\end{array}$ & 72.0 & EME-d3 \\
\hline BZE & 6.0 & 290.1 & $\begin{array}{c}168.0 \\
(104.9)\end{array}$ & $\begin{array}{l}13 \\
20\end{array}$ & 35.8 & BZE-d3 \\
\hline BZE-d3 & 6.0 & 293.1 & 171.0 & 13 & & \\
\hline CE & 11.2 & 318.2 & $\begin{array}{c}196 \\
(150.0)\end{array}$ & $\begin{array}{l}13 \\
20\end{array}$ & 9.4 & CE-d3 \\
\hline CE-d3 & 11.2 & 321.2 & 199.0 & 13 & & \\
\hline EME & 1.6 & 200.1 & $\begin{array}{c}82.1 \\
(150.0)\end{array}$ & $\begin{array}{l}29 \\
20\end{array}$ & 10.5 & EME-d3 \\
\hline EME-d3 & 1.6 & 203.1 & (185.0) & 13 & & \\
\hline $\mathrm{mHC}$ & 5.65 & 320.2 & $\begin{array}{l}182.0 \\
(82.0)\end{array}$ & $\begin{array}{l}13 \\
20\end{array}$ & 19.9 & BZE-d3 \\
\hline NC & 9.7 & 290.1 & $\begin{array}{c}168.0 \\
(136.0)\end{array}$ & $\begin{array}{c}9 \\
17\end{array}$ & 64.8 & NC-d3 \\
\hline NC-d3 & 9.7 & 293.1 & 171.0 & 9 & & \\
\hline FENT & 13.2 & 337.2 & $\begin{array}{l}(105.0) \\
(188.1)\end{array}$ & $\begin{array}{l}45 \\
21\end{array}$ & 84.5 & FENT-d5 \\
\hline FENT-d5 & 13.2 & 342.3 & 188.0 & 19 & & \\
\hline NFENT & 7.0 & 233.2 & $\begin{array}{c}84.0 \\
(93.9)\end{array}$ & $\begin{array}{l}15 \\
31\end{array}$ & 4.2 & NFENT \\
\hline $\begin{array}{l}\text { NFENT- } \\
\text { d5 }\end{array}$ & 7.0 & 238.0 & 84.0 & 15 & & \\
\hline DESP & 12.5 & 281.2 & $\begin{array}{c}105.0 \\
(188.0)\end{array}$ & $\begin{array}{l}31 \\
15\end{array}$ & 73 & FENT-d5 \\
\hline
\end{tabular}


The separation and MRM transitions of the analytes can be seen in the chromatogram in Figure 32. The purpose of chromatographic separation in this method was to minimize the co-elution of target analytes and their respective metabolites. These compounds were similar in structure and many utilized the same ion transitions, which can lead to ionization effects that affect quantitation. Maximizing analyte separation and limiting MRM data acquisition to the narrow window incorporating analyte retention time enhanced the selectivity for each individual analytes.

Figure 32 - MRM of Cocaine, Fentanyl, and Respective Metabolites in RCM

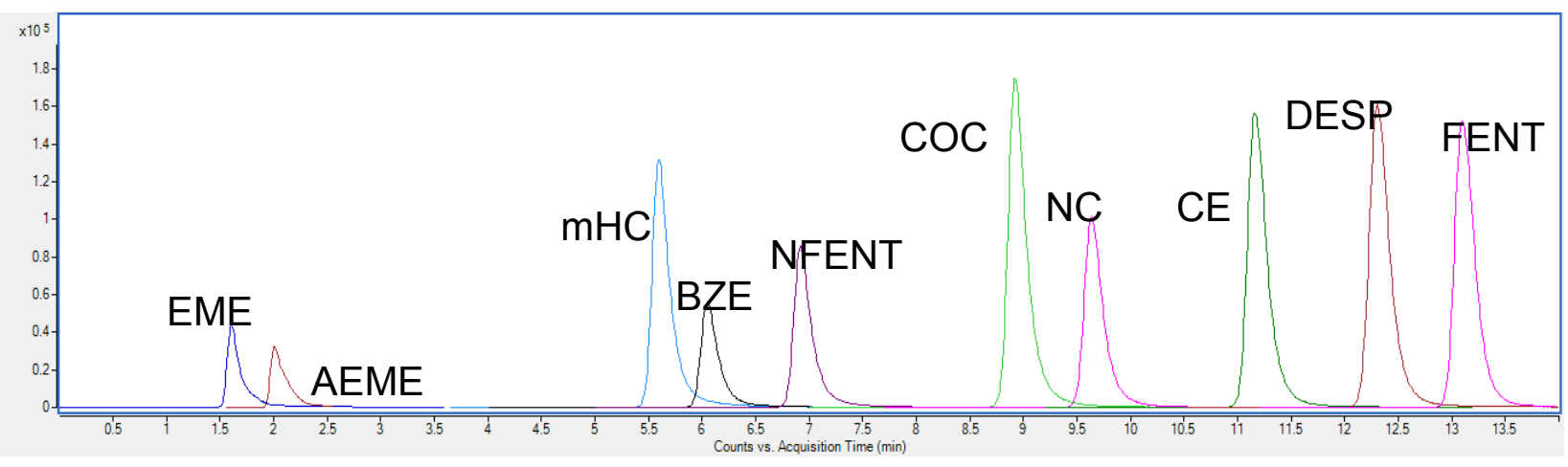

Unlike the DZ and FLU method developed in Chapter 3, deuterated standards were not available for all of the metabolites of interest. Internal standards for these compounds were selected based upon compounds with similar structure as well as RT, a feature of analyte response to the column, buffer system, and elution gradient.

\subsubsection{Method Validation}

Method validation definitions and parameters were detailed in Chapter 1. The presented method was fully validated and included a calibration model, interference studies, carryover effects, ionization suppression and enhancement, ME, RE, PE, bias and precision, LOD, LOQ, dilution integrity, and processed sample stability. 


\subsubsection{Results and Discussion}

\subsubsection{Calibration Model}

Calibration and QC standards were prepared in media using WS1, WS2, and WS3, and the internal standard working solution. Final calibrations were based on a 10point model of $0.1,0.25,0.5,1,5,10,25,50,100$, and $200 \mathrm{ng} / \mathrm{mL}$ for the analytes and $25 \mathrm{ng} / \mathrm{mL}$ for the corresponding deuterated standards. Calibrations were determined using a linear model excluding the origin and with $1 / x$ weighting. Correlation coefficients were $>0.99(n=5)$ for all analytes of interest in RCM, and can be seen in Appendix F. Calibration range, slope, $y$-intercept, $R^{2}, L O D$, and LOQ values are summarized in Table 44 .

Table 44 - Calibration Curve Data, LOD, and LOQ for Cocaine, Fentanyl, and Respective Metabolites in RCM

\begin{tabular}{|ccccccc|}
\hline Analyte & $\begin{array}{c}\text { Calibration Range } \\
(\mathbf{n g} / \mathbf{m L})\end{array}$ & $\begin{array}{c}\text { Slope } \\
\left(\mathbf{x} 10^{-2}\right)\end{array}$ & $\begin{array}{c}\text { Y-Intercept } \\
\left(\mathbf{x} 10^{-3}\right)\end{array}$ & $\mathbf{R}^{2}$ & $\begin{array}{c}\text { LOD } \\
(\mathbf{n g} / \mathbf{m L})\end{array}$ & $\begin{array}{c}\text { LOQ } \\
(\mathbf{n g} / \mathbf{m L})\end{array}$ \\
\hline COC & $0.1-200$ & 4.11 & -5.71 & 0.999 & 0.1 & 0.1 \\
\hline AEME & $10-200$ & 1.31 & 5.29 & 0.995 & 10 & 10 \\
\hline BZE & $0.1-200$ & 4.49 & -0.356 & 0.997 & 0.1 & 0.1 \\
\hline CE & $0.25-200$ & 4.53 & -2.71 & 0.996 & 0.25 & 0.25 \\
\hline EME & $5-200$ & 1.73 & 11.8 & 0.992 & 5.0 & 5.0 \\
\hline mHC & $0.25-200$ & 1.09 & -4.90 & 0.995 & 0.1 & 0.25 \\
\hline NC & $0.25-200$ & 3.87 & 1.09 & 0.996 & 0.1 & 0.25 \\
\hline FENT & $0.25-200$ & 5.70 & 3.43 & 0.995 & 0.1 & 0.25 \\
\hline NFENT & $0.5-200$ & 8.34 & -19.2 & 0.997 & 0.5 & 0.5 \\
\hline DESP & $0.25-200$ & 5.85 & -1.24 & 0.993 & 0.1 & 0.25 \\
\hline
\end{tabular}

\subsubsection{Interference Studies}

Ten RCM samples were prepared, pooled, and then autoclaved for interference and other validation studies. Blank matrix samples were extracted and then analyzed via LC-MS/MS to determine possible interferences with the analytes of interest based 
upon retention time, peak shape, and ion ratio. Some of the analyte responses were indicative of matrix effects, however all of the responses failed to satisfy analyte identification criteria of RT, peak shape, and ion ratios. Extent of matrix effects were examined in greater detail using the Matuszewski methods in Section 5.3.4. ${ }^{28}$

Deuterated standard blanks at $25 \mathrm{ng} / \mathrm{mL}$ were also examined for possible interferences that could be caused by impurities or similar fragmentation patterns of analyte and internal standards. Interferences were negligible based upon the previously established identification criteria.

\subsubsection{Carryover Analysis}

To determine carryover effects, samples were prepared at the two highest calibration points, 100 and $200 \mathrm{ng} / \mathrm{mL}$, as well as two higher concentrations of 300 and $400 \mathrm{ng} / \mathrm{mL}$. Each sample injection was followed by an extracted blank sample injection. Analyte carryover was detected at all four concentrations. To minimize or eliminate these effects, blank samples were injected between each sample analysis.

\subsubsection{Ionization Suppression/Enhancement and Recovery}

ME, RE, and PE were all calculated using the Matuszewski equations and methods which are defined in Section 1.5.3. Three sets of samples were prepared at three concentrations to span the range of LOD and LOQ values of the analytes of interest. ${ }^{28}$ Results were summarized in Table 45 and Table 46. 
Table 45 - Matrix Effects, Recovery, and Process Efficiency for Cocaine and Metabolites in RCM

\begin{tabular}{|c|c|c|c|c|c|c|c|}
\hline Analyte & COC & AEME & BZE & CE & EME & $\mathrm{mHC}$ & NC \\
\hline & \multicolumn{7}{|c|}{ High $(200 \mathrm{ng} / \mathrm{mL})$} \\
\hline ME (\%) & 103.0 & 49.4 & 107.1 & 87.9 & 87.5 & 104.7 & 96.7 \\
\hline RE (\%) & 97.7 & 136.1 & 97.6 & 97.9 & 98.9 & 105.7 & 98.2 \\
\hline \multirow[t]{2}{*}{ PE (\%) } & 100.6 & 67.2 & 104.5 & 86.0 & 86.5 & 110.6 & 94.9 \\
\hline & \multicolumn{7}{|c|}{ Medium/High (25 ng/mL) } \\
\hline ME (\%) & 95.6 & 70.2 & 98.1 & 97.1 & 100.8 & 142.7 & 98.3 \\
\hline RE (\%) & 94.0 & 225.4 & 94.4 & 94.3 & 99.6 & 94.3 & 94.0 \\
\hline \multirow[t]{2}{*}{ PE (\%) } & 89.9 & 158.2 & 92.7 & 91.6 & 100.4 & 134.7 & 92.4 \\
\hline & \multicolumn{7}{|c|}{ Medium/Low (5 ng/mL) } \\
\hline ME (\%) & $\overline{94.9}$ & 14.7 & 106.2 & 100.8 & 91.7 & 256.8 & $\overline{102.4}$ \\
\hline RE (\%) & 99.5 & 73.8 & 101.8 & 98.9 & 105.4 & 109.2 & 100.2 \\
\hline PE (\%) & 94.4 & 10.9 & 108.1 & 99.7 & 96.7 & 290.4 & 102.6 \\
\hline & \multicolumn{7}{|c|}{ Low $(0.5 \mathrm{ng} / \mathrm{mL})$} \\
\hline ME (\%) & 79.5 & - & 88.0 & 81.0 & - & 144.7 & 85.3 \\
\hline RE (\%) & 92.8 & - & 95.0 & 94.8 & - & 100.3 & 92.8 \\
\hline PE (\%) & 73.8 & - & 83.6 & 76.7 & - & 145.1 & 79.2 \\
\hline
\end{tabular}


Table 46 - Matrix Effects, Recovery, and Process Efficiency for Fentanyl and Metabolites in RCM

\begin{tabular}{|c|c|c|c|}
\hline Analyte & FENT & NFENT & DESP \\
\hline & \multicolumn{3}{|c|}{ High $(200 \mathrm{ng} / \mathrm{mL})$} \\
\hline ME (\%) & 88.0 & 93.9 & 93.6 \\
\hline RE (\%) & 98.8 & 99.7 & 98.7 \\
\hline \multirow[t]{2}{*}{ PE (\%) } & 86.9 & 93.7 & 92.4 \\
\hline & \multicolumn{3}{|c|}{ Medium/High $(25 \mathrm{ng} / \mathrm{mL})$} \\
\hline ME (\%) & 96.9 & 97.7 & 97.7 \\
\hline RE (\%) & 94.9 & 94.5 & 96.0 \\
\hline \multirow[t]{2}{*}{ PE (\%) } & 92.0 & 92.3 & 93.8 \\
\hline & \multicolumn{3}{|c|}{ Medium/Low (5 $\mathrm{ng} / \mathrm{mL})$} \\
\hline ME (\%) & 102.9 & 103.1 & 106.0 \\
\hline RE (\%) & 99.9 & 100.6 & 98.3 \\
\hline PE (\%) & 102.8 & 103.7 & 104.3 \\
\hline & \multicolumn{3}{|c|}{ Low $(0.5 \mathrm{ng} / \mathrm{mL})$} \\
\hline ME (\%) & 88.4 & 81.0 & 85.8 \\
\hline RE (\%) & 95.6 & 95.0 & 94.8 \\
\hline PE (\%) & 84.5 & 76.9 & 81.3 \\
\hline
\end{tabular}

The ME, RE, and PE reflect the ability and efficiency of the SPE method. Matrix effects showed slight ionization enhancement for $\mathrm{COC}$, BZE, and $\mathrm{mHC}$ at high concentration. The other analytes experienced ionization suppression with ME ranging from $87.5 \%$ to $96.7 \%$. RCM is a complex medium and both ionization enhancement as well as suppression effects can be contributed to matrix components.

AEME experienced matrix effects of $49.4 \%$ even at high concentration. This apparent suppression was a result of several factors. There was no deuterated AEME standard in this method, and using EME-d3 may not have been adequate for accurate quantitation. Additionally, AEME was a very polar molecule and eluted near the solvent front. This could cause ionization to be incomplete as well as inconsistent. Finally, 
AEME eluted soon after EME and their MRM transition peaks exhibited some tailing. These two molecules differed only by a water molecule and the most abundant transition of EME was the $182.0 \mathrm{~m} / \mathrm{z}$ of AEME. This transition was not selected to be monitored for EME, but with the analytes eluting so close together, the RT window could not be narrowed enough to isolated the analyses. All of these factors could explain the method effects on AEME individually, but most likely a combination of effects was responsible. The RE and PE of AEME were also affected across all concentration levels. Analysis of AEME will be very limited in this method.

ME were more prominent for the other analytes at low concentration with suppression ranging from 79.5 to $85.8 \%$. $\mathrm{mHC}$ is the only analyte at low concentrations to express ionization enhancement at $144.7 \%$. This could be attributed to the lack of deuterated $\mathrm{mHC}$. BZE may not have accurately defined the responses of $\mathrm{mHC}$ at lower concentrations which resulted in increased ionization enhancement. It could also be attributed to matrix components that interfered with $\mathrm{mHC}$ ionization and signal response.

Recovery ranged from 92.8 to $109.2 \%$ across all concentration levels for both sets of analytes and metabolites (except AEME). This indicated that the SPE method was efficient in retrieving analytes from the matrix. However, the method was not as successful in removing matrix components as evidenced by the extent of ME on all analytes. Perhaps an additional wash step or maybe a syringe filtering could be applied to improve the clean up. The RE \% was not calculated for EME at $0.5 \mathrm{ng} / \mathrm{mL}$ because it was below the LOQ. 
PE was designed to take ionization effects as well as recovery into account. PE ranged from 86.0 to $110.6 \%$ for high and medium concentration levels for COC, $\mathrm{BE}, \mathrm{CE}$, NC, FENT, NFENT, and DESP, and 73.8 to $84.5 \%$ at low concentration. PE ranged from 110.6 to $290.4 \%$ for $\mathrm{mHC}$ while recoveries only ranged from $94.3-109.2 \%$ across all concentrations. The high PE values are therefore due to matrix effects. Over all, the FENT and metabolites were much less affected by ME than COC and metabolites. FENT analytes also had significantly better recoveries across all concentration levels.

\subsubsection{Bias and Precision}

Bias and precision (within-run precision) and repeatability (between-run precision) were defined in Chapter 1. The values were calculated using Equation 4, Equation 5, and Equation 6, respectively. Analysis of percent bias and within-run and between-run precision was conducted at four different concentrations 100 (high), 10 (medium/high), 5 (medium/low), and $0.5 \mathrm{ng} / \mathrm{mL}$ (low) for COC, BZE, CE, $\mathrm{mHC}, \mathrm{NC}$, FENT, NFENT, and DESP. EME studies were conducted only at the high, medium/high, and medium/low concentrations, and AEME only at the high and medium/high concentrations due to LOD and LOQ limitations. Data is summarized in Table 47 and Table 48. 
Table 47 - Bias, Within-Run and Between-Run \%CV for Cocaine and Metabolites in $\mathrm{RCM}$

\begin{tabular}{|c|c|c|c|c|c|c|c|}
\hline Analyte & COC & AEME & BZE & CE & EME & $\mathrm{mHC}$ & NC \\
\hline & \multicolumn{7}{|c|}{ High (100 ng/mL) } \\
\hline Bias (\%) & -7.7 & -9.6 & -7.4 & -8.3 & -11.0 & -13.2 & -9.9 \\
\hline Within-Run (\%) & 2.2 & 7.1 & 2.1 & 1.7 & 1.2 & 3.2 & 2.5 \\
\hline \multirow[t]{2}{*}{ Between-Run (\%) } & 6.8 & 7.3 & 4.5 & 3.5 & 3.8 & 4.3 & 5.8 \\
\hline & \multicolumn{7}{|c|}{ Medium/High (10 ng/mL) } \\
\hline Bias (\%) & -8.2 & 3.1 & -8.6 & -5.7 & -5.8 & -11.4 & $\overline{10.3}$ \\
\hline Within-Run (\%) & 2.0 & 3.4 & 2.7 & 1.8 & 1.6 & 2.2 & 1.6 \\
\hline \multirow[t]{2}{*}{ Between-Run (\%) } & 10.1 & 12.2 & 10.9 & 9.8 & 12.8 & 11.3 & 9.9 \\
\hline & \multicolumn{7}{|c|}{ Medium/Low (5 ng/mL) } \\
\hline Bias (\%) & -5.2 & - & -3.6 & -12.7 & -16.0 & -19.6 & -9.0 \\
\hline Within-Run (\%) & 1.7 & - & 3.0 & 1.8 & 1.0 & 2.2 & 6.0 \\
\hline \multirow[t]{2}{*}{ Between-Run (\%) } & 7.9 & - & 4.5 & 8.1 & 4.1 & 3.9 & 11.0 \\
\hline & \multicolumn{7}{|c|}{ Low (0.5 ng/mL) } \\
\hline Bias (\%) & $\overline{-17.4}$ & - & -5.0 & -18.4 & - & -15.8 & $\overline{-11.1}$ \\
\hline Within-Run (\%) & 4.4 & - & 7.2 & 7.8 & - & 7.2 & 7.0 \\
\hline Between-Run (\%) & 4.8 & - & 6.5 & 5.3 & - & 7.0 & 5.4 \\
\hline
\end{tabular}


Table 48 - Bias, Within-Run and Between-Run \%CV for Fentanyl and Metabolites in RCM

\begin{tabular}{|c|c|c|c|}
\hline Analyte & FENT & NFENT & DESP \\
\hline & \multicolumn{3}{|c|}{ High (100 ng/mL) } \\
\hline Bias (\%) & -11.6 & -6.5 & -11.5 \\
\hline Within-Run (\%) & 1.1 & 1.4 & 2.1 \\
\hline \multirow[t]{2}{*}{ Between-Run (\%) } & 3.3 & 4.3 & 3.6 \\
\hline & \multicolumn{3}{|c|}{ Medium/High $(10 \mathrm{ng} / \mathrm{mL})$} \\
\hline Bias (\%) & -10.4 & -7.1 & -9.7 \\
\hline Within-Run (\%) & 2.2 & 1.5 & 1.1 \\
\hline \multirow{2}{*}{ Between-Run (\%) } & 11.9 & 9.5 & 13.8 \\
\hline & \multicolumn{3}{|c|}{ Medium/Low (5 $\mathrm{ng} / \mathrm{mL})$} \\
\hline Bias (\%) & -19.0 & -5.8 & -15.2 \\
\hline Within-Run (\%) & 2.5 & 1.9 & 1.1 \\
\hline \multirow[t]{2}{*}{ Between-Run (\%) } & 8.1 & 8.2 & 7.2 \\
\hline & \multicolumn{3}{|c|}{ Low (0.5 ng/mL) } \\
\hline Bias (\%) & -11.3 & -18.8 & -15.0 \\
\hline Within-Run (\%) & 6.1 & 5.3 & 5.8 \\
\hline Between-Run (\%) & 7.0 & 7.2 & 6.7 \\
\hline
\end{tabular}

Within-run precision ranged from 1.1 to $7.8 \%$ across each of the concentration levels while between-run precision ranged from 3.3 to $13.8 \%$. For most analytes the higher concentration sample sets demonstrated the narrowest range of values.

Generally, bias and precision values increased as concentration values decreased toward the LOD and LOQ values of the analytes. Even though the acceptable range is $\pm 20 \%$ is the same for all concentration levels, small changes in sample extraction and preparation as well as instrumental response have a greater effect on lower concentrations than higher concentrations. This trend was also seen with DZ, FLU, and their metabolites. 


\subsubsection{Dilution Integrity}

Dilution integrity was determined by repeating bias and precision studies on diluted samples. Samples were prepared at $1000 \mathrm{ng} / \mathrm{mL}$ and then diluted to 1:5 (200 $\mathrm{ng} / \mathrm{mL})$ and 1:10 $(100 \mathrm{ng} / \mathrm{mL})$. Five samples were prepared and analyzed in triplicate for each concentration. Both dilution sets maintained requisite identification criteria for all analytes except for AEME. Excluding AEME, the bias ranged from -10.6 to $14.0 \%$, within-run precision from 0.7 to $2.0 \%$, and between-run precision from 1.7 to $7.4 \%$. The bias for AEME in the 1:10 dilution set was higher than $\pm 20 \%$. Future dilutions should be prepared at 1:5. The summarized data can be seen in Table 49 and Table 50.

Table 49 - Dilution Integrity for Cocaine and Metabolites in RCM

\begin{tabular}{|cccccccc|}
\hline Analyte & COC & AEME & BZE & CE & EME & mHC & NC \\
\hline \multicolumn{7}{c|}{$\mathbf{1 : 5}(\mathbf{1 0 0 0}: \mathbf{2 0 0} \mathbf{~ n g / m L ) ~}$} \\
\hline Bias (\%) & -3.5 & -19.8 & -5.9 & 5.5 & 2.7 & 14.0 & 1.2 \\
\hline Within-Run (\%) & 0.7 & 1.2 & 1.4 & 1.3 & 1.2 & 1.5 & 1.3 \\
\hline Between-Run (\%) & 4.3 & 7.4 & 4.7 & 4.7 & 4.4 & 5.4 & 4.6 \\
\hline \multicolumn{7}{c}{$\mathbf{1 : 1 0}(\mathbf{1 0 0 0}: 100$} & $\mathbf{n g} / \mathbf{m L})$ \\
\hline Bias (\%) & -4.9 & -25.3 & -9.0 & 4.1 & -1.0 & 9.8 & -1.0 \\
Within-Run (\%) & 1.5 & 1.3 & 1.4 & 1.3 & 2.0 & 1.6 & 1.2 \\
Between-Run (\%) & 2.6 & 3.4 & 2.6 & 2.7 & 2.7 & 1.7 & 2.4 \\
\hline
\end{tabular}


Table 50 - Dilution Integrity for Cocaine, Fentanyl, and in RCM

\begin{tabular}{|c|c|c|c|}
\hline Analyte & FENT & NFENT & DESP \\
\hline & \multicolumn{3}{|c|}{ 1:5 (1000:200 ng/mL) } \\
\hline Bias (\%) & -1.0 & 9.0 & -7.5 \\
\hline Within-Run (\%) & 1.6 & 1.5 & 1.2 \\
\hline Between-Run (\%) & 4.4 & 5.2 & 4.7 \\
\hline & \multicolumn{3}{|c|}{ 1:10 (1000:100 ng/mL) } \\
\hline Bias (\%) & -4.1 & 5.4 & -10.6 \\
\hline Within-Run (\%) & 1.5 & 1.5 & 1.1 \\
\hline Between-Run (\%) & 2.8 & 2.4 & 2.7 \\
\hline
\end{tabular}

\subsubsection{LOD and LOQ}

LOD and LOQ were defined in Sections 1.5 .7 and 1.5.8, respectively. Values for COC, FENT, and metabolites ranged from 0.100 to $10 \mathrm{ng} / \mathrm{mL}$ and are reported in Table 2. $C O C$ and $B Z E$ achieved the lowest $L O D$ and $L O Q$ values at $0.1 \mathrm{ng} / \mathrm{mL}$. $\mathrm{mHC}, \mathrm{NC}$, FENT, and DESP maintained RT, peak shape, and ion ratios at $0.1 \mathrm{ng} / \mathrm{mL}$, satisfying LOD requirements but bias exceeded $\pm 20 \%$. The LOQ requirements were fulfilled at $0.25 \mathrm{ng} / \mathrm{mL}$ for these analytes. All of the analytes of interest had LOQ values $\leq 0.5$ $\mathrm{ng} / \mathrm{mL}$ with the exceptions of AEME and EME. Both of these analytes eluted near or possibly with the solvent front. This caused ionization to be incomplete as well as inconsistent, which resulted in higher LOD and LOQ values. The lack of a deuterated AEME standard also affected quantitation at lower concentrations.

\subsubsection{Stability}

Stability was assessed by evaluating freeze-thaw stability as well as processed sample stability., which were defined in Section 1.5.11. Freeze-thaw stability was 
conducted over three cycles at three concentrations $200 \mathrm{ng} / \mathrm{mL}$ (high), $10 \mathrm{ng} / \mathrm{mL}$ (medium), and $0.5 \mathrm{ng} / \mathrm{mL}$ (low).

COC, BZE, CE, NC, and NFENT remained stable for all three concentrations and FT cycles. EME was stable for all three cycles at the high and medium concentrations, however the low concentration sample was below the LOQ of EME. Fentanyl was stable for FT1, FT2, and FT3 for high concentration, and FT1 and FT2 for medium concentration. All concentrations were stable for FT1 and FT2 for mHC and DESP. With the exception of AEME, most analytes should remain stable through two complete freeze-thaw cycles at high and medium concentrations. Experimental values for AEME were well above the theoretical values for all concentrations and cycles. Future analyses involving quantitation of AEME should therefore be conducted immediately after preparation and not preserved by freezing.

The stability of processed samples was studied at two concentrations $200 \mathrm{ng} / \mathrm{mL}$ (high) and $5 \mathrm{ng} / \mathrm{mL}$ (low) in triplicate over the course of 60 hours. Samples were taken every 12 hours to accommodate the sample run time. For $200 \mathrm{ng} / \mathrm{mL}$, cocaine and metabolites remained stable until the 48 hour mark. At 60 hours, however, $\mathrm{mHC}$ dropped below the $160-240( \pm 20 \%) \mathrm{ng} / \mathrm{mL}$ range (dashed lines). Error bars were determined at the $95 \%$ confidence interval. This was depicted in Figure 33. Fentanyl and metabolites remained stable for the entirety of the 60 hour stability testing as seen in Figure 34. 
Figure 33 - Processed Sample Stability for Cocaine and Metabolites in RCM at High Concentration (200 ng/mL)

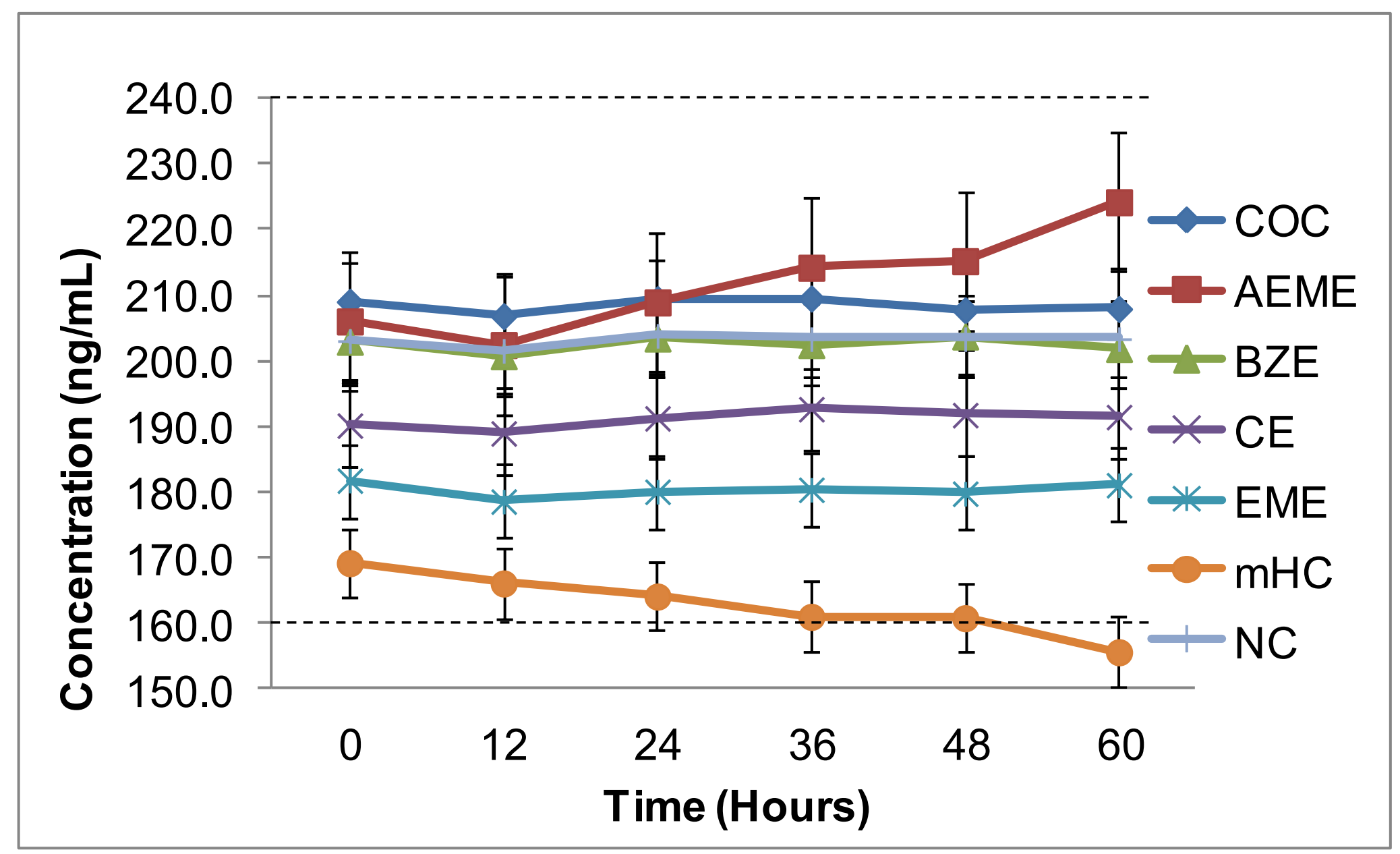


Figure 34 - Processed Sample Stability for Fentanyl and Metabolites in RCM at High Concentration (200 ng/mL)

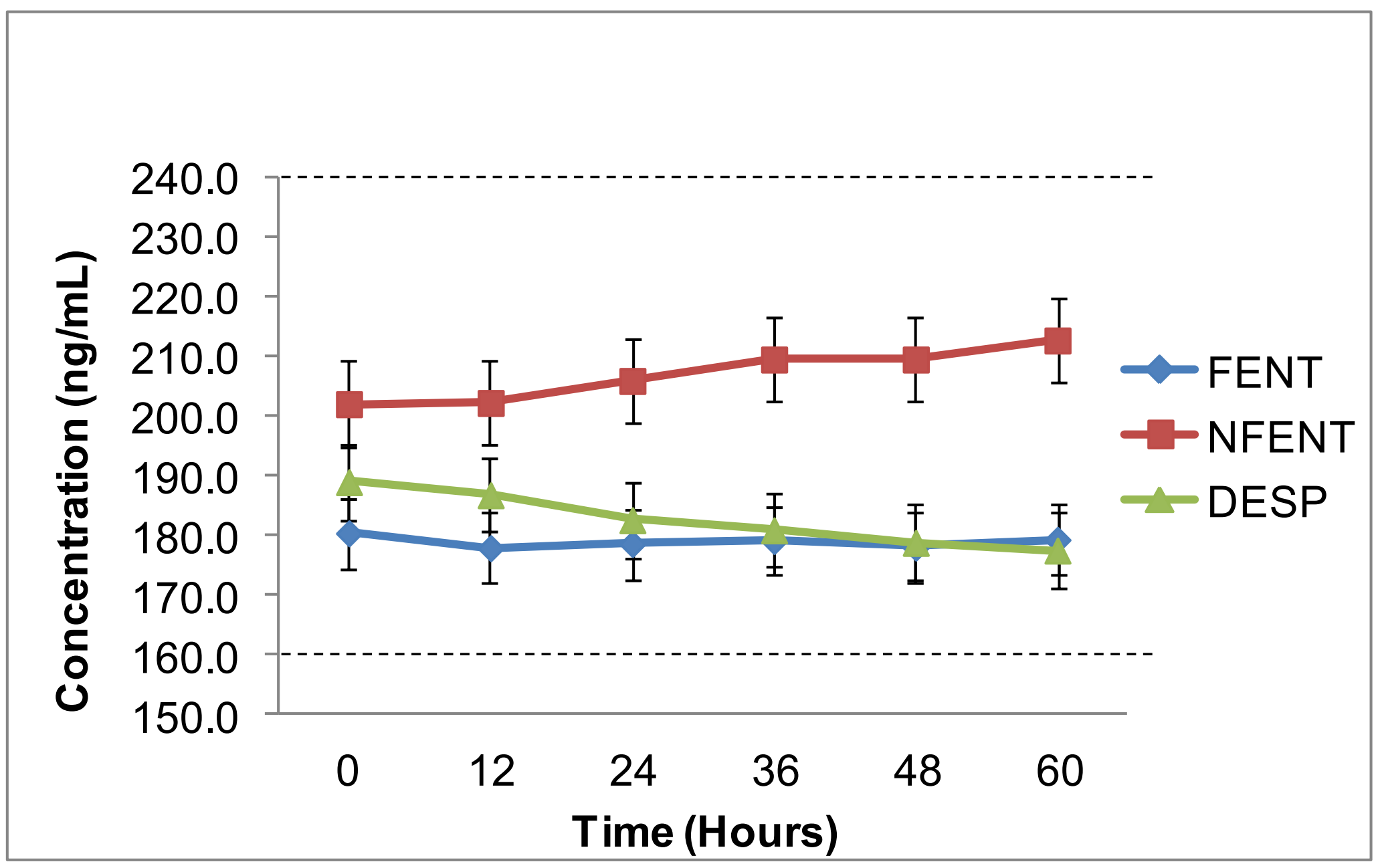


At low concentration ( $5 \mathrm{ng} / \mathrm{mL}), \mathrm{COC}$ and metabolites remained stable for at least 60 hours (Figure 35) as did FENT and metabolites (Figure 36). Error bars were determined at the $95 \%$ confidence interval, and dashed lines represent the $\pm 20 \%$ range. The stability of AEME was not assessed at the low concentration because it was below the LOQ for that particular metabolite. Based on these studies, samples should remain stable for at least 48 hours. This was an important feature of method validation, especially in cases of large sample batches as well as periods of instrumental malfunction. 
Figure 35 - Processed Sample Stability for Cocaine and Metabolites in RCM at Low Concentration ( $5 \mathrm{ng} / \mathrm{mL})$

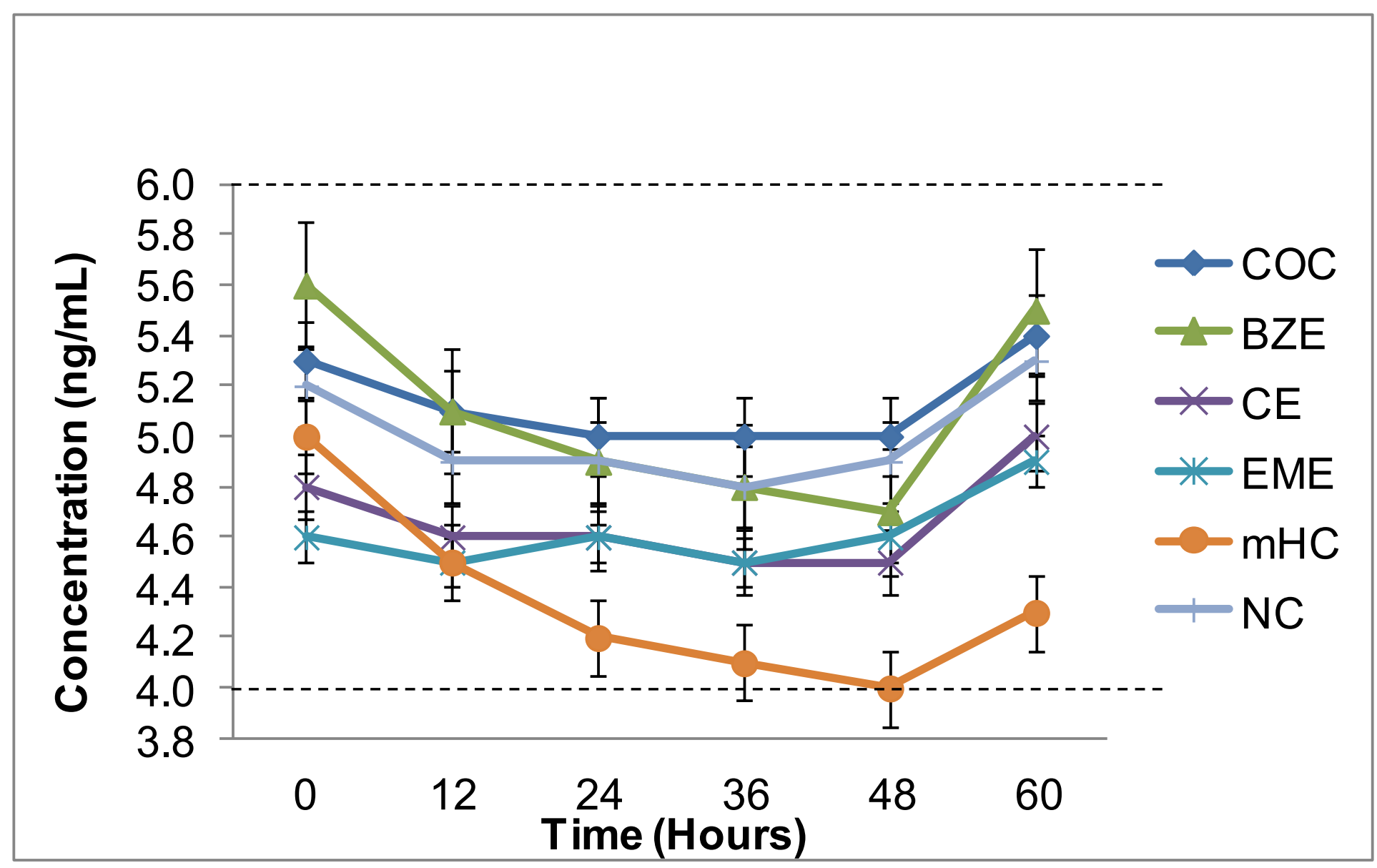


Figure 36 - Processed Sample Stability for Fentanyl and Metabolites in RCM at Low Concentration $(5 \mathrm{ng} / \mathrm{mL})$

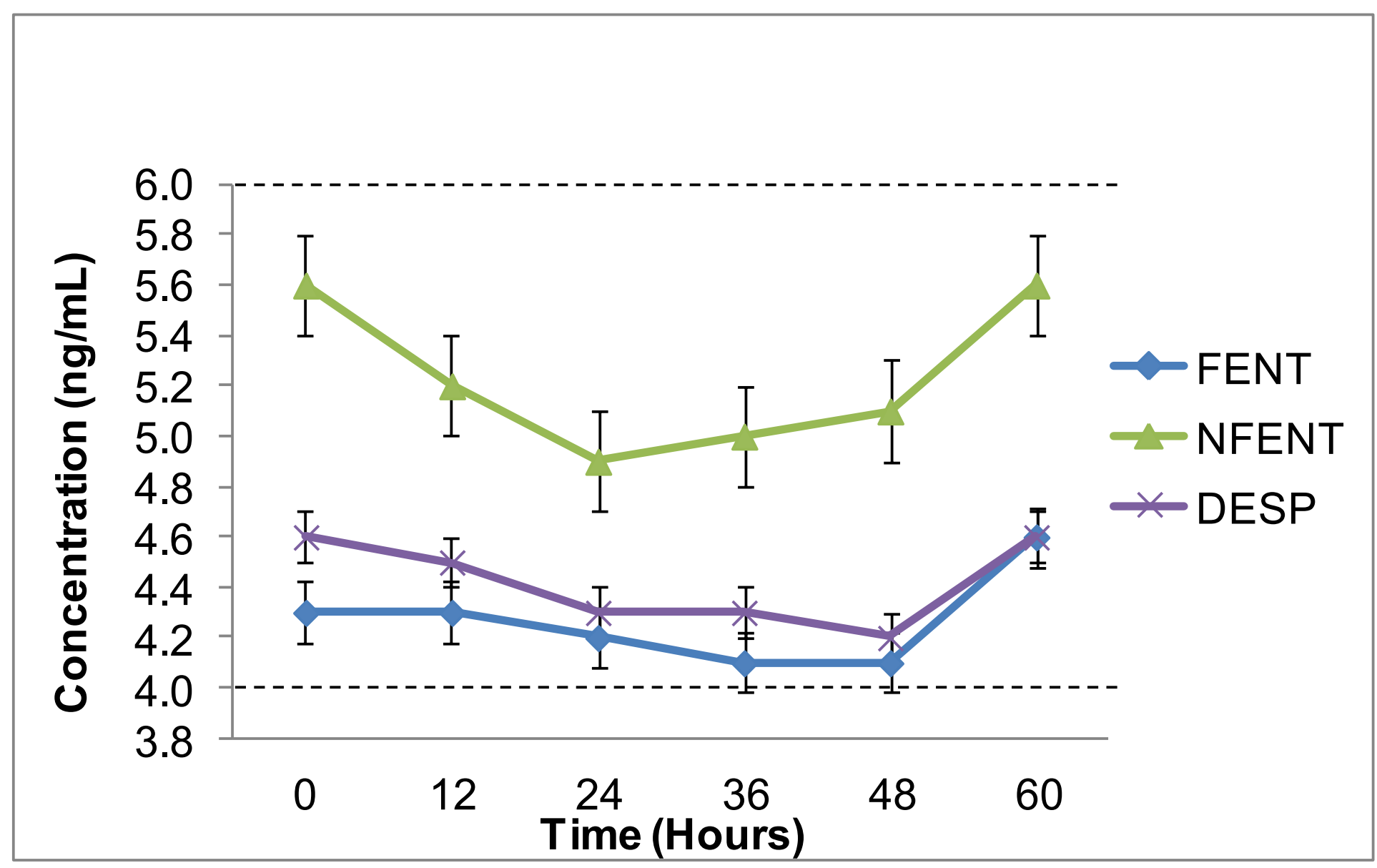




\subsubsection{Conclusions}

An LC-MS/MS method was developed and validated for the detection, identification, and quantitation of COC, FENT, and metabolites extracted from RCM. This method can be used to apply the analyte degradation and biotransformation studies outlined in Chapter 4 for DZ and FLU to COC, FENT, and other drugs of abuse and forensic importance. 


\section{Chapter 6: Research Summary, Implications, and Future Works}

\subsubsection{Summary of Research}

A review of previously published literature and research revealed that bacterial species were capable of metabolizing complex chemical substances. Many of the biotransformation applications discussed in Chapter 1 resulted in novel and interesting applications toward agricultural and environmental contaminant reduction or elimination, greater insight into the effectiveness of pharmaceutical compounds, and new applications of isolated bacterial enzymes.

Some of these same bacterial species are native to the human GI tract and play an active role in the postmortem decomposition process. These species have potential to cause biotransformations that affect the ratios of compound-to-metabolite concentrations within the human body. Such effects have rarely been considered or evaluated, but they have potential to supply valuable information, especially concerning compound identification and confirmation.

The purpose of this research was to develop methodology, investigate the effects of $\mathrm{Gl}$ bacteria on drugs of abuse, and to compare metabolites to those recovered in human metabolic studies. Analytes of interest and (metabolites) were DZ (NDZ, OX, TZ), FLU (7AM, NDES), COC (AEME, BZE, CE, EME, mHC, NC), and FENT (NFENT, DESP). All of these compounds are scheduled drugs of abuse controlled by the DEA, and for which there have been numerous studies revolving around human metabolism. 
The analytes and selected metabolites were also chosen based on the commercial availability of certified reference standards.

Bacterial growth and preparation techniques were optimized and discussed in Chapter 2, which included nutrient medium evaluation and selection, establishment and maintenance of anaerobic conditions, preparation of frozen stock cultures, viable bacterial enumeration, and a review of cellular lysis techniques. E. coli, B. fragilis, and C. perfringens were selected because they are prominent species, viable under the limited oxygen conditions found in the human GI tract and thereby capable of growth and metabolism. RCM was chosen as the nutrient medium because it was able to support the growth and proliferation of all three bacterial species. Anaerobic conditions were established using an anaerobic chamber fitted with purge gases that worked with a catalyst system to convert residual oxygen to $\mathrm{H}_{2} \mathrm{O}$. Maintenance of these conditions was critical in supporting bacterial growth, particularly for B. fragilis and C. perfringens, anaerobic species whose growth and survival were inhibited in the presence of oxygen.

Preparation of a frozen stock culture for each species provided an ample supply of bacteria for biotransformation studies, and the plate count procedure gave an estimation of the viable bacteria in inoculated samples. The $\mathrm{CFU} / \mathrm{mL}$ counts were within the ranges of viable populations found in the human GI tract, specifically the ileum $\left(10^{5}-10^{8}\right)$ and colon $\left(10^{10}-10^{12}\right)$ as reported by the $\mathrm{NIH}{ }^{45}$ Several cellular lysis methods were evaluated, but all were dismissed to maintain the accuracy and integrity of the optimized experimental conditions.

To investigate the effects of the selected bacterial species on DZ and FLU, an LC-MS/MS method was developed for the detection, identification, and quantitation of 
these benzodiazepine compounds as well as their respective metabolites. The method was fully validated to ensure analysis was performed and interpreted with reliability and certainty. Analytes were recovered from RCM via SPE. To date, similar methods of analysis and quantitation of drugs of abuse in a nutrient medium matrix have not been published. Method development and validation were discussed in Chapter 3.

Validation was based on definitions and parameters established by the $\mathrm{ICH}^{25}, 89$ SWGTOX ${ }^{26}$, and DHHS, FDA, CDER, and CVM. ${ }^{27}$ Linearity was addressed by creating a calibration model for each of the analytes in RCM. Specificity was evaluated through matrix and internal standard interference studies, ionization enhancement/suppression, ME, RE, and PE. The carryover effects, accuracy, bias, precision (within-run precision), repeatability (between-run precision), LOD, LOQ, range, dilution integrity, freeze-thaw stability, processed sample stability, and robustness of the methods were also assessed.

Dilution integrity was particularly important because biotransformation samples were spiked at $1000 \mathrm{ng} / \mathrm{mL}$, while the upper range of the method was only $500 \mathrm{ng} / \mathrm{mL}$. Dilutions were prepared at 1:2 and 1:5. Both sets met the requirements of maintaining acceptable retention time, peak shape, an ion ratios as well as accuracy, bias, and within-run and between-run precision. Processed sample stability was also important. Analytes were incubated for 48 hours as part of the experimental application process. In addition, samples were analyzed in large batches and with replicate analysis which prolonged the time between introduction to the autosampler and LC-MS/MS analysis. The processed sample stability study provided that samples would maintain stability for at least 48 hours after extraction from RCM. 
Following validation, the method was applied to assess the specific effects of $E$. coli, B. fragilis, and C. perfringens on DZ and FLU, which were discussed in Chapter 4. Experiments explored the effects of environmental conditions on analyte blanks as well as bacterial inoculates. Biotransformation studies explored the effects of individual species as well as a mixed culture on the benzodiazepines compounds under anaerobic conditions.

A series of bacterial blanks were prepared in RCM with aliquots of E. coli, B. fragilis, C. perfringens, or a mixed culture without analytes of interest. These samples were incubated under experimental conditions. None of the bacterial specimens produced interferences with the target analytes that satisfied the requirements for peak shape, retention time, ion ratios, and $\mathrm{LOQ}$.

Analyte blanks were also examined. RCM spiked with either DZ or FLU was incubated and analyzed. Mass balance data for DZ suggested it undergoes a partial, unassisted degradation when incubated under experimental conditions. However, NDZ, OX, nor TZ registered an increase from pre- to post-incubation concentrations. The proposed degradation product was therefore unidentified.

RCM samples prepared for biotransformation studies were spiked with an analyte of interest at $1000 \mathrm{ng} / \mathrm{mL}$ as well as either E. coli, B. fragilis, C. perfringens, or mixed culture. Pre- and post-incubation aliquots were taken for analysis and the change in concentration results were calculated.

Analysis of the effects of $E$. coli on DZ concentration produced an average DZ concentration difference that was slightly greater than what was observed in DZ blank samples. Consistent with the drug blank samples, NDZ, OX, nor TZ registered a 
increase from pre- to post-incubation concentrations. The mass balance discrepancies between blank and biotransformation study samples indicated that there may be additional factors contributing to the transformation of $D Z$ when incubated with E.coli. Similar results were seen in the $B$. fragilis biotransformation studies as well.

In both cases RCM samples showed the marked turbidity indicative of prominent bacterial growth. A possible explanation for the discrepancy between DZ blank and $E$. coli and $B$. fragilis biotransformation samples is that $D Z$ was being converted to a compound other than the monitored human metabolites. In this case, E. coli and B. fragilis would have possessed a specific enzyme or combination of enzymes capable of cleaving DZ to form alternative metabolites. It is unclear whether E.coli and B. fragilis would have employed the same enzyme or if the DZ conversion was attributed to species-specific enzymes. Additional experimental and literature review may offer some guidance toward alternative metabolites.

Similar to the studies performed on $E$. coli and $B$. fragilis, there were negligible changes in concentration detected for NDZ, OX, and TZ in samples inoculated with $C$. perfringens. However, there was minimal change in DZ concentration as well. These findings supported the theory of $E$. coli and $B$. fragilis were responsible for the concentration discrepancies between the DZ drug blanks and their respective biotransformation studies. The small change in DZ concentration, however, was unique to $C$. perfringens incubations. All samples were prepared and incubated under the same circumstances and a loss of DZ concentration comparable to at least that of the drug blank samples was anticipated. Since DZ experienced minimal concentration loss, 
it is possible that introduction of $C$. perfringens stabilized the analyte and prevented degradation.

The effects of the mixed culture studies resulted in post-incubation concentrations similar to those seen in C. perfringens. This supported the supposition that the presence of $C$. perfringens stabilized the analyte and prevented the proposed degradation by $E$. coli and B. fragilis.

Analysis of the FLU drug blanks exhibited a decrease in concentration. However, unlike what was observed in the DZ blank experiments, there was a corresponding increase in concentration for the 7AM metabolite in the post-incubation samples that accounted for some of the FLU loss. E. coli, B. fragilis, C. perfringens, and the mixed culture were all capable of transforming FLU to 7AM to some extent. Data was consistent with that published in experiments by Robert and Drummer ${ }^{16,17}$ in which they studied effects of GI bacteria on three benzodiazepine compounds in blood. They discovered that all three compounds were converted to their respective 7-amino metabolites though the rate of conversion varied amongst the different species.

E. coli was capable of converting a portion of the FLU to 7AM between pre- and post-incubation samples, which accounted for some of the FLU loss. However, not all of the original FLU concentration was recovered as 7AM as seen in the mass balance of the conversion. This could potentially be attributed to FLU being transformed into an additional metabolite that was not monitored such as 3-hydroxyflunitrazepam. Another possibility was that 7AM itself was being converted to metabolites 7-acetaminoflunitrazepam and/or 7-amino-nor-flunitrazepam. Structures for these three structures 
were presented in Figures 23 and 24. Further analysis would be required to determine the presence and identity of these or other potential bacterial metabolites.

B. fragilis, C. perfringens, and the mixed culture completely transformed the preincubation FLU concentration in all samples. The majority was recovered as 7AM in the post-incubation samples, but there was some concentration discrepancy. The metabolites proposed above were the most likely candidates for minor metabolites, but further analysis would be required.

An additional drugs of abuse and (metabolites) method was developed and fully validated for COC (AEME, BZE, CE, EME, $\mathrm{mHC}$, and NC) and FENT (NENT and DESP) in RCM, the results of which were discussed in Chapter 5. It was validated in similar fashion to the method for DZ and FLU. As with the DZ and FLU method, dilution integrity was particularly important. Dilution integrity dictated that samples would be diluted 1:5 prior to analysis. Processed sample stability was likewise an important feature of the method validation. The processed sample stability study provided that samples would maintain stability for 48 hours after extraction from RCM. This method is awaiting application to study the effects of E. coli, B. fragilis, and C. perfringens on COC and FENT.

\subsubsection{Research Implications}

The experimental results served as a pilot study and proof of concept. Though the ability of bacterial species to transform complex chemical substances has been previously studied, this methodology offers several distinct advantages. Much of the previous research relied on germfree, gnobiotic, and conventional rats or excretions 
from volunteer human subjects. The presented methodology utilized bacterial species for experimental purposes which eliminated the ethical considerations and guidelines required for animal and human research endeavors.

The methodology was also cost-effective. Preparation of frozen stock cultures for each bacterial species provided a theoretically endless supply of specimens for transformation studies, which aided in keeping the materials costs to a minimum. Extraction procedures, a narrow bore column, and low flow rate for instrumental analysis minimized solvent consumption and hazardous waste production, which was both cost-effective and environmentally friendly. In addition, the streamlined experimental approach allowed for quantities of experimental variations to be performed in unison, which conserved time.

The presented experimental methodology serves as a template for further exploration of the effects of bacterial species on complex chemical compounds. The principles behind experimental design and analysis can be adapted and applied to a realm of possibilities. But perhaps the most important feature is the ability to streamline experimental procedures.

These methods could be applied concurrently to determine bacterial species candidates for biodegradation of agricultural and environmental contaminants, such as pesticides, explosives, and chemical warfare agents. They can also be utilized to isolate and repurpose native bacterial enzymes. This has seen promise in the medical field as well as in sensors for drugs of abuse detection. The methods can likewise be used in further studies concerning bacterial effects on pharmaceutical compounds and 
drugs of abuse. In addition to expanding the analytes of interest, the applications can easily be expanded to include alternative bacterial species as well.

Ultimately, this methodology would be ideal to study compounds that are too toxic or lethal for animal and human metabolic investigations. This would be particularly useful in military explorations of exposure to incapacitation and chemical warfare agents. In many of these instances, victims do not survive long enough for the compound to undergo human metabolic conversions. Since bacterial species are capable of transmigration postmortem as well as biotransformation of complex chemical compounds, their metabolites could provide information that would be useful in agent or compound identification.

\subsubsection{Future Work}

COC and FENT are of forensic importance. Future works should begin with the application of the validated method for COC and FENT discussed in Chapter 5. These analytes can easily be incorporated into analyte blank and biotransformation studies described in Chapter 4. Development of the presented methodology and analysis of results presented several other experimental opportunities that could expand upon and contribute to this area of research.

Kinetic studies could be performed to determine the degradation rate of DZ and FLU in the analyte blank samples discussed in Chapter 4. Proposed experimental design could be based upon processed sample stability studies performed as a part of method validation (Section 3.3.8), where an aliquot of analyte blank is taken at defined time intervals over the course of the 48 hour incubation period, extracted, and analyzed 
via LC-MS/MS. The change in analyte concentration over time would provide an estimate of the unassisted degradation of DZ and FLU under experimental conditions. This conversion rate could also be compared to that calculated for each analyte when incubated with E. coli, B. fragilis, C. perfringens, and mixed culture in biotransformation studies. Kinetic studies could be conducted for COC and FENT conversions as well. Identification of the other degradation and biotransformation products would likewise be an important contribution to the DZ and FLU experiments.

Robertson and Drummer studied the effects of $\mathrm{pH}$ and temperature on the compound-to-metabolite rate of conversion, which were discussed in Section 1.1.3. Changes in the environmental and incubation conditions could be applied to study the conversion rate of FLU to 7AM using the methods provided in Chapters 3 and 4 as well as the proposed kinetic studies. ${ }^{16,17}$

The presented experimental methodology provides a template for further exploration of the effects of bacterial species on complex chemical compounds. These techniques can be expanded to include other drugs of abuse. Preliminary methods have been developed for methadone (Appendix F) and methamphetamine (Appendix G). These methods utilize the same HPLC buffer system and could be combined for convenience, validated, and applied to the biotransformation studies. Other compounds of interest such as pharmaceuticals, environmental contaminants, and explosives could also be incorporated for analysis.

Table 4 lists the prevalent bacteria in the lower GI tract, all of which could be incorporated into the methodology. Experiments could also be expanded to include bacterial species other than those in the human GI tract. This methodology focused on 
facultative anaerobic and anaerobic species. However, experiments could be expanded to include aerobic species as well. E.coli could be used to compare the effects of aerobic vs. anaerobic conditions on biotransformation products and rates.

This method was applied to biotransformation studies of the benzodiazepine analytes by GI bacteria. This method and application served as a proof of concept, proving that this type of experimentation and methodology are capable of studying bacterial metabolism of complex compounds. The methodology can expand to encompass other drugs of abuse or compounds on interest as well as incorporate other bacterial species. 


\section{References}

1. Peppercorn, M.; Goldman, P., Caffeic Acid Metabolism by Bacteria of Human Gastrointestinal Tract. Journal of Bacteriology 1971, 108 (3), 996-1000.

2. Peppercorn, M.; Goldman, P., Caffeic Acid Metabolism by Gnobiotic Rats and Their Intestinal Bacteria. Proceedings of the National Academy of Sciences of the United States of America 1972, 69 (6), 1413-1415.

3. Islam, R.; Zhou, T.; Young, J. C.; Goodwin, P. H.; Pauls, K. P., Aerobic and Anaerobic De-epoxydation of Mycotoxin Deoxynivalenol by Bacteria Originating from Agricultural Soil. World Journal of Microbiology \& Biotechnology 2012, 28 (1), 7-13.

4. Bharagava, R. N.; Chandra, R., Biodegradation of the Major Color Containing Compounds in Distillery Wastewater by an Aerobic Bacterial Culture and Characterization of Their Metabolites. Biodegradation 2010, 21 (5), 703-711.

5. Mohamed, M. S., Degradation of Methomyl by the Novel Bacterial Strain Stenotrophomonas maltophilia M1. Electronic Journal of Biotechnology 2009, 12 (4).

6. Zhang, H.; Mu, W. H.; Hou, Z. G.; Wu, X.; Zhao, W. W.; Zhang, X. H.; Pan, H. Y.; Zhang, S. H., Biodegradation of Nicosulfuron by the Bacterium Serratia marcescens N80. Journal of Environmental Science and Health Part BPesticides Food Contaminants and Agricultural Wastes 2012, 47 (3), 153-160.

7. Rafii, F.; Franklin, W.; Heflich, R. H.; Cerniglia, C. E., Reduction of Nitroaromatic Compounds by Anaerobic-Bacteria Isolated from the Human GastrointestinalTract. Applied and Environmental Microbiology 1991, 57 (4), 962-968.

8. Rafii, F.; Cerniglia, C. E., Comparison of the Azoreductase and Nitroreductase from Clostridium perfringens. Applied and Environmental Microbiology 1993, 59 (6), 1731-1734. 
9. Rafii, F.; Cerniglia, C. E., Reduction of Azo Dyes and Nitroaromatic Compounds by Bacterial Enzymes from the Human Intestinal Tract. Environmental Health Perspectives 1995, 103, 17-19.

10. Manning, B. W.; Campbell, W. L.; Franklin, W.; Delclos, K. B.; Cerniglia, C. E., Metabolism of 6-Nitrochrysene by Intestinal Microflora. Applied and Environmental Microbiology 1988, 54 (1), 197-203.

11. Gallagher, E. M.; Young, L. Y.; McGuinness, L. M.; Kerkhof, L. J., Detection of 2,4,6-Trinitrotoluene-Utilizing Anaerobic Bacteria by (15) N and (13)C Incorporation. Applied and Environmental Microbiology 2010, 76 (5), 1695-1698.

12. Nishino, S. F.; Paoli, G. C.; Spain, J. C., Aerobic Degradation of Dinitrotoluenes and Pathway for Bacterial Degradation of 2,6-dinitrotoluene. Applied and Environmental Microbiology 2000, 66 (5), 2139-2147.

13. Kuntze, K.; Kiefer, P.; Baumann, S.; Seifert, J.; von Bergen, M.; Vorholt, J. A.; Boll, M., Enzymes Involved in the Anaerobic Degradation of Meta-Substituted Halobenzoates. Molecular Microbiology 2011, 82 (3), 758-769.

14. Peppercorn, M.; Goldman, P., Distribution Studies of Salicylazosulfapyridine and Its Metabolites. Gastroenterology 1973, 64 (2), 240-245.

15. Koch, R. L.; Goldman, P., Anaerobic Metabolism of Metronidazle Forms N-(2Hydroxyethyl)-Oxamic Acid. Journal of Pharmacology and Experimental Therapeutics 1979, 208 (3), 406-410.

16. Robertson, M. D.; Drummer, O. H., Postmortem Drug-Metabolism by Bacteria. Journal of Forensic Sciences 1995, 40 (3), 382-386.

17. Robertson, M. D.; Drummer, O. H., High-Performance Liquid-Chromatographic Procedure for the Measurement of Nitrobenzodiazepines and Their 7-Amino Metabolites in Blood. Journal of Chromatography B-Biomedical Applications 1995, 667 (1), 179-184. 
18. Bresler, M. M.; Rosser, S. J.; Basran, A.; Bruce, N. C., Gene Cloning and Nucleotide Sequencing and Properties of a Cocaine Esterase from Rhodococcus sp. strain MB1. Applied and Environmental Microbiology 2000, 66 (3), 904-908.

19. Britt, A. J.; Bruce, N. C.; Lowe, C. R., Identification of a Cocaine Esterase in a Strain of Pseudomonas maltophilia. Journal of Bacteriology 1992, 174 (7), 2087 2094.

20. Brim, R. L.; Nance, M. R.; Youngstrom, D. W.; Narasimhan, D.; Zhan, C.-G.; Tesmer, J. J. G.; Sunahara, R. K.; Woods, J. H., A Thermally Stable Form of Bacterial Cocaine Esterase: A Potential Therapeutic Agent for Treatment of Cocaine Abuse. Molecular Pharmacology 2010, 77 (4), 593-600.

21. Brim, R. L.; Noon, K. R.; Collins, G. T.; Nichols, J.; Narasimhan, D.; Sunahara, R. K.; Woods, J. H., The Ability of Bacterial Cocaine Esterase to Hydrolyze Cocaine Metabolites and Their Simultaneous Quantification Using High-Performance Liquid Chromatography-Tandem Mass Spectrometry. Molecular Pharmacology 2011, 80 (6), 1119-1127.

22. Labroo, R. B.; Paine, M. F.; Thummel, K. E.; Kharasch, E. D., Fentanyl Metabolism by Human Hepatic and Intestinal Cytochrome P450 3A4: Implications for Interindividual Variability in Disposition, Efficacy, and Drug Interactions. Drug Metabolism and Disposition 1997, 25 (9), 1072-1080.

23. Cameron, G. W. W.; Jordan, K. N.; Holt, P. J.; Baker, P. B.; Lowe, C. R.; Bruce, N. C., Identification of a Heroin Esterase in Rhodococcus sp. Strain H1. Applied and Environmental Microbiology 1994, 60 (10), 3881-3883.

24. Drug Enforcement Administration and U.S. Department of Justice. Drugs of Abuse. http://www.justice.gov/dea/druginfo/factsheets.shtml (accessed November 25, 2013.). 
25. International Conference on Harmonisation. Validation of Analytical Procedures: Text and Methodology Q2(R1).

http://www.ich.org/fileadmin/Public Web Site/lCH Products/Guidelines/Quality/ Q2 R1/Step4/Q2 R1 Guideline.pdf (accessed December 3, 2013).

26. Scientific Working Group for Forensic Toxicology (SWGTOX) Standard Practices for Method Validation in Forensic Toxicology. Journal of Analytical Toxicology 2013, 37 (7), 452-474.

27. US Department of Health and Human Services, Food and Drug Administration, Center for Drug Evaluation and Research, Center for Veterinary Medicine. Guidance for Industry: Bioanalytical Method Validation.

http://www.fda.gov/downloads/Drugs/GuidanceComplianceRegulatorylnformation /Guidances/ucm070107.pdf (accessed December 3, 2013).

28. Matuszewski, B. K.; Constanzer, M. L.; Chavez-Eng, C. M., Strategies for the Assessment of Matrix Effect in Quantitative Bioanalytical Methods Based on HPLC-MS/MS. Analytical Chemistry 2003, 75 (13), 3019-3030.

29. Vass, A. After a Person's Pulse and Breathing Stop, How Much Later Does All Cellular Metabolism Stop? Scientific American [Online], 2007. http://www.scientificamerican.com/article.cfm?id=experts-cell-metabolism-afterdeath. (accessed December 3, 2013).

30. Vass, A. A.; Barshick, S. A.; Sega, G.; Caton, J.; Skeen, J. T.; Love, J. C.; Synstelien, J. A., Decomposition Chemistry of Human Remains: A New Methodology for Determining the Postmortem Interval. Journal of Forensic Sciences 2002, 47 (3), 542-553.

31. Vass, A. A., Beyond the Grave - Understanding Human Decomposition. Microbiology Today: 1994; Vol. 28, pp 190-192. 
32. Bowen, R. Microbial Life in the Digestive Tract.

http://www.vivo.colostate.edu/hbooks/pathphys/digestion/basics/gi bugs.html (accessed November 1, 2013).

33. Hentges, D. J., The Anaerobic Micrfoflora of the Human Body. Clinical Infectious Diseases 1993, 16, S175-S180.

34. Tortora, G. J.; Berdell, R. F.; Case, C. L., Microbiology: An Introduction. 9th ed.; Benjamin Cummings: 2006.

35. Bergey's Manual of Systematic Bacteriology, Vol 4, Second Edition. Springer: 2011.

36. Cappucino, J.; Sherman, N., Microbiology: A Laborastory Manual. 9th ed.; Pearson Education: 2011.

37. Melvin, J. R.; Cronholm, L. S.; Simson, L. R.; Isaacs, A. M., Bacterial Transmigration as an Indicator of the Time of Death. Journal of Forensic Sciences 1984, 29 (2), 412-417.

38. American Type Culture Collection. http://www.atcc.org/en/Documents/Learning Center/Material Safety Data Shee ts.aspx (accessed December 3, 2013).

39. Centers for Disease Control - Laboratory Biosafety Level Criteria. http://www.cdc.gov/biosafety/publications/bmbl5/bmbl5 sect iv.pdf (accessed December 3, 2013.).

40. $B D$ Difco ${ }^{\mathrm{TM}} \& B B L^{\mathrm{TM}}$ Manual, 2nd Edition: Reinforced Clostridial Medium. www.bd.com/europe/regulatory/Assets/IFU/Difco BBL/218081.pdf (accessed October 5).

41. Dixon, M., Edgewood Chemical Biological Center Wolin Salts and M9 Medium Preparation. Martindale, S., Ed. p 2. 
42. Collaborative Institutional Training Initiative $(\mathrm{CITI})$ at the University of Miami Biosafety and Biosecurity. https://www.citiprogram.org/index.cfm?pagelD=86 (accessed December 3, 2013.).

43. Centers for Disease Control and Prevention - Biosafety. http://www.cdc.gov/biosafety/ (accessed December 3, 2013.).

44. Coy Laboratory Products, INC. Anaerobic Chambers. www.tmaapr.com/pdf/COY Product Catalog Anaerobic 911.pdf (accessed December 3, 2013.).

45. National Institutes of Health Publication No. 08-2681 - Your Digestive System and How It Works. 2008.

46. Jones, A. W.; Holmgren, A., Concentration Distributions of the Drugs Most Frequently Identified in Post-Mortem Femoral Blood Representing all Causes of Death. Medicine Science and the Law 2009, 49 (4), 257-273.

47. Marchi, I.; Schappler, J.; Veuthey, J.-L.; Rudaz, S., Development and Validation of a Liquid Chromatography-Atmospheric Pressure Photoionization-Mass Spectrometry Method for the Quantification of Alprazolam, Flunitrazepam, and Their Main Metabolites in Haemolysed Blood. Journal of Chromatography BAnalytical Technologies in the Biomedical and Life Sciences 2009, 877 (23), 2275-2283.

48. Sauve, E. N.; Langodegard, M.; Ekeberg, D.; Oiestad, A. M. L., Determination of Benzodiazepines in Ante-Mortem and Post-Mortem Whole Blood by SolidSupported Liquid-Liquid Extraction and UPLC-MS/MS. Journal of Chromatography B-Analytical Technologies in the Biomedical and Life Sciences 2012, 883, 177-188. 
49. Liu, Z.; Short, J.; Rose, A.; Ren, S.; Contel, N.; Grossman, S.; Unger, S., The Simultaneous Determination of Diazepam and Its Three Metabolites in Dog Plasma by High-Performance Liquid Chromatography with Mass Spectroscopy Detection. Journal of Pharmaceutical and Biomedical Analysis 2001, 26 (2), 321 330.

50. Miller, E. I.; Wylie, F. A.; Oliver, J. S., Simultaneous Detection and Quantification of Amphetamines, Diazepam and Its Metabolites, Cocaine and Its Metabolites, and Opiates in Hair by LC-ESI-MS-MS using a Single Extraction Method. Journal of Analytical Toxicology 2008, 32 (7), 457-469.

51. Lendoiro, E.; Quintela, O.; de Castro, A.; Cruz, A.; Lopez-Rivadulla, M.; Concheiro, M., Target Screening and Confirmation of 35 Licit and Illicit Drugs and Metabolites in Hair by LC-MSMS. Forensic Science International 2012, 217 (1-3), 207-215.

52. Umezawa, H.; Lee, X.-P.; Arima, Y.; Hasegawa, C.; Marumo, A.; Kumazawa, T.; Sato, K., Determination of Diazepam and Its Metabolites in Human Urine by Liquid Chromatography/Tandem Mass Spectrometry Using a Hydrophilic Polymer Column. Rapid Communications in Mass Spectrometry 2008, 22 (15), 2333-2341.

53. Jourdil, N.; Bessard, J.; Vincent, F.; Eysseric, H.; Bessard, G., Automated SolidPhase Extraction and Liquid Chromatography-Electrospray lonization-Mass Spectrometry for the Determination of Flunitrazepam and Its Metabolites in Human Urine and Plasma Samples. Journal of Chromatography B-Analytical Technologies in the Biomedical and Life Sciences 2003, 788 (2), 207-219.

54. DOJ; DEA Drugs of Abuse. http://www.justice.gov/dea/druginfo/factsheets.shtml (accessed October 5). 
55. National Highway Traffic Safety Administration - Drugs and Human Performance Fact Sheets: Diazepam.

http://www.nhtsa.gov/people/injury/research/job185drugs/diazepam.htm (accessed December 3, 2013).

56. Smith-Kielland, A.; Skuterud, B.; Olsen, K. M.; Morland, J., Urinary excretion of diazepam metabolites in healthy volunteers and drug users. Scandinavian Journal of Clinical \& Laboratory Investigation 2001, 61 (3), 237-246.

57. Kilicarslan, T.; Haining, R. L.; Rettie, A. E.; Busto, U.; Tyndale, R. F.; Sellers, E. M., Flunitrazepam Metabolism by Cytochrome P450S2C19 and 3A4. Drug Metabolism and Disposition 2001, 29 (4), 460-465.

58. Malanciuc, C.; Arama, C.; Saramet, I.; Monciu, C.-M.; Nedelcu, A.; Constantinescu, C., Analytical Characterization of Flunitrazepam. Farmacia 2009, 57 (2), 167-183.

59. LeBeau, M. A.; Montgomery, M. A.; Wagner, J. R.; Miller, M. L., Analysis of Biofluids for Flunitrazepam and Metabolites by Electrospray Liquid Chromatography/Mass Spectrometry. Journal of Forensic Sciences 2000, 45 (5), 1133-1141.

60. Nakamura, M., Analyses of benzodiazepines and their metabolites in various biological matrices by LC-MS(/MS). Biomedical Chromatography 2011, 25 (12), 1283-1307.

61. Kintz, P.; Villain, M.; Concheiro, M.; Cirimele, V., Screening and Confirmatory Method for Benzodiazepines and Hypnotics in Oral Fluid by LC-MS/MS. Forensic Science International 2005, 150 (2-3), 213-220.

62. Kempf, J.; Wuske, T.; Schubert, R.; Weinmann, W., Pre-Analytical Stability of Selected Benzodiazepines on a Polymeric Oral Fluid Sampling Device. Forensic Science International 2009, 186 (1-3), 81-85. 
63. Glennon, R. A.; Young, R., Drug Discrimination : Applications to Medicinal Chemistry and Drug Studies. Wiley: Hoboken, New Jersey, 2011; p xx, 510 pages.

64. Substance Abuse and Mental Health Services Administration - National Survey on Drug Use and Health.

http://www.samhsa.gov/data/NSDUH/2012SummNatFindDetTables/Index.aspx. (accessed December 3, 2013).

65. Bijlsma, L.; Sancho, J. V.; Hernandez, F.; Niessen, W. M. A., Fragmentation Pathways of Drugs of Abuse and Their Metabolites Based on QTOF MS/MS and MSE Accurate-Mass Spectra. Journal of Mass Spectrometry 2011, 46 (9), 865875;

66. Cone, E. J.; Sampson-Cone, A. H.; Darwin, W. D.; Huestis, M. A.; Oyler, J. M., Urine Testing for Cocaine Abuse: Metabolic and Excretion Patterns following Different Routes of Administration and Methods for Detection of False-Negative Results. Journal of Analytical Toxicology 2003, 27 (7), 386-401.

67. Xia, Y.; Wang, P. P.; Bartlett, M. G.; Solomon, H. M.; Busch, K. L., An LC-MS-MS Method for the Comprehensive Analysis of Cocaine and Cocaine Metabolites in Meconium. Analytical Chemistry 2000, 72 (4), 764-771.

68. Zhang, J. Y.; Foltz, R. L., Cocaine Metabolism in Man - Identification of 4 Previously Unreported Cocaine Metabolites in Human Urine. Journal of Analytical Toxicology 1990, 14 (4), 201-205.

69. Bonate, P. L.; Davis, C. M.; Silverman, P. B.; Swann, A., Determination of Cocaine in Biological Matrices using Reversed-Phase HPLC - Application to Plasma and Brain-Tissue. Journal of Liquid Chromatography 1995, 18 (17), 3473-3494. 
70. Concheiro, M.; Gray, T. R.; Shakleya, D. M.; Huestis, M. A., High-Throughput Simultaneous Analysis of Buprenorphine, Methadone, Cocaine, Opiates, Nicotine, and Metabolites in Oral Fluid by Liquid Chromatography Tandem Mass Spectrometry. Analytical and Bioanalytical Chemistry 2010, 398 (2).

71. de Castro, A.; Concheiro, M.; Shakleya, D. M.; Huestis, M. A., Simultaneous Quantification of Methadone, Cocaine, Opiates, and Metabolites in Human Placenta by Liquid Chromatography-Mass Spectrometry. Journal of Analytical Toxicology 2009, 33 (5), 243-252.

72. EISohly, M. A.; Kopycki, W.; Feng, S. X.; Murphy, T. P., Identification and Analysis of the Major Metabolites of Cocaine in Meconium. Journal of Analytical Toxicology 1999, 23 (6), 446-451.

73. Gunn, J.; Kriger, S.; Terrell, A. R., Detection and Quantification of Cocaine and Benzoylecgonine in Meconium Using Solid Phase Extraction and UPLC/MS/MS. Clinical Applications of Mass Spectrometry:METHODS AND PROTOCOLS 2010, 603, 165-174.

74. Jagerdeo, E.; Montgomery, M. A.; LeBeau, M. A.; Sibum, M., An Automated SPE/LC/MS/MS Method for the Analysis of Cocaine and Metabolites in Whole Blood. Journal of Chromatography B-Analytical Technologies in the Biomedical and Life Sciences 2008, 874 (1-2).

75. Johansen, S. S.; Bhatia, H. M., Quantitative Analysis of Cocaine and Its Metabolites in Whole Blood and Urine by High-Performance Liquid Chromatography Coupled with Tandem Mass Spectrometry. Journal of Chromatography B-Analytical Technologies in the Biomedical and Life Sciences 2007, 852 (1-2), 338-344.

76. Jones, R. T., Pharmacokinetics of Cocaine: Considerations when Assessing Cocaine Use by Urinalysis. NIDA research monograph 1997, 175, 221-34. 
77. Klingmann, A.; Skopp, G.; Aderjan, R., Analysis of Cocaine, Benzoylecgonine, Ecgonine Methyl Ester, and Ecgonine by High-Pressure Liquid ChromatographyAPI Mass Spectrometry and Application to a Short-Term Degradation Study of Cocaine in Plasma. Journal of Analytical Toxicology 2001, 25 (6), 425-430.

78. Kolbrich, E. A.; Barnes, A. J.; Gorelick, D. A.; Boyd, S. J.; Cone, E. J.; Huestis, M. A., Major and Minor Metabolites of Cocaine in Human Plasma Following Controlled Subcutaneous Cocaine Administration. Journal of Analytical Toxicology 2006, 30 (8), 501-510.

79. Popa, D.-S.; Vlase, L.; Leucuta, S. E.; Loghin, F., Determination of Cocaine and Benzoylecgonine in Human Plasma by LC-MS/MS. Farmacia 2009, 57 (3), 301308.

80. Shakleya, D. M.; Huestis, M. A., Simultaneous Quantification of Nicotine, Opioids, Cocaine, and Metabolites in Human Fetal Postmortem Brain by Liquid Chromatography Tandem Mass Spectrometry. Analytical and Bioanalytical Chemistry 2009, 393 (8), 1957-1965.

81. Srinivasan, K.; Wang, P.; Eley, A. T.; White, C. A.; Bartlett, M. G., Liquid Chromatography-Tandem Mass Spectrometry Analysis of Cocaine and Its Metabolites from Blood, Amniotic Fluid, Placental and Fetal Tissues: Study of the Metabolism and Distribution of Cocaine in Pregnant Rats. Journal of Chromatography B 2000, 745 (2), 287-303.

82. Justice, U. S. D. o.; Administration, D. E. Drugs of Abuse. http://www.justice.gov/dea/druginfo/factsheets.shtml (accessed October 5).

83. Coopman, V.; Cordonnier, J.; Pien, K.; Van Varenbergh, D., LC-MS/MS Analysis of Fentanyl and Norfentanyl in a Fatality Due to Application of Multiple Durogesic (R) Transdermal Therapeutic Systems. Forensic Science International 2007, 169 (2-3), 223-227. 
84. Clavijo, C. F.; Thomas, J. J.; Cromie, M.; Schniedewind, B.; Hoffman, K. L.; Christians, U.; Galinkin, J. L., A Low Blood Volume LC-MS/MS Assay for the Quantification of Fentanyl and Its Major Metabolites Norfentanyl and Despropionyl Fentanyl in Children. Journal of Separation Science 2011, 34 (24), 3568-3577.

85. Olson, K. N.; Luckenbill, K.; Thompson, J.; Middleton, O.; Geiselhart, R.; Mills, K. M.; Kloss, J.; Apple, F. S., Postmortem Redistribution of Fentanyl in Blood. American Journal of Clinical Pathology 2010, 133 (3), 447-453.

86. Day, J.; Slawson, M.; Lugo, R. A.; Wilkins, D., Analysis of Fentanyl and Norfentanyl in Human Plasma by Liquid Chromatography-Tandem Mass Spectrometry using Electrospray Ionization. Journal of Analytical Toxicology 2003, 27 (7), 513-516.

87. Thevis, M.; Geyer, H.; Bahr, D.; Schanzer, W., Identification of Fentanyl, Alfentanil, Sufentanil, Remifentanil and Their Major Metabolites in Human Urine by Liquid Chromatography/Tandem Mass Spectrometry for Doping Control Purposes. European Journal of Mass Spectrometry 2005, 11 (4), 419-427.

88. Verplaetse, R.; Tytgat, J., Development and Validation of a Sensitive UPLCMS/MS Method for the Analysis of Narcotic Analgesics in Urine and Whole Blood in Forensic Context. Forensic Science International 2012, 215 (1-3), 136-145.

89. DHHS; FDA; CDER; CVM US Department of Health and Human Services, Food and Drug Administration, Center for Drug Evaluation and Research, Center for Veterinary Medicine. Guidance for Industry: Bioanalytical Method Validation. http://www.fda.gov/downloads/Drugs/GuidanceComplianceRegulatorylnformation /Guidances/ucm070107.pdf (accessed December 3, 2013).

90. Caplehorn, J. R. M.; Drummer, O. H., Methadone Dose and Post-Mortem Blood Concentration. Drug and Alcohol Review 2002, 21 (4), 329-333. 
91. Choo, R. E.; Jansson, L. M.; Scheidweiler, K.; Huestis, M. A., A Validated Liquid Chromatography-Atmospheric Pressure Chemical Ionization-Tandem Mass Spectrometric Method for the Quantification of Methadone, 2-ethylidene-1,5dimethyl-3,3-diphenylpyrrolidine (EDDP), and 2-ethyl-5-methyl-3,3diphenylpyroline (EMDP) in Human Breast Milk. Journal of Analytical Toxicology 2007, 31 (5), 265-269.

92. Drummer, O. H., Postmortem Toxicology of Drugs of Abuse. Forensic Science International 2004, 142 (2-3), 101-113.

93. Goldberger, B. A.; Darraj, A. G.; Caplan, Y. H.; Cone, E. J., Detection of Methadone, Methadone Metabolites, and Other Illicit Drugs of Abuse in Hair of Methadone-Treatment Subjects. Journal of Analytical Toxicology 1998, 22 (6), 526-530.

94. Kintz, P.; Villain, M.; Dumestre-Toulet, V.; Capolaghi, B.; Cirimele, V., Methadone as a Chemical Weapon - Two Fatal Cases Involving Babies. Therapeutic Drug Monitoring 2005, 27 (6), 741-743.

95. Maurer, H. H., Advances in Analytical Toxicology: the Current Role of Liquid Chromatography-Mass Spectrometry in drug Quantification in Blood and Oral Fluid. Analytical and Bioanalytical Chemistry 2005, 381 (1), 110-118.

96. Nelson, P. E.; Selkirk, R. C., The Toxicology of 12 Cases of Death Involving Methadone Examination of Post Mortem Specimens. Forensic Science 1975, 6 (3), 175-186.

97. Rook, E. J.; Hillebrand, M. J. X.; Rosing, H.; van Ree, J. M.; Beijnen, J. H., The Quantitative Analysis of Heroin, Methadone and Their Metabolites and the Simultaneous Detection of Cocaine, Acetylcodeine and Their Metabolites in Human Plasma by High-Performance Liquid Chromatography Coupled with Tandem Mass Spectrometry. Journal of Chromatography B-Analytical Technologies in the Biomedical and Life Sciences 2005, 824 (1-2), 213-221. 
98. Caldwell, J.; Dring, L. G.; Williams, R. T., Metabolism of C-14 Methamphetamine in Man, Guinea-Pig and Rat. Biochemical Journal 1972, 129 (1), 11-\&.

99. Fuh, M. R. S.; Lu, K. T., Determination of Amphetamine and Methamphetamine in Human Urine by Electrospray Mass Spectrometry. Abstracts of Papers of the American Chemical Society 1997, 213, 61-ANYL.

100. Fuh, M. R.; Wu, T. Y.; Lin, T. Y., Determination of Amphetamine and Methamphetamine in Urine by Solid Phase Extraction and Ion-Pair Liquid Chromatography-Electrospray-Tandem Mass Spectrometry. Talanta 2006, 68 (3), 987-991.

101. Kuwayama, K.; Inoue, H.; Kanamori, T.; Tsujikawa, K.; Miyaguchi, H.; Iwata, Y. T.; Miyauchi, S.; Kamo, N., Analysis of Amphetamine-Type Stimulants and Their Metabolites in Plasma, Urine and Bile by Liquid Chromatography with a Strong Cation-Exchange Column-Tandem Mass Spectrometry. Journal of Chromatography B-Analytical Technologies in the Biomedical and Life Sciences 2008, 867 (1), 78-83.

102. Shima, N.; Kamata, H. T.; Katagi, M.; Tsuchihashi, H., Urinary Excretion of the Main Metabolites of Methamphetamine, Including p-HydroxymethamphetamineSulfate and p-Hydroxymethamphetamine-Glucuronide, in Humans and Rats. Xenobiotica 2006, 36 (2-3), 259-267. 


\section{Appendix A - Calibration Curves for Diazepam, Flunitrazepam, and Metabolites in Buffer}

\section{Diazepam}

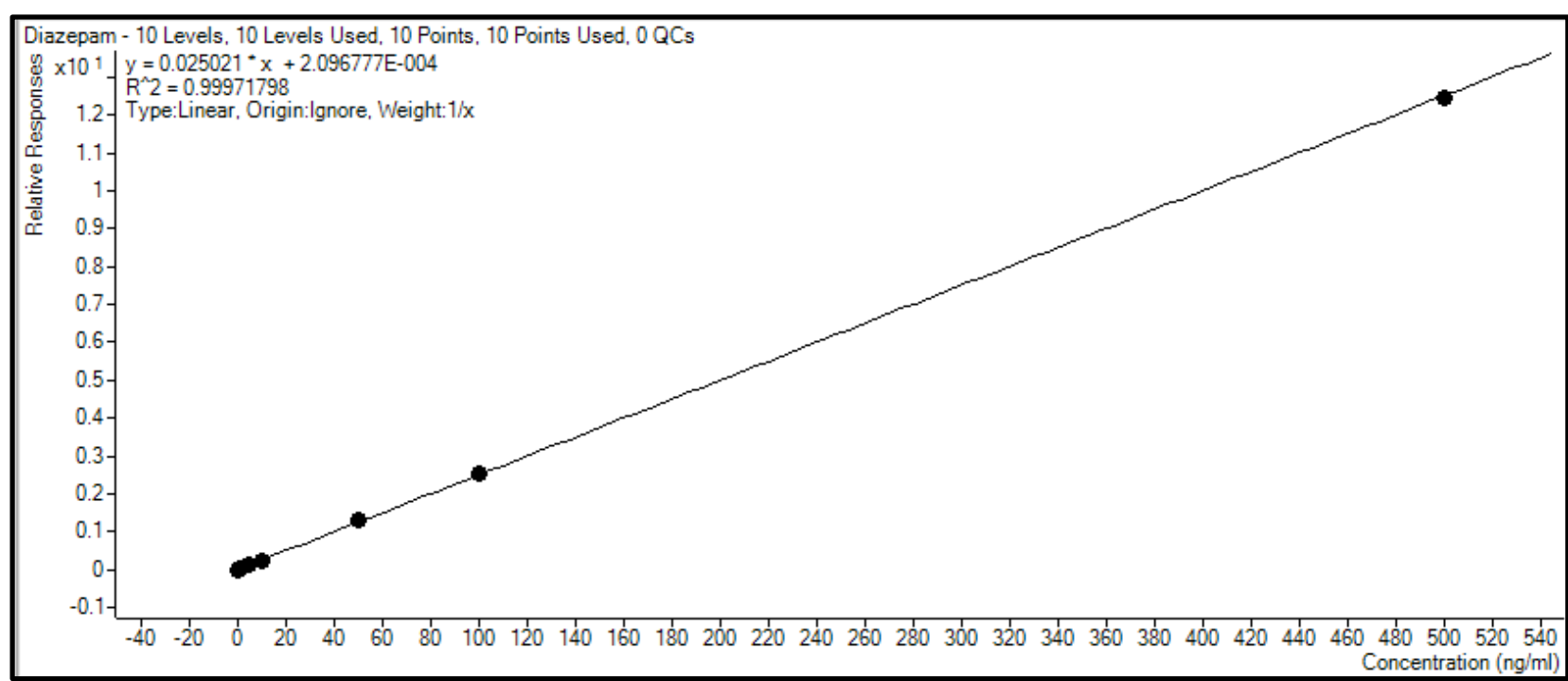

\section{Nordiazepam}

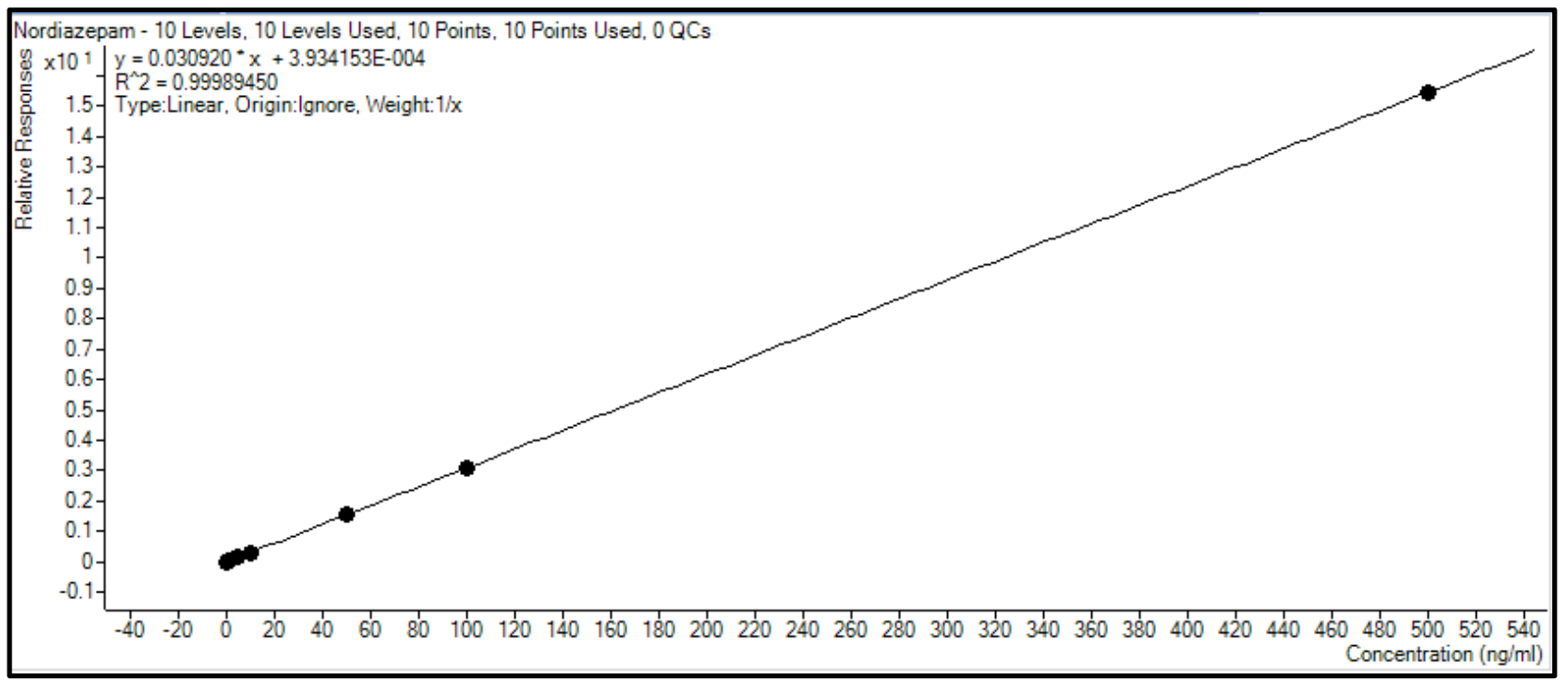




\section{Oxazepam}

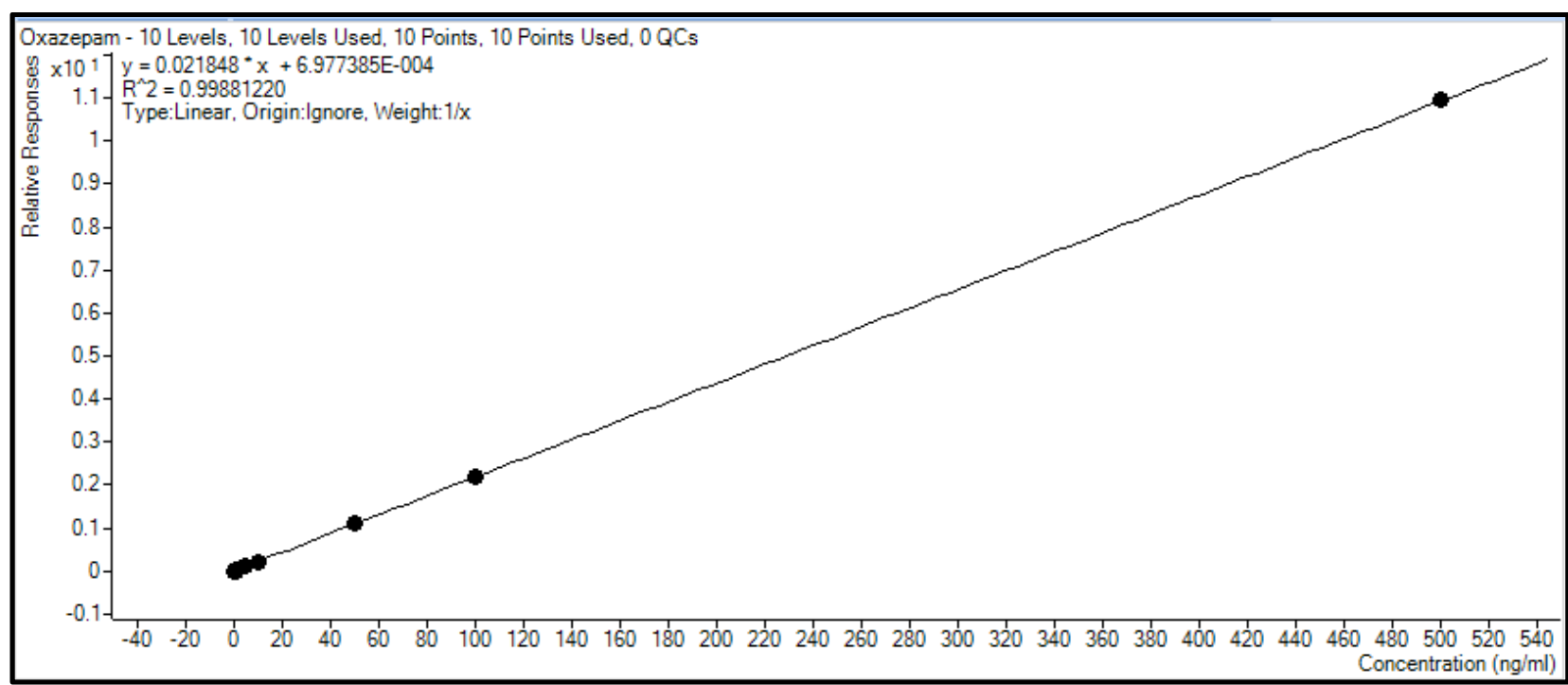

\section{Temazepam}

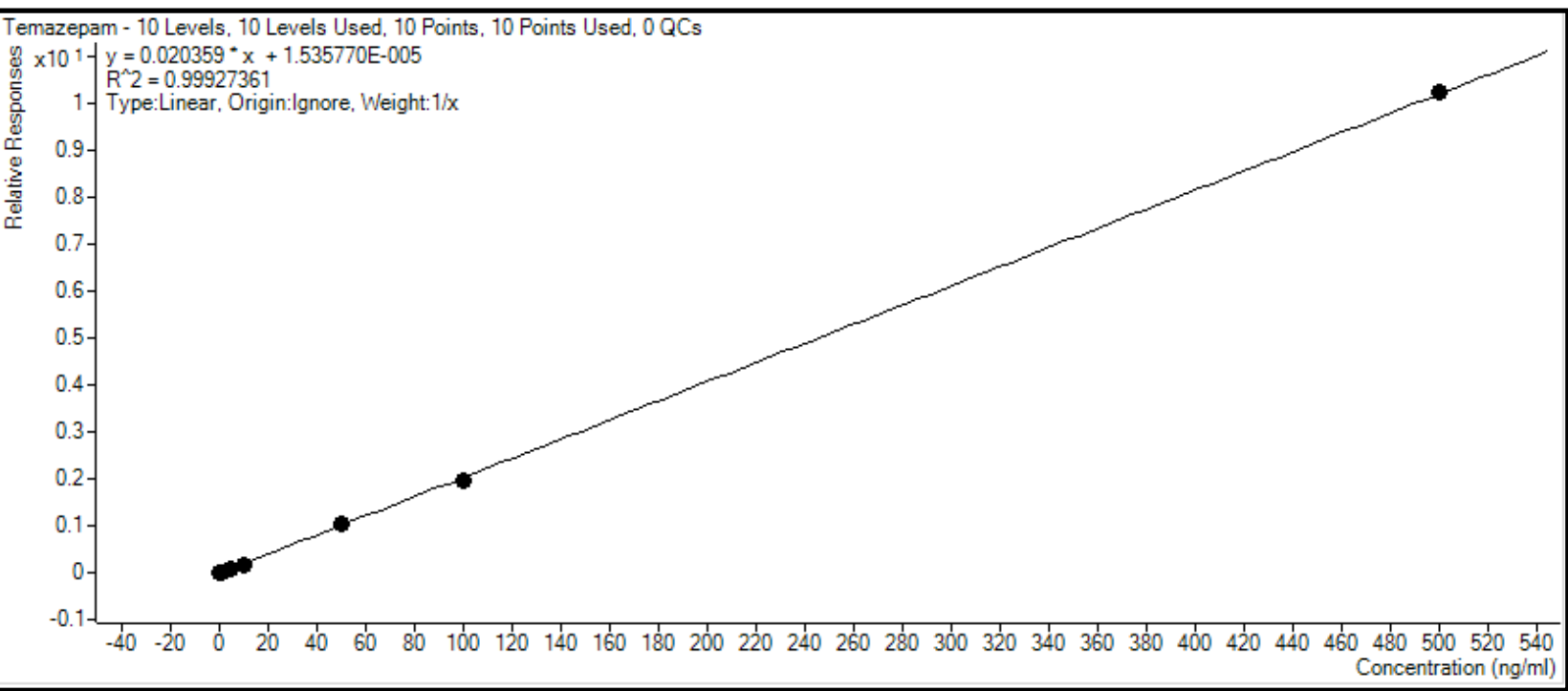




\section{Flunitrazepam}

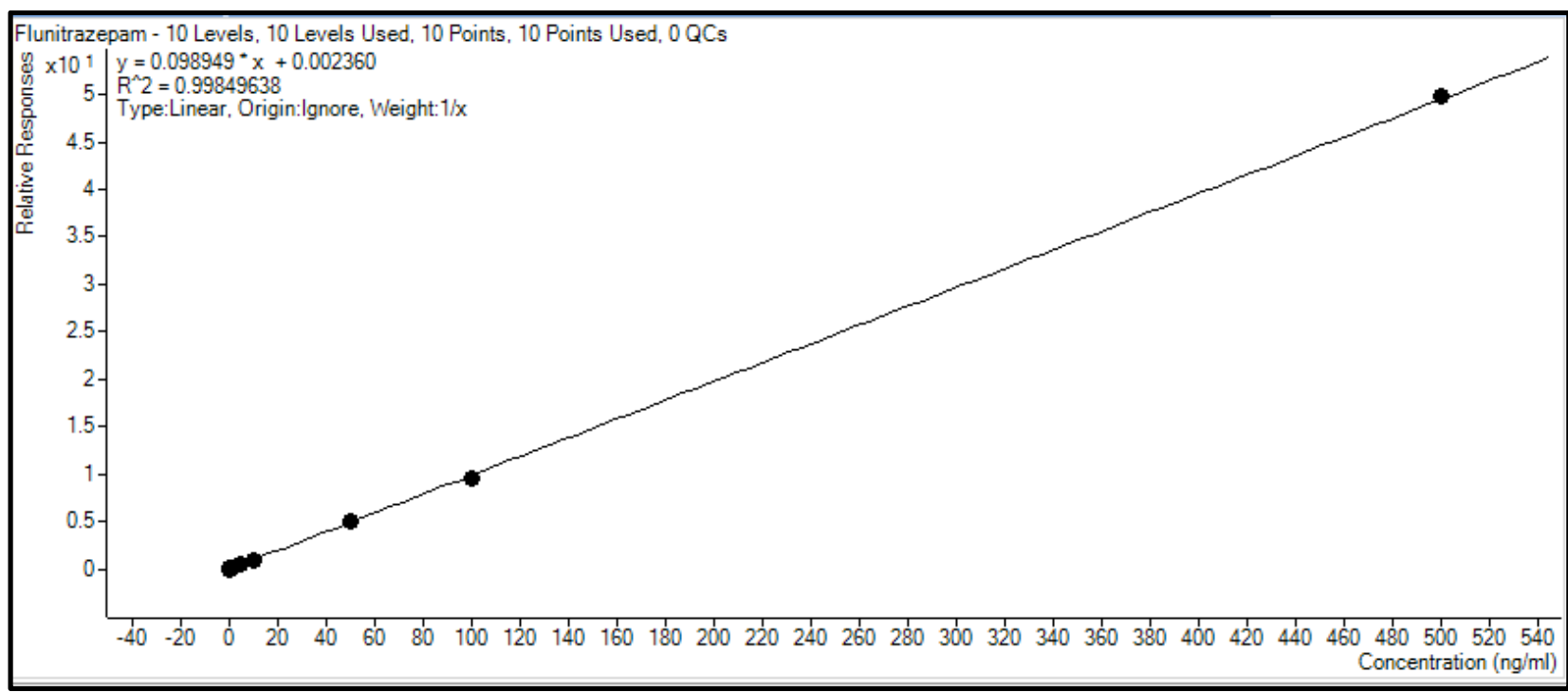

\section{7-Aminoflunitrazepam}

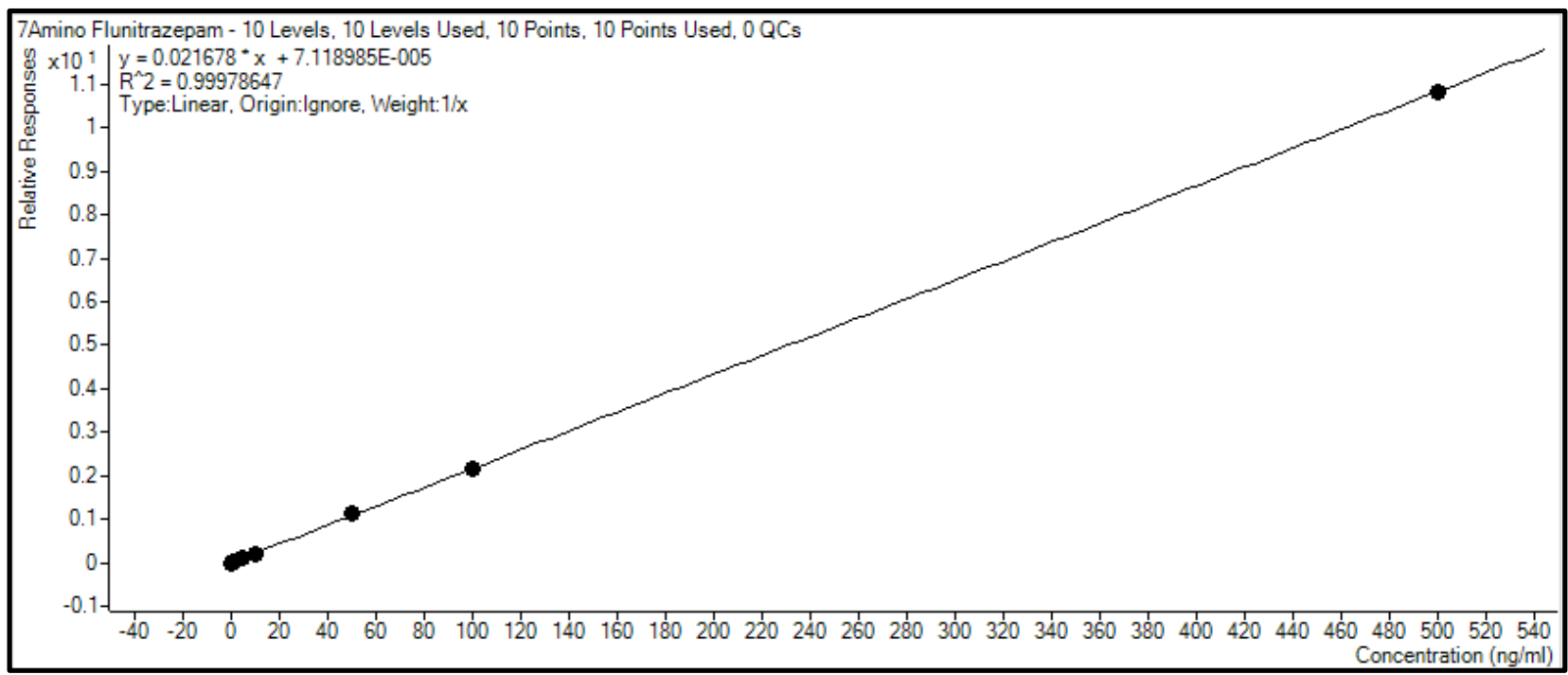




\section{$\mathrm{N}$-Desmethylflunitrazepam}

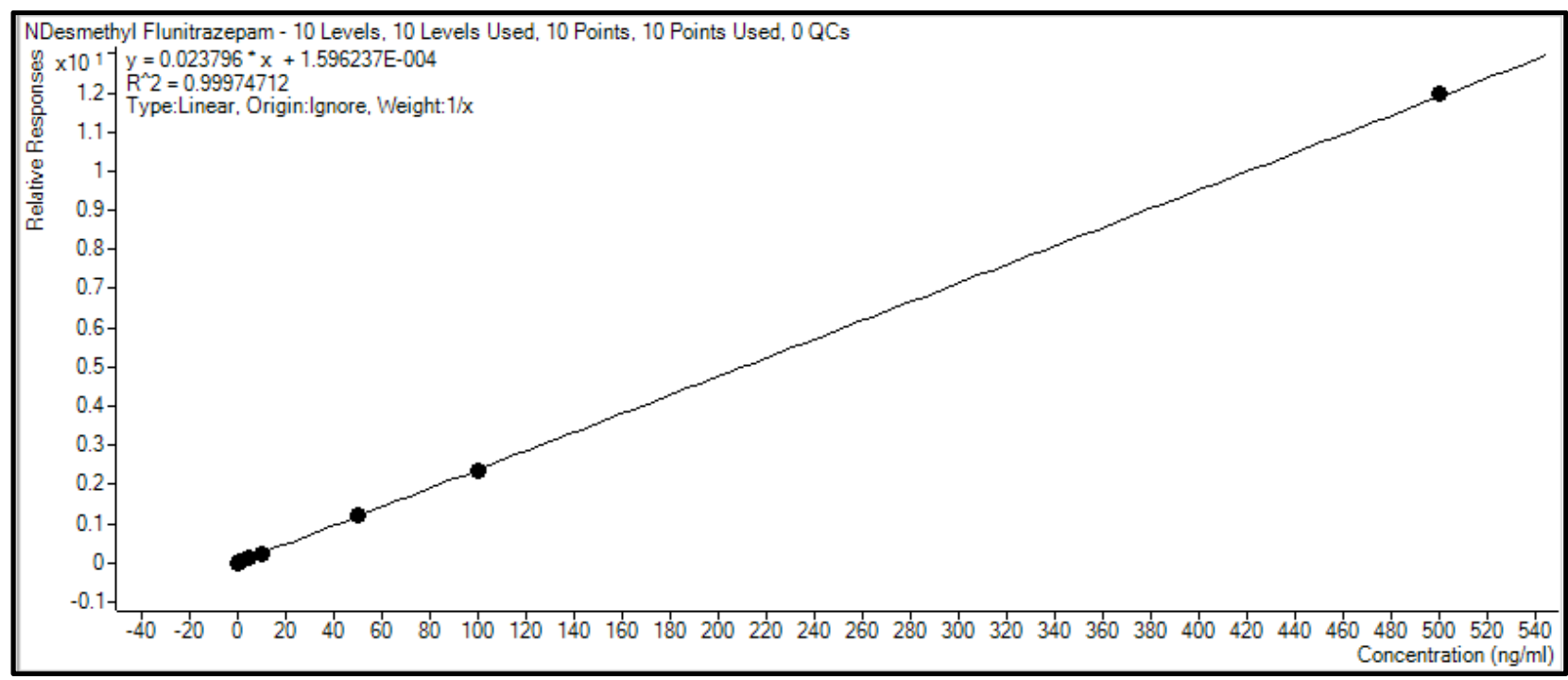




\section{Appendix B - Calibration Curves for Diazepam, Flunitrazepam, and Metabolites in Media}

\section{Diazepam}

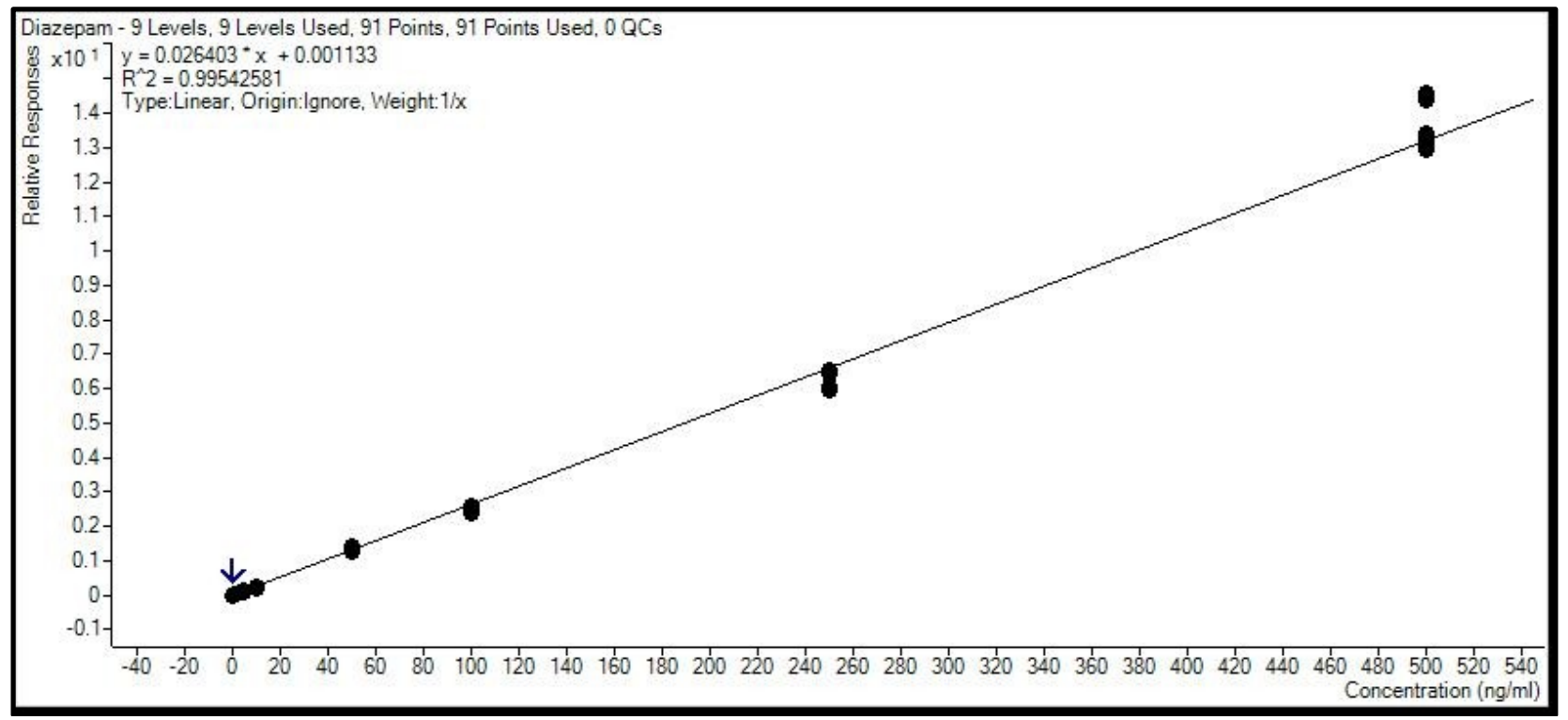

\section{Nordiazepam}

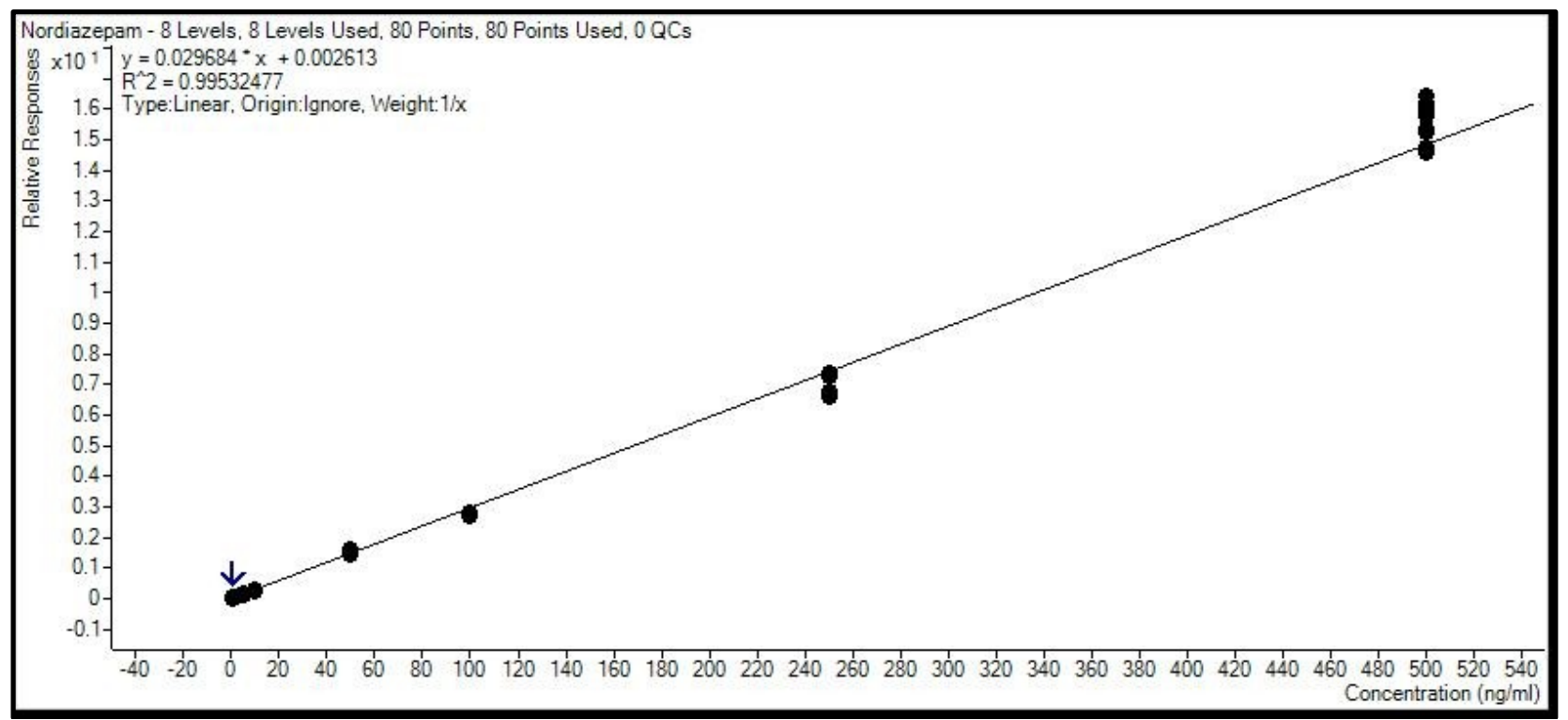




\section{Oxazepam}

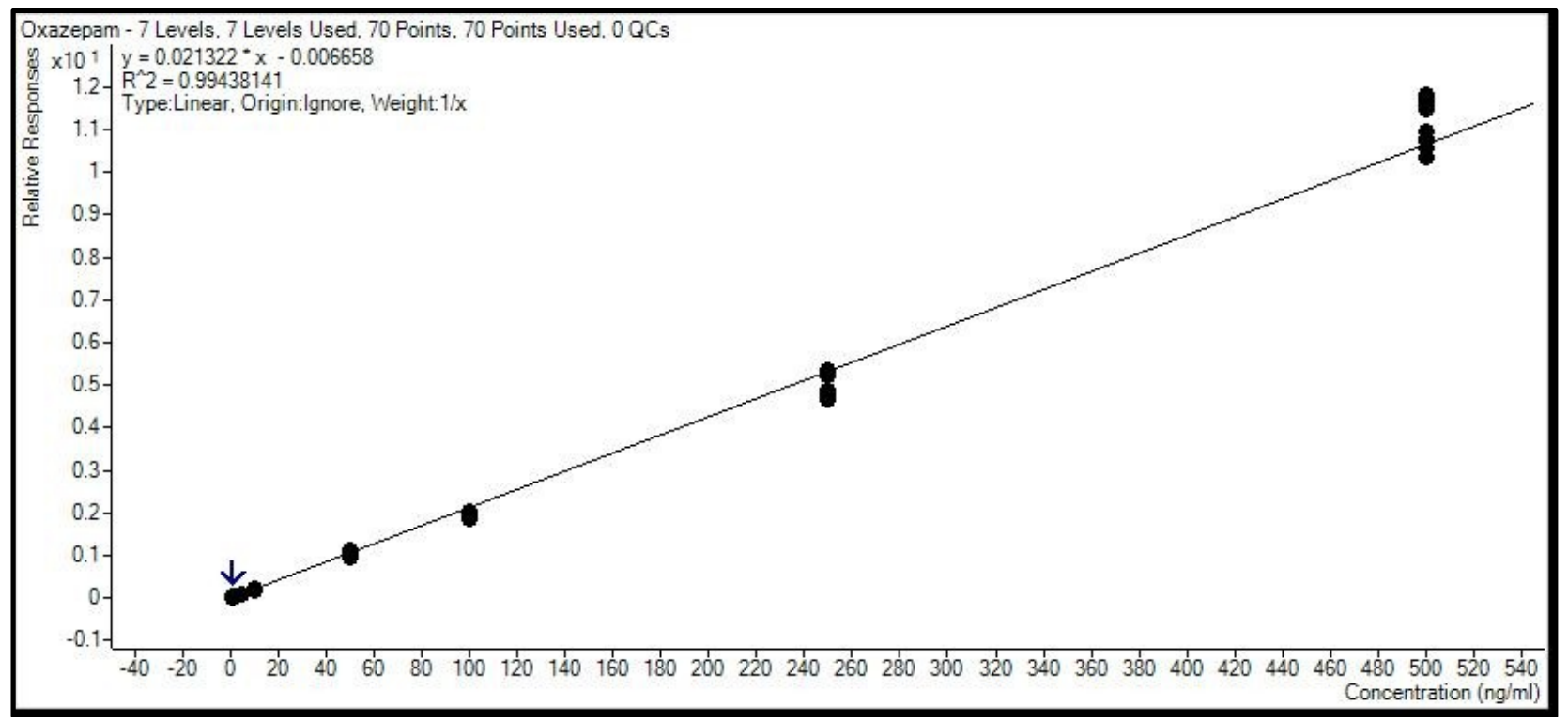

\section{Temazepam}

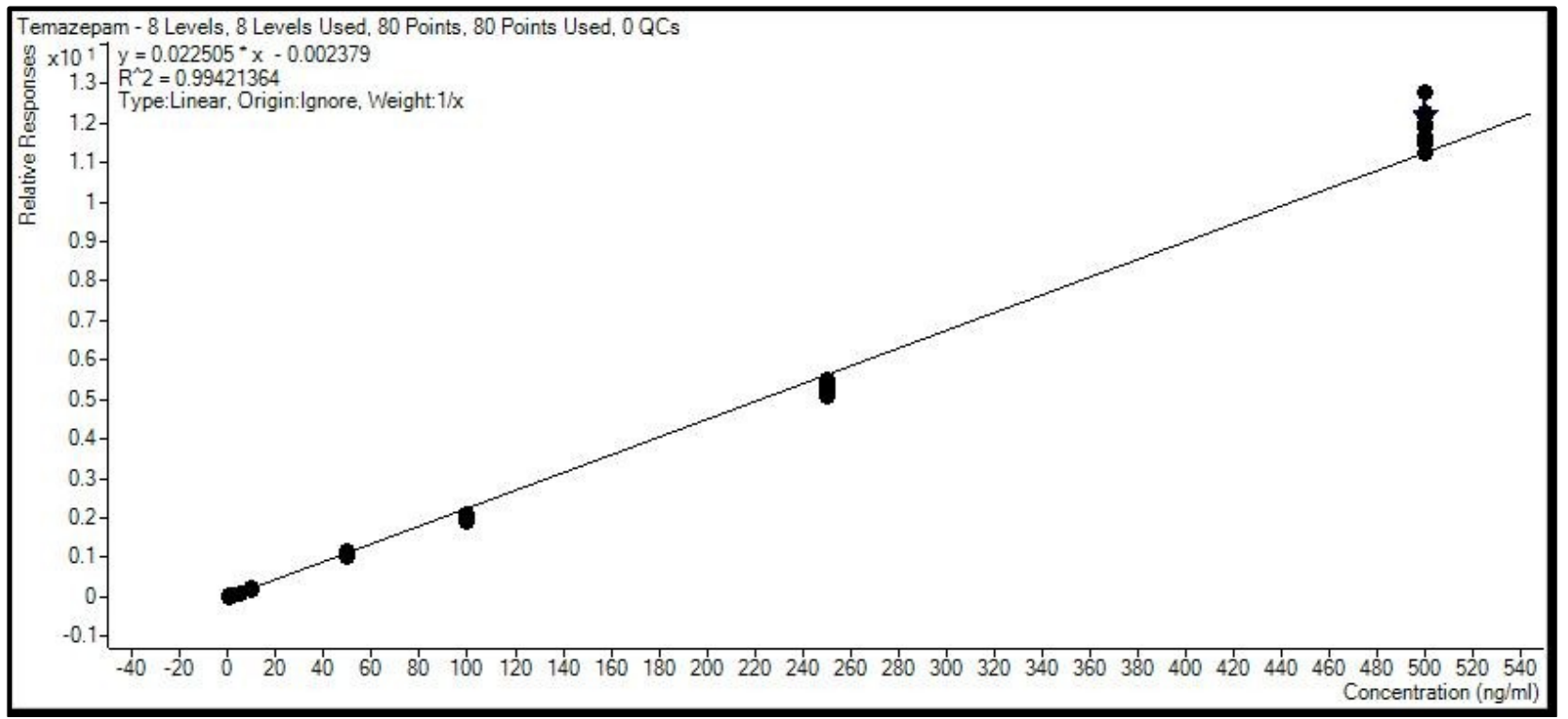




\section{Flunitrazepam}

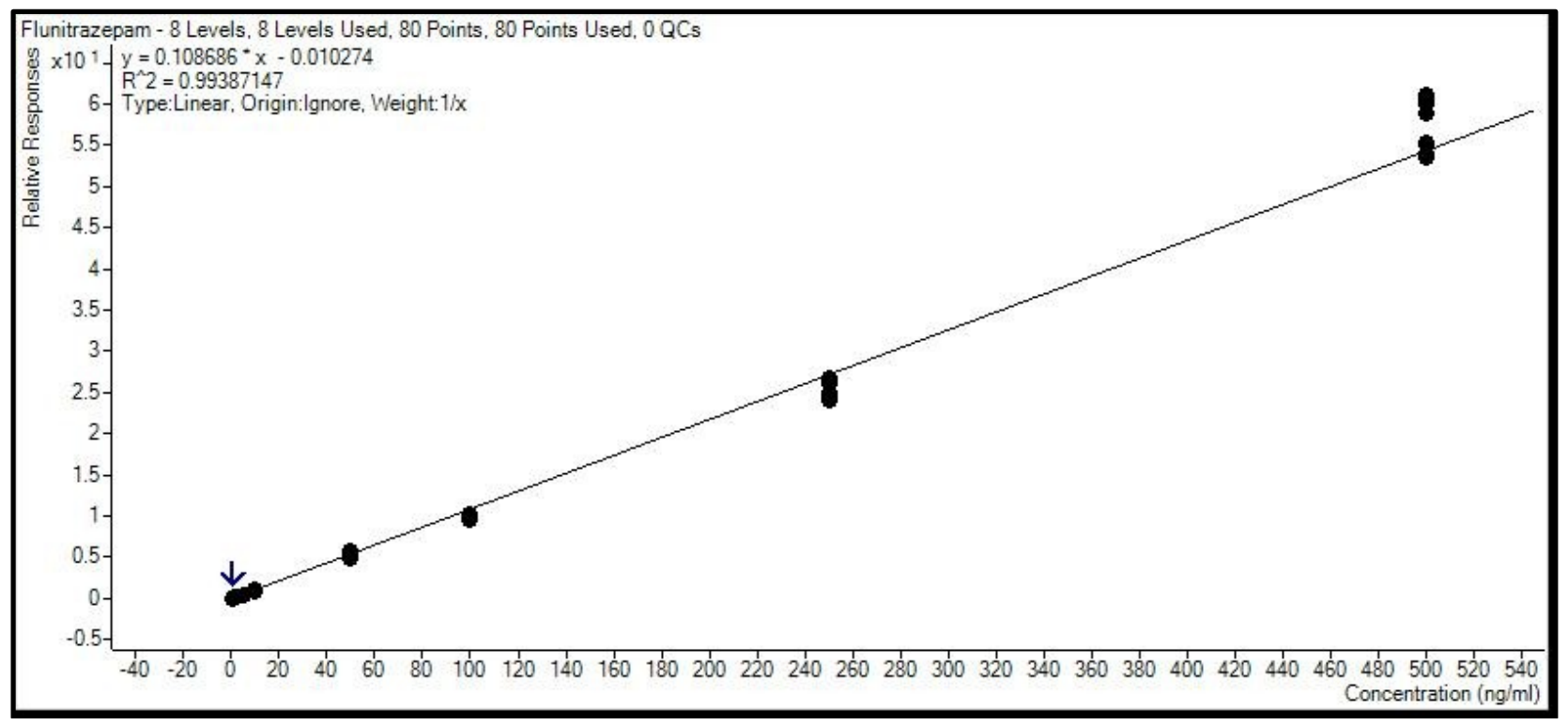

\section{7-Aminoflunitrazepam}

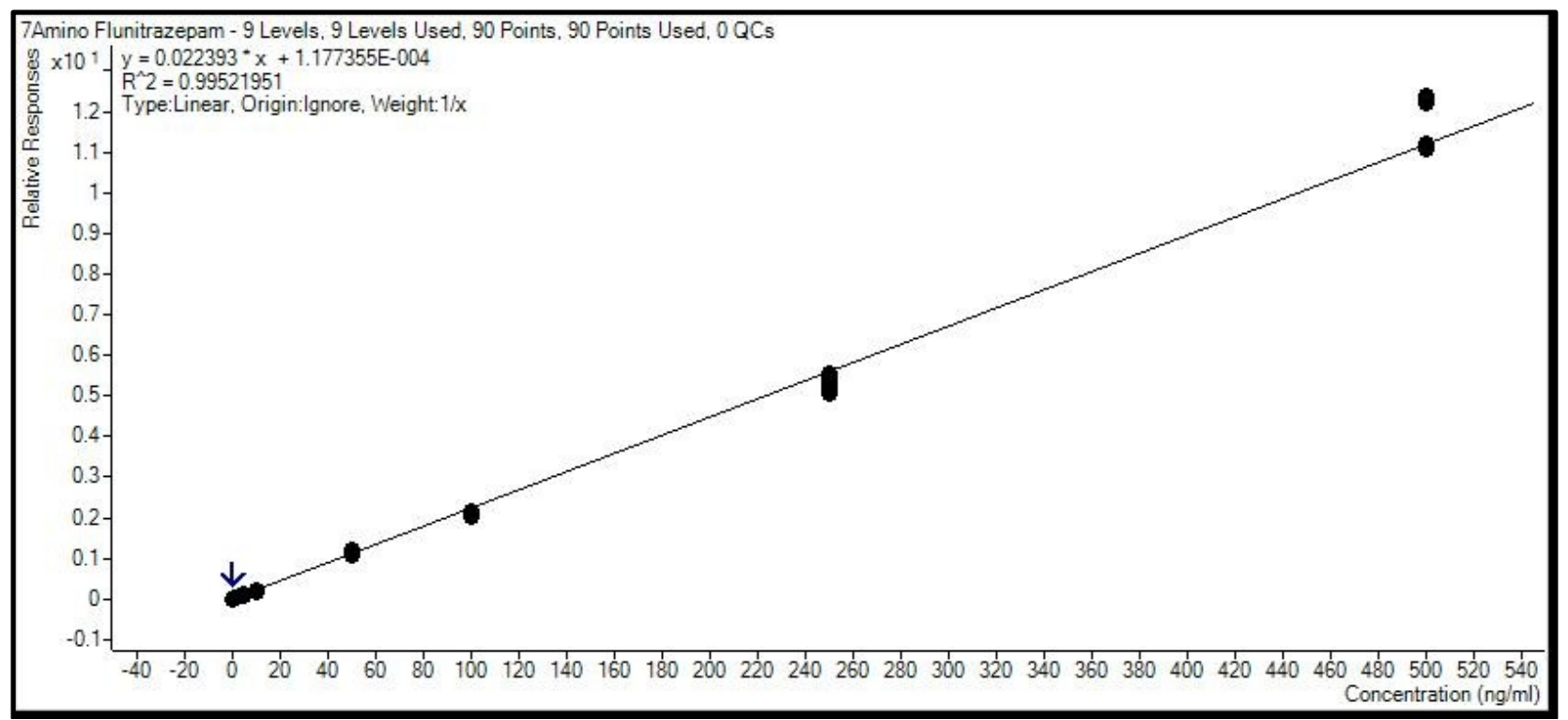




\section{N-Desmethylflunitrazepam}

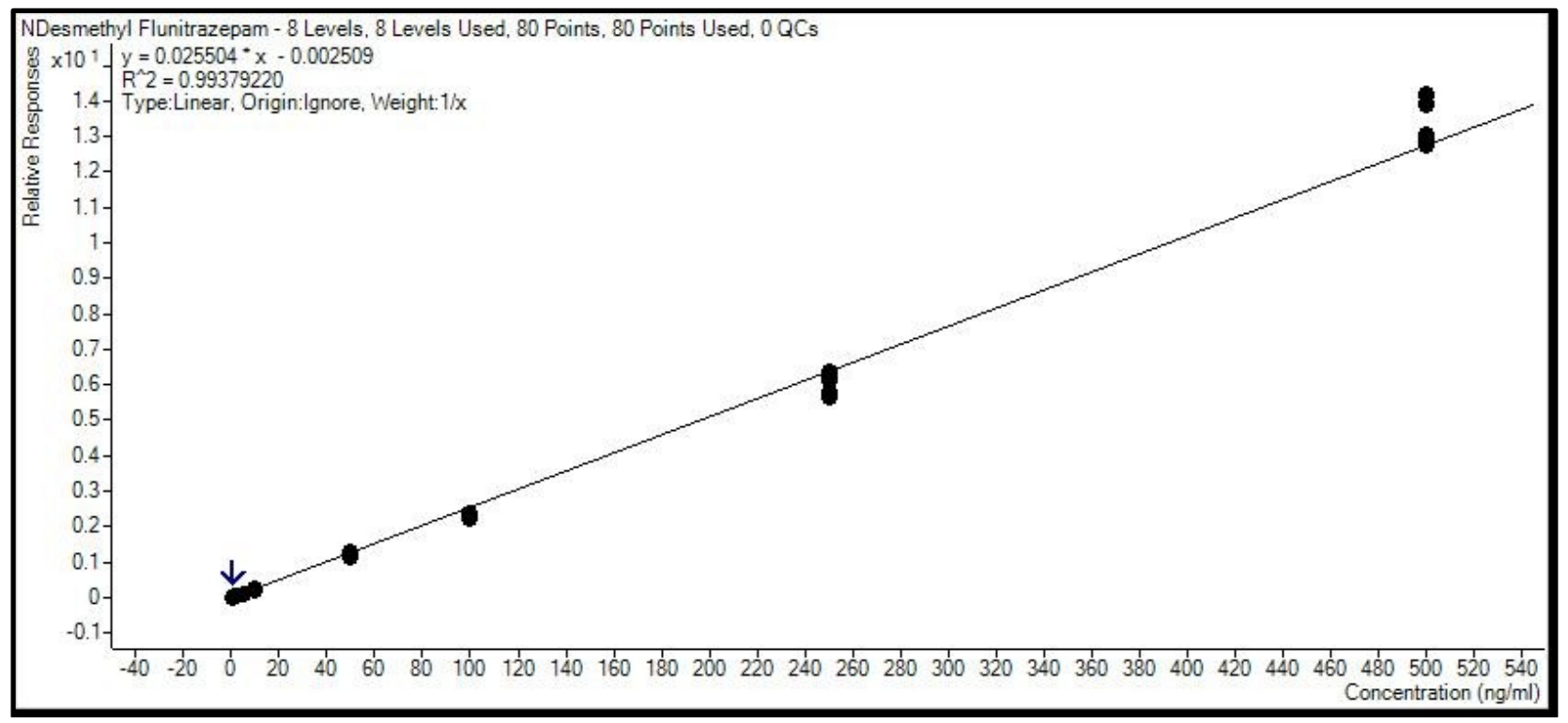




\section{Appendix C - Optimized Precursor \& Product lons for Diazepam,}

Flunitrazepam, and Metabolites

\section{Diazepam Optimized Precursor \& Product lons}

\begin{tabular}{|ccccc|}
\hline \multirow{3}{*}{ Compound } & Fragmentor & CE $(\mathbf{V})$ & Precursor $(\boldsymbol{m} / \mathbf{z})$ & Product $(\boldsymbol{m} / \mathbf{z})$ \\
\hline \multirow{3}{*}{ DZ } & 107 & 29 & 285.1 & 193.0 \\
& 107 & 25 & 285.1 & 154.0 \\
& 107 & 25 & 285.1 & 222.1 \\
& 107 & 45 & 285.1 & 91.0 \\
\hline \multirow{3}{*}{ NDZ } & 112 & 25 & 271.1 & 140.0 \\
& 112 & 25 & 271.1 & 208.0 \\
& 112 & 25 & 271.1 & 165.0 \\
& 112 & 37 & 271.1 & 91.0 \\
\hline \multirow{3}{*}{ OX } & 112 & 17 & 287.1 & 241.0 \\
& 112 & 9 & 287.1 & 268.9 \\
& 112 & 37 & 287.1 & 104.0 \\
& 112 & 36 & 287.1 & 162.9 \\
\hline \multirow{2}{*}{ TZ } & 107 & 17 & 301.1 & 255.0 \\
& 107 & 5 & 301.1 & 282.9 \\
& 107 & 41 & 301.1 & 177.0 \\
& 107 & 33 & 301.1 & 193.0 \\
\hline
\end{tabular}

Flunitrazepam Optimized Precursor \& Product Ions

\begin{tabular}{|ccccc|}
\hline \multirow{3}{*}{ Compound } & Fragmentor & CE (V) & Precursor $(\boldsymbol{m} / \mathbf{z})$ & Product $(\boldsymbol{m} / \mathbf{z})$ \\
\hline \multirow{3}{*}{ FLU } & 135 & 21 & 314.1 & 268.1 \\
& 135 & 33 & 314.1 & 239.1 \\
& 135 & 49 & 314.1 & 183.1 \\
& 135 & 33 & 314.1 & 211.0 \\
\hline \multirow{3}{*}{ 7AM } & 140 & 25 & 284.1 & 135.0 \\
& 140 & 17 & 284.1 & 227.1 \\
& 140 & 29 & 284.1 & 226.2 \\
\multirow{3}{*}{ NDES } & 140 & 29 & 284.1 & 240.1 \\
& 125 & 21 & 300.1 & 253.9 \\
& 125 & 37 & 300.1 & 197.9 \\
& 125 & 33 & 300.1 & 225.1 \\
& 125 & 29 & 300.1 & 205.9 \\
\hline
\end{tabular}




\section{Appendix D - Calibration Curves for Cocaine, Fentanyl, and Metabolites in Buffer}

\section{Cocaine}

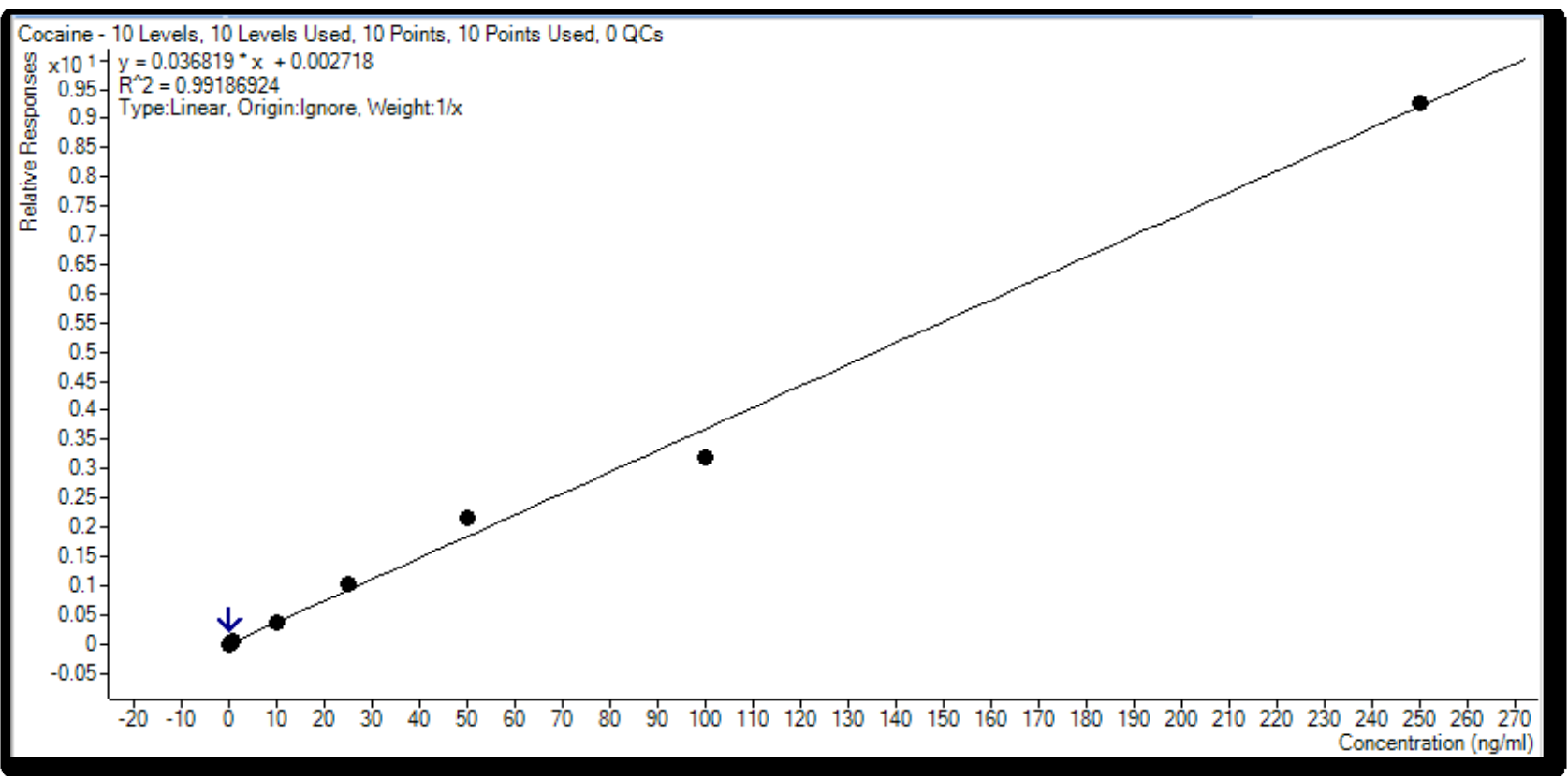

\section{Anhydroecgonine Methyl Ester}

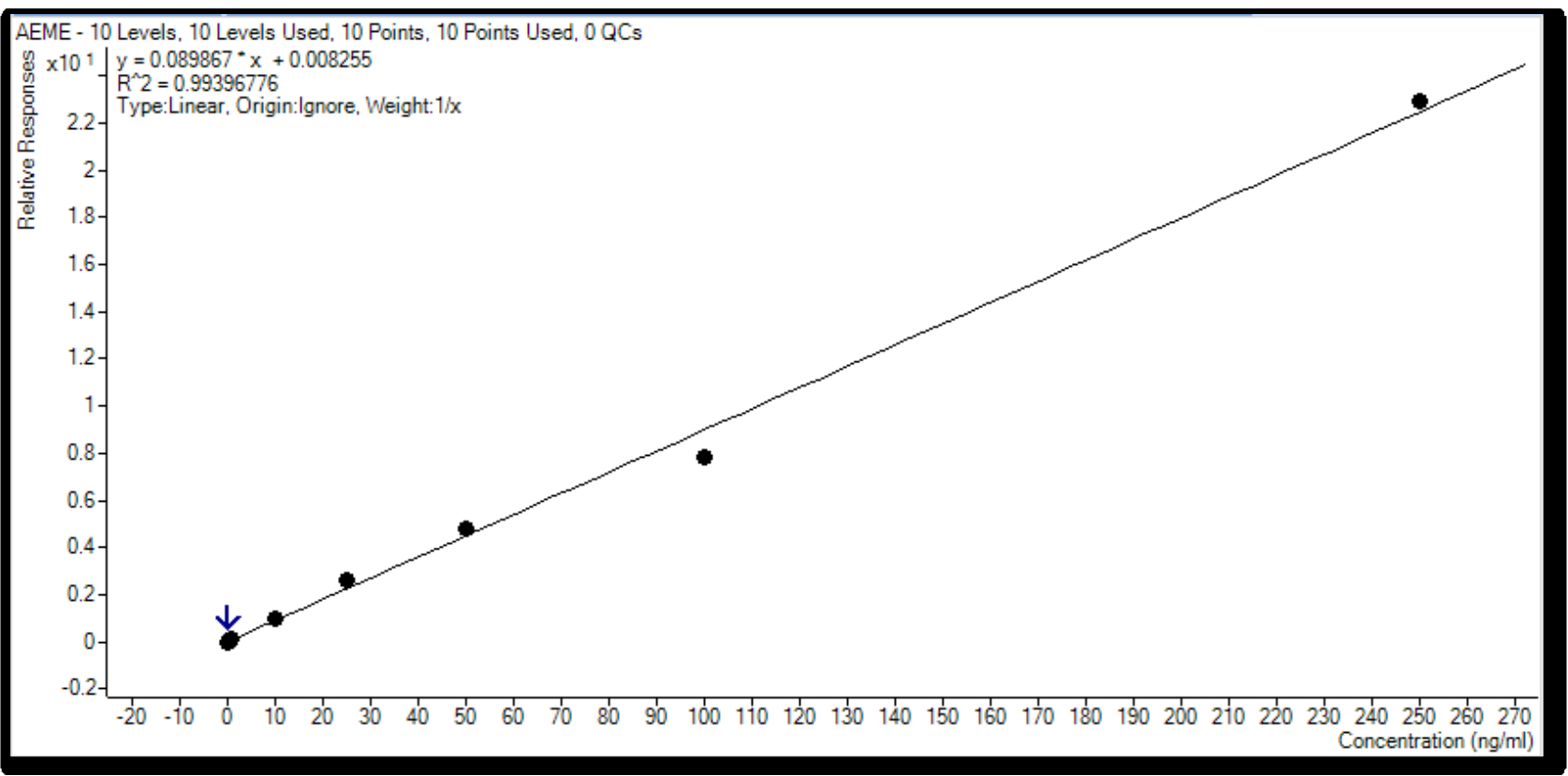




\section{Benzoylecgonine}

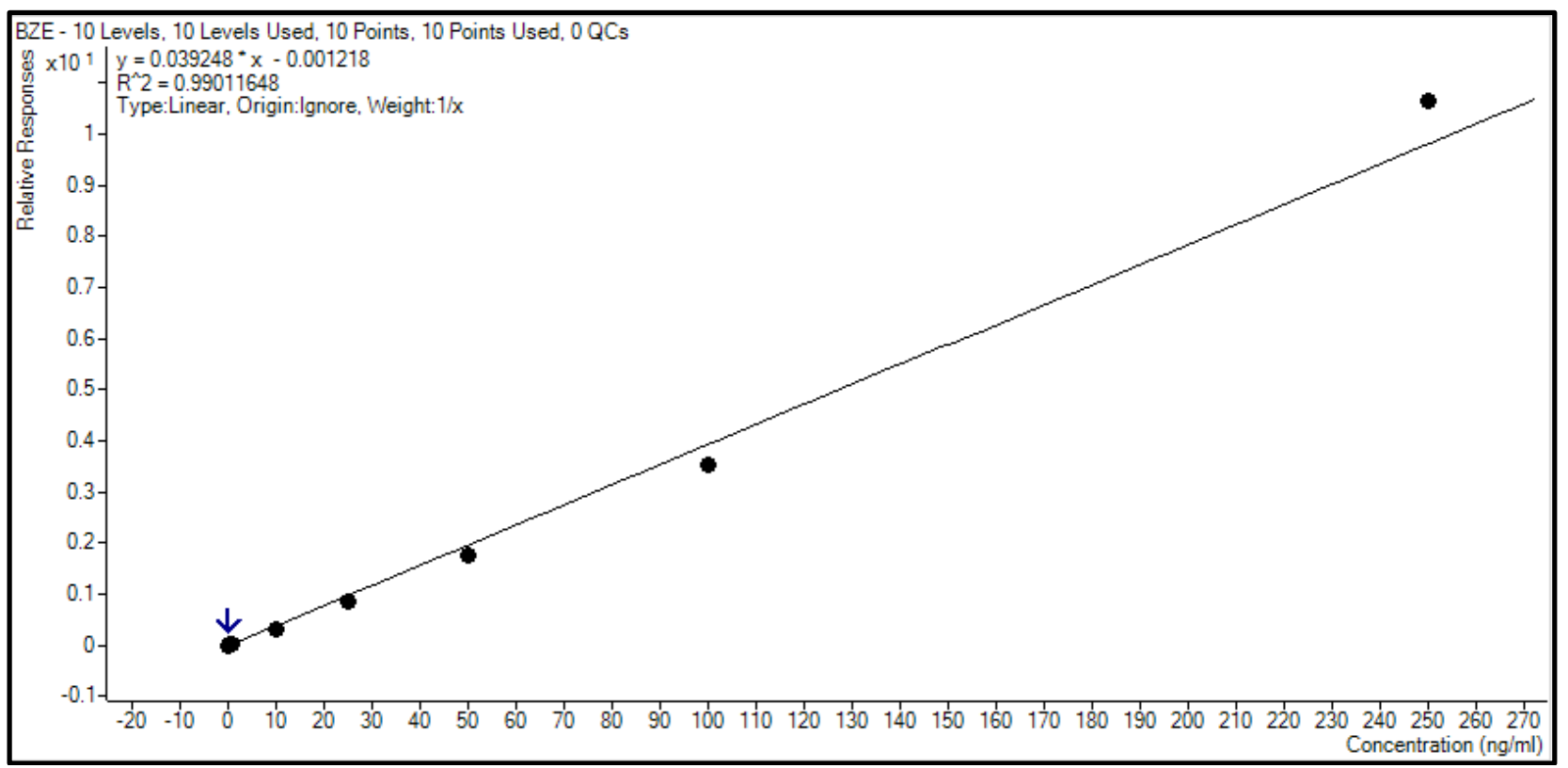

\section{Cocaethylene}

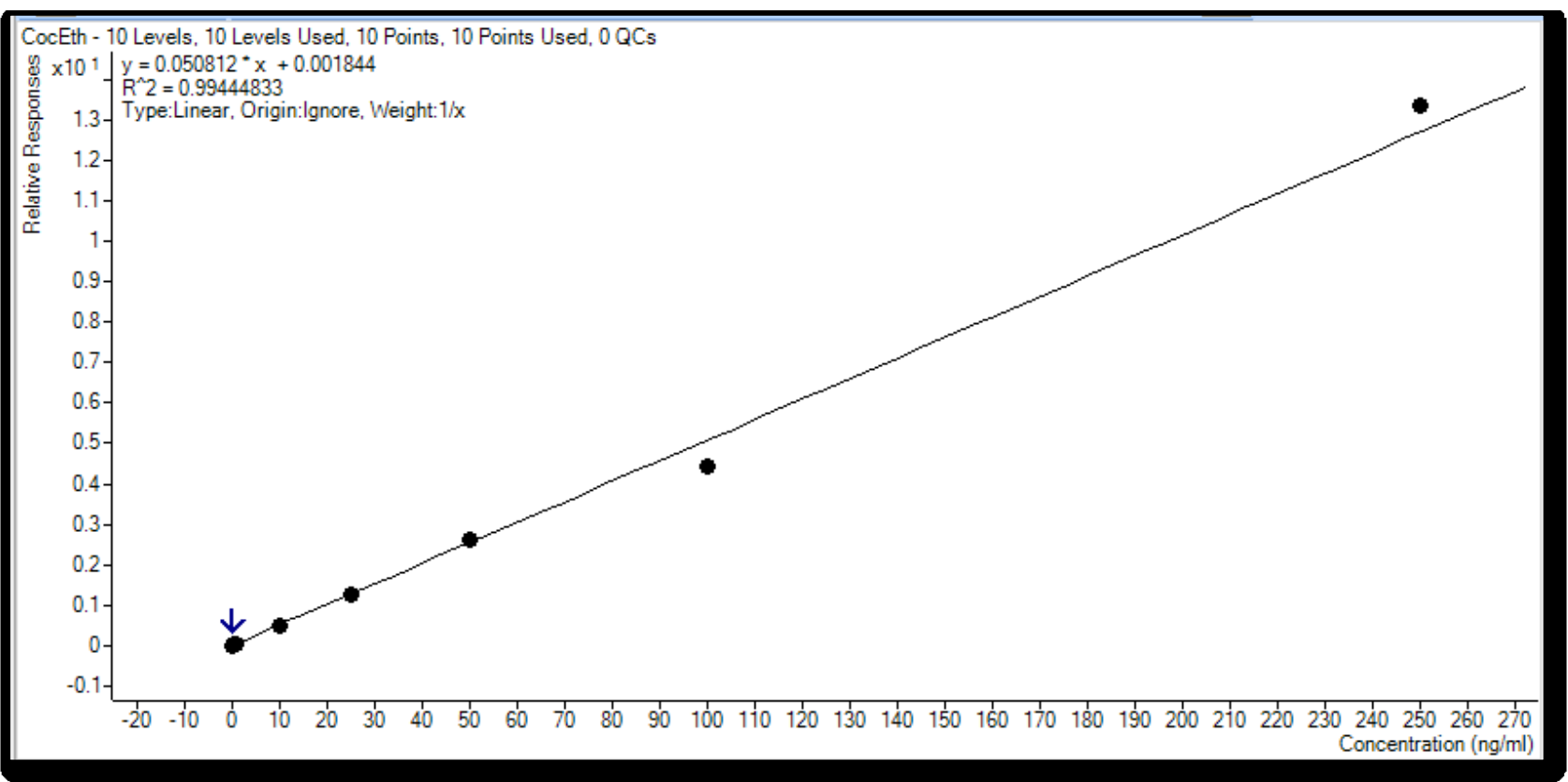




\section{Ecgonine Methyl Ester}

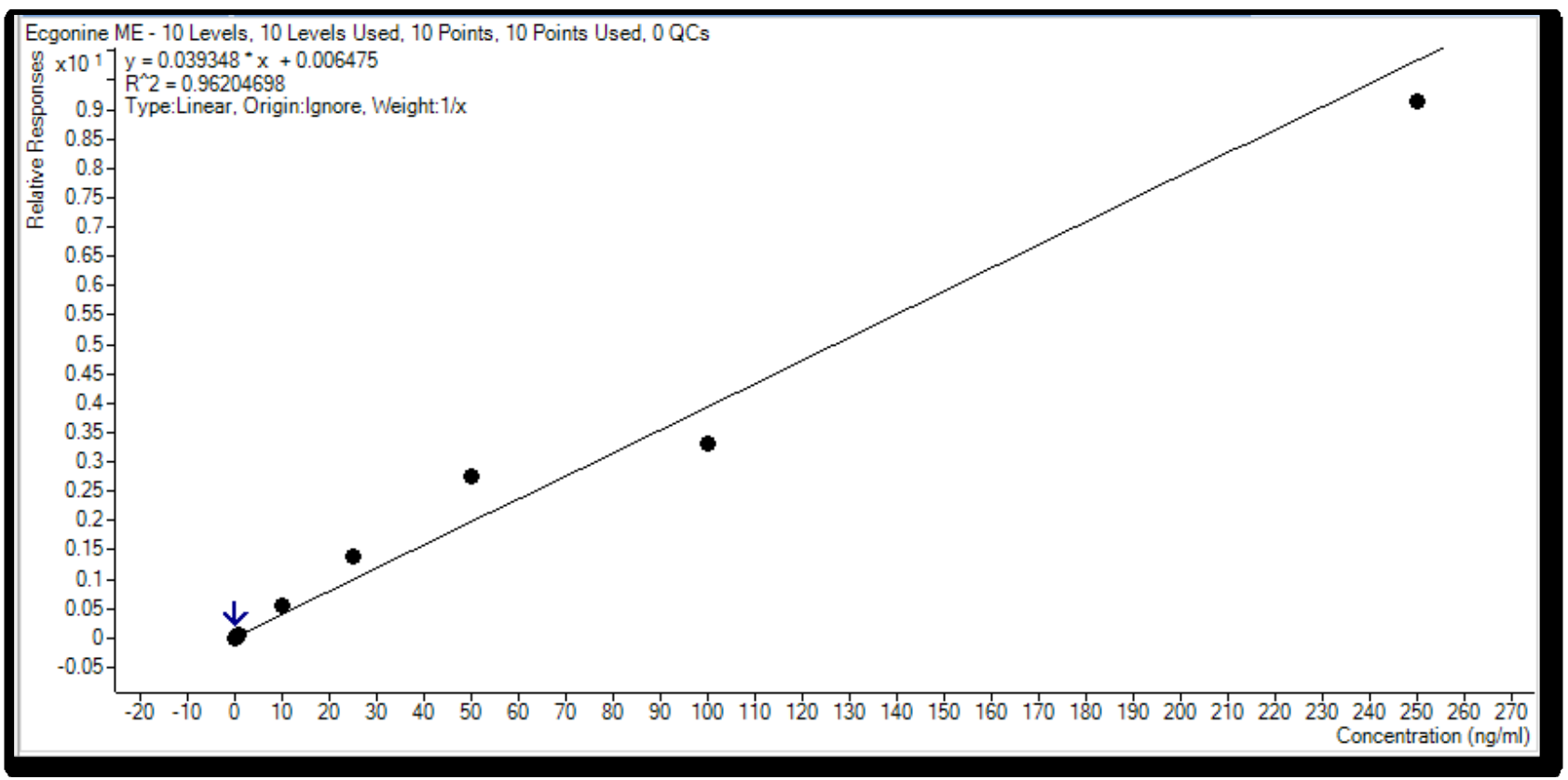

\section{m-Hydroxycocaine}

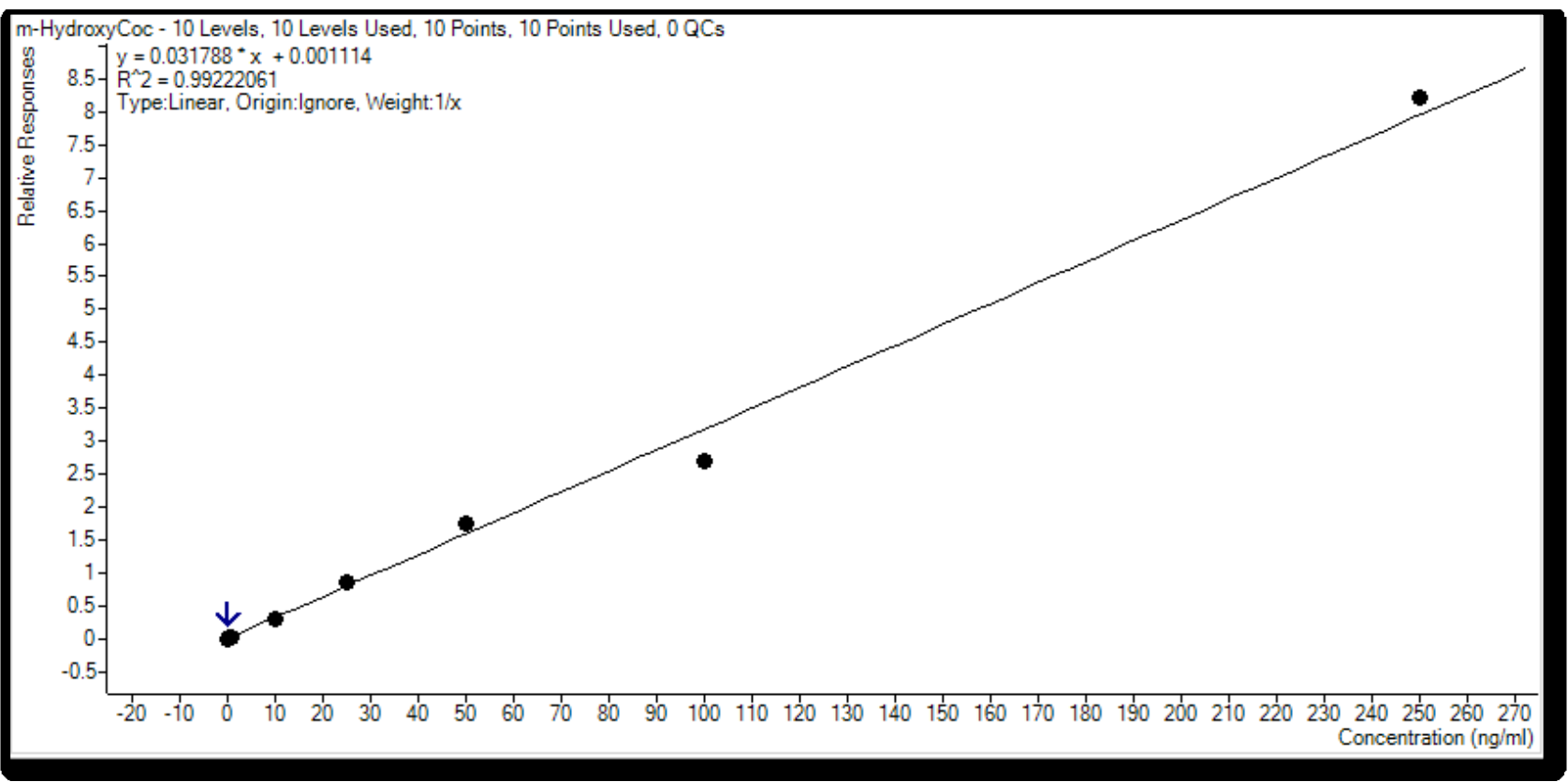




\section{Norcocaine}

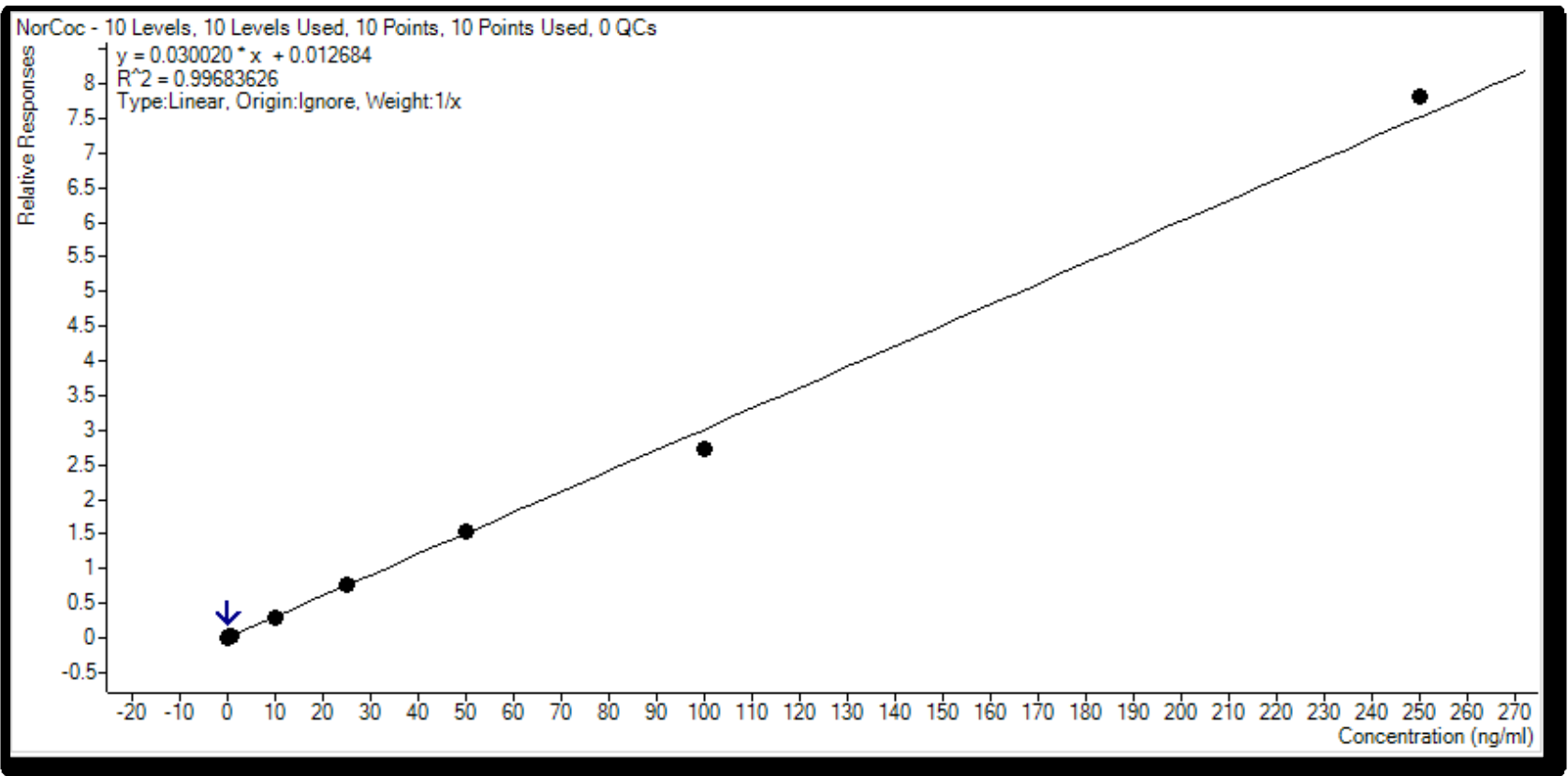

\section{Fentanyl}

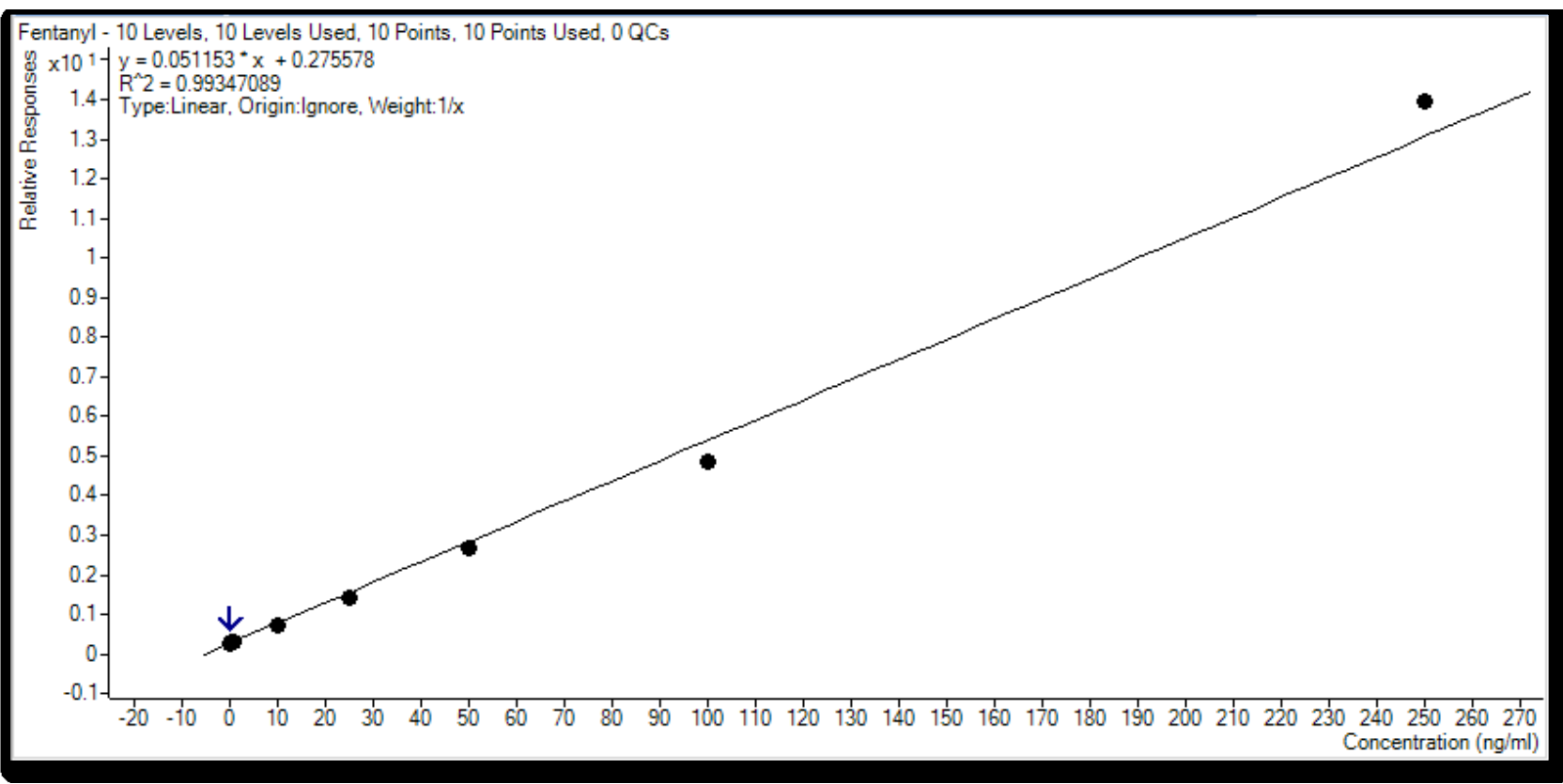




\section{Norfentanyl}

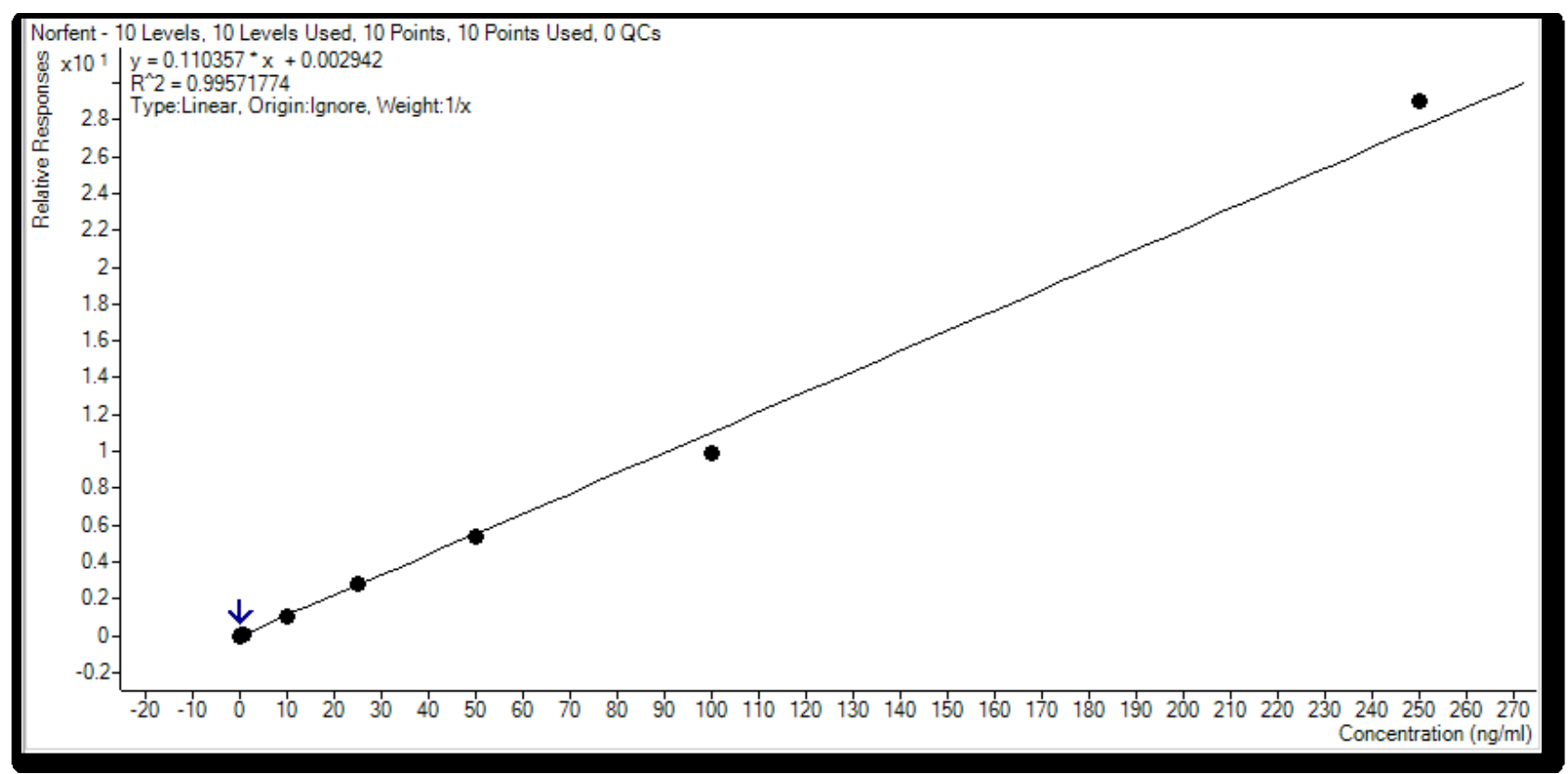

\section{Despropionylfentanyl}

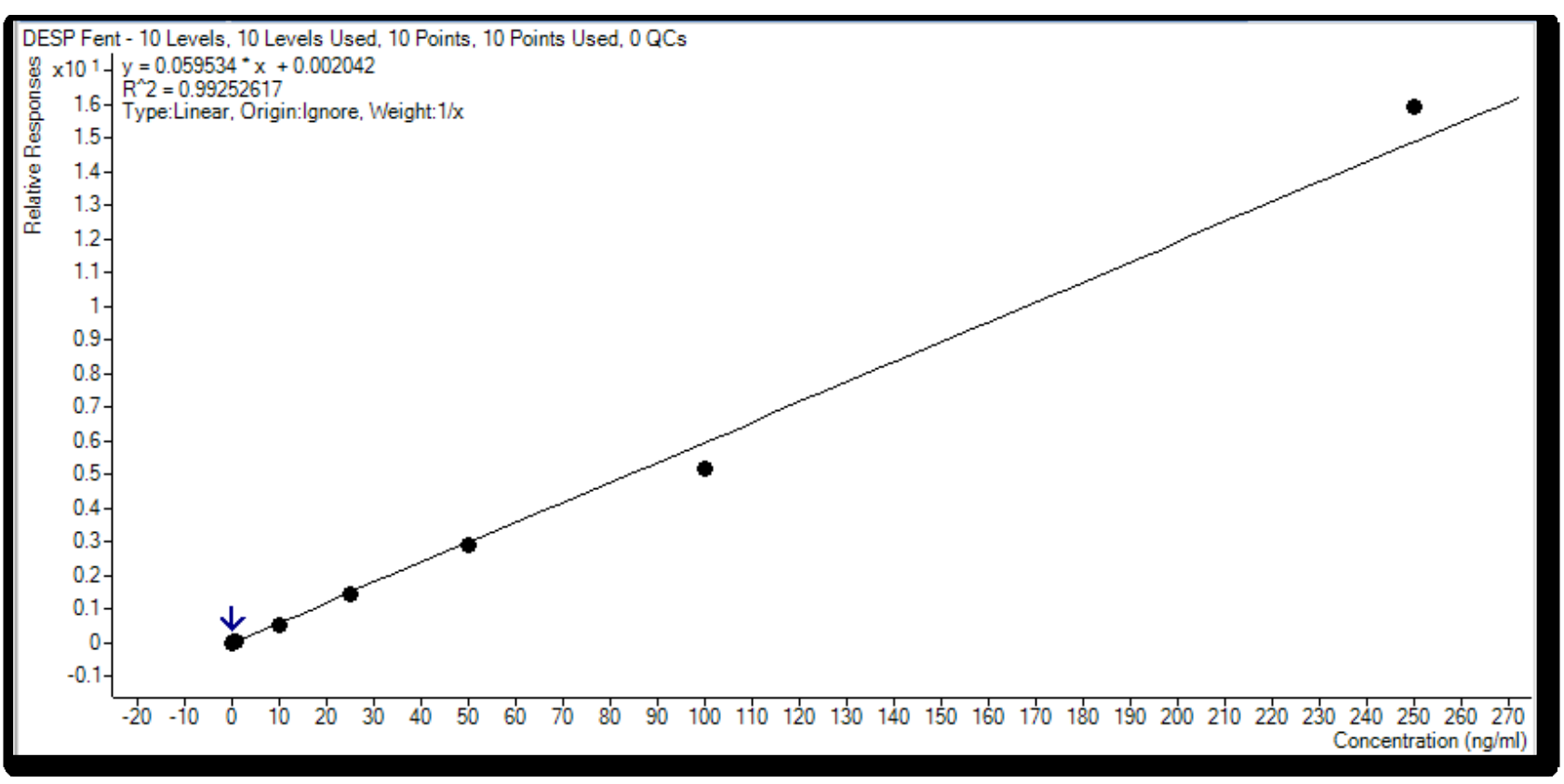




\section{Appendix E - Optimized Precursor \& Product lons for Cocaine, Fentanyl, and Metabolites}

Cocaine Optimized Precursor \& Product lons

\begin{tabular}{|c|c|c|c|c|}
\hline Compound & Fragmentor & CE & Precursor $(m / z)$ & Product $(\mathrm{m} / \mathrm{z})$ \\
\hline \multirow{4}{*}{ COC } & 96 & 13 & 304.2 & 182.0 \\
\hline & 96 & 20 & 304.2 & 82.0 \\
\hline & 96 & 20 & 304.2 & 105.0 \\
\hline & 96 & 20 & 304.2 & 150.0 \\
\hline \multirow{4}{*}{ AEME } & 96 & 20 & 182.1 & 118.0 \\
\hline & 96 & 20 & 182.1 & 91.0 \\
\hline & 96 & 17 & 182.1 & 122.0 \\
\hline & 96 & 13 & 182.2 & 150.4 \\
\hline \multirow{4}{*}{ BZE } & 96 & 13 & 290.1 & 168.0 \\
\hline & 96 & 20 & 290.1 & 104.9 \\
\hline & 96 & 20 & 290.1 & 82.0 \\
\hline & 96 & 20 & 290.1 & 150.0 \\
\hline \multirow{4}{*}{ CE } & 96 & 13 & 318.2 & 196.0 \\
\hline & 96 & 20 & 318.2 & 82.0 \\
\hline & 96 & 20 & 318.2 & 150.0 \\
\hline & 96 & 20 & 318.2 & 108.0 \\
\hline \multirow{4}{*}{ EME } & 96 & 13 & 200.1 & 182.0 \\
\hline & 96 & 20 & 200.1 & 82.0 \\
\hline & 96 & 20 & 200.1 & 150.0 \\
\hline & 96 & 20 & 200.1 & 108.0 \\
\hline \multirow{4}{*}{$\mathrm{mHC}$} & 96 & 13 & 320.2 & 182.0 \\
\hline & 96 & 20 & 320.2 & 82.0 \\
\hline & 96 & 20 & 320.2 & 150.0 \\
\hline & 96 & 20 & 320.2 & 120.9 \\
\hline \multirow{4}{*}{ NC } & 96 & 9 & 290.1 & 168.0 \\
\hline & 96 & 17 & 290.1 & 136.0 \\
\hline & 96 & 20 & 290.1 & 108.0 \\
\hline & 96 & 20 & 290.1 & 68.0 \\
\hline
\end{tabular}


Fentanyl Optimized Precursor \& Product lons

\begin{tabular}{|ccccc|}
\hline \multirow{2}{*}{ Compound } & Fragmentor & CE & Precursor $(\boldsymbol{m} / \mathbf{z})$ & Product $(\boldsymbol{m} / \mathbf{z})$ \\
\hline \multirow{3}{*}{ FENT } & 130 & 19 & 337.2 & 188.0 \\
& 130 & 43 & 337.2 & 105.0 \\
& 130 & 50 & 337.2 & 79.0 \\
& 130 & 31 & 337.2 & 132.0 \\
\multirow{3}{*}{ NFENT } & 100 & 15 & 233.17 & 84.0 \\
& 100 & 43 & 233.17 & 55.1 \\
& 100 & 31 & 233.17 & 93.9 \\
& 100 & 50 & 233.17 & 128.6 \\
\hline \multirow{3}{*}{ DESP } & 100 & 31 & 281.2 & 105.0 \\
& 100 & 15 & 281.2 & 188.0 \\
& 100 & 50 & 281.2 & 79.0 \\
& 100 & 50 & 281.2 & 103.0 \\
\hline
\end{tabular}




\section{Appendix F - Calibration Curves for Cocaine, Fentanyl, and Metabolites in Media}

\section{Cocaine}

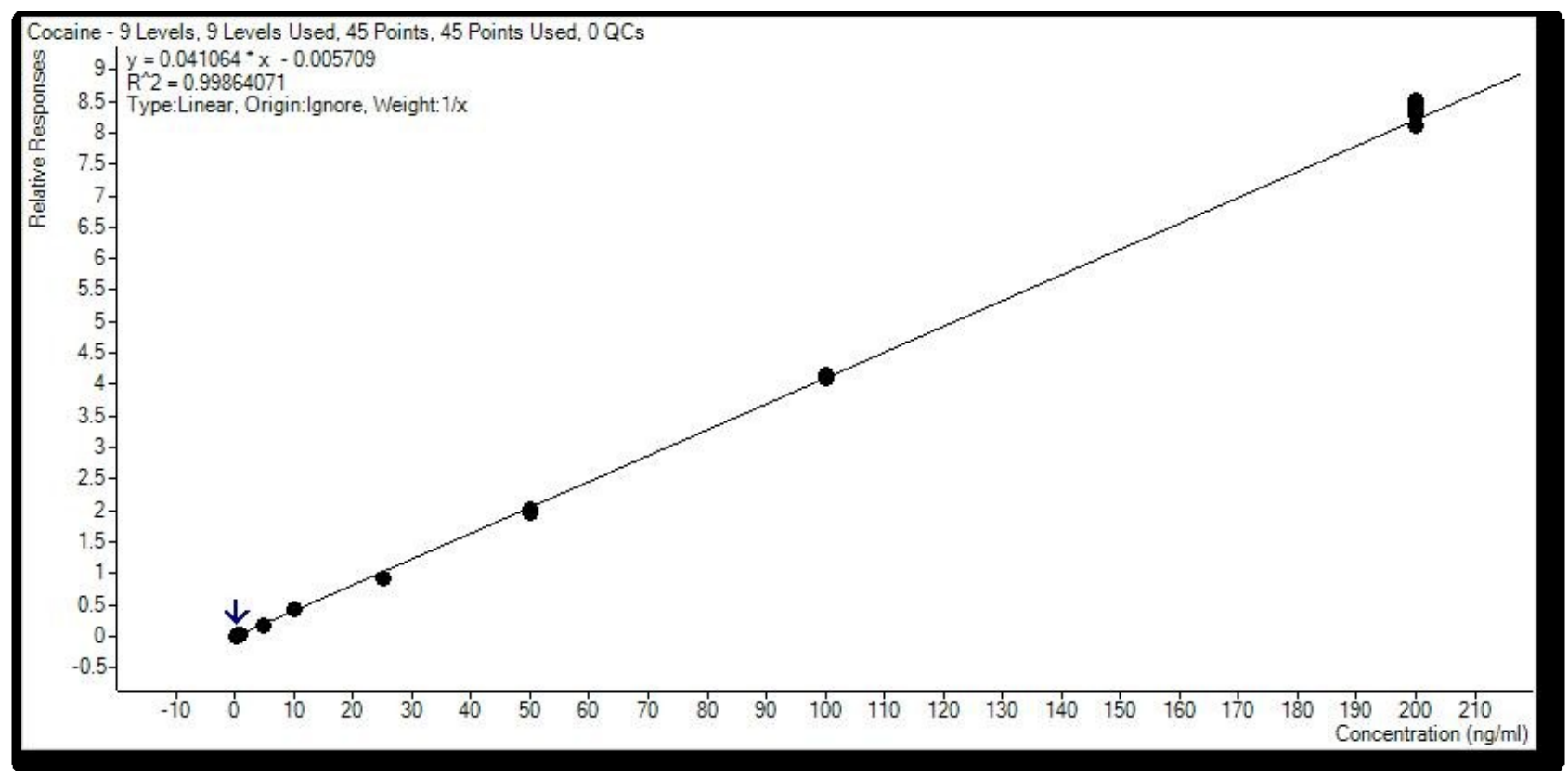

\section{Anhydroecgonine Methyl Ester}

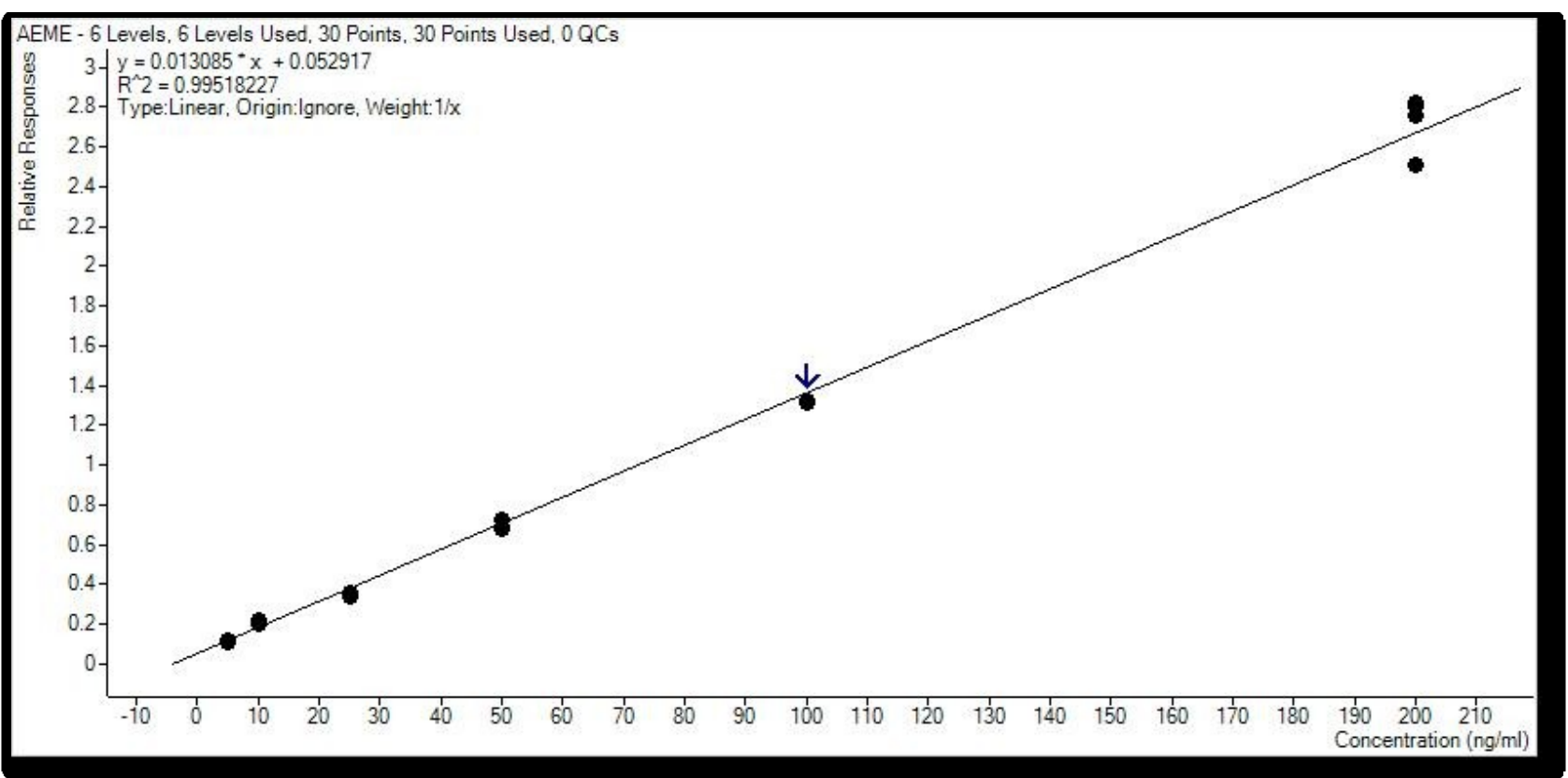




\section{Benzoylecgonine}

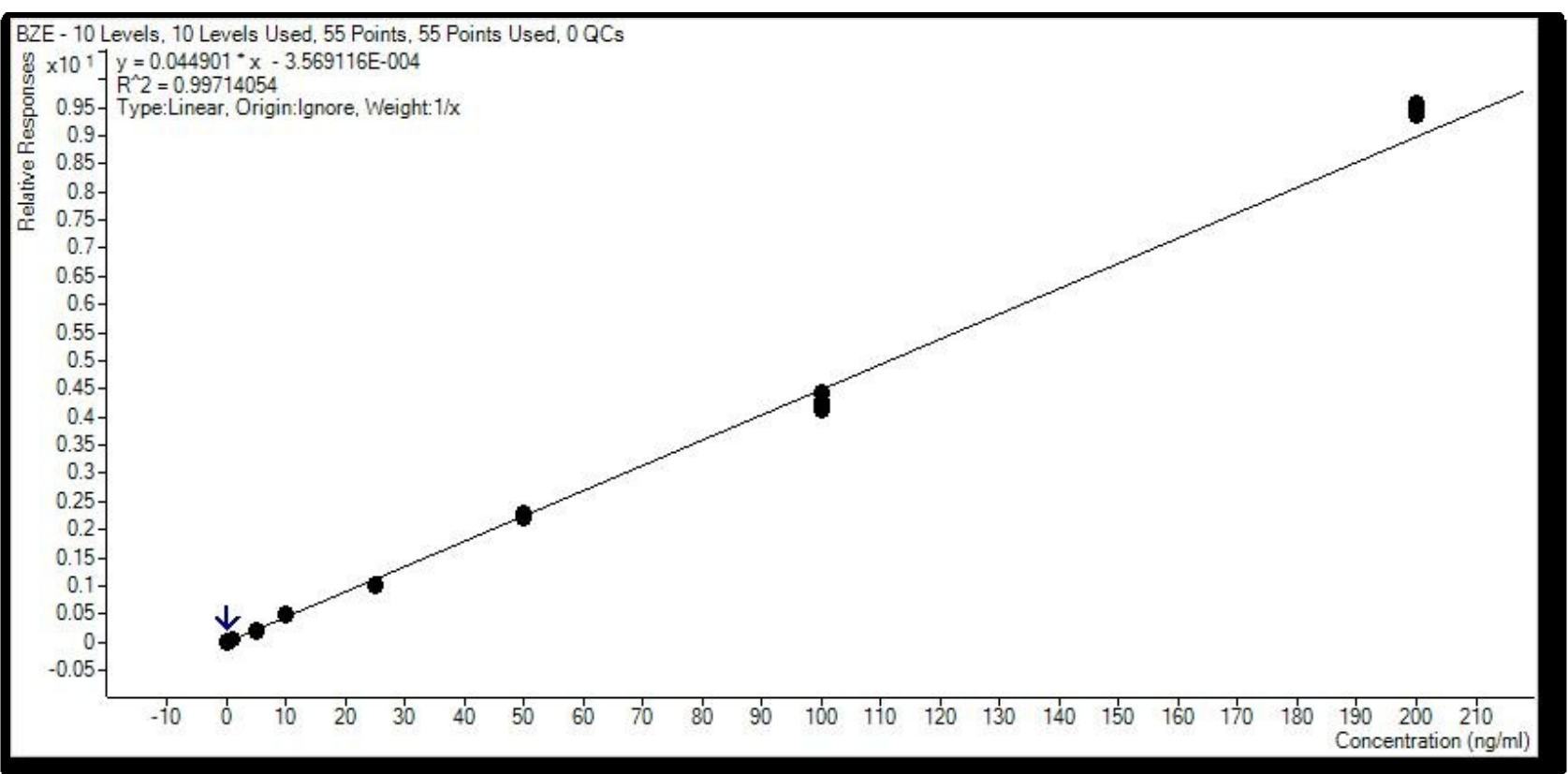

\section{Cocaethylene}

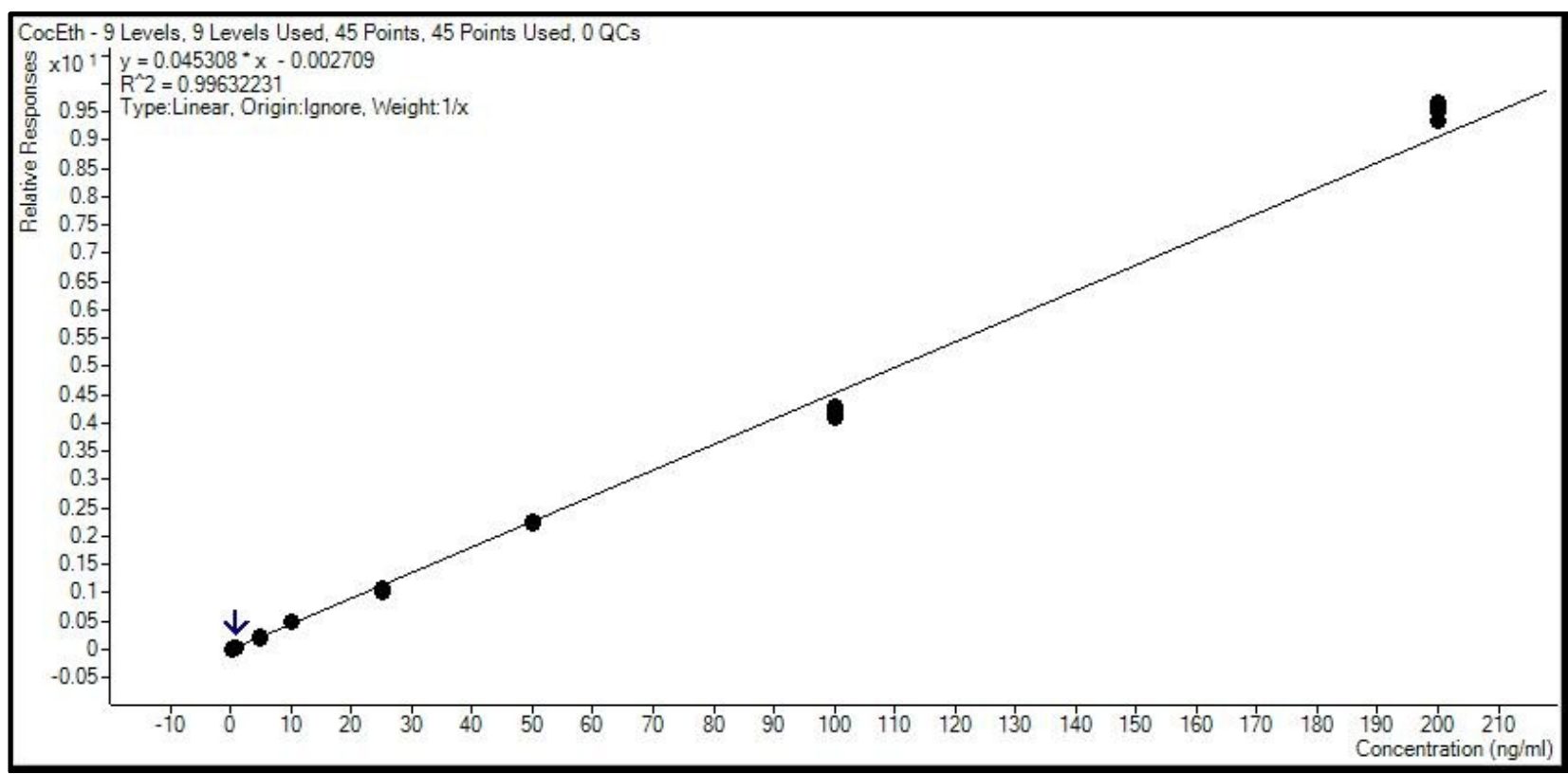




\section{Ecgonine Methyl Ester}

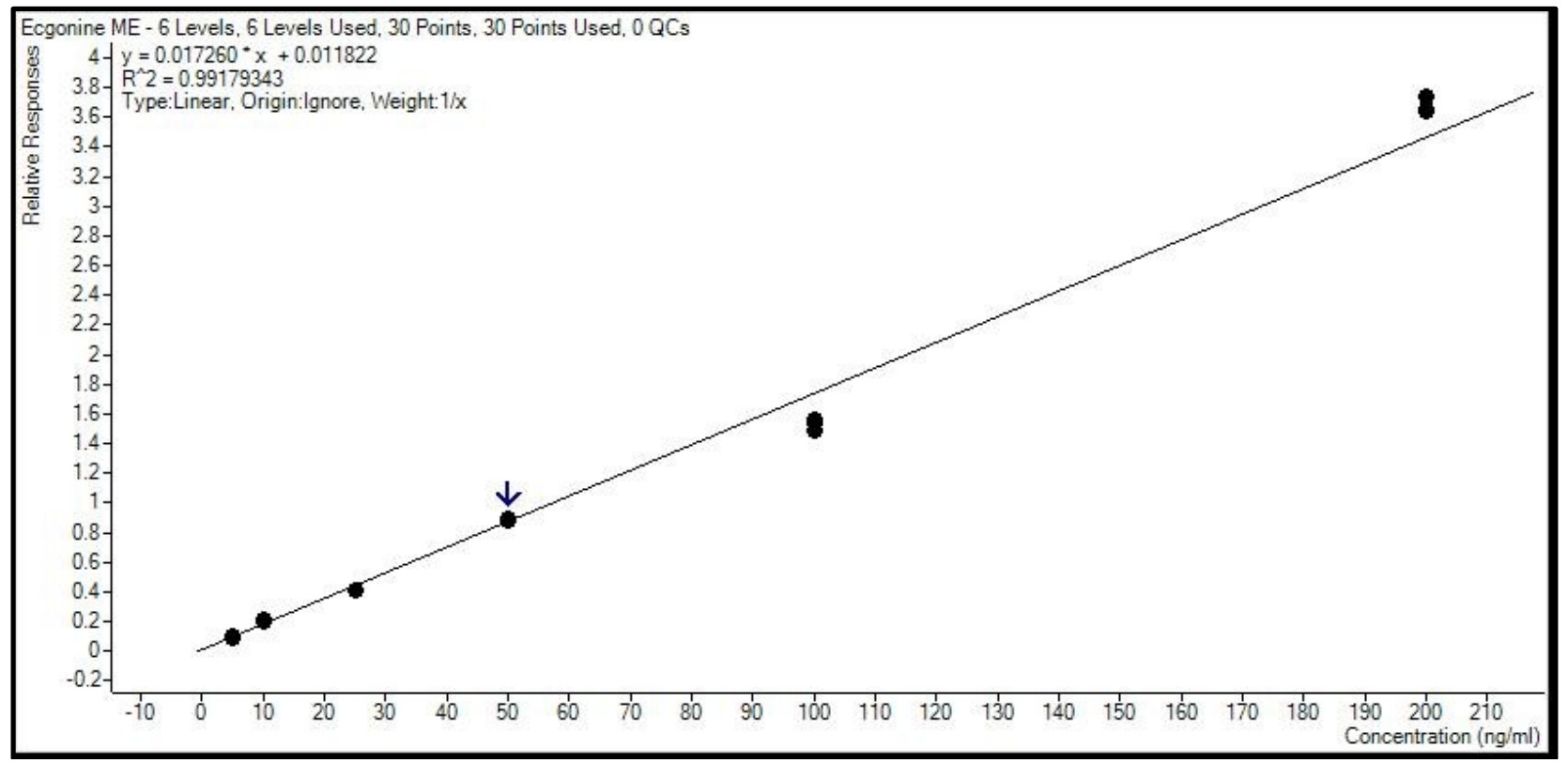

\section{m-Hydroxycocaine}

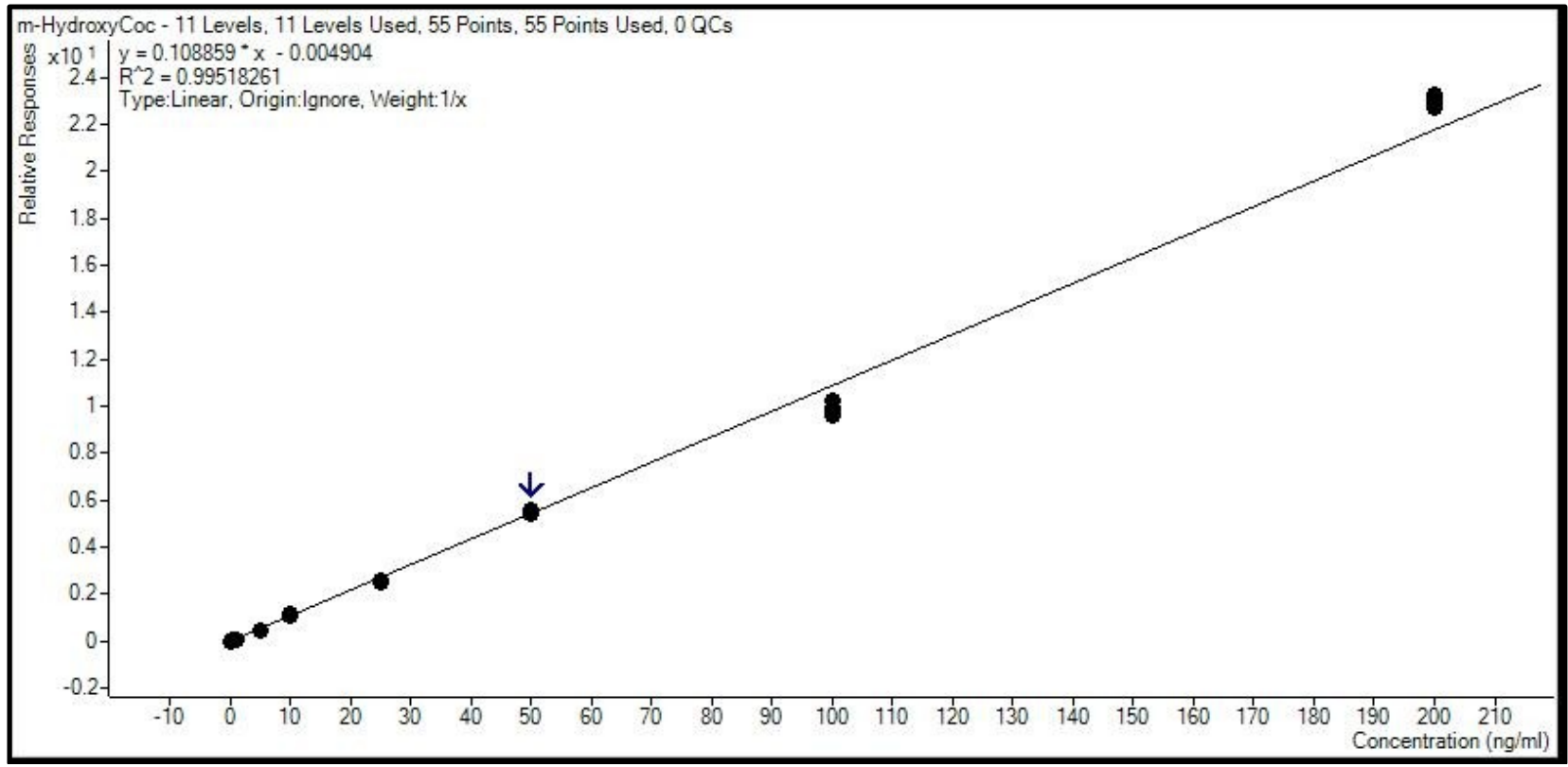




\section{Norcocaine}

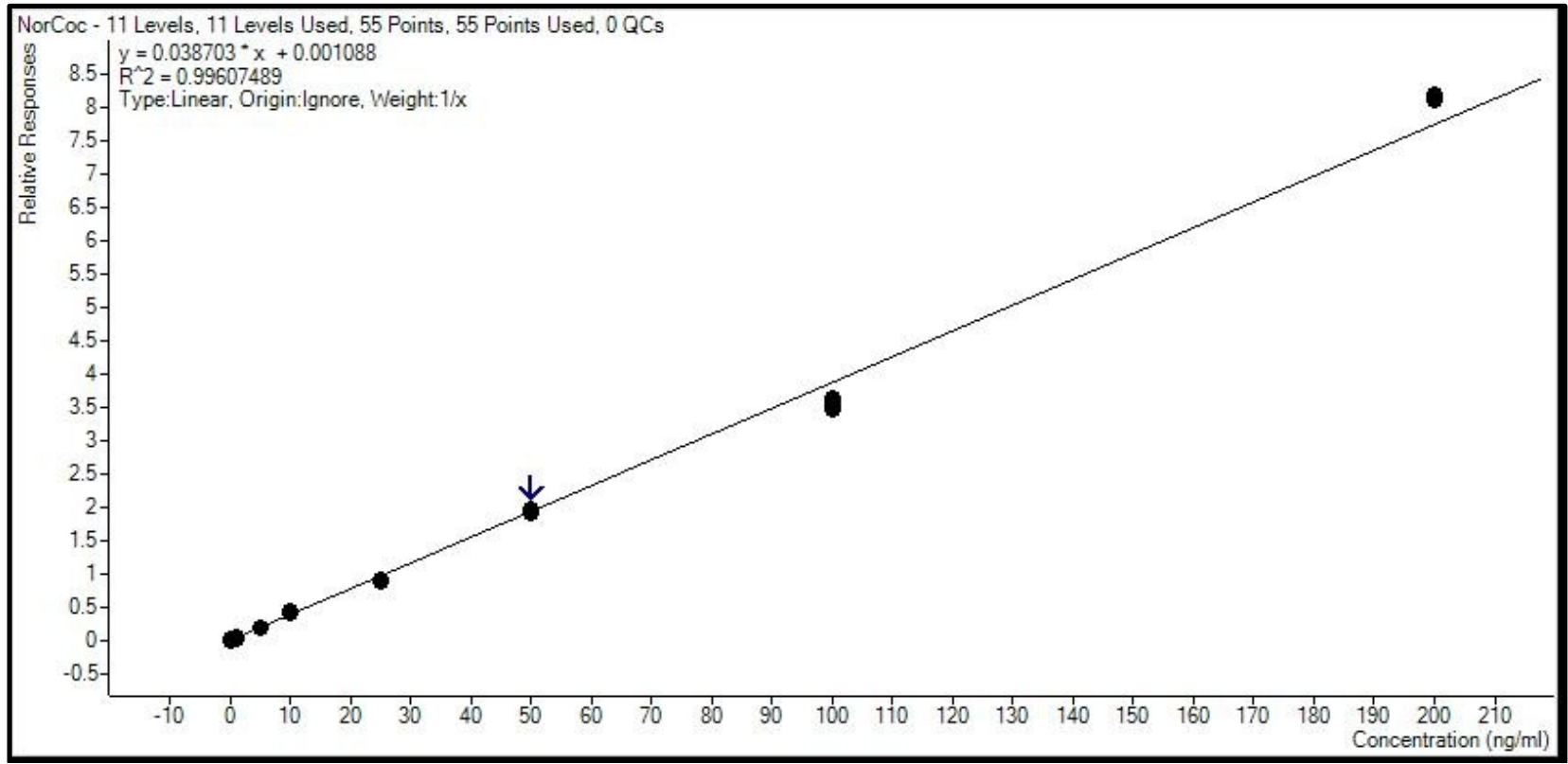

\section{Fentanyl}

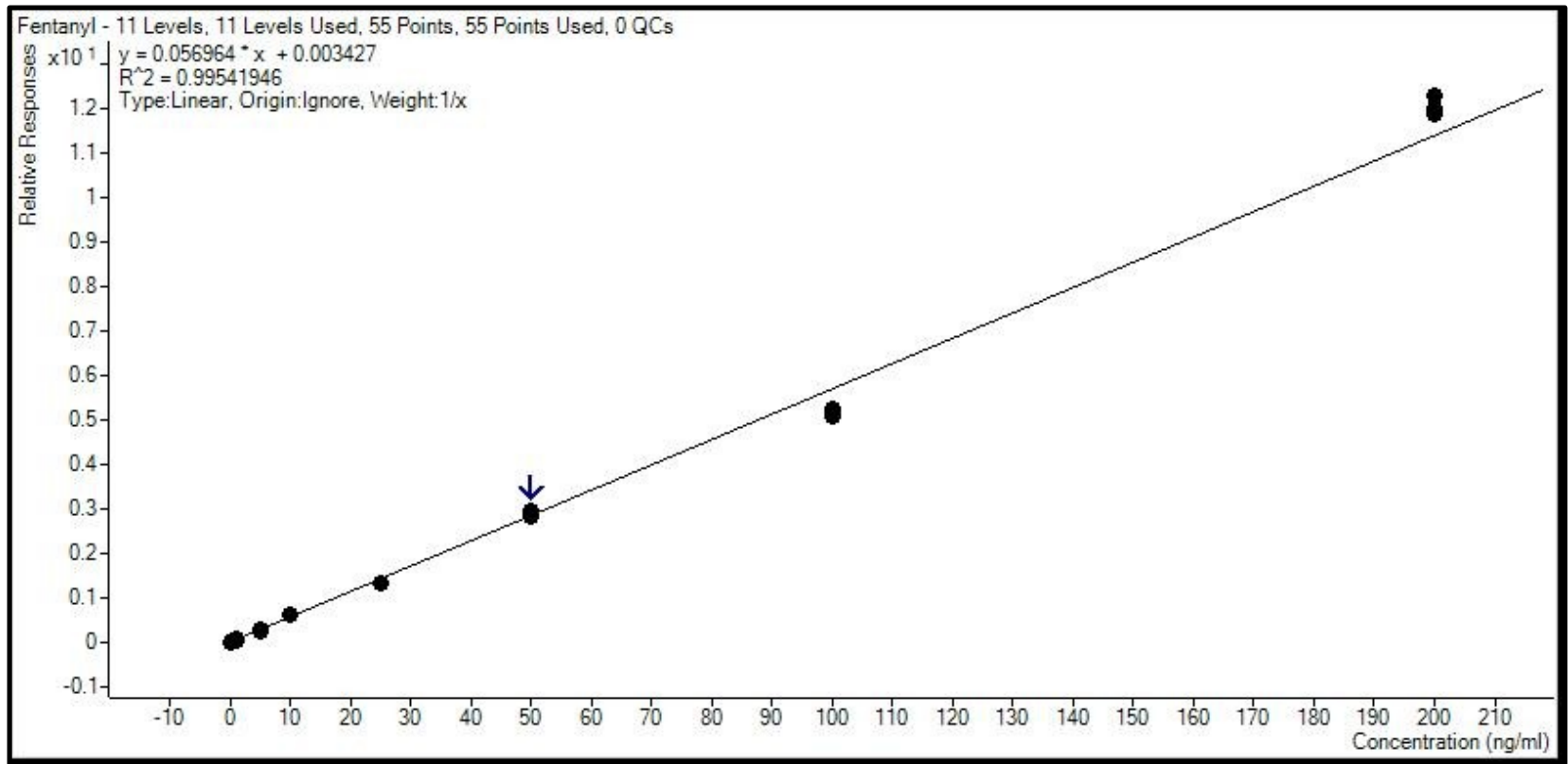




\section{Norfentanyl}

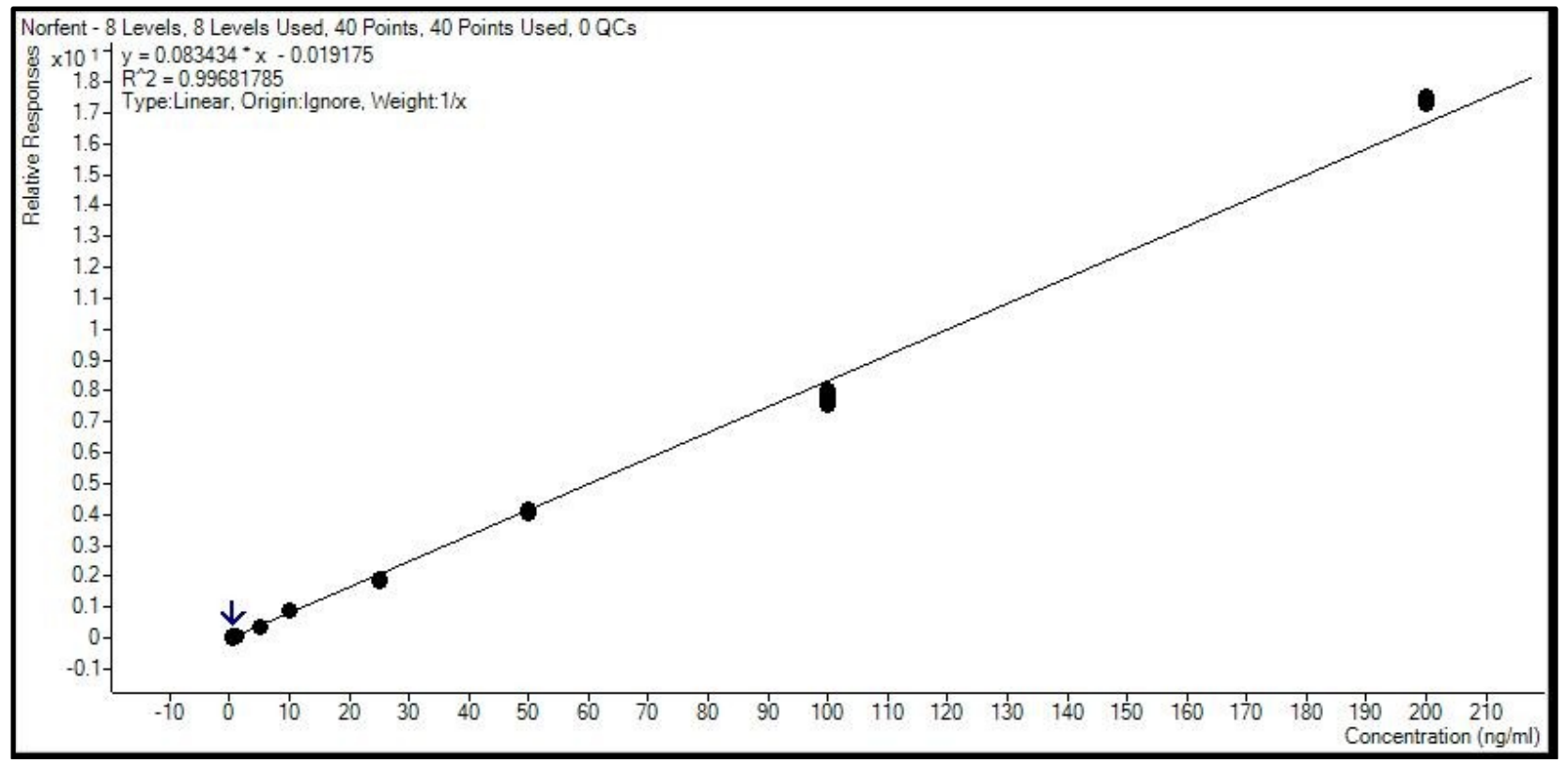

\section{Despropionylfentanyl}

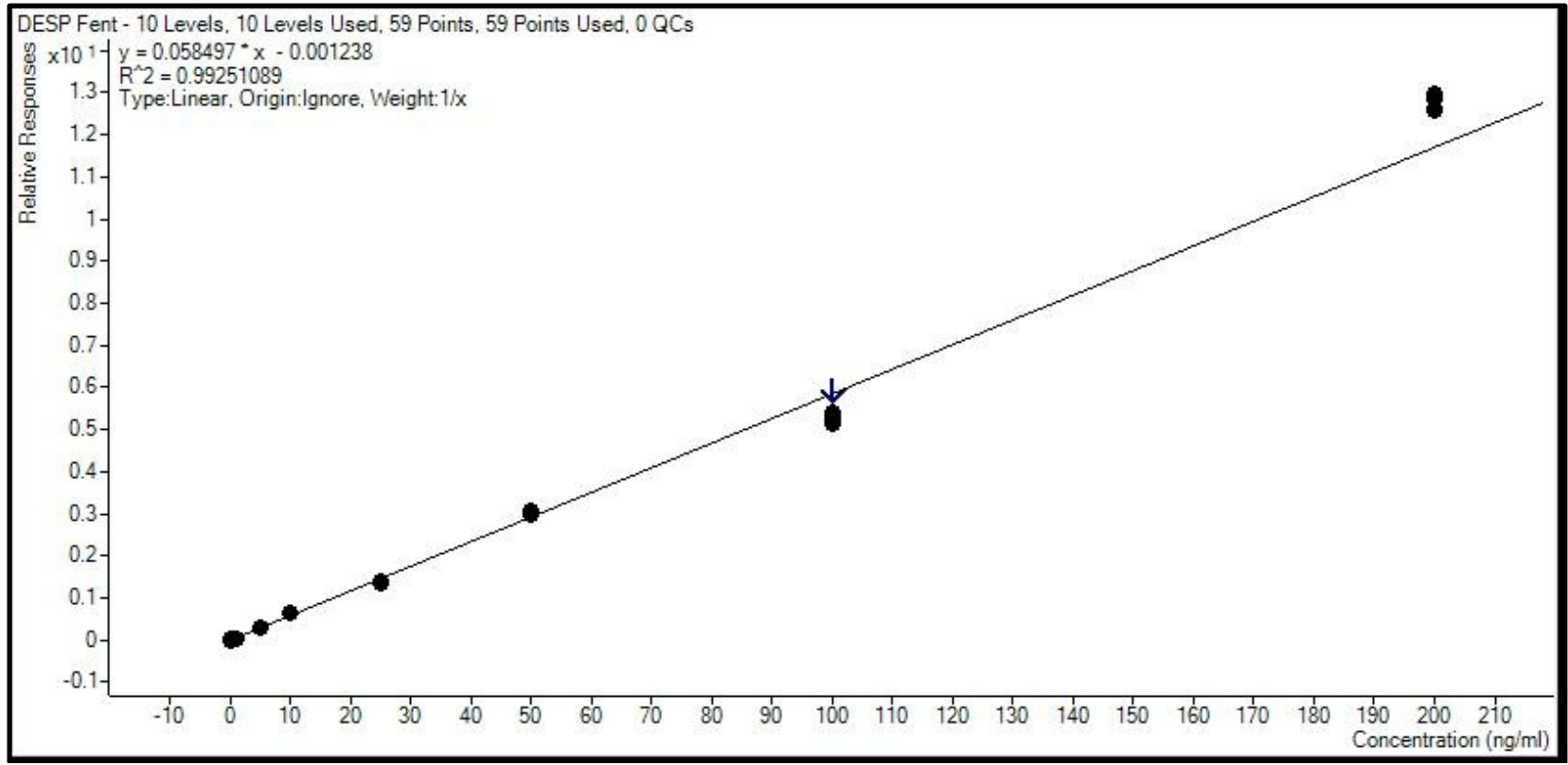




\section{Appendix G - Preliminary Method Development for Methadone}

\section{Methadone}

Methadone (MetD) is a synthetic narcotic and Schedule II drug of abuse. ${ }^{24}$ It is used for medical purposes (Dolophinel囚) in the detoxification and as a maintenance treatment for those addicted to opiates. Methadone can be administered as a pill, orally, or by injection, and is also referred to as Amidone, chocolate chip cookies, fizzies, Maria, Pastora, and wafer.

Dependence is a common health effect for those who abuse the drug.

Withdrawal symptoms include anxiety, tremors, and vomiting. Overdose can lead to repressed respiration, convulsions, coma, and death. ${ }^{24}$ 


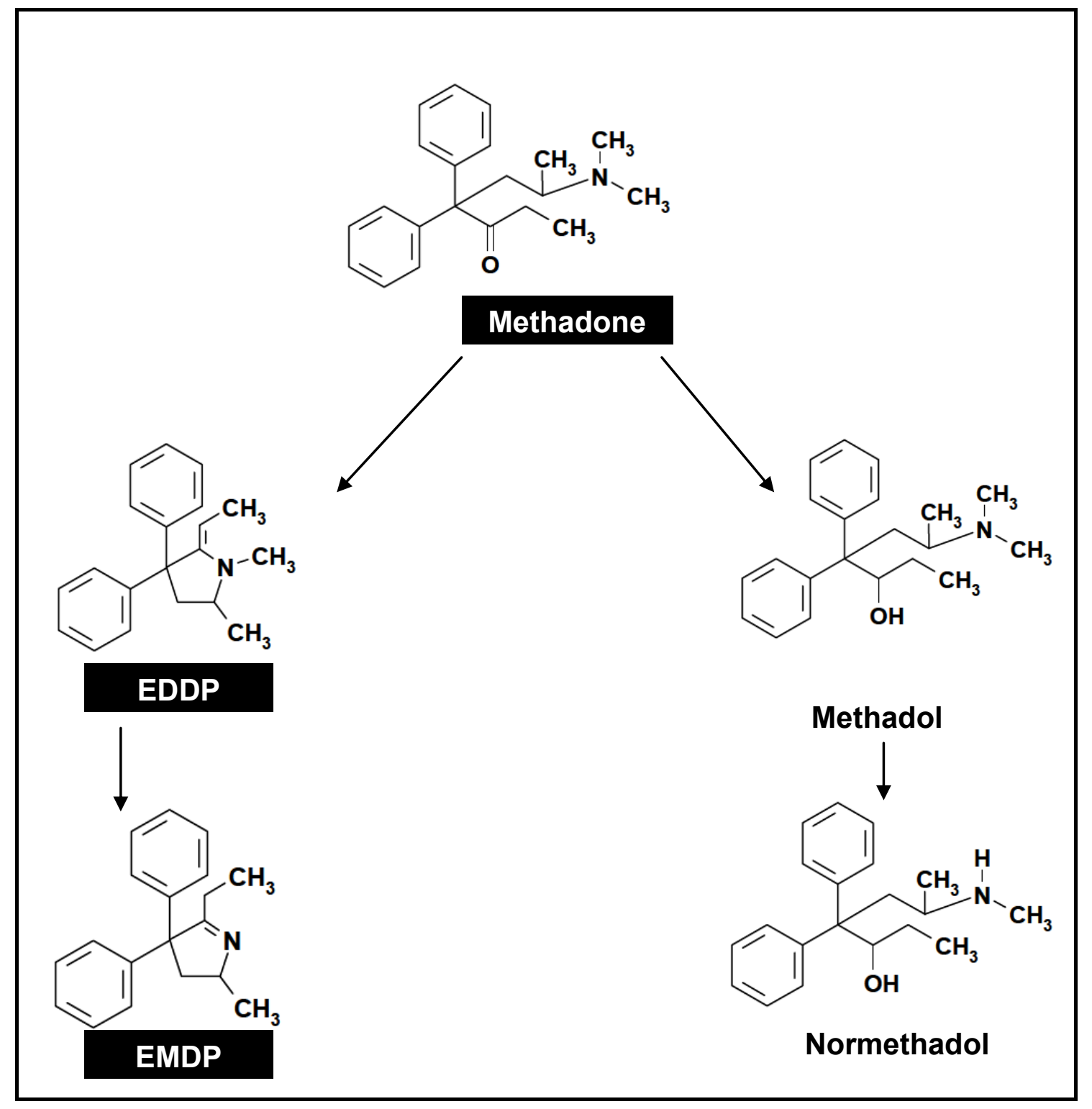

This research focused on specific metabolites 2-Ethylidene-1,5-Dimethyl-3,3Diphenylpyrrolidine (EDDP) and 2-Ethyl-5-Methyl-3,3-Diphenylpyroline (EMDP). Methadone-d3 (MetD-d3) and 2-Ethylidene-1,5-Dimethyl-3,3-Diphenylpyrrolidine-d3 (EDDP-d3) were used as the internal standards. 
Standards were obtained from Cerilliant as $1 \mathrm{mg} / \mathrm{mL}$ for MetD, EDDP, and EMDP and as $100 \mu \mathrm{g} / \mathrm{mL}$ concentration for MetD-d3 and EDDP-d3. The following table lists the MRM transitions for methadone and metabolites found in the reference literature.

\section{Methadone - Compounds, Metabolites, Precursor/Product lons}

Agilent's MassHunter ${ }^{\mathrm{TM}}$ Optimizer Software was used to identify the optimal quantifier and qualifier MRM transitions for methadone and its metabolites under the dissertation research experimental conditions. These values can be found in the following Table.

\section{Methadone Optimized Precursor \& Product lons}

\begin{tabular}{|ccccc|}
\hline \multirow{2}{*}{ Compound } & Fragmentor & CE $(\mathbf{V})$ & Precursor $(\boldsymbol{m} / \mathbf{z})$ & Quantifier $(\mathbf{m} / \mathbf{z})$ \\
\hline MetD-d3 & 100 & 15 & 313.2 & 268.1 \\
& 100 & 27 & 313.2 & 105.0 \\
\hline \multirow{3}{*}{ MetD } & 100 & 15 & 310.2 & 265.0 \\
& 100 & 27 & 310.2 & 104.9 \\
& 100 & 50 & 310.2 & 77.0 \\
& 100 & 19 & 310.2 & 57.0 \\
\hline \multirow{4}{*}{ EDDP } & 160 & 31 & 278.2 & 234.0 \\
& 160 & 19 & 278.2 & 249.1 \\
& 160 & 35 & 278.2 & 186.0 \\
EMDP & 160 & 43 & 278.2 & 219.0 \\
& 130 & 19 & 264.2 & \\
\hline & 130 & 50 & 264.2 & 115.0 \\
& 130 & 43 & 264.2 & 142.5 \\
\hline
\end{tabular}




\section{Appendix H - Preliminary Method Development for Methadone}

\section{Methamphetamine}

Methamphetamine (MetA) is a CNS stimulant and classified by the DEA as a Schedule II drug of abuse. ${ }^{24}$ It is known as Desoxyn® when used for medicinal purposes. Methamphetamine can be snorted, smoked, ingested, or injected. Some of the many street names include bikers, black beauties, chalk, chicken feed, coffee, crank, crystal, glass, go-fast, ice, quick, shards, speed, Tina, trash, and yellow barn. Addiction is common for those who abuse the drug. Effects include anxiety, confusion, paranoia, hallucinations. Methamphetamine use has also been associated with aggressive and violent behavior. Health risks include elevated blood pressure, body temperature, respiration, and heart rate, as well as anorexia, convulsions, dental problems, and death. ${ }^{24}$ 
Metabolic Pathway of Methamphetamine $\mathrm{e}^{51,98-102}$

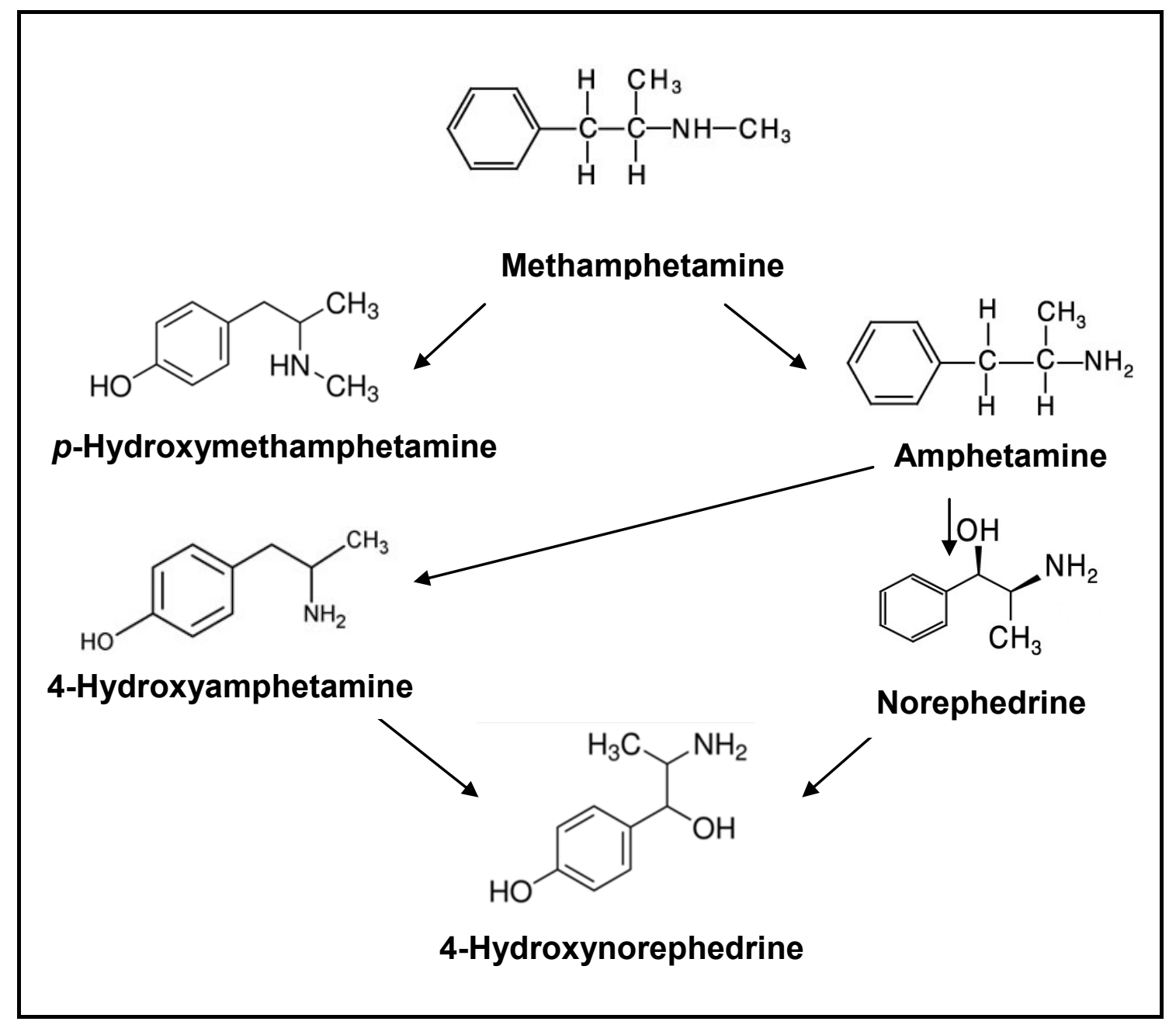

This research focused on specific metabolites $p$-hydroxymethamphetamine $(\mathrm{pHM})$ and amphetamine (AMP). Methamphetamine-D5 (MetA-d5) and amphetamined5 (AMP-d5) were used as internal standards.

Standards were obtained from Cerilliant in the $1 \mathrm{mg} / \mathrm{mL}$ concentration for MetA and AMP and in the $100 \mu \mathrm{g} / \mathrm{mL}$ concentration for MetA-d5 and AMP-d5. The standard for $\mathrm{pHM}$ was obtained from Sigma Aldrich as a $25 \mathrm{mg}$ sample. Agilent's MassHunter ${ }^{\mathrm{TM}}$ Optimizer Software was used to identify the optimal quantifier and qualifier MRM 
transitions for methamphetamine and its metabolites under the dissertation research experimental conditions. These values can be found in the following Table.

Methamphetamine Optimized Precursor \& Product lons

\begin{tabular}{|ccccc|}
\hline \multirow{2}{*}{ Compound } & Fragmentor & CE $(\mathbf{V})$ & Precursor $(\boldsymbol{m} / \mathbf{z})$ & Product $(\boldsymbol{m} / \mathbf{z})$ \\
\hline \multirow{3}{*}{ MetA } & 100 & 19 & 150.1 & 90.9 \\
& 100 & 50 & 150.1 & 65.0 \\
& 100 & 50 & 150.1 & 77.0 \\
& 100 & 23 & 150.1 & 135.0 \\
\hline \multirow{3}{*}{ pHM } & 100 & 19 & 166.1 & 106.9 \\
& 100 & 43 & 166.1 & 77.0 \\
& 100 & 21 & 166.1 & 121.9 \\
& 100 & 23 & 166.1 & 151.0 \\
\hline \multirow{3}{*}{ AMP } & 100 & 19 & 136.1 & 91.0 \\
& 100 & 39 & 136.1 & 65.0 \\
& 100 & 27 & 136.1 & 67.0 \\
& 100 & 15 & 136.1 & 96.8 \\
\hline
\end{tabular}

